\title{
Fermi Liquid Behavior in the 2D Hubbard Model at Low Temperatures
}

\author{
G. Benfatto, A. Giuliani and V. Mastropietro
}

\begin{abstract}
We prove that the weak coupling 2D Hubbard model away from half filling is a Landau Fermi liquid up to exponentially small temperatures. In particular we show that the wave function renormalization is an order 1 constant and essentially temperature independent in the considered range of temperatures and that the interacting Fermi surface is a regular convex curve. This result is obtained by deriving a convergent expansion (which is not a power series) for the two point Schwinger function by Renormalization Group methods and proving at each order suitable power counting improvements due to the convexity of the interacting Fermi surface. Convergence follows from determinant bounds for the fermionic expectations.
\end{abstract}

\section{Introduction and Main result}

\subsection{Motivations}

The Hubbard model, according to many (see for instance [L]), has the same role in the problem of electron correlations as the Ising model in the problem of spin-spin correlations, that is it is the simplest possible model displaying many "real world" features. Still, we are far from a rigorous understanding of many of its properties, except in $1 \mathrm{D}$, where Bethe ansatz arguments $[\mathrm{LW}, \mathrm{G}]$ and Renormalization Group $(\mathrm{RG})$ analysis $[\mathrm{S}, \mathrm{M}]$ provide a quite good understanding of many of its properties up to zero temperature. In more than one dimension only few of its properties are rigorously known: among them we quote the theorems in [L1], establishing the value of the total spin of the ground state in arbitrary dimension (for all densities in the attractive case and at half-filling in the repulsive case), and the bounds in $[\mathrm{KT}]$ on the correlation functions in $2 \mathrm{D}$, ruling out the possibility of magnetic ordering or superconductivity at non zero temperatures. Recent years have seen a great deal of theoretical investigation on the 2D Hubbard model, as such model has been proposed as a basic model for the electronic degrees of freedom of high $T_{c}$ superconductors $[\mathrm{A}]$. Its zero temperature behavior is still rather controversial and 
only approximate analysis have been performed [Me]. On the other hand there are several non trivial and physically important questions for which a rigorous answer is not known, the most important of which is about the Fermi liquid or non Fermi liquid behavior of the 2D Hubbard model; while we refer to the comments after Theorem 1.1 for the precise definition of Fermi liquid, we mention that such question can be answered only by a rather detailed (essentially optimal) knowledge of the temperature dependence of the Schwinger function (hence the bounds in $[\mathrm{KT}]$ cannot be of any help in such a question). There are convincing physical arguments $[\mathrm{VR}]$ supporting the idea that Fermi liquid behavior is crucially related to the convexity of the Fermi surface; in particular the 2D Hubbard model is believed to be a Fermi liquid for densities sufficiently smaller than the half-filling density, i.e., for values of the chemical potential corresponding to a closed and convex free Fermi surface. It is on the other hand well known that the interaction produces a deformation of the Fermi surface $[\mathrm{HM}]$ and it is not guaranteed a priori that the interacting Fermi surface, if existing at all, is still convex. Moreover doubts on the reliability of lowest order approximations, due to a possible non convergence of the series expansion, has been raised recently in the physical literature, see $[\mathrm{A}]$. Therefore, rigorous confirmation on such expectations is much to be desired and we provide it here.

Our main result can be informally stated in the following way (see Theorem 1.1 for a formal statement, the comment after the theorem for the definition of Fermi liquid and (1.7) for the definition of Fermi surface at finite temperature).

For values of the chemical potential smaller than and not too close to $\mu=2$ (the half filled band case) the weak coupling 2D Hubbard model is a Fermi liquid up to exponentially small temperatures, independently on the sign of the interaction. In particular the wave function renormalization is essentially temperature independent and the interacting Fermi surface is a regular convex surface.

In $[R, A M R]$ it has been proved that in the half-filled band case the weak coupling 2D Hubbard model is not a Fermi liquid for temperatures larger than an exponentially small one. Then, combining our results with those in $[R]$, we find that the 2D Hubbard model, for temperatures larger than an exponentially small one, shows a crossover between a Fermi and a non Fermi liquid behavior, while varying the chemical potential from 0 to 2 .

Our main result is proved by showing convergence of a suitable resummation of the weak coupling expansion for the interacting two-point correlation function, needed to take care of the modification of the Fermi surface due to the interaction. In fact the deformation of the Fermi surface has the effect that the naive power series expansion in terms of the free propagator is not convergent at low temperatures. We have then to employ RG methods so that the interacting propagator is dynamically changed ("dressed") at each step of the multiscale analysis; correspondingly the location of the singularity of the interacting propagator will define an interacting Fermi surface which is dynamically modified at each RG step. Convergence of the resummed series follows from power counting improvements at all 
orders due to the convexity of the interacting Fermi surface and from determinant bounds for the fermionic expectations.

\subsection{The Hubbard model}

The Hamiltonian of the 2D Hubbard model is given by

$$
H=\sum_{\vec{x} \in \Lambda} \sum_{\sigma=\uparrow \downarrow} a_{\vec{x}, \sigma}^{+}\left(-\frac{\Delta}{2}-\mu\right) a_{\vec{x}, \sigma}^{-}+U \sum_{\vec{x} \in \Lambda} a_{x, \uparrow}^{+} a_{\vec{x}, \uparrow}^{-} a_{\vec{x}, \downarrow}^{+} a_{\vec{x}, \downarrow}^{-}
$$

where:

1). $\Lambda \subset \mathbb{Z}^{2}$ is a square sublattice of $\mathbb{Z}^{2}$ with side $L$ (the sites will be labelled by $\left.\left(n_{1}, n_{2}\right) \in \mathbb{Z}^{2},-[L / 2] \leq n_{1}, n_{2} \leq[(L-1) / 2]\right)$

2). $a_{\vec{x}, \sigma}^{ \pm}$are creation or annihilation fermionic operators with spin index $\sigma=\uparrow \downarrow$ and site index $\vec{x} \in \Lambda$, satisfying periodic boundary conditions in $\vec{x}$;

3). $\Delta$ is the discrete Laplacean, acting on a function $f: \mathbb{Z}^{2} \rightarrow \mathbb{R}$, periodic of period $L$ in both directions, as: $\Delta f(\vec{x})=\sum_{j=1,2} f\left(\vec{x}+\hat{e}_{j}\right)-2 f(\vec{x})+f\left(\vec{x}-\hat{e}_{j}\right)$, where $\hat{e}_{j}, j=1,2$, are the two unit versors on $\mathbb{Z}^{2}$;

4). $\mu>0$ is the chemical potential, whose value fixes the average density of particles;

5). $U$ is the strength of the on-site density-density interaction; it can be either positive or negative.

We shall also consider the operators $a_{\mathbf{x}, \sigma}^{ \pm}=e^{H x_{0}} a_{\vec{x}, \sigma}^{ \pm} e^{-H x_{0}}$ with $\mathbf{x}=\left(x_{0}, \vec{x}\right)$ and $x_{0} \in[0, \beta]$, for some $\beta>0$; we shall call $x_{0}$ the time variable. The finite temperature Schwinger functions are defined as

$$
S\left(\mathbf{x}_{1}, \sigma_{1}, \varepsilon_{1} ; \ldots ; \mathbf{x}_{n}, \sigma_{n}, \varepsilon_{n}\right)=\frac{\operatorname{Tr} e^{-\beta H} \mathbf{T}\left(a_{\mathbf{x}_{1}, \sigma_{1}}^{\varepsilon_{1}} \cdots a_{\mathbf{x}_{n}, \sigma_{n}}^{\varepsilon_{n}}\right)}{\operatorname{Tr} e^{-\beta H}}
$$

where $\mathbf{x}_{i} \in[0, \beta] \times \Lambda, \sigma_{i}=\uparrow \downarrow, \varepsilon_{i}= \pm$ and $\mathbf{T}$ is the operator of time ordering, acting on a product of fermionic fields as:

$$
\mathbf{T}\left(a_{\mathbf{x}_{1}, \sigma_{1}}^{\varepsilon_{1}} \cdots a_{\mathbf{x}_{n}, \sigma_{n}}^{\varepsilon_{n}}\right)=(-1)^{\pi} a_{\mathbf{x}_{\pi(1)}, \sigma_{\pi(1)}}^{\varepsilon_{\pi(1)}} \cdots a_{\mathbf{x}_{\pi(n)}, \sigma_{\pi(n)}}^{\varepsilon_{\pi(n)}}
$$

where $\pi$ is a permutation of $\{1, \ldots, n\}$, chosen in such a way that $x_{\pi(1) 0} \geq \cdots \geq$ $x_{\pi(n) 0}$, and $(-1)^{\pi}$ is its $\operatorname{sign}^{1}$.

In the non-interacting case $U=0$ the Schwinger functions of any order $n$ can be exactly computed as linear combinations of products of two-point Schwinger functions (via the well-known Wick rule). The two-point Schwinger function itself (also called the free propagator) is equal to:

$$
\left.S_{0}(\mathbf{x}-\mathbf{y}) \equiv S(\mathbf{x}, \sigma,-; \mathbf{y}, \sigma,+)\right|_{U=0}=\frac{1}{\beta L^{2}} \sum_{\mathbf{k} \in \mathcal{D}_{\beta, L}} \frac{e^{-i \mathbf{k} \cdot(\mathbf{x}-\mathbf{y})}}{-i k_{0}+\varepsilon_{0}(\vec{k})-\mu}
$$

\footnotetext{
${ }^{1}$ If some of the time coordinates are equal each other, the arbitrariness of the definition is solved by ordering each set of operators with the same time coordinate so that creation operators precede the annihilation operators.
} 
where:

(a). $\mathbf{k}=\left(k_{0}, \vec{k}\right)$ and $\mathcal{D}_{\beta, L}=\mathcal{D}_{\beta} \times \mathcal{D}_{L}$;

(b). $\mathcal{D}_{\beta}=\left\{k_{0}=\frac{2 \pi}{\beta}\left(n_{0}+\frac{1}{2}\right): n_{0} \in \mathbb{Z}\right\}$ and $\mathcal{D}_{L}=\left\{\vec{k}=\frac{2 \pi}{L}\left(n_{1}, n_{2}\right):-[L / 2] \leq\right.$ $\left.n_{1}, n_{2} \leq[(L-1) / 2]\right\}$

(c). $\varepsilon_{0}(\vec{k})=2-\cos k_{1}-\cos k_{2}$ is the dispersion relation.

Note that $S_{0}(\mathbf{x})$ is a function of $x_{0} \in \mathbb{R}$ antiperiodic of period $\beta$ and that its Fourier transform $\hat{S}_{0}(\mathbf{k})$ is well defined for any $\mathbf{k} \in \mathcal{D}_{\beta, L}$, even in the thermodynamic limit $L \rightarrow \infty$, since $\left|k_{0}\right| \geq \frac{\pi}{\beta}$. We shall refer to this last property by saying that the inverse temperature $\beta$ acts as an infrared cutoff for our theory.

In the limit $\beta, L \rightarrow \infty$ the propagator $\hat{S}_{0}(\mathbf{k})$ becomes singular on the surface $\left\{k_{0}=0\right\} \times \Sigma_{F}^{(0)}$, where $\Sigma_{F}^{(0)} \equiv\left\{\vec{k} \in[-\pi,+\pi] \times[-\pi,+\pi]: \varepsilon_{0}(\vec{k})-\mu=0\right\}$ is the free Fermi surface. It is easy to realize that, if $0<\mu<2$, then $\Sigma_{F}^{(0)}$ is a smooth convex closed curve, symmetric around the point $\vec{k}=(0,0)$. It can be parameterized as $\vec{k}=\vec{p}_{F}^{(0)}(\theta)$ in terms of the polar angle $\theta \in[0,2 \pi]$. We shall also denote $\left|\vec{p}_{F}^{(0)}(\theta)\right|$ by $u^{(0)}(\theta)$.

In order to make apparent the structure of the pole singularity of $\hat{S}_{0}(\mathbf{k})$ at $\{0\} \times \Sigma_{F}^{(0)}$, it is sometimes convenient to rewrite $\hat{S}_{0}(\mathbf{k})$ in the form:

$$
\hat{S}_{0}(\mathbf{k})=\frac{1}{Z_{0}} \frac{1}{-i k_{0}+\vec{v}_{F}^{(0)}(\theta) \cdot\left(\vec{k}-\vec{p}_{F}^{(0)}(\theta)\right)+R(\vec{k})}
$$

where $\theta$ is the polar angle of $\vec{k}, Z_{0}=1$ is the free wave function renormalization and $\vec{v}_{F}^{(0)}(\theta)=\left.\left(\partial \varepsilon_{0} / \partial \vec{k}\right)\right|_{\vec{k}=\vec{p}_{F}(\theta)}$ is the free Fermi velocity. Moreover, near the Fermi surface, $|R(\vec{k})| \leq C\left|\vec{k}-\vec{p}_{F}^{(0)}(\theta)\right|^{2}$, for some positive constant $C$.

\subsection{Main results}

The interacting two-point function $S(\mathbf{x}-\mathbf{y})=S(\mathbf{x}, \sigma,-; \mathbf{y}, \sigma,+)$ turns out to have, in the $L=\infty$ limit, the following structure. Let us call $\hat{S}(\mathbf{k})$ the Fourier transform of $S(\mathbf{x})$ and $\Sigma(\mathbf{k})$ the self-energy, defined as usual by the identity

$$
\hat{S}(\mathbf{k})=\frac{1}{-i k_{0}+\varepsilon_{0}(\vec{k})-\mu+\Sigma(\mathbf{k})} .
$$

By simple symmetry arguments, one can see that $\Sigma\left(k_{0}, \vec{k}\right)=\Sigma\left(k_{0},-\vec{k}\right)=$ $\Sigma^{*}\left(-k_{0}, \vec{k}\right)$; this allows us to introduce the following definitions.

a). The interacting Fermi surface $\Sigma_{F}$ is defined as

$$
\Sigma_{F}=\left\{\vec{k}: \varepsilon_{0}(\vec{k})+\frac{1}{2} \sum_{j= \pm} \Sigma\left(j \pi \beta^{-1}, \vec{k}\right)=\mu\right\},
$$

see also Remark (3) in Section 1.4. We shall be able to parameterize $\Sigma_{F}$ as $\vec{k}=\vec{p}_{F}(\theta)$ in terms of the polar angle $\theta \in[0,2 \pi]$ and we shall denote $\left|\vec{p}_{F}(\theta)\right|$ by $u(\theta)$. 
b). The wave function renormalization is the real quantity

$$
Z(\theta)=1+i \partial_{k_{0}} \Sigma
$$

where $\partial_{k_{0}} \Sigma=(\beta / 2 \pi)\left[\Sigma\left(\frac{\pi}{\beta}, \vec{p}_{F}(\theta)\right)-\Sigma\left(-\frac{\pi}{\beta}, \vec{p}_{F}(\theta)\right)\right]$.

c). The Fermi velocity is the real vector

$$
\vec{v}_{F}(\theta)=\left.\frac{1}{Z(\theta)} \frac{\partial\left(\varepsilon_{0}+\frac{1}{2} \sum_{j= \pm} \Sigma\left(j \pi \beta^{-1}, \vec{k}\right)\right)}{\partial \vec{k}}\right|_{\vec{k}=\vec{p}_{F}(\theta)} .
$$

Our main result is the following.

Theorem 1.1. Let us consider the $2 \mathrm{D}$ Hubbard model with $0<\mu<\mu_{0} \equiv \frac{2-\sqrt{2}}{2}$ and $\beta^{-1} \geq e^{-\frac{a}{|U|}}$ where $a>0$ is a suitable constant. There exists a constant $U_{0}>0$ such that, if $|U| \leq U_{0}$, the two point Schwinger function $\hat{S}(\mathbf{k})$ can be written, in the limit $L=\infty$, as

$$
\hat{S}(\mathbf{k})=\frac{1}{Z(\theta)} \frac{1}{-i k_{0}+\vec{v}_{F}(\theta) \cdot\left(\vec{k}-\vec{p}_{F}(\theta)\right)+R(\mathbf{k})}
$$

with $Z(\theta), \vec{v}_{F}(\theta)$ and $\vec{p}_{F}(\theta)$ real and

$$
\begin{aligned}
Z(\theta) & =1+a(\theta) U^{2}+O\left(U^{3}\right) \\
\vec{v}_{F}(\theta) & =\vec{v}_{F}^{(0)}(\theta)+\vec{b}(\theta) U^{2}+O\left(U^{3}\right) \\
\vec{p}_{F}(\theta) & =\vec{p}_{F}^{(0)}(\theta)+\vec{c}(\theta) U+O\left(U^{2}\right)
\end{aligned}
$$

where $a(\theta),|\vec{b}(\theta)|,|\vec{c}(\theta)|$ are bounded above and below by positive $O(1)$ constants in the region $\beta^{-1} \geq e^{-\frac{a}{|U|}}$. Moreover

$$
|R(\mathbf{k})| \leq C\left[\left|\vec{k}-\vec{p}_{F}(\theta)\right|^{2}+k_{0}^{2}+\left|\vec{k}-\vec{p}_{F}(\theta)\right|\left|k_{0}\right|\right]
$$

for some constant $C>0$.

Remark. One corollary of the proof of Theorem 1.1 is that, under the assumptions of the theorem, one can compute the free energy of the Hubbard model in terms of a convergent expansion (not a power series in $U$ ). In particular it turns out that the first non trivial correction to the free energy coincides with the first order of naive power series expansion in $U$; this means that first order perturbation theory is asymptotically correct as $U \rightarrow 0$. A second corollary of the proof of the theorem is that the decay of $S(\mathbf{x})$ in real space can be estimated as

$$
\left|S(\mathbf{x})-S_{0}(\mathbf{x})\right| \leq C_{N}|U| \frac{1}{1+|\mathbf{x}|} \frac{1}{1+\left(\beta^{-1}|\mathbf{x}|\right)^{N}}
$$

for any positive integer $N$. (1.13) tells us that the interacting Schwinger function $S(\mathbf{x})$ has the same large distance behavior as $S_{0}(\mathbf{x})$, as long as $1 \ll|\mathbf{x}| \ll \beta$; for larger distances the decay is suppressed because of the presence of a non zero temperature $\beta^{-1}$.

The above theorem says that the 2D Hubbard model is a Fermi liquid up to exponentially small temperatures and far from the half filled band case, in the 
following sense. Let us compare the representation (1.10) of the interacting twopoint Schwinger function with the free one, given by (1.5). They are apparently similar but the parameters $Z(\theta), \vec{v}_{F}(\theta)$ and $\vec{p}_{F}(\theta)$, differently from $Z_{0}, \vec{v}_{F}^{(0)}(\theta)$ and $\vec{p}_{F}^{(0)}(\theta)$, are functions of the temperature $\beta^{-1}$, for $\beta^{-1} \geq e^{-a /|U|}$. However such dependence can be stronger or weaker and the different sensitivity to a variation of the temperature has important physical consequences. In the case of the $2 \mathrm{D}$ Hubbard model (1.1) with $\mu<\mu_{0}$ we prove that $Z(\theta), \vec{v}_{F}(\theta)$ and $\vec{p}_{F}(\theta)$ are slowly depending on $\beta$ for $\beta^{-1} \geq e^{-a /|U|}$, that is they are essentially constant in $\beta$ above an exponentially small temperature. This means that, in the considered range of parameters, the interacting two-point correlation is essentially identical to the free one, up to a renormalization of the parameters essentially independent on the temperature; in this sense we say that the system shows a Fermi liquid behavior for temperatures larger than an exponentially small one. This notion of Fermi liquid is the natural mathematical interpretation of the notion of Fermi liquid often used in the theoretical physics literature, and it is essentially the same as the one adopted, for instance, in [Sa1, DR].

Of course the property to be a Fermi liquid (in the above sense) is not trivial at all and it is not verified in many cases. For instance, in the 1D Hubbard model, the wave function renormalization $Z$ depends logarithmically on $\beta$, that is $c_{1} U^{2} \log \beta \leq|Z-1| \leq c_{2} U^{2} \log \beta$, with $c_{1}, c_{2}$ two positive constants, for temperatures above an exponentially small temperature; so, with our definition, the 1D Hubbard model is not a Fermi liquid in such range of temperatures. In the $2 \mathrm{D}$ Hubbard model at half-filling (i.e., at $\mu=2$ ) it has been recently proved $[\mathrm{R}, \mathrm{AMR}]$ that, for temperatures above an exponentially small temperature, $c_{1} U^{2} \log ^{2} \beta \leq|Z-1| \leq c_{2} U^{2} \log ^{2} \beta$, so that the system is not a Fermi liquid at half-filling in that range of temperatures. On the contrary, an example of Fermi liquid in the above sense is provided by the continuum approximation of model (1.1) in $d=2$, the so-called jellium model, for which [DR] showed that, in a range of temperatures above an exponentially small temperature, $c_{1} U^{2} \leq|Z-1| \leq c_{2} U^{2}$, and the system is a Fermi liquid. Note that in the jellium model, due to rotation invariance, the interacting and the free Fermi surfaces have exactly the same shape, that is a circle, and the effect of the interaction essentially consists just in changing its radius.

\subsection{Additional remarks}

1) In the interacting case $U \neq 0$, the Schwinger functions are not exactly computable; they can be written as power series in $U$, convergent for $|U| \leq \varepsilon_{\beta}$, for some constant $\varepsilon_{\beta}$. The power expansion in $U$ is constructed in terms of Feynman diagrams, using as free propagator the function $S_{0}(\mathbf{x})$ in (1.4). All the known bounds on the radius of convergence $\varepsilon_{\beta}$ of this power series shrink to zero as $\beta \rightarrow \infty$. Theorem 1.1 has been proved by constructing a suitable resummed version of the naive power series expansion (needed to take care of the modification of the Fermi surface) which we show to be convergent for $|U| \leq a(\log \beta)^{-1}$. As far as we know, 
the bounds provided by Theorem 1.1 are the best available in the literature. Note that if we contented ourselves of proving the theorem for $|U| \leq\left[C \beta^{\alpha}\right]^{-1}$, with $C, \alpha$ two positive constants, the proof below would have been greatly simplified (no resummations and no multiscale infrared analysis would have been necessary) but of course physically the result would have been much poorer.

2) The theorem is proved in the far from half-filling case, that is for $0<\mu<$ $\mu_{0}=\frac{2-\sqrt{2}}{2}$ (i.e., for values of the average density sufficiently smaller than 1 ). The condition of smallness of the chemical potential guarantees certain strong convexity properties of the free Fermi surface we shall need in the proof below; moreover such condition suppresses a class of possibly dangerous contributions to the power series (the so-called "umklapp" processes with $n \leq 4$ quasi-particles, see below for definitions) that we are not able to control at the present time. We expect that our condition $\mu<\mu_{0}$ is technical and it is not unlikely that our proof could be extended to any $\mu<2$, under the smallness condition $|U| \leq U_{0}(\mu)$, with $\lim _{\mu \rightarrow 2^{-}} U_{0}(\mu)=0$. Our result, combined with the result of [R, AMR] discussed above, implies that the 2D Hubbard model shows a transition from Fermi to nonFermi liquid behavior, in the above range of temperatures, depending on the choice of $\mu$. It would be very interesting to explicitly investigate the crossover between the two regimes.

3) At non zero temperature there is an ambiguity in the definition of the Fermi surface and, in order to resolve this ambiguity, we made the specific choice (1.7). It will be clear from the proof below that any "reasonable" definition of the Fermi surface will have the same regularity properties as those of the one in (1.7). The claim in Theorem 1.1 implies of course that the interacting Fermi surface is a regular and convex curve, uniformly in $\beta$ in the allowed range of temperatures.

4) As already mentioned in item 1 ), Theorem 1.1 is proved by an iterative resummation of the original power series expansion for the two-point correlation function and by a renormalization of the free "measure", which takes into account, in particular, the modification of Fermi surface. This allows us to reexpress iteratively the original power series in $U$ as an expansion in an increasing number of parameters (they are indeed functions), called the "effective couplings" and physically describing the effective interaction at different momentum scales, denoted by $\left(\lambda_{1} \equiv U, \lambda_{0}, \lambda_{-1}, \lambda_{-2}, \ldots\right)$; moreover, the coefficients of the new series are themselves depending on $U$ through the renormalized single scale propagators. The new series will be well defined whenever $\bar{U}=\max _{h \leq 1}\left|\lambda_{h}\right|$ will be smaller than $U_{0}$, where $U_{0}$ is a constant independent of the temperature. From the physical point of view, this means that the temperature dependence at all orders in the expansion for $\hat{S}(\mathbf{k})$ is essentially all included in the effective couplings, whose size in turn will depend strongly on the temperature. The possibility of resumming the perturbative expansion in a new series with the above properties was conjectured in [GS], where the authors proved the claim for the second order contributions. We stress that the possibility of resumming the series into a new series admitting this kind of "uniform bounds" is specific of $d=2$ far from half-filling; for instance 
in $d=1$ the coefficient at order $2 n \geq 2$ of the resummed expansion for $Z(\theta)$ behaves like $\left(\bar{U} / U_{0}\right)^{2 n}(\log \beta)^{n}$, instead of $\left(\bar{U} / U_{0}\right)^{2 n}$, even assuming that the effective interactions are bounded. This is not the case in $d=2$ far from half-filling; in this case the breaking of Fermi liquid behavior can be due only to some instability occurring in the effective interactions.

5) Our result should be compared to [DR], in which a proof of Fermi liquid behavior was given for the jellium model. We have taken from such papers two crucial technical ingredients: the idea of using anisotropic sectors (and the relative sector lemma of [FMRT]) for the bounds and the idea of further decomposing some sector into isotropic sectors in order to improve the bounds for the self energy; note however that the technical implementation of such ideas in the proofs is rather different with respect to [DR], mainly for the heavy use of trees for reorganizing the perturbative series and for the fact that we do not need neither a "1PI analysis" to extract our power counting improvements needed to prove the theorem. Moreover, the presence of a non circular Fermi surface causes many new technical problems with respect to the case in $[\mathrm{DR}]$. The more important one is that, while in the Jellium case the interacting Fermi surface is fixed a priori to be a circle as consequence of rotational symmetry, here on the contrary the shape and the regularity or convexity properties of the interacting Fermi surface are completely unknown: in fact there is no a priori evidence of the fact that the interacting Fermi surface is regular and convex uniformly in $\beta$ in the considered range of temperatures. Hence we cannot in our case fix the interacting Fermi surface by properly tuning the chemical potential, as it is done in $[\mathrm{DR}]$; on the contrary, we proceed in a way similar to that used in $[\mathrm{BM}]$, by inserting at each integration step all the quadratic part of the interaction in the free fermionic measure. In this way to each fermionic integration at a certain momentum scale corresponds a different Fermi surface, and one has to check that the geometrical conditions for defining sectors and to apply the sector lemma are verified at each scale.

6) In $[\mathrm{BGM}]$ a statement similar to Theorem 1.1 above was proved. However in [BGM] the interacting Fermi surface was fixed by adding a suitable counterterm to the free Hamiltonian. The result of Theorem 1.1 cannot be recovered simply by the results of $[\mathrm{BGM}]$, because the inversion of the counterterm must be discussed. This is an highly non trivial problem, that is essentially solved below, by checking that the modified Fermi surface satisfies the same convexity properties of the free one, at each RG step (the choice of dynamically changing the Fermi surface instead of inverting the counterterm is not substantial and the two approaches are essentially equivalent). The inversion problem was discussed at level of perturbation theory in the series of papers [FST] and in [Sa2]. In [PS] it was announced that the inversion problem was solved even at a non perturbative level; a proof is however not yet published.

7) If the temperature is low enough, it is expected that Fermi liquid behavior breaks down, as a consequence of quantum instabilities present in the systems. It is possible to destroy such instabilities by choosing properly an highly non symmetric dispersion relation, for instance by introducing an external magnetic 
field; indeed for such a system in [FKT] a proof of Fermi liquid behavior was given up to zero temperature in $2 \mathrm{D}$. We stress that also in $[\mathrm{FKT}]$ the interacting Fermi surface is not fixed by the symmetries and a counterterm is introduced to fix the interacting Fermi surface; however, the "inversion problem", which we solve for the Hubbard problem in the present paper, is still an open problem in that case. 8) Our results hold for temperatures $T \geq e^{-\frac{a}{|U|}}$ where $a>0$ is a suitable constant depending on the parameters of the free Fermi surface and on the size of the first and second order contributions of the perturbative RG expansion. This kind of result is obtained thanks to the fact that we use an expansion in terms of "effective couplings" in which also the marginal terms, quartic in the fermionic fields, are renormalized. Indeed if such terms were not renormalized one could obtain at most a bound $T \geq e^{-\frac{c_{0}}{1 U}}$, the constant $c_{0}$ being related to an all order bound, like in $[\mathrm{DR}]$; of course it is expected $c_{0} \gg a$. Apart from improving the range of temperatures in which our results hold, the renormalization of the quartic terms is technically very useful, as it greatly simplifies the discussion of the power counting improvements on which the proof is based.

\section{Grassmann functional integrals and Renormalization Group analysis}

\subsection{Grassmann representation}

It is well known that the usual formal power series in $U$ for the partition function and for the Schwinger functions of model (1.1) can be equivalently rewritten in terms of Grassmann functional integrals, defined as follows.

Let us temporarily introduce an ultraviolet cutoff on $k_{0}$ (our lattice model has an intrinsic ultraviolet cut-off in the $\vec{k}$ variables) in the following way. Consider the smooth support function $H_{0}(t), t \in \mathbb{R}$, such that

$$
H_{0}(t)=H_{0}(-t) \begin{cases}=1 & \text { if }|t|<e_{0} / \gamma \\ \in(0,1) & \text { if } e_{0} / \gamma<|t|<e_{0} \\ =0 & \text { if }|t|>e_{0}\end{cases}
$$

with $\gamma=4$ and $e_{0}$ a parameter to be fixed below. Then, given a (large) integer $N$, we restrict the set $\mathcal{D}_{\beta}$ of $k_{0}$ possible values to the set $\left\{k_{0}=\frac{2 \pi}{\beta}\left(n_{0}+\frac{1}{2}\right)\right.$ : $\left.H_{0}\left(\gamma^{-N} k_{0}\right)>0\right\}$, which we shall denote with the same symbol, as well as the set $\mathcal{D}_{\beta, L}$, which is a finite set for each $N$. Given $N$, we consider the Grassmann algebra generated by the Grassmannian variables $\left\{\hat{\psi}_{\mathbf{k}, \sigma}^{ \pm}\right\}_{\mathbf{k} \in \mathcal{D}_{\beta, L}}^{\sigma=\uparrow \downarrow}$ and a Grassmann integration $\int\left[\prod_{\mathbf{k} \in \mathcal{D}_{\beta, L}}^{\sigma=\uparrow \downarrow} d \hat{\psi}_{\mathbf{k}, \sigma}^{+} d \hat{\psi}_{\mathbf{k}, \sigma}^{-}\right]$defined as the linear operator on the Grassmann algebra such that, given a monomial $Q\left(\hat{\psi}^{-}, \hat{\psi}^{+}\right)$in the variables $\hat{\psi}_{\mathbf{k}, \sigma}^{ \pm}$, its action on $Q\left(\hat{\psi}^{-}, \hat{\psi}^{+}\right)$is 0 except in the case $Q\left(\hat{\psi}^{-}, \hat{\psi}^{+}\right)=\prod_{\mathbf{k} \in \mathcal{D}_{\beta, L}}^{\sigma=\uparrow \downarrow} \hat{\psi}_{\mathbf{k}, \sigma}^{-} \hat{\psi}_{\mathbf{k}, \sigma}^{+}$, up to a permutation of the variables. In this case the value of the integral is determined, 
by using the anticommuting properties of the variables, by the condition

$$
\int\left[\prod_{\mathbf{k} \in \mathcal{D}_{\beta, L}}^{\sigma=\uparrow \downarrow} d \hat{\psi}_{\mathbf{k}, \sigma}^{+} d \hat{\psi}_{\mathbf{k}, \sigma}^{-}\right] \prod_{\mathbf{k} \in \mathcal{D}_{\beta, L}}^{\sigma=\uparrow \downarrow} \hat{\psi}_{\mathbf{k}, \sigma}^{-} \hat{\psi}_{\mathbf{k}, \sigma}^{+}=1 .
$$

If we define the free propagator $\hat{g}_{\mathbf{k}}$ as $\hat{g}_{\mathbf{k}}=H_{0}\left(\gamma^{-N} k_{0}\right)\left[-i k_{0}+\varepsilon_{0}(\vec{k})-\mu\right]^{-1}$ and the "Gaussian integration" $P(d \psi)$ as

$$
P(d \psi)=\left[\prod_{\mathbf{k} \in \mathcal{D}_{\beta, L}}^{\sigma=\uparrow \downarrow}\left(L^{2} \beta \hat{g}_{\mathbf{k}}\right) d \hat{\psi}_{\mathbf{k}, \sigma}^{+} d \hat{\psi}_{\mathbf{k}, \sigma}^{-}\right] \cdot \exp \left\{-\sum_{\mathbf{k} \in \mathcal{D}_{\beta, L}}^{\sigma=\uparrow \downarrow}\left(L^{2} \beta \hat{g}_{\mathbf{k}}\right)^{-1} \hat{\psi}_{\mathbf{k}, \sigma}^{+} \hat{\psi}_{\mathbf{k}, \sigma}^{-}\right\},
$$

it holds that

so that

$$
\int P(d \psi) \hat{\psi}_{\mathbf{k}_{1}, \sigma_{1}}^{-} \hat{\psi}_{\mathbf{k}_{2}, \sigma_{2}}^{+}=L^{2} \beta \delta_{\sigma_{1}, \sigma_{2}} \delta_{\mathbf{k}_{1}, \mathbf{k}_{2}} \hat{g}_{\mathbf{k}_{1}}
$$

$$
\lim _{N \rightarrow \infty} \frac{1}{L^{2} \beta} \sum_{\mathbf{k} \in \mathcal{D}_{\beta, L}} e^{-i \mathbf{k}(\mathbf{x}-\mathbf{y})} \hat{g}_{\mathbf{k}}=\lim _{N \rightarrow \infty} \int P(d \psi) \psi_{\mathbf{x}}^{-} \psi_{\mathbf{y}}^{+}=S_{0}(\mathbf{x}-\mathbf{y})
$$

where $S_{0}(\mathbf{x}-\mathbf{y})$ was defined in (1.4) and the Grassmann fields $\psi_{\mathbf{x}, \sigma}^{ \pm}$are defined by

$$
\psi_{\mathbf{x}, \sigma}^{ \pm}=\frac{1}{L^{2} \beta} \sum_{\mathbf{k} \in \mathcal{D}_{\beta, L}} e^{ \pm i \mathbf{k x}} \hat{\psi}_{\mathbf{k}, \sigma}^{ \pm} .
$$

Let us now consider the function on the Grassmann algebra

$$
V(\psi)=U \int d \mathbf{x} \psi_{\mathbf{x}, \uparrow}^{+} \psi_{\mathbf{x}, \uparrow}^{-} \psi_{\mathbf{x}, \downarrow}^{+} \psi_{\mathbf{x}, \downarrow}^{-}
$$

where the symbol $\int d \mathbf{x}$ must be interpreted as

$$
\int d \mathbf{x}=\int_{-\beta / 2}^{+\beta / 2} d x_{0} \sum_{\vec{x} \in \Lambda}
$$

and note that the integral $\int P(d \psi) e^{-V(\psi)}$ is well defined for any $U$; it is indeed a polynomial in $U$, of degree depending on $N$ and $L$. Standard arguments (see, for example [NO], where a different cutoff on $k_{0}$ is used) show that, if there exists the limit of $\int P(d \psi) e^{-V(\psi)}$ as $N \rightarrow \infty$, then the normalized partition function can be written as

$$
e^{-L^{2} \beta F_{L, \beta}} \stackrel{\text { def }}{=} \frac{\operatorname{Tr}\left[e^{-\beta H}\right]}{\operatorname{Tr}\left[e^{-\beta H_{0}}\right]}=\lim _{N \rightarrow \infty} \int P(d \psi) e^{-V(\psi)}
$$

where $H_{0}$ is equal to (1.1) with $U=0$.

Similarly, the Schwinger functions defined in (1.2) can be computed as

$$
S\left(\mathbf{x}_{1}, \sigma_{1}, \varepsilon_{1} ; \ldots ; \mathbf{x}_{n}, \sigma_{n}, \varepsilon_{n}\right)=\lim _{N \rightarrow \infty} \frac{\int P(d \psi) e^{-V(\psi)} \psi_{\mathbf{x}_{1}, \sigma_{1}}^{\varepsilon_{1}} \cdots \psi_{\mathbf{x}_{n}, \sigma_{n}}^{\varepsilon_{n_{n}}}}{\int P(d \psi) e^{-V(\psi)}} .
$$

In the following we shall study the functional integrals by introducing suitable expansions where the value of $N$ has no role and we shall indeed be able to control 
such expansions uniformly in $N$, if $U$ is small enough. Our procedure also implies that we can effectively take the limit $N \rightarrow \infty$ everywhere.

Note that both the Gaussian integration $P(d \psi)$ and the interaction $V(\psi)$ are invariant under the action of the following symmetry transformations:

(1). spin exchange: $\psi_{\mathbf{x}, \uparrow}^{\varepsilon} \longleftrightarrow \psi_{\mathbf{x}, \downarrow}^{\varepsilon}$;

(2). global $U(1): \psi_{\mathbf{x}, \sigma}^{\varepsilon} \rightarrow e^{i \varepsilon \alpha_{\sigma}} \psi_{\mathbf{x}, \sigma}^{\varepsilon}$, with $\alpha_{\sigma} \in \mathbb{R}$ independent of $\mathbf{x}$;

(3). global $S O(2):\left(\begin{array}{c}\psi_{\mathbf{x}, \uparrow}^{\varepsilon} \\ \psi_{\mathbf{x}, \downarrow}^{\varepsilon}\end{array}\right) \rightarrow\left(\begin{array}{cc}\cos \theta & \sin \theta \\ -\sin \theta & \cos \theta\end{array}\right)\left(\begin{array}{c}\psi_{\mathbf{x}, \uparrow}^{\varepsilon} \\ \psi_{\mathbf{x}, \downarrow}^{\varepsilon}\end{array}\right)$, with $\theta \in \mathbb{R}$ independent of $\mathbf{x}$;

(4). parity: $\psi_{\left(x_{0}, \vec{x}\right), \sigma}^{ \pm} \rightarrow \psi_{\left(x_{0},-\vec{x}\right), \sigma}^{ \pm}$;

(5). complex conjugation: $\psi_{\left(x_{0}, \vec{x}\right), \sigma}^{ \pm} \rightarrow \psi_{\left(-x_{0}, \vec{x}\right), \sigma}^{ \pm}, c \rightarrow c^{*}$, where $c$ is a generic constant appearing in the formal action.

\subsection{The ultraviolet integration}

It is convenient, for clarity reasons, to start by studying the free energy $F_{L, \beta}$, defined by (2.9). A preliminary step to our infrared analysis is the integration of the ultraviolet degrees of freedom corresponding to the large values of $k_{0}$. We proceed in the following way. We decompose the free propagator $\hat{g}_{\mathbf{k}}$ into a sum of two propagators supported in the regions of $k_{0}$ "large" and "small", respectively. Given the function $H_{0}(t), t \in \mathbb{R}$, defined in $(2.1)$, we define $\chi(\mathbf{k})=H_{0}\left(\sqrt{k_{0}^{2}+\left[\varepsilon_{0}(\vec{k})-\mu\right]^{2}}\right)$ and $f_{1}(\mathbf{k})=1-\chi(\mathbf{k})$, so that we can rewrite $\hat{g}_{\mathbf{k}}$ as:

$$
\hat{g}_{\mathbf{k}}=f_{1}(\mathbf{k}) \hat{g}_{\mathbf{k}}+\chi(\mathbf{k}) \hat{g}_{\mathbf{k}} \stackrel{\text { def }}{=} \hat{g}^{(+1)}(\mathbf{k})+\hat{g}^{(\leq 0)}(\mathbf{k}) .
$$

We now introduce two independent sets of Grassmann fields $\left\{\psi_{\mathbf{k}, \sigma}^{(+1) \pm}\right\}$ and $\left\{\psi_{\mathbf{k}, \sigma}^{(\leq 0) \pm}\right\}, \mathbf{k} \in \mathcal{D}_{\beta, L}, \sigma=\uparrow \downarrow$, and the Gaussian integrations $P\left(d \psi^{(+1)}\right)$ and $P\left(d \psi^{(\leq 0)}\right)$, defined by

$$
\begin{aligned}
& \int P\left(d \psi^{(+1)}\right) \hat{\psi}_{\mathbf{k}_{1}, \sigma_{1}}^{(+1)-} \hat{\psi}_{\mathbf{k}_{2}, \sigma_{2}}^{(+1)+}=L^{2} \beta \delta_{\sigma_{1}, \sigma_{2}} \delta_{\mathbf{k}_{1}, \mathbf{k}_{2}} \hat{g}^{(+1)}\left(\mathbf{k}_{1}\right), \\
& \int P\left(d \psi^{(\leq 0)}\right) \hat{\psi}_{\mathbf{k}_{1}, \sigma_{1}}^{(\leq 0)-} \hat{\psi}_{\mathbf{k}_{2}, \sigma_{2}}^{(\leq 0)+}=L^{2} \beta \delta_{\sigma_{1}, \sigma_{2}} \delta_{\mathbf{k}_{1}, \mathbf{k}_{2}} \hat{g}^{(\leq 0)}\left(\mathbf{k}_{1}\right) .
\end{aligned}
$$

Similarly to $P(d \psi)$, the Gaussian integrations $P\left(d \psi^{(+1)}\right), P\left(d \psi^{(\leq 0)}\right)$ also admit an explicit representation analogous to $(2.3)$, with $\hat{g}_{\mathbf{k}}$ replaced by $\hat{g}^{(+1)}(\mathbf{k})$ or $\hat{g}^{(\leq 0)}(\mathbf{k})$ and the sum over $\mathbf{k}$ restricted to the values in the support of $\chi(\mathbf{k})$ or $f_{1}(\mathbf{k})$, respectively; moreover, for any $N \geq 1, P\left(d \psi^{(\leq 0)}\right)$ is independent of $N$. The definition of Grassmann integration implies the following identity ("addition principle"):

$$
\int P(d \psi) e^{-V(\psi)}=\int P\left(d \psi^{(\leq 0)}\right) \int P\left(d \psi^{(+1)}\right) e^{-V\left(\psi^{(\leq 0)}+\psi^{(+1)}\right)}
$$


so that we can rewrite $F_{L, \beta}$ as

$$
\begin{aligned}
e^{-L^{2} \beta F_{L, \beta}} & =\int P\left(d \psi^{(\leq 0)}\right) \exp \left\{\sum_{n \geq 1} \frac{1}{n !} \mathcal{E}_{1}^{T}\left(-V\left(\psi^{(\leq 0)}+\cdot\right) ; n\right)\right\} \equiv \\
& \equiv e^{-L^{2} \beta F_{0}} \int P\left(d \psi^{(\leq 0)}\right) e^{-\mathcal{V}^{(0)}\left(\psi^{(\leq 0)}\right)}
\end{aligned}
$$

where the truncated expectation $\mathcal{E}_{1}^{T}$ is defined, given any polynomial $V_{1}\left(\psi^{(+1)}\right)$ with coefficient depending on $\psi^{(\leq 0)}$, as

$$
\mathcal{E}_{1}^{T}\left(V_{1}(\cdot) ; n\right)=\left.\frac{\partial^{n}}{\partial \lambda^{n}} \log \int P\left(d \psi^{(+1)}\right) e^{\lambda V_{1}\left(\psi^{(+1)}\right)}\right|_{\lambda=0}
$$

and $\mathcal{V}^{(0)}$, which of course depends on the ultraviolet cutoff, is fixed by the condition $\mathcal{V}^{(0)}(0)=0$. It can be shown (see Appendix A) that $\mathcal{V}^{(0)}$ can be written as

$$
\begin{gathered}
\mathcal{V}^{(0)}\left(\psi^{(\leq 0)}\right)=\sum_{l=1}^{\infty} \sum_{\sigma_{1}, \ldots, \sigma_{l}=\uparrow \downarrow} \int d \mathbf{x}_{1} \cdots d \mathbf{x}_{2 l} . \\
\cdot\left[\prod_{i=1}^{l} \psi_{\mathbf{x}_{2 i-1}, \sigma_{i}}^{(\leq 0)+} \psi_{\mathbf{x}_{2 i}, \sigma_{i}}^{(\leq 0)-}\right] W_{2 l}^{(0)}\left(\mathbf{x}_{1}, \ldots, \mathbf{x}_{2 l}\right)
\end{gathered}
$$

where the integrations $\int d \mathbf{x}_{i}$ must be interpreted as in (2.8). The possibility of representing $\mathcal{V}^{(0)}$ in the form (2.16), with the kernels $W_{2 l}^{(0)}$ independent of the spin indices $\sigma_{i}$, follows from the symmetries listed above, after (2.10). For each fixed $N$, the kernels $W_{2 l}^{(0)}$ vanish for $l$ large enough; moreover they are translation invariant and are given, in the limit $N=+\infty$, by power series in $U$, convergent for $U$ small enough; finally, for any family $\left\{m_{i j}, 1 \leq i<j \leq 2 l\right\}$, of non negative integers such that $m=\sum_{1 \leq i<j \leq 2 l} m_{i j} \leq 2$, there exists $U_{0}$ such that, if $|U| \leq U_{0}$,

$$
\int d \mathbf{x}_{1} \cdots d \mathbf{x}_{2 l}\left[\prod_{1 \leq i<j \leq 2 l}\left|\vec{x}_{i}-\vec{x}_{j}\right|^{m_{i j}}\right]\left|W_{2 l}^{(0)}\left(\mathbf{x}_{1}, \ldots, \mathbf{x}_{2 l}\right)\right| \leq L^{2} \beta C^{l}|U|^{\max \{1, l-1\}}
$$

for some constant $C>0$. The proof of (2.17) is based on a multiscale analysis of the ultraviolet integration over the time coordinates (much simpler than the infrared integration we shall study below) and it is sketched in Appendix A.

\subsection{Renormalization of the free measure}

The Grassmann integral in the r.h.s. of (2.14) is computed iteratively in the following way. We step by step decompose the propagator into a sum of two propagators, the first supported on momenta $\sim \gamma^{h}, h \leq 0$ (here $\gamma$ is the same scaling parameter appearing in (2.1)), the second supported on momenta smaller than $\gamma^{h}$. Correspondingly we rewrite the Grassmann field as a sum of two independent fields: 
$\psi^{(\leq h)}=\psi^{(h)}+\psi^{(\leq h-1)}$ and we integrate the field $\psi^{(h)}$, in analogy with $(2.14)$. In this way we inductively prove that, for any $h \leq 0,(2.14)$ can be rewritten as

$$
e^{-L^{2} \beta F_{L, \beta}}=e^{-L^{2} \beta F_{h}} \int P_{E_{h}, C_{h}}\left(d \psi^{(\leq h)}\right) e^{-\mathcal{V}^{(h)}(\psi \leq h)},
$$

where: $\mathcal{V}^{(h)}$ can be represented by an expansion similar to $(2.16)$, with $\psi^{(\leq 0)}$ replaced by $\psi^{(\leq h)}$ and the kernels $W_{2 l}^{(0)}$ replaced by new kernels $W_{2 l}^{(h)}$; the Grassmann integration $P_{E_{h}, C_{h}}\left(d \psi^{(\leq h)}\right)$ can be represented as

$$
\begin{aligned}
& P_{E_{h}, C_{h}}\left(d \psi^{(\leq h)}\right)=\left[\prod_{\mathbf{k} \in \mathcal{D}_{\beta, L}^{*}}^{\sigma=\uparrow \downarrow}\left(\frac{L^{2} \beta C_{h}^{-1}(\mathbf{k})}{-i k_{0}+E_{h}(\mathbf{k})-\mu}\right) d \psi_{\mathbf{k}, \sigma}^{(\leq h)+} d \psi_{\mathbf{k}, \sigma}^{(\leq h)-}\right] . \\
& \cdot \exp \left\{-\frac{1}{L^{2} \beta} \sum_{\mathbf{k} \in \mathcal{D}_{\beta, L}^{*}}^{\sigma=\uparrow \downarrow} C_{h}(\mathbf{k})\left(-i k_{0}+E_{h}(\mathbf{k})-\mu\right) \hat{\psi}_{\mathbf{k}, \sigma}^{+(\leq h)} \hat{\psi}_{\mathbf{k}, \sigma}^{-(\leq h)}\right\},
\end{aligned}
$$

where $E_{h}(\mathbf{k})$ is a function to be iteratively constructed below, with $E_{0}\left(k_{0}, \vec{k}\right) \equiv$ $\varepsilon_{0}(\vec{k})$. Moreover $C_{h}(\mathbf{k})^{-1}$ is a compact support function defined as

$$
C_{h}^{-1}(\mathbf{k})=H_{0}\left[\gamma^{-h}\left|-i k_{0}+E_{h}(\mathbf{k})-\mu\right|\right]
$$

and $\mathcal{D}_{\beta, L}^{*}$ the restriction of $\mathcal{D}_{\beta, L}$ to the set of momenta in the support of $C_{h}^{-1}(\mathbf{k})$. Note that, for $h=0$, the Gaussian integration $P_{E_{0}, C_{0}}\left(d \psi^{(\leq 0)}\right)$ coincides with the integration $P\left(d \psi^{(\leq 0)}\right)$ defined above. So, by this remark, we see that the representation $(2.18)-(2.19)$ is true at the first step $h=0$. In order to inductively prove it for $h<0$ we proceed as follows. We introduce the localization operator as a linear operator acting on the kernels of $\mathcal{V}^{(h)}$ in the following way:

$$
\mathcal{L} W_{2 l}^{(h)}\left(\mathbf{x}_{1}, \ldots, \mathbf{x}_{2 l}\right)= \begin{cases}W_{2 l}^{(h)}\left(\mathbf{x}_{1}, \ldots, \mathbf{x}_{2 l}\right) & \text { if } l=1,2 \\ 0 & \text { if } l \geq 3 .\end{cases}
$$

We also define $\mathcal{R}$ as $\mathcal{R}=1-\mathcal{L}$ and rewrite the r.h.s. of (2.18) as

$$
e^{-L^{2} \beta F_{h}} \int P_{E_{h}, C_{h}}\left(d \psi^{(\leq h)}\right) e^{-\mathcal{L V}^{(h)}\left(\psi^{(\leq h)}\right)-\mathcal{R} \mathcal{V}^{(h)}\left(\psi^{(\leq h)}\right)},
$$

where by definition $\mathcal{L} \mathcal{V}^{(h)}$ can be written as

$$
\begin{aligned}
& \mathcal{L} \mathcal{V}^{(h)}=\sum_{\sigma=\uparrow \downarrow} \int d \mathbf{x} d \mathbf{y} n_{h}(\mathbf{x}-\mathbf{y}) \psi_{\mathbf{x}, \sigma}^{(\leq h)+} \psi_{\mathbf{y}, \sigma}^{(\leq h)-}+ \\
& +\sum_{\sigma, \sigma^{\prime}=\uparrow \downarrow} \int d \mathbf{x}_{1} \ldots d \mathbf{x}_{4} \lambda_{h}\left(\mathbf{x}_{1} \ldots \mathbf{x}_{4}\right) \psi_{\mathbf{x}_{1}, \sigma}^{(\leq h)+} \psi_{\mathbf{x}_{2}, \sigma}^{(\leq h)-} \psi_{\mathbf{x}_{3}, \sigma^{\prime}}^{(\leq h)+} \psi_{\mathbf{x}_{4}, \sigma^{\prime}}^{(\leq h)-} .
\end{aligned}
$$

Now, calling $\hat{n}_{h}(\mathbf{k})$ the Fourier transform of $n_{h}(\mathbf{x})$ and defining

$$
E_{h-1}(\mathbf{k})=E_{h}(\mathbf{k})+C_{h}^{-1}(\mathbf{k}) \hat{n}_{h}(\mathbf{k}),
$$


we can rewrite $(2.22)$ as

$$
e^{-L^{2} \beta\left(F_{h}+t_{h}\right)} \int P_{E_{h-1}, C_{h}}\left(d \psi^{(\leq h)}\right) e^{-\mathcal{L}_{4} \mathcal{V}^{(h)}\left(\psi^{\leq h}\right)-\mathcal{R} \mathcal{V}^{(h)}\left(\psi^{(\leq h)}\right)},
$$

where $t_{h}$ is a constant which takes into account the change in the renormalization factor of the measure, of size $|U||h| \gamma^{2 h}$, as it follows from the bound in the first line of (2.38) below. Moreover

$$
\mathcal{L}_{4} \mathcal{V}^{(h)}=\sum_{\sigma, \sigma^{\prime}=\uparrow \downarrow} \int d \mathbf{x}_{1} \ldots d \mathbf{x}_{4} \lambda_{h}\left(\mathbf{x}_{1} \ldots \mathbf{x}_{4}\right) \psi_{\mathbf{x}_{1}, \sigma}^{(\leq h)+} \psi_{\mathbf{x}_{2}, \sigma}^{(\leq h)-} \psi_{\mathbf{x}_{3}, \sigma^{\prime}}^{(\leq h)+} \psi_{\mathbf{x}_{4}, \sigma^{\prime}}^{(\leq h)-}
$$

We now define $\hat{\mathcal{V}}^{(h)} \equiv \mathcal{L}_{4} \mathcal{V}^{(h)}+\mathcal{R} \mathcal{V}^{(h)}$ and again use the addition principle in order to rewrite $(2.25)$ as

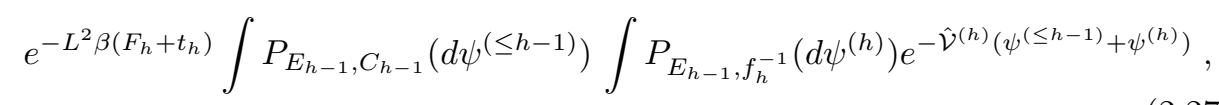

with $P_{E_{h-1}, f_{h}^{-1}}\left(d \psi^{(h)}\right)$ a Grassmann Gaussian integration such that

$$
\begin{aligned}
& \int P_{E_{h-1}, f_{h}^{-1}}\left(d \psi^{(h)}\right) \hat{\psi}_{\mathbf{k}_{1}, \sigma_{1}}^{(h)-} \hat{\psi}_{\mathbf{k}_{2}, \sigma_{2}}^{(h)+}=L^{2} \beta \delta_{\mathbf{k}_{1}, \mathbf{k}_{2}} \delta_{\sigma_{1}, \sigma_{2}} \hat{g}^{(h)}\left(\mathbf{k}_{1}\right), \\
& \quad \text { with } \hat{g}^{(h)}(\mathbf{k})=\frac{f_{h}(\mathbf{k})}{-i k_{0}+E_{h-1}(\mathbf{k})-\mu}
\end{aligned}
$$

and

$$
f_{h}(\mathbf{k})=H_{0}\left[\gamma^{-h}\left|-i k_{0}+E_{h}(\mathbf{k})-\mu\right|\right]-H_{0}\left[\gamma^{-h+1}\left|-i k_{0}+E_{h-1}(\mathbf{k})-\mu\right|\right] .
$$

If we now define

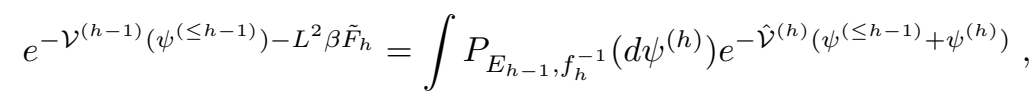

it is easy to see that $\mathcal{V}^{(h-1)}$ is of the form $(2.16)$, with $\psi^{(\leq 0)}$ replaced by $\psi^{(\leq h-1)}$ and the kernels $W_{2 l}^{(0)}$ replaced by new kernels $W_{2 l}^{(h-1)}$, and that

$$
F_{h-1}=F_{h}+t_{h}+\tilde{F}_{h} .
$$

It is sufficient to use the identity

$$
\mathcal{V}^{(h-1)}\left(\psi^{(\leq h-1)}\right)+L^{2} \beta \tilde{F}_{h}=\sum_{n \geq 1} \frac{1}{n !}(-1)^{n} \mathcal{E}_{h}^{T}\left(\hat{\mathcal{V}}^{(h)}\left(\psi^{(\leq h-1)}+\cdot\right) ; n\right)
$$

where $\mathcal{E}_{h}^{T}$ is the truncated expectation with respect to the propagator $\hat{g}^{(h)}(\mathbf{k})$. Moreover the symmetry relations listed after (2.10) are still satisfied, because the symmetry properties of the free integration are not modified by the renormalization procedure, so that the effective potential $\mathcal{V}^{(h)}$ on scale $h$ has the same symmetries as $\mathcal{V}^{(0)}$ and in particular it can be expanded in the form $(2.16)$, with kernels $W_{2 l}^{(h)}$ independent of the spin labels. 
We iterate this procedure up to the first scale $h_{\beta}$ such that

$$
\gamma^{h_{\beta}-1} e_{0}<\min \left\{\left|k_{0}-\operatorname{Im} E_{h_{\beta}}(\mathbf{k})\right|, \mathbf{k} \in \mathcal{D}_{\beta, L}, C_{h_{\beta}}^{-1}(\mathbf{k})>0\right\}
$$

where $e_{0}$ is the same of (2.1). By the properties of $E_{h}(\mathbf{k})$, that will be described and proved below, it will turn out that $h_{\beta}$ is finite and actually larger than $\left[\log _{\gamma}\left(\pi / 2 e_{0} \beta\right)\right]$, see comments after $(2.51)$. Moreover, this definition is such that $f_{h_{\beta}}(\mathbf{k})=C_{h_{\beta}}^{-1}(\mathbf{k})$, hence the propagator associated with $P_{E_{h_{\beta}-1}, C_{h_{\beta}}}\left(d \psi^{\left(\leq h_{\beta}\right)}\right)$ is given by $\hat{g}^{\left(h_{\beta}\right)}(\mathbf{k})$.

On scale $h_{\beta}$ we define

$$
e^{-L^{2} \beta\left(\tilde{F}_{h_{\beta}}+t_{h_{\beta}}\right)}=\int P_{E_{h_{\beta}-1}, C_{h_{\beta}}}\left(d \psi^{\left(\leq h_{\beta}\right)}\right) e^{-\hat{\mathcal{V}}^{\left(h_{\beta}\right)}\left(\psi^{\left(\leq h_{\beta}\right)}\right)},
$$

so that we have

$$
F_{L, \beta}=F_{0}+\sum_{h=h_{\beta}}^{0}\left(\tilde{F}_{h}+t_{h}\right)
$$

Note that the above procedure allows us to rewrite the effective coupling on scale $h$ $\lambda_{h}(\underline{\mathbf{x}}), \underline{\mathbf{x}}=\left\{\mathbf{x}_{1}, \mathbf{x}_{2}, \mathbf{x}_{3}, \mathbf{x}_{4}\right\}$, and the renormalized dispersion relation $E_{h}(\mathbf{k})$ as functionals of $U$ and $\lambda_{j}, E_{j}$, with $h<j \leq 0$ :

$$
\begin{aligned}
E_{h-1}(\mathbf{k}) & =E_{h}(\mathbf{k})+C_{h}^{-1}(\mathbf{k}) \hat{\beta}_{h}^{2}\left(\mathbf{k} ; E_{h}, \lambda_{h}, \ldots, E_{0}, \lambda_{0}, U\right), \\
\lambda_{h-1}(\underline{\mathbf{x}}) & =\lambda_{h}(\underline{\mathbf{x}})+\beta_{h}^{4}\left(\underline{\mathbf{x}} ; E_{h}, \lambda_{h}, \ldots, E_{0}, \lambda_{0}, U\right) .
\end{aligned}
$$

The functionals $\hat{\beta}_{h}^{2}$ and $\beta_{h}^{4}$ are called the $E$-component and the $\lambda$-component of the Beta function.

The key point of the subsequent discussion will be the fact that the kernels of the effective potentials $\mathcal{V}^{(h)}$ will be given by convergent power series in $U, \lambda_{j}$, $h \leq j \leq 0$, under some smallness and smoothness conditions of $E_{h}$ and $\lambda_{j}$. Once identified the conditions on $E_{h}$ and $\lambda_{h}$ sufficient for proving convergence of the series, we shall inductively check such conditions.

The property $\lambda_{h}$ has to satisfy so that the iterative construction is well defined is a smallness property, a bit stronger than the request:

$$
\left|\hat{\lambda}_{h}\left(\mathbf{k}_{1}, \mathbf{k}_{2}, \mathbf{k}_{3}, \mathbf{k}_{1}-\mathbf{k}_{2}+\mathbf{k}_{3}\right)\right| \leq U_{0}
$$

where $\hat{\lambda}_{h}\left(\mathbf{k}_{1}, \mathbf{k}_{2}, \mathbf{k}_{3}, \mathbf{k}_{1}-\mathbf{k}_{2}+\mathbf{k}_{3}\right)$ is the Fourier transform of $\lambda_{h}\left(\mathbf{x}_{1}, \mathbf{x}_{2}, \mathbf{x}_{3}, \mathbf{x}_{4}\right)$ and $U_{0}$ is small enough. The precise statement of the smallness property we need to require on $\lambda_{h}$ is a bit technical, in particular it involves the definitions of "sectors" and "modified running coupling functions" and we postpone it to next subsections, see (2.92) below. 

following:

The smallness and smoothness properties that $E_{h}(\mathbf{k})$ has to satisfy are the

$$
\begin{gathered}
\left|E_{h}(\mathbf{k})-E_{h-1}(\mathbf{k})\right| \leq C_{0}|U||h| \gamma^{2 h} \mathbb{1}\left(C_{h}^{-1}(\mathbf{k})>0\right) \\
\left|\partial_{k_{i_{1}}} \cdots \partial_{k_{i_{n}}}\left(E_{h}(\mathbf{k})-E_{h-1}(\mathbf{k})\right)\right| \leq \\
\quad \leq\left\{\begin{array}{ll}
C_{n}|U||h| \gamma^{(2-n) h} & \text { if } 0<C_{h}^{-1}(\mathbf{k})<1 \\
C_{n}|U|^{2}|h| \gamma^{(2-n) h} & \text { if } C_{h}^{-1}(\mathbf{k})=1
\end{array}, \quad n \geq 1\right.
\end{gathered}
$$

where the function $\mathbb{1}$ (condition) is the function $=1$ if condition is satisfied and $=0$ otherwise, and $i_{j} \in\{0,1,2\}, j=1, \ldots, n$, and $\partial_{k_{i}}$ must be interpreted as discrete derivatives in the three coordinate directions ${ }^{2}$, acting on functions $f: \mathcal{D}_{\beta, L} \rightarrow \mathbb{R}$ as:

$$
\begin{aligned}
& \partial_{k_{0}} f\left(k_{0}, k_{1}, k_{2}\right)=\frac{\beta}{2 \pi}\left[f\left(k_{0}+\frac{2 \pi}{\beta}, k_{1}, k_{2}\right)-f\left(k_{0}, k_{1}, k_{2}\right)\right], \\
& \partial_{k_{1}} f\left(k_{0}, k_{1}, k_{2}\right)=\frac{L}{2 \pi}\left[f\left(k_{0}, k_{1}+\frac{2 \pi}{L}, k_{2}\right)-f\left(k_{0}, k_{1}, k_{2}\right)\right], \\
& \partial_{k_{2}} f\left(k_{0}, k_{1}, k_{2}\right)=\frac{L}{2 \pi}\left[f\left(k_{0}, k_{1}, k_{2}+\frac{2 \pi}{L}\right)-f\left(k_{0}, k_{1}, k_{2}\right)\right] .
\end{aligned}
$$

The reason for having two different bounds depending on the value of $C^{-1}(\mathbf{k})$ in the second of (2.38) depends on the very definition of $E_{h-1}(\mathbf{k})-E_{h}(\mathbf{k})$ : by $(2.24)$ such difference is equal to $C^{-1}(\mathbf{k}) \hat{n}_{h}(\mathbf{k})$ with $\hat{n}_{h}(\mathbf{k})$ a function that will be proved to be bounded proportionally to $|U||h| \gamma^{2 h}$ and with the $n$-th derivative bounded proportionally to $|U|^{2}|h| \gamma^{(2-n) h}$; when deriving $E_{h-1}(\mathbf{k})-E_{h}(\mathbf{k})$ the derivatives can all fall onto $C^{-1}(\mathbf{k})$, in which case we get a term proportional to $|U||h| \gamma^{(2-n) h}$ (non vanishing only in the region were $C^{-1}(\mathbf{k})$ is non constant).

Note also that, by the parity and complex conjugation symmetries listed after $(2.10), E_{h}(\mathbf{k})$ satisfies the symmetry properties

$$
E_{h}\left(k_{0}, \vec{k}\right)=E_{h}\left(k_{0},-\vec{k}\right)=E_{h}^{*}\left(-k_{0}, \vec{k}\right) .
$$

Note that at the first step $h=0$ the symmetry (2.40) is true, by the explicit form of $\varepsilon_{0}(\vec{k})$ and the definition of $E_{0}(\mathbf{k})$.

\footnotetext{
${ }^{2}$ In the following we will be interested in studying the $L \rightarrow \infty$ limit of the free energy and two-point correlation function. For this reason, even if, strictly speaking, the rigorous way to proceed should be performing bounds at finite $L$, showing uniformity in $L$ and then performing the limit, in order not to make the notation too cumbersome and not to hide the ideas behind the proof, we shall sometimes proceed formally by replacing the discrete derivatives in spatial direction by their formal $L \rightarrow \infty$ limit, and the sums $(2 \pi / L)^{2} \sum_{\vec{k} \in \mathcal{D}_{L}}$ by $\int_{-\pi}^{\pi} \int_{-\pi}^{\pi} d \vec{k}$. Being the bounds we perform purely dimensional, it can be realized that our bounds can be adapted also to the finite $L$ case, and the resulting estimates turn out to be uniform in $L$ for $L$ big enough. A rigorous discussion of the uniformity of the bounds at finite $L$ for $L$ big enough in a case similar to the one discussed in this paper can be found in $[\mathrm{BM}]$.
} 
We now proceed as follows. In the following subsections 2.4-2.9 we shall: describe the geometric properties of the Fermi surface on scale $h$, which will be crucial for the subsequent inductive bounds; describe the perturbative expansion in $\left(\lambda_{h}, \ldots, \lambda_{0}, U\right)$ for the effective potential $\mathcal{V}^{(h)}$; resume the inductive procedure described in [BGM] (adapted to the present case) allowing to prove that, under the above smallness and smoothness conditions on $E_{h}$ and $\lambda_{h}$, the expansion for $\mathcal{V}^{(h)}$ is well defined; adapt the expansion for the free energy to the computation of the two-point Schwinger function and complete the proof of the Main Theorem, under the above smallness and smoothness conditions on $E_{h}$ and $\lambda_{h}$. Then in Section 3 we shall inductively prove the smallness and smoothness properties of $E_{h}$ and $\lambda_{h}$. While the smallness condition on $\lambda_{h}$ will be controlled as in [BGM], by imposing a condition on the temperature, the inductive proof of the smallness and smoothness conditions on $E_{h}$ is the main novelty (and the real new technical difficulty) with respect to $[\mathrm{BGM}]$. This will conclude the proof of Main Theorem in the introduction.

\subsection{The Fermi surface at scale $h$}

Given $h_{\beta} \leq h \leq 0$ and $E_{h}(\mathbf{k})$, we define an effective dispersion relation on scale $h$ as

$$
\varepsilon_{h}(\vec{k}) \stackrel{\text { def }}{=} \frac{1}{2}\left[E_{h}\left(\frac{\pi}{\beta}, \vec{k}\right)+E_{h}\left(-\frac{\pi}{\beta}, \vec{k}\right)\right] .
$$

Remark. Note that, thanks to $(2.40), \varepsilon_{h}(\vec{k})$ is real and $\varepsilon_{h}(\vec{k})=\varepsilon_{h}(-\vec{k})$.

A crucial consequence of the properties (2.38) and of the explicit form of the unperturbed dispersion relation $\varepsilon_{0}(\vec{k})$ is that at any scale $h \leq 0$ we can define a Fermi surface $\Sigma_{F}^{(h)}=\left\{\vec{k}: \varepsilon_{h}(\vec{k})-\mu=0\right\}$ with strong convexity properties.

Let us start by stating the properties of the free dispersion relation $\varepsilon_{0}(\vec{k})$ we shall use in the following.

1. If $\mu<\mu_{0} \equiv \frac{2-\sqrt{2}}{2}$, there exists $e_{0}<\mu_{0}-\mu$ ( $e_{0}$ is the same parameter appearing in (2.1)) such that, for $|e| \leq e_{0}, \varepsilon_{0}(\vec{k})-\mu=e$ defines a convex curve $\Sigma^{(0)}(e)$ encircling the origin, which can be represented in polar coordinates as $\vec{p}=u_{0}(\theta, e) \vec{e}_{r}(\theta)$ with $\vec{e}_{r}(\theta)=(\cos \theta, \sin \theta)$. Moreover $u_{0}(\theta, e) \geq c^{0}>0$ and, if $r_{0}(\theta, e)$ is the curvature radius,

$$
r_{0}(\theta, e)^{-1} \geq c^{0}>0
$$

Note that the symmetry property $\varepsilon_{0}(\vec{k})=\varepsilon_{0}(-\vec{k})$ implies that the curves $\Sigma^{(0)}(e)$ are symmetric by reflection with respect to the origin.

2. If $|e| \leq e_{0}$ and $\vec{p}=u_{0}(\theta, e) \vec{e}_{r}(\theta)$, then

$$
0<c_{1}^{0} \leq \vec{\nabla} \varepsilon_{0}(\vec{p}) \cdot \vec{e}_{r}(\theta) \leq c_{2}^{0} .
$$


3. Given $e$ as in item 1$)$ and $\vec{k}_{1}, \ldots, \vec{k}_{2 n} \in \Sigma^{(0)}(e), n \leq 4$, then

$$
\left|\sum_{i=1}^{2 n} \vec{k}_{i}\right|<2 \pi
$$

We shall refer to this property by saying that "umklapp processes with $n \leq 4$ quasi-particles are not allowed".

Remark. The validity of property (3) can be checked by noting that: if $\mu_{1}<\mu_{2}$ the surface $\Sigma_{\mu_{1}}^{(0)}(0)$ corresponding to chemical potential $\mu_{1}$ is completely enclosed into the surface $\Sigma_{\mu_{2}}^{(0)}(0)$ corresponding to chemical potential $\mu_{2}$; at $\mu$ and $e$ fixed and $n \leq 4$, the l.h.s. of (2.44) is maximized by $8 \arccos (1-\mu-e)$, obtained in correspondence of the choice $\vec{k}_{i}=(\arccos (1-\mu-e), 0), \forall i=1, \ldots, 2 n$; the condition $\mu+e_{0}<(2-\sqrt{2}) / 2$ is equivalent to $8 \arccos \left(1-\mu-e_{0}\right)<2 \pi$.

We call $\Sigma_{F}^{(0)} \equiv \Sigma^{(0)}(0)$ the free Fermi surface and we put $u_{0}(\theta, 0) \vec{e}_{r}(\theta)=$ $\vec{p}_{F}^{(0)}(\theta)$ and $u_{0}(\theta) \equiv u_{0}(\theta, 0)=\left|\vec{p}_{F}^{(0)}(\theta)\right|$.

The key remark is that, if $(2.38)$ is true for $|U| \leq U_{0}$ with $U_{0}$ small enough, for any $h_{\beta} \leq h \leq 0$, then the same properties (1)-(3) above still hold (with slightly modified constants) for the Fermi surfaces corresponding to the dispersion relations $\varepsilon_{h}(\vec{k})$.

Lemma 2.1. Let us assume that (2.38) is satisfied for $h_{\beta} \leq h \leq 0$ and for $|U| \leq U_{0}$, with $c_{0} \equiv\left|h_{\beta}\right| U_{0}$ small enough. Then there exist constants $\bar{e}, c, c_{1}, c_{2}$ such that the following properties are true.

1. If $|e| \leq \bar{e}, \varepsilon_{h}(\vec{k})-\mu=e$ defines a convex curve $\Sigma^{(h)}(e)$, encircling the origin and symmetric by reflection with respect to it, which can be represented in polar coordinates as $\vec{p}=u_{h}(\theta, e) \vec{e}_{r}(\theta)$. Moreover $u_{h}(\theta, e) \geq c>0$ and, if $r_{h}(\theta, e)$ is the curvature radius,

$$
r_{h}(\theta, e)^{-1} \geq c>0 .
$$

2. If $|e| \leq \bar{e}$ and $\vec{p}=u_{h}(\theta, e) \vec{e}_{r}(\theta)$, then

$$
0<c_{1} \leq \vec{\nabla} \varepsilon_{h}(\vec{p}) \cdot \vec{e}_{r}(\theta) \leq c_{2} .
$$

3. If $\mu<\mu_{0} \equiv \frac{2-\sqrt{2}}{2}$ and $|e| \leq \bar{e}$, then

$$
n \leq 4, \quad \vec{k}_{i} \in \Sigma^{(h)}(e), i=1, \ldots, 2 n, \quad \Rightarrow \quad\left|\sum_{i=1}^{2 n} \vec{k}_{i}\right|<2 \pi .
$$

Remark. Lemma 2.1 says that, under the smallness and smoothness properties (2.38) and under the symmetry assumption (2.40), the qualitative and quantitative properties of the dispersion relation on scale $h$ are the same as the free one. From the proof below it will become clear that, if $U_{0}$ is small enough, then the constants $\bar{e}, c, c_{1}, c_{2}$ can be chosen equal to: $\bar{e}=e_{0} / 2, c=c^{0} / 2, c_{1}=c_{1}^{0} / 2$ and $c_{2}=3 c_{2}^{0} / 2$. 
Proof. Given $h_{\beta} \leq h \leq 0$, we can write $\varepsilon_{h}(\vec{k})=\varepsilon_{0}(\vec{k})+\sum_{j=h+1}^{0}\left(\varepsilon_{j-1}(\vec{k})-\varepsilon_{j}(\vec{k})\right)$. From this identity and the inductive assumption (2.38) we soon find, if $c_{0} \leq 1$,

$$
\begin{gathered}
\left|\varepsilon_{h}(\vec{k})-\varepsilon_{0}(\vec{k})\right| \leq C_{0}|U| \sum_{j=h_{\vec{k}}}^{0}|j| \gamma^{2 j} \leq C_{0}^{\prime}|U|, \\
\left|\vec{\nabla} \varepsilon_{h}(\vec{k})-\vec{\nabla} \varepsilon_{0}(\vec{k})\right| \leq 2 C_{1}|U| \sum_{j=h_{\vec{k}}}^{0} \gamma^{j}|j| \leq C_{1}^{\prime}|U|, \\
\left|\partial_{k_{i} k_{r}}^{2} \varepsilon_{h}(\vec{k})-\partial_{k_{i} k_{r}}^{2} \varepsilon_{0}(\vec{k})\right| \leq C_{2}|U|\left[|U| \sum_{j=h_{\vec{k}}+3}^{0}|j|+\sum_{j=h+1}^{h_{\vec{k}}+3}|j|\right] \leq 4 C_{2} c_{0},
\end{gathered}
$$

where $h_{\vec{k}}=\min \left\{j \geq h+1: C_{j}^{-1}\left(\pi \beta^{-1}, \vec{k}\right)>0\right\}$ and we used that $C_{j}^{-1}\left(\pi \beta^{-1}, \vec{k}\right)=1$ if $j \geq h_{\vec{k}}+3$ and that $\sum_{j=h+1}^{h_{\vec{k}}+3} \leq 3$. The first two bounds in (2.48) show that $\varepsilon_{h}(\vec{k})$ and $\vec{\nabla} \varepsilon_{h}(\vec{k})$ are close within $O(U)$ and $O\left(U^{2}\right)$ to $\varepsilon_{0}(\vec{k})$ and $\vec{\nabla} \varepsilon_{0}(\vec{k})$ respectively. This properties and the validity of properties (1)-(3) in the unperturbed case $h=0$ guarantee that the equation $\varepsilon_{h}(\vec{k})-\mu=e$ can be inverted for $h<0$ if $e$ is small enough (as it follows from an application of implicit function theorem). Then the set of vectors $\Sigma^{(h)}(e)$ defines a closed curve enclosing the origin (and symmetric around it, because of the remark after $(2.41))$, close to $\Sigma^{(0)}(e)$ within $O(U)$. Also, the third bound in (2.48) implies that the second derivatives of $\varepsilon_{h}(\vec{k})$ are close to the second derivatives of $\varepsilon_{0}(\vec{k})$, if $c_{0}$ small enough. This means that $\Sigma^{(h)}(e)$ is convex (since so $\Sigma^{(0)}(e)$ is) and $r_{h}(\theta, e)=r_{0}(\theta, e)+O\left(c_{0}^{2}\right)$ (because the curvature radius $r_{h}(\theta, e)$ is computed in terms of the first two derivatives of $\left.\varepsilon_{h}(\vec{k})\right)$ and the lemma is proved.

In analogy with the definitions corresponding to the unperturbed dispersion relation $\varepsilon_{0}(\vec{k})$, we call $\Sigma_{F}^{(h)} \equiv \Sigma^{(h)}(0)$ the Fermi surface on scale $h$ and we put $u_{h}(\theta, 0) \vec{e}_{r}(\theta)=\vec{p}_{F}^{(h)}(\theta)$ and $u_{h}(\theta) \equiv u_{h}(\theta, 0)=\left|\vec{p}_{F}^{(h)}(\theta)\right|$.

We conclude this section by listing some more properties of $E_{h}(\mathbf{k})$ and of $\hat{g}^{(h)}(\mathbf{k})$ following from the inductive assumptions (2.38). First of all, by proceeding as in the proof of Lemma 2.1, we see that (2.38) imply that

$$
\begin{aligned}
\left|E_{h}(\mathbf{k})-E_{0}(\mathbf{k})\right| & \leq C_{0}^{\prime}|U| \\
\left|\partial_{k_{i}} E_{h}(\mathbf{k})-\partial_{k_{i}} E_{0}(\mathbf{k})\right| & \leq C_{1}^{\prime}|U| \\
\left|\partial_{k_{i_{1}}} \partial_{k_{i_{2}}} E_{h}(\mathbf{k})-\partial_{k_{i_{1}}} \partial_{k_{i_{2}}} E_{0}(\mathbf{k})\right| & \leq C_{2}^{\prime} c_{0} \\
\left|\partial_{k_{i_{1}}} \cdots \partial_{k_{i_{n}}} E_{h}(\mathbf{k})-\partial_{k_{i_{1}}} \cdots \partial_{k_{i_{n}}} E_{0}(\mathbf{k})\right| & \leq C_{n}^{\prime}|U||h| \gamma^{(2-n) h}, \quad n \geq 3
\end{aligned}
$$

with $c_{0} \equiv\left|h_{\beta}\right| U_{0}$ small enough.

Finally, let us consider the propagator $\hat{g}^{(h)}(\mathbf{k})$ in (2.28). By putting $E_{h-1}(\mathbf{k})$ $=E_{h}(\mathbf{k})+\left(E_{h-1}(\mathbf{k})-E_{h}(\mathbf{k})\right)$ and by expanding $E_{h}(\mathbf{k})$ at first order in $k_{0}$, we see 
that the propagator $\hat{g}^{(h)}(\mathbf{k})$ in $(2.28)$ can be rewritten as

$$
\hat{g}^{(h)}(\mathbf{k})=\frac{f_{h}(\mathbf{k})}{-i k_{0}\left[1+a_{h}(\vec{k})\right]+\varepsilon_{h}(\vec{k})+r_{h}(\mathbf{k})-\mu},
$$

where, if $\partial_{k_{0}}$ is defined as in (1.8),

$$
a_{h}(\vec{k})=i \partial_{k_{0}} E_{h}\left(-\frac{\pi}{\beta}, \vec{k}\right) .
$$

By the second of (2.40), we see that $a_{h}(\vec{k})$ is real. Moreover both $a_{h}$ and the rest $r_{h}$ can be bounded using (2.38) and (2.49); we find that there exists $C>0$ such that $\left|a_{h}(\vec{k})\right| \leq C|U|$ and $\left|r_{h}(\mathbf{k})\right| \leq C|U||h| \gamma^{2 h}$ for any $\mathbf{k}$ in the support of $f_{h}(\mathbf{k})$. This implies in particular that, under the inductive assumption (2.38), the scale $h_{\beta}$ defined in (2.33) is finite and larger than $\left[\log _{\gamma}\left(\pi / 2 e_{0} \beta\right)\right]$, if $U$ is small enough. Moreover, by using the definition of $f_{h}(\mathbf{k})$ in $(2.29)$, one can easily see that there exists $K>0$ such that

$$
f_{h}(\mathbf{k}) \neq 0 \Rightarrow K^{-1} e_{0} \gamma^{h} \leq\left|k_{0}\right|+\left|\varepsilon_{h}(\vec{k})-\mu\right| \leq K e_{0} \gamma^{h} .
$$

In particular, if $f_{h}(\mathbf{k}) \neq 0$, we can write $\vec{k}=u_{h}(\theta, e) \vec{e}_{r}(\theta)$ with $e=\varepsilon_{h}(\vec{k})-\mu$ and $|e| \leq K \gamma^{h} e_{0}$.

\subsection{The sector decomposition}

The smoothness properties of $E_{h}(\mathbf{k})$ and the symmetry and convexity properties of the Fermi surface described in previous subsection allow for a further decomposition of the propagator $\hat{g}^{(h)}(\mathbf{k})$ that is convenient for explicitly performing the bounds on the kernels of $\mathcal{V}^{(h)}$ : as in $[\mathrm{BGM}]$ we decompose the field $\psi^{(\leq h)}$, by slicing the support of $C_{h}^{-1}(\mathbf{k})$ as follows. As in Section 2.3 of [BGM], we introduce the angles $\theta_{h, \omega}=\pi\left(\omega+\frac{1}{2}\right) \gamma^{h / 2}$, with $\omega$ an integer in the set $O_{h}=\left\{0,1, \ldots, \gamma^{-(h-1) / 2}-1\right\}$ (recall that $\gamma=4$ ). Correspondingly we introduce the functions $\zeta_{h, \omega}(\theta)$ with the properties:

$$
\begin{aligned}
& \left\|\theta-\theta_{h, \omega}\right\|<\frac{\pi}{4} \gamma^{h / 2} \Rightarrow \zeta_{h, \omega}(\theta)=1 \\
& \left\|\theta-\theta_{h, \omega}\right\|>\frac{3 \pi}{4} \gamma^{h / 2} \Rightarrow \zeta_{h, \omega}(\theta)=0 \\
& \sum_{\omega \in O_{h}} \zeta_{h, \omega}(\theta)=1, \quad \forall \theta \in \mathbb{T}^{1}
\end{aligned}
$$

where $\|\cdot\|$ is the usual distance on $\mathbb{T}^{1}$.

We also introduce the support function $F_{h, \omega}(\mathbf{k})=f_{h}(\mathbf{k}) \zeta_{h, \omega}(\theta)$, where, if $\mathbf{k}=\left(k_{0}, \vec{k}\right)$, then $\theta$ is the polar angle of $\vec{k}$. We shall call the functions $F_{h, \omega}(\mathbf{k})$ the anisotropic support functions and the indices $\omega \in O_{h}$ the anisotropic sector indices (the name recalls the fact that the support of $F_{h, \omega}(0, \vec{k})$ is a geometric set that is "wider" than "thick"; it is $O\left(\gamma^{h / 2}\right)$ "wide" in the direction tangential to the Fermi surface and $O\left(\gamma^{h}\right)$ "thick" in the normal direction). 
Given any $\mathbf{k}$ belonging to the support of $F_{h, \omega}(\mathbf{k})$, we put

$$
\vec{k}=\vec{p}_{F}^{(h)}\left(\theta_{h, \omega}\right)+k_{1}^{\prime} \vec{n}_{h}\left(\theta_{h, \omega}\right)+k_{2}^{\prime} \vec{\tau}_{h}\left(\theta_{h, \omega}\right)=\vec{p}_{F}^{(h)}\left(\theta_{h, \omega}\right)+\vec{k}^{\prime}
$$

where, putting $\vec{e}_{t}(\theta)=(-\sin \theta, \cos \theta)$, the vectors $\vec{\tau}_{h}(\theta)$ and $\vec{n}_{h}(\theta)$ are defined as

$$
\begin{aligned}
& \vec{\tau}_{h}(\theta)=\frac{d \vec{p}_{F}^{(h)}(\theta)}{d \theta}\left|\frac{d \vec{p}_{F}^{(h)}(\theta)}{d \theta}\right|^{-1}=\frac{u_{h}^{\prime}(\theta) \vec{e}_{r}(\theta)+u_{h}(\theta) \vec{e}_{t}(\theta)}{\sqrt{u_{h}^{\prime}(\theta)^{2}+u_{h}(\theta)^{2}}}, \\
& \vec{n}_{h}(\theta)=\frac{u_{h}(\theta) \vec{e}_{r}(\theta)-u_{h}^{\prime}(\theta) \vec{e}_{t}(\theta)}{\sqrt{u_{h}^{\prime}(\theta)^{2}+u_{h}(\theta)^{2}}} .
\end{aligned}
$$

Using (2.52) and (2.53) it is easy to realize that $\left|k_{1}^{\prime}\right| \leq C \gamma^{h}$ and $\left|k_{2}^{\prime}\right| \leq C \gamma^{h / 2}$ for some constant $C$; see Lemma 7.3 of $[\mathrm{BGM}]$ for details.

Using the decomposition (2.54), we can rewrite

$$
\psi_{\mathbf{x}, \sigma}^{(h) \pm} \equiv \sum_{\omega \in O_{h}} e^{ \pm i \vec{p}_{F}^{(h)}\left(\theta_{h, \omega}\right) \vec{x}} \psi_{\mathbf{x}, \sigma, \omega}^{(h) \pm} \quad, \quad P\left(d \psi^{(h)}\right)=\prod_{\omega \in O_{h}} P\left(d \psi_{\omega}^{(h)}\right)
$$

where $P\left(d \psi_{\omega}^{(h)}\right)$ is the Grassmannian integration with propagator

$$
g_{\omega}^{(h)}(\mathbf{x})=\frac{1}{\beta} \sum_{k_{0} \in \mathcal{D}_{\beta}} \int_{-\pi}^{\pi} \int_{-\pi}^{\pi} \frac{d \vec{k}^{\prime}}{(2 \pi)^{2}} e^{-i\left(k_{0} x_{0}+\vec{k}^{\prime} \vec{x}\right)} \frac{F_{h, \omega}\left(\mathbf{k}^{\prime}+\mathbf{p}_{F}^{(h)}\left(\theta_{h, \omega}\right)\right)}{-i k_{0}+E_{h-1}\left(\mathbf{k}^{\prime}+\mathbf{p}_{F}^{(h)}\left(\theta_{h, \omega}\right)\right)-\mu} .
$$

where we defined $\mathbf{p}_{F}^{(h)}\left(\theta_{h, \omega}\right) \stackrel{\text { def }}{=}\left(0, \vec{p}_{F}^{(h)}\left(\theta_{h, \omega}\right)\right)$. We insert the decomposition $(2.56)$ into the r.h.s. of $(2.30)$ :

$$
\int P_{E_{h-1}, f_{h}^{-1}}\left(d \psi^{(h)}\right) \exp \left\{-\hat{\mathcal{V}}^{(h)}\left(\psi_{\mathbf{x}, \sigma}^{(\leq h-1) \pm}+\sum_{\omega \in O_{h}} e^{ \pm i \vec{p}_{F}^{(h)}\left(\theta_{h, \omega}\right) \vec{x}} \psi_{\mathbf{x}, \sigma, \omega}^{(h) \pm}\right)\right\},
$$

and in this way we induce a decomposition of the kernels of $\mathcal{V}^{(h-1)}$ into a sum of contributions labelled by the choices of the sector labels of the integrated fields $\psi_{\mathbf{x}, \sigma, \omega}^{(h) \pm}$, see next section for a description of this further decomposition of the kernels of $\mathcal{V}^{(h-1)}$

The bounds on the (decomposed) kernels of $\mathcal{V}^{(h-1)}$ are based on the following key bound on the asymptotic behavior of $g_{\omega}^{(h)}(\mathbf{x})$.

Lemma 2.2. Let us assume that the bounds (2.38) are valid and that $c_{0} \equiv\left|h_{\beta}\right| U_{0}$ is small enough. Given $h_{\beta} \leq h \leq 0$ and $\omega \in O_{h}$, let us put

$$
\vec{x}=x_{1}^{\prime} \vec{n}_{h}\left(\theta_{h, \omega}\right)+x_{2}^{\prime} \vec{\tau}_{h}\left(\theta_{h, \omega}\right)
$$

Then, given $M \geq 1$ and $N \geq 2$, there exists a constant $C_{M, N}$ such that

$$
\left|g_{\omega}^{(h)}(\mathbf{x})\right| \leq \frac{C_{M, N} \gamma^{\frac{3}{2} h}}{\left[1+\left(\gamma^{h}\left|d_{\beta}\left(x_{0}\right)\right|+\gamma^{h}\left|x_{1}^{\prime}\right|\right)^{M}\right]\left[1+\gamma^{-h}\left(\gamma^{h}\left|x_{2}^{\prime}\right|\right)^{N}\right]}
$$

where $d_{\beta}\left(x_{0}\right)=\beta \pi^{-1} \sin \left(\pi \beta^{-1} x_{0}\right)$. 
Remark. The bound (2.60) implies that

$$
\int d \mathbf{x}|\mathbf{x}|^{j} \cdot\left|g_{\omega}^{(h)}(\mathbf{x})\right| \leq C_{j} \gamma^{-(1+j) h}, \quad j \geq 0,
$$

that will be widely used in the following. In order to prove $(2.61)$, it is sufficient to consider the integral obtained by substituting $|\mathbf{x}|^{j}$ with $\left(\sqrt{\left|d_{\beta}\left(x_{0}\right)\right|^{2}+\left|x_{1}^{\prime}\right|^{2}}\right)^{j_{1}}$. $\left|x_{2}^{\prime}\right|^{j_{2}}, j_{1}+j_{2}=j$. This integral can be bounded by choosing $M \geq 3+j_{1}$ and $N=2+j_{2}$ in (2.60) and by doing the rescalings $x_{0} \rightarrow \gamma^{-h} x_{0}, x_{1}^{\prime} \rightarrow \gamma^{-h} x_{1}^{\prime}$ and $x_{2}^{\prime} \rightarrow \gamma^{-h\left(j_{2}+1\right) /\left(j_{2}+2\right)} x_{2}^{\prime}$; one gets a bound proportional to $\gamma^{-j_{1} h-\alpha_{j_{2}} h}$, with $\alpha_{j}=1 / 2+(j+1)^{2} /(j+2) \leq j+1$.

Proof. The key remark in order to get the bound (2.60) is the following. If $\mathbf{k}=$ $\left(k_{0}, \vec{k}\right)$ belongs to the support of $F_{h, \omega}(\mathbf{k}), \omega \in O_{h}$, and we write $\vec{k}=\vec{p}_{F}^{(h)}\left(\theta_{h, \omega}\right)+$ $k_{1}^{\prime} \vec{n}_{h}\left(\theta_{h, \omega}\right)+k_{2}^{\prime} \vec{\tau}_{h}\left(\theta_{h, \omega}\right)$, then

$$
\left|\frac{\partial E_{h-1}(\mathbf{k})}{\partial k_{2}^{\prime}}\right| \leq C \gamma^{\frac{h}{2}}
$$

for some constant $C$. In fact, if $\mathbf{k}=\left(k_{0}, \vec{k}\right)$ belongs to the support of $F_{h, \omega}(\mathbf{k})$, we can write $E_{h-1}(\mathbf{k})=\varepsilon_{h}(\vec{k})+\left(E_{h-1}(\mathbf{k})-E_{h}(\mathbf{k})\right)+\left(E_{h}(\mathbf{k})-\varepsilon_{h}(\vec{k})\right)$ where, by the properties described in previous section, $\mid \partial_{k_{2}^{\prime}}\left[\left(E_{h-1}(\mathbf{k})-E_{h}(\mathbf{k})\right)+\left(E_{h}(\mathbf{k})-\right.\right.$ $\left.\left.\varepsilon_{h}(\vec{k})\right)\right] \mid \leq C \gamma^{h}$. Moreover it is easy to prove that $\partial_{k_{2}^{\prime}} \varepsilon_{h}(\vec{k})=O\left(\gamma^{h / 2}\right)$. In fact we have $\vec{\nabla} \varepsilon_{h}(\vec{k})=\left|\vec{\nabla} \varepsilon_{h}(\vec{k})\right| \vec{n}_{h}(\theta, e)$ where $\left|\vec{\nabla} \varepsilon_{h}(\vec{k})\right|=O(1), e=\varepsilon_{h}(\vec{k})-\mu=O\left(\gamma^{h}\right)$, $\theta$ is the polar angle of $\vec{k}$ and $\vec{n}_{h}(\theta, e)$ is the outgoing normal vector at $\Sigma^{(h)}(e)$ in $\vec{k}$. Furthermore $\left\|\theta-\theta_{h, \omega}\right\|=O\left(\gamma^{h / 2}\right)$ so that

$$
\begin{aligned}
\frac{\partial \varepsilon_{h}(\vec{k})}{\partial k_{2}^{\prime}} & =\left|\vec{\nabla} \varepsilon_{h}(\vec{k})\right| \vec{n}_{h}(\theta, e) \cdot \vec{\tau}_{h}\left(\theta_{h, \omega}\right)=\left|\vec{\nabla} \varepsilon_{h}(\vec{k})\right| \vec{n}_{h}(\theta) \cdot \vec{\tau}_{h}\left(\theta_{h, \omega}\right)+O\left(\gamma^{h}\right)= \\
& =\left|\vec{\nabla} \varepsilon_{h}(\vec{k})\right| \sin \left(\theta-\theta_{h, \omega}\right)+O\left(\gamma^{h}\right)=O\left(\gamma^{h / 2}\right)
\end{aligned}
$$

and (2.62) follows.

The bound (2.60) is simply obtained by integration by parts and dimensional bounds on the integrand and on the measure of the support. First of all, note that by the compact support properties of $F_{h, \omega}(\mathbf{k})$ it holds that $\left|g_{\omega}^{(h)}(\mathbf{x})\right| \leq C \gamma^{\frac{3}{2} h}$, for some constant $C$. In order to bound $\left|\left(x_{2}^{\prime}\right)^{N} g_{\omega}^{(h)}(\mathbf{x})\right|$ with $N \geq 2$, by integrating by parts with respect to $k_{2}^{\prime}$, we rewrite:

$$
\begin{gathered}
\left|\left(x_{2}^{\prime}\right)^{N} g_{\omega}^{(h)}(\mathbf{x})\right|=\mid \frac{1}{\beta} \sum_{k_{0} \in \mathcal{D}_{\beta}} \int \frac{d \vec{k}^{\prime}}{(2 \pi)^{2}} e^{-i\left(k_{0} x_{0}+\vec{k}^{\prime} \vec{x}\right)} \partial_{k_{2}^{\prime}}^{N} \\
\cdot\left[\frac{F_{h, \omega}\left(\mathbf{k}^{\prime}+\mathbf{p}_{F}^{(h)}\left(\theta_{h, \omega}\right)\right)}{-i k_{0}+E_{h-1}\left(\mathbf{k}^{\prime}+\mathbf{p}_{F}^{(h)}\left(\theta_{h, \omega}\right)\right)-\mu}\right] \mid .
\end{gathered}
$$

Using (2.62), (2.49) and the fact that the $n$-th order derivative of $\zeta_{h, \omega}(\theta)$ is of order $\gamma^{-n h / 2}$, it is easy to see that $\partial_{k_{2}^{\prime}}^{N}\left[\frac{F_{h, \omega}\left(\mathbf{k}^{\prime}+\mathbf{p}_{F}^{(h)}\left(\theta_{h, \omega}\right)\right)}{-i k_{0}+E_{h-1}\left(\mathbf{k}^{\prime}+\mathbf{p}_{F}^{(h)}\left(\theta_{h, \omega}\right)\right)-\mu}\right]=O\left(\gamma^{-N h}\right)$ 
for $N \geq 2$ and any $\mathbf{k}^{\prime}$ in the support of $F_{h, \omega}\left(\mathbf{k}^{\prime}+\mathbf{p}_{F}^{(h)}\left(\theta_{h, \omega}\right)\right)$; this implies that $\left|\left(x_{2}^{\prime}\right)^{N} g_{\omega}^{(h)}(\mathbf{x})\right| \leq C_{N} \gamma^{\frac{3}{2} h} \gamma^{-h(N-1)}$ for some constant $C_{N}$.

Similarly, using the bounds (2.49), we find that, given $N \geq 0$,

$$
\left|\left[d_{\beta}\left(x_{0}\right)\right]^{N} g_{\omega}^{(h)}(\mathbf{x})\right| \leq C_{N} \gamma^{\frac{3}{2} h} \gamma^{-N h}, \quad\left|\left(x_{1}^{\prime}\right)^{N} g_{\omega}^{(h)}(\mathbf{x})\right| \leq C_{N} \gamma^{\frac{3}{2} h} \gamma^{-N h} .
$$

Combining (2.65) with the analogue bounds on $\left|g_{\omega}^{(h)}(\mathbf{x})\right|$ and $\left|\left(x_{2}^{\prime}\right)^{N} g_{\omega}^{(h)}(\mathbf{x})\right|$ we find (2.60).

Note that $g_{\omega}^{(h)}(\mathbf{x})$ is approximately odd in $\mathbf{x}$ : in fact $g_{\omega}^{(h)}(\mathbf{0})$ admits an improved dimensional estimate with respect to the bound (2.60), as expressed by the following Lemma.

Lemma 2.3. Let us fix $h \leq 0$ and $\omega \in O_{h}$. Then

$$
\left|g_{\omega}^{(h)}(\mathbf{0})\right| \leq C \gamma^{\frac{5}{2} h} .
$$

Remark. Note that the bound (2.66) on the size of $g_{\omega}^{(h)}(\mathbf{0})$ is $\gamma^{h}$ smaller than the bound on the size of $g_{\omega}^{(h)}(\mathbf{x})$, see $(2.60)$.

Proof. The propagator $g_{\omega}^{(h)}(\mathbf{0})$ can be written as

$$
g_{\omega}^{(h)}(\mathbf{0})=\int d \theta \zeta_{h, \omega}(\theta) \int d k_{0} \rho d \rho \frac{f_{h}\left(k_{0}, \rho \vec{e}_{r}(\theta)\right)}{-i k_{0}[1+a(\theta)]+e_{\theta}(\rho)+O\left(c_{0} \gamma^{2 h}\right)}
$$

where, referring to $(2.50), a(\theta)=a_{h}\left(u_{h}(\theta) \vec{e}_{r}(\theta)\right)$ and $e_{\theta}(\rho) \stackrel{\text { def }}{=} \varepsilon_{h}\left(\rho \vec{e}_{r}(\theta)\right)-\varepsilon_{h}\left(u_{h}(\theta)\right.$ $\vec{e}_{r}(\theta)$ ) (note that $e_{\theta}\left(u_{h}(\theta)\right)=0$ and $e_{\theta}^{\prime}\left(u_{h}(\theta)\right)=O(1)$ uniformly in $\theta$ ). The bound on the error term in the denominator in (2.67) comes from the bounds on $r_{h}(\mathbf{k})$ and $a_{h}(\vec{k})$ discussed after (2.50). Similarly we find that $f_{h}\left(k_{0}, \rho \vec{e}_{r}(\theta)\right)=$ $\tilde{f}_{h}\left(\sqrt{k_{0}^{2}[1+a(\theta)]^{2}+e_{\theta}(\rho)^{2}}\right)+f_{h}^{R}\left(k_{0}, \rho \vec{e}_{r}(\theta)\right)$, where

$$
\tilde{f}_{h}(t)=H_{0}\left(\gamma^{-h} t\right)-H_{0}\left(\gamma^{-h+1} t\right)
$$

and $f_{h}^{R}\left(k_{0}, \rho \vec{e}_{r}(\theta)\right)$ is an $O\left(\gamma^{h}\right)$ function, vanishing outside a region slightly larger than the support of $f_{h}\left(k_{0}, \rho \vec{e}_{r}(\theta)\right)$. Replacing in $(2.67) f_{h}$ with $\tilde{f}_{h}+f_{h}^{R}$ and using that the error term in the denominator is $O\left(c_{0} \gamma^{2 h}\right)$, we find that

$$
g_{\omega}^{(h)}(\mathbf{0})=\int d \theta \zeta_{h, \omega}(\theta) \int d k_{0} \rho d \rho \frac{\tilde{f}_{h}\left(\sqrt{k_{0}^{2}[1+a(\theta)]^{2}+e_{\theta}(\rho)^{2}}\right)}{-i k_{0}[1+a(\theta)]+e_{\theta}(\rho)}+R_{\omega}^{(h)},
$$

where the rest $R_{\omega}^{(h)}$ is dimensionally bounded by $\left|R_{\omega}^{(h)}\right| \leq c \gamma^{\frac{5}{2} h}$.

Now, for any fixed $\theta$, we can use Dini's theorem to invert the relation $e_{\theta}(\rho)=e$ into $\rho=\rho(e)$, and we can rewrite the first integral in the r.h.s. of (2.69) as

$$
\int d \theta \zeta_{h, \omega}(\theta) \int d k_{0} \rho(e) d e \frac{1}{e_{\theta}^{\prime}(\rho(e))} \frac{\tilde{f}_{h}\left(\sqrt{k_{0}^{2}[1+a(\theta)]^{2}+e^{2}}\right)}{-i k_{0}[1+a(\theta)]+e} .
$$


Note that on the support of $\tilde{f}_{h}$ we have $e=O\left(\gamma^{h}\right)$, so that we can rewrite $\rho(e)=$ $u_{h}(\theta)+O\left(\gamma^{h}\right)$ and $e_{\theta}^{\prime}(\rho(e))=e_{\theta}^{\prime}\left(u_{h}(\theta)\right)+O\left(\gamma^{h}\right)$, where $u_{h}(\theta)$ and $e_{\theta}^{\prime}\left(u_{h}(\theta)\right)$ are bounded below and above by positive $O(1)$ constants, uniformly in $\theta$. Then $(2.70)$ is equal to

$$
\int d \theta \zeta_{h, \omega}(\theta) u_{h}(\theta) \frac{1}{e_{\theta}^{\prime}\left(u_{h}(\theta)\right)} \int d k_{0} d e \frac{\tilde{f}_{h}\left(\sqrt{k_{0}^{2}[1+a(\theta)]^{2}+e^{2}}\right)}{-i k_{0}+e}+O\left(\gamma^{\frac{5}{2} h}\right)
$$

and, since the first integral is zero by oddity, the Lemma is proved.

In order to perform the inductive bounds, in the following it will also be convenient to introduce, besides the anisotropic sector functions, the isotropic ones, defined as follows. We introduce the angles $\bar{\theta}_{h, \bar{\omega}}=\pi\left(\bar{\omega}+\frac{1}{2}\right) \gamma^{h}$, with $\bar{\omega}$ an integer in the set $\bar{O}_{h}=\left\{0,1, \ldots, \gamma^{-(h-1)}-1\right\}$, and the functions $\bar{\zeta}_{h, \bar{\omega}}(\theta)$ with the properties:

$$
\begin{aligned}
& \left\|\theta-\bar{\theta}_{h, \bar{\omega}}\right\|<\frac{\pi}{4} \gamma^{h} \Rightarrow \bar{\zeta}_{h, \bar{\omega}}(\theta)=1 \\
& \left\|\theta-\bar{\theta}_{h, \bar{\omega}}\right\|>\frac{3 \pi}{4} \gamma^{h} \Rightarrow \bar{\zeta}_{h, \bar{\omega}}(\theta)=0 \\
& \sum_{\bar{\omega} \in \bar{O}_{h}} \bar{\zeta}_{h, \bar{\omega}}(\theta)=1, \quad \forall \theta \in \mathbb{T}^{1} .
\end{aligned}
$$

We also introduce the support functions $\bar{F}_{h, \bar{\omega}}(\mathbf{k})=f_{h}(\mathbf{k}) \bar{\zeta}_{h, \bar{\omega}}(\theta)$, to be called the isotropic support functions. From now on we shall use the convention that

we shall denote all the quantities associated with the isotropic sectors by symbols obtained by overlining the symbols for the corresponding quantities associated with the anisotropic sectors.

Given any $\mathbf{k}$ belonging to the support of $\bar{F}_{h, \bar{\omega}}(\mathbf{k})$, we put

$$
\vec{k}=\vec{p}_{F}^{(h)}\left(\bar{\theta}_{h, \bar{\omega}}\right)+\vec{k}^{\prime}
$$

where $\vec{k}^{\prime}=O\left(\gamma^{h}\right)$. Correspondingly we can decompose $g^{(h)}(\mathbf{k})$ into a sum of isotropic propagators:

$$
\begin{aligned}
g^{(h)}(\mathbf{x})= & \sum_{\bar{\omega} \in \bar{O}_{h}} e^{i \vec{p}_{F}^{(h)}\left(\bar{\theta}_{h, \bar{\omega}}\right) \vec{x}} \bar{g}_{\bar{\omega}}^{(h)}(\mathbf{x}) \\
\bar{g}_{\bar{\omega}}^{(h)}(\mathbf{x})= & \frac{1}{\beta} \sum_{k_{0} \in \mathcal{D}_{\beta}} \int_{-\pi}^{\pi} \int_{-\pi}^{\pi} \frac{d \vec{k}^{\prime}}{(2 \pi)^{2}} e^{-i\left(k_{0} x_{0}+\vec{k}^{\prime} \vec{x}\right)} \\
& \cdot \frac{\bar{F}_{h, \bar{\omega}}\left(\mathbf{k}^{\prime}+\overline{\mathbf{p}}_{F}^{(h)}\left(\bar{\theta}_{h, \bar{\omega}}\right)\right)}{-i k_{0}+E_{h-1}\left(\mathbf{k}^{\prime}+\overline{\mathbf{p}}_{F}^{(h)}\left(\bar{\theta}_{h, \bar{\omega}}\right)\right)-\mu}
\end{aligned}
$$

with $\bar{g}_{\bar{\omega}}^{(h)}(\mathbf{x})$ satisfying the following analogue of Lemma 2.2 and of Lemma 2.3 (to be proven via a repetition of the proof of Lemma 2.2 and Lemma 2.3). 
Lemma 2.4. Let us assume that the bounds (2.38) are valid and that $c_{0} \equiv\left|h_{\beta}\right| U_{0}$ is small enough. Given $h_{\beta} \leq h \leq 0$ and $\bar{\omega} \in \bar{O}_{h}$ and $N \geq 0$, there exists a constant $C_{N}$ such that

$$
\left|\bar{g}_{\bar{\omega}}^{(h)}(\mathbf{x})\right| \leq \frac{C_{N} \gamma^{2 h}}{1+\left(\gamma^{h}\left|d_{\beta}\left(x_{0}\right)\right|+\gamma^{h}|\vec{x}|\right)^{N}} .
$$

Remark. (2.75) implies the analogue of (2.61):

$$
\int d \mathbf{x}|\mathbf{x}|^{j} \cdot\left|\bar{g}_{\bar{\omega}}^{(h)}(\mathbf{x})\right| \leq C_{j} \gamma^{-(1+j) h}, \quad j \geq 0 .
$$

Note that the dimensional bound on the integral of an isotropic propagator is the same as the bound on the integral of an anisotropic one.

Lemma 2.5. Let us fix $h \leq 0$ and $\bar{\omega} \in \bar{O}_{h}$. Then

$$
\left|\bar{g}_{\bar{\omega}}^{(h)}(\mathbf{0})\right| \leq C \gamma^{3 h} \text {. }
$$

Remark. Note that the bound (2.77) on the size of $\bar{g}_{\bar{\omega}}^{(h)}(\mathbf{0})$ is $\gamma^{h}$ smaller than the bound on the size of $\bar{g}_{\bar{\omega}}^{(h)}(\mathbf{x})$, see $(2.75)$.

\subsection{The tree expansion}

In this section and in the two following ones we shall describe the expansion for the effective potential $\mathcal{V}^{(h)}$ and the inductive bounds we use to prove convergence of the expansion for the free energy, under the smoothness and smallness assumption (2.38) and under a smallness assumption on $\lambda_{h}(\mathbf{x})$ to be stated in a precise form below. In particular we shall summarize the bounds described in [BGM] in a form adapted to the present case and suitable for proving the improved dimensional bounds on $E_{h}(\mathbf{k})$ (i.e., allowing for an inductive proof of (2.38), see next chapter).

Our expansion of $\mathcal{V}^{(h)}, 0 \geq h \geq h_{\beta}$, is obtained by integrating iteratively the field variables of scale $j \geq h+1$ and sector index $\omega \in O_{j}$ (for the moment we do not consider isotropic sectors; they will be introduced in next chapter in order to optimize the bounds) and by applying at each step the localization procedure described above. The result can be expressed in terms of a tree expansion, very similar to the one described in $[\mathrm{BGM}]$. For completeness we list here the definition of trees. See Fig. 1 for an example of a possible tree appearing in the expansion for the effective potentials.

1) Let us consider the family of all trees which can be constructed by joining a point $r$, the root, with an ordered set of $n \geq 1$ points, the endpoints of the unlabeled tree (see Fig. 1), so that $r$ is not a branching point. $n$ will be called the order of the unlabeled tree and the branching points will be called the non trivial vertices. The unlabeled trees are partially ordered from the root to the endpoints in the natural way; we shall use the symbol $<$ to denote the partial order.

Two unlabeled trees are identified if they can be superposed by a suitable continuous deformation, so that the endpoints with the same index coincide. It is 


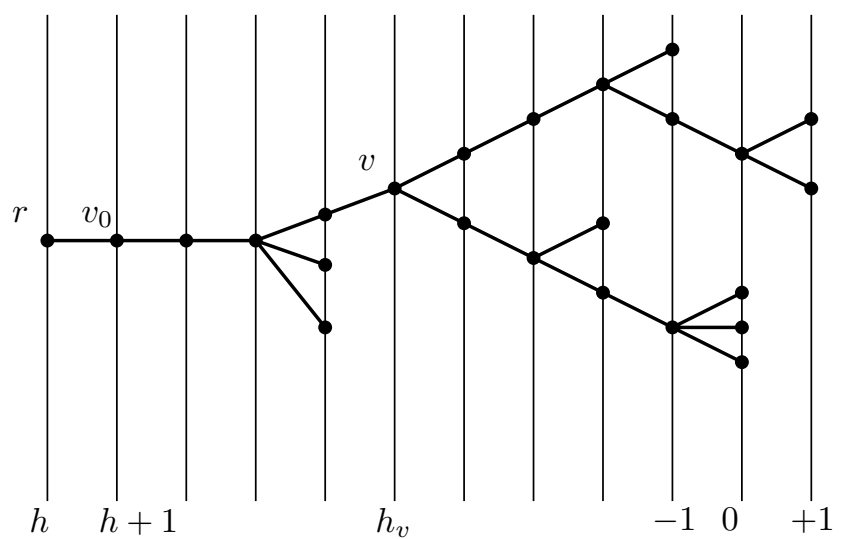

FiguRE 1. An example of tree.

then easy to see that the number of unlabeled trees with $n$ end-points is bounded by $4^{n}$.

We shall consider also the labelled trees (to be called simply trees in the following); they are defined by associating some labels with the unlabeled trees, as explained in the following items.

2) We associate a label $h \leq-1$ with the root and we denote by $\mathcal{T}_{h, n}$ the corresponding set of labelled trees with $n$ endpoints. Moreover, we introduce a family of vertical lines, labelled by an integer taking values in $[h, 1]$, and we represent any tree $\tau \in \mathcal{T}_{h, n}$ so that, if $v$ is an endpoint or a non trivial vertex, it is contained in a vertical line with index $h_{v}>h$, to be called the scale of $v$, while the root is on the line with index $h$. There is the constraint that, if $v$ is an endpoint, $h_{v}>h+1$.

The tree will intersect in general the vertical lines in set of points different from the root, the endpoints and the non trivial vertices; these points will be called trivial vertices. The set of the vertices of $\tau$ will be the union of the endpoints, the trivial vertices and the non trivial vertices. Note that, if $v_{1}$ and $v_{2}$ are two vertices and $v_{1}<v_{2}$, then $h_{v_{1}}<h_{v_{2}}$.

Moreover, there is only one vertex immediately following the root, which will be denoted $v_{0}$ and can not be an endpoint (see above); its scale is $h+1$.

Finally, if there is only one endpoint, its scale must be equal to $h+2$.

3) With each endpoint $v$ of scale $h_{v}=+1$ we associate one of the monomials in (2.16) contributing to $\mathcal{V}^{(0)}$ and a set $\mathbf{x}_{v}$ of space-time points (the corresponding integration variables); with each endpoint of scale $h_{v} \leq 0$ we associate a contribution of type $\lambda$, that is a contribution of the form (2.26), with $h=h_{v}-1$, and the corresponding set $\mathbf{x}_{v}$ of space-time points. We impose the constraint that, if $v$ is an endpoint, $h_{v}=h_{v^{\prime}}+1$, if $v^{\prime}$ is the non trivial vertex immediately preceding $v$.

Given a vertex $v$, which is not an endpoint, $\mathbf{x}_{v}$ will denote the family of all space-time points associated with one of the endpoints following $v$. 
4) If $v$ is not an endpoint, the cluster $L_{v}$ with scale $h_{v}$ is the set of endpoints following the vertex $v$; if $v$ is an endpoint, it is itself a (trivial) cluster. The tree provides an organization of endpoints into a hierarchy of clusters.

5) The trees containing only the root and an endpoint of scale $h+1$ (note that they do not belong to $\mathcal{T}_{h, 1}$ ) will be called the trivial trees.

6) We introduce a field label $f$ to distinguish the field variables appearing in the terms associated with the endpoints as in item 3); the set of field labels associated with the endpoint $v$ will be called $I_{v}$. Analogously, if $v$ is not an endpoint, we shall call $I_{v}$ the set of field labels associated with the endpoints following the vertex $v ; \mathbf{x}(f), \varepsilon(f)= \pm$ and $\sigma(f)=\uparrow \downarrow$ will denote the space-time point, the creation/annihilation index and the spin index, respectively, of the field variable with label $f$.

In terms of these trees, the effective potential $\mathcal{V}^{(h)}, h \leq 1$, can be written as

$$
\mathcal{V}^{(h)}\left(\psi^{(\leq h)}\right)+L \beta \tilde{F}_{h+1}=\sum_{n=1}^{\infty} \sum_{\tau \in \mathcal{T}_{h, n}} \mathcal{V}^{(h)}\left(\tau, \psi^{(\leq h)}\right),
$$

where, if $v_{0}$ is the first vertex of $\tau$ and $\tau_{1}, \ldots, \tau_{s}\left(s=s_{v_{0}}\right)$ are the subtrees of $\tau$ with root $v_{0}, \mathcal{V}^{(h)}\left(\tau, \psi^{(\leq h)}\right)$ is defined inductively by the relation

$$
\mathcal{V}^{(h)}\left(\tau, \psi^{(\leq h)}\right)=\frac{(-1)^{s+1}}{s !} \mathcal{E}_{h+1}^{T}\left[\overline{\mathcal{V}}^{(h+1)}\left(\tau_{1}, \psi^{(\leq h+1)}\right) ; \ldots ; \overline{\mathcal{V}}^{(h+1)}\left(\tau_{s}, \psi^{(\leq h+1)}\right)\right]
$$

and $\overline{\mathcal{V}}^{(h+1)}\left(\tau_{i}, \psi^{(\leq h+1)}\right)$

a) is equal to $\mathcal{R} \mathcal{V}^{(h+1)}\left(\tau_{i}, \psi^{(\leq h+1)}\right)$ if the subtree $\tau_{i}$ is not trivial;

b) if $\tau_{i}$ is trivial and $h<-1$, it is equal to $\mathcal{L}_{4} \mathcal{V}^{(h+1)}$ or, if $h=-1$, to one of the monomials contributing to $\mathcal{V}^{(0)}\left(\psi^{\leq 0}\right)$.

$\mathcal{E}_{h+1}^{T}$ denotes the truncated expectation with respect to the measure $P\left(d \psi^{(h+1)}\right)$, that is

$$
\left.\mathcal{E}_{h+1}^{T}\left(X_{1} ; \ldots ; X_{p}\right) \equiv \frac{\partial^{p}}{\partial \lambda_{1} \ldots \partial \lambda_{p}} \log \int \prod_{\omega} P\left(d \psi_{\omega}^{(h+1)}\right) e^{\lambda_{1} X_{1}+\ldots \lambda_{p} X_{p}}\right|_{\lambda_{i}=0} .
$$

This means, in particular, that, in (2.79), one has to use for the field variables the sector decomposition (2.56). The sector decomposition induces a further decomposition of the functions $\mathcal{V}^{(h)}\left(\tau, \psi^{(\leq h)}\right)$ in the r.h.s. of $(2.78)$ and in order to describe it we need some more definitions.

We associate with any vertex $v$ of the tree a subset $P_{v}$ of $I_{v}$, the external fields of $v$. These subsets must satisfy various constraints. First of all, if $v$ is not an endpoint and $v_{1}, \ldots, v_{s_{v}}$ are the vertices immediately following it, then $P_{v} \subset \cup_{i} P_{v_{i}}$; if $v$ is an endpoint, $P_{v}=I_{v}$. Given a vertex $v,\left|P_{v}\right|=2$ is not allowed and vertices $v$ with $\left|P_{v}\right|=4$ are necessarily endpoints. We shall denote $Q_{v_{i}}$ the intersection of $P_{v}$ and $P_{v_{i}}$; this definition implies that $P_{v}=\cup_{i} Q_{v_{i}}$. The subsets $P_{v_{i}} \backslash Q_{v_{i}}$, whose union $\mathcal{I}_{v}$ will be made, by definition, of the internal fields of $v$, have to be non 
empty, if $s_{v}>1$. Given $\tau \in \mathcal{T}_{h, n}$, there are many possible choices of the subsets $P_{v}$, $v \in \tau$, compatible with all the constraints. We shall denote $\mathcal{P}_{\tau}$ the family of all these choices and $\mathbf{P}$ the elements of $\mathcal{P}_{\tau}$.

Moreover, we associate with any $f \in \mathcal{I}_{v}$ a scale label $h(f)=h_{v}$ and an index $\omega(f) \in O_{h(f)}$. Note that $h(f)$ and $\omega(f)$ single out a sector of scale $h(f)$ and sector index $\omega(f)$ associated with the field variable of index $f$. In this way we assign $h(f)$ and $\omega(f)$ to each field label $f$, except those which correspond to the set $P_{v_{0}}$; we associate with any $f \in P_{v_{0}}$ the scale label $h(f)=h$ and a sector index $\omega(f) \in O_{h}$. We shall also put, for any $v \in \tau, \Omega_{v}=\left\{\omega(f), f \in P_{v}\right\}$. We shall call $\mathcal{O}_{\tau}$ the family of possible values of $\Omega=\left\{\omega(f), f \in \cup_{v} I_{v}\right\}$.

With these definitions, we can rewrite $\mathcal{V}^{(h)}\left(\tau, \psi^{(\leq h)}\right)$ in the r.h.s. of (2.78) as:

$$
\begin{aligned}
\mathcal{V}^{(h)}\left(\tau, \psi^{(\leq h)}\right) & =\sum_{\mathbf{P} \in \mathcal{P}_{\tau}, \Omega \in \mathcal{O}_{\tau}} \mathcal{V}^{(h)}(\tau, \mathbf{P}, \Omega), \\
\mathcal{V}^{(h)}(\tau, \mathbf{P}, \Omega) & =\int d \mathbf{x}_{v_{0}} \tilde{\psi}_{\Omega_{v_{0}}}^{(\leq h)}\left(P_{v_{0}}\right) K_{\tau, \mathbf{P}, \Omega}^{(h+1)}\left(\mathbf{x}_{v_{0}}\right),
\end{aligned}
$$

where

$$
\tilde{\psi}_{\Omega_{v}}^{(\leq h)}\left(P_{v}\right)=\prod_{f \in P_{v}} e^{i \varepsilon(f) \vec{p}_{F}^{(h)}\left(\theta_{h, \omega(f)}\right) \vec{x}(f)} \psi_{\mathbf{x}(f), \sigma(f), \omega(f)}^{(\leq h) \varepsilon(f)}
$$

and $K_{\tau, \mathbf{P}, \Omega}^{(h+1)}\left(\mathbf{x}_{v_{0}}\right)$ is defined inductively by the equation, valid for any $v \in \tau$ which is not an endpoint,

$$
K_{\tau, \mathbf{P}, \Omega}^{\left(h_{v}\right)}\left(\mathbf{x}_{v}\right)=\frac{1}{s_{v} !} \prod_{i=1}^{s_{v}}\left[K_{v_{i}}^{\left(h_{v}+1\right)}\left(\mathbf{x}_{v_{i}}\right)\right] \mathcal{E}_{h_{v}}^{T}\left[\tilde{\psi}_{\Omega_{1}}^{\left(h_{v}\right)}\left(P_{v_{1}} \backslash Q_{v_{1}}\right), \ldots, \tilde{\psi}_{\Omega_{s_{v}}}^{\left(h_{v}\right)}\left(P_{v_{s_{v}}} \backslash Q_{v_{s_{v}}}\right)\right]
$$

where $\Omega_{i}=\left\{\omega(f), f \in P_{v_{i}} \backslash Q_{v_{i}}\right\}$ and $\tilde{\psi}_{\Omega_{i}}^{\left(h_{v}\right)}\left(P_{v_{i}} \backslash Q_{v_{i}}\right)$ has a definition similar to (2.82). Moreover, if $v$ is an endpoint and $h_{v} \leq 0, K_{v}^{\left(h_{v}\right)}\left(\mathbf{x}_{v}\right)=\lambda_{h_{v}-1}\left(\mathbf{x}_{v}\right)$, while if $h_{v}=+1 K_{v}^{(1)}$ is equal to one of the kernels of the monomials in (2.16).

(2.78)-(2.81) is not the final form of our expansion; we further decompose $\mathcal{V}^{(h)}(\tau, \mathbf{P}, \Omega)$, by using the following representation of the truncated expectation in the r.h.s. of (2.83). Let us put $s=s_{v}, P_{i} \equiv P_{v_{i}} \backslash Q_{v_{i}}$; moreover we order in an arbitrary way the sets $P_{i}^{ \pm} \equiv\left\{f \in P_{i}, \varepsilon(f)= \pm\right\}$, we call $f_{i j}^{ \pm}$their elements and we define $\mathbf{x}^{(i)}=\cup_{f \in P_{i}^{-}} \mathbf{x}(f), \mathbf{y}^{(i)}=\cup_{f \in P_{i}^{+}} \mathbf{x}(f), \mathbf{x}_{i j}=\mathbf{x}\left(f_{i, j}^{-}\right), \mathbf{y}_{i j}=\mathbf{x}\left(f_{i, j}^{+}\right)$. Note that $\sum_{i=1}^{s}\left|P_{i}^{-}\right|=\sum_{i=1}^{s}\left|P_{i}^{+}\right| \equiv n$, otherwise the truncated expectation vanishes. A couple $l \equiv\left(f_{i j}^{-}, f_{i^{\prime} j^{\prime}}^{+}\right) \equiv\left(f_{l}^{-}, f_{l}^{+}\right)$will be called a line joining the fields with labels $f_{i j}^{-}, f_{i^{\prime} j^{\prime}}^{+}$, sector indices $\omega_{l}^{-}=\omega\left(f_{l}^{-}\right), \omega_{l}^{+}=\omega\left(f_{l}^{+}\right)$and spin indices $\sigma_{l}^{-}=\sigma\left(f_{l}^{-}\right)$, $\sigma_{l}^{+}=\sigma\left(f_{l}^{+}\right)$, connecting the points $\mathbf{x}_{l} \equiv \mathbf{x}_{i, j}$ and $\mathbf{y}_{l} \equiv \mathbf{y}_{i^{\prime} j^{\prime}}$, the endpoints of $l$. Moreover, if $\omega_{l}^{-}=\omega_{l}^{+}$, we shall put $\omega_{l} \equiv \omega_{l}^{-}=\omega_{l}^{+}$. Then, it is well known [Le, GM] that, up to a sign, if $s>1$, 


$$
\begin{aligned}
\mathcal{E}_{h}^{T}\left(\tilde{\psi}_{\Omega_{1}}^{(h)}\left(P_{1}\right), \ldots, \tilde{\psi}_{\Omega_{s}}^{(h)}\left(P_{s}\right)\right) & = \\
& \sum_{T} \prod_{l \in T} \tilde{g}_{\omega_{l}}^{(h)}\left(\mathbf{x}_{l}-\mathbf{y}_{l}\right) \delta_{\omega_{l}^{-}, \omega_{l}^{+}} \delta_{\sigma_{l}^{-}, \sigma_{l}^{+}} \int d P_{T}(\mathbf{t}) \operatorname{det} G^{h, T}(\mathbf{t})
\end{aligned}
$$

where

$$
\tilde{g}_{\omega}^{(h)}(\mathbf{x})=e^{-i \vec{p}_{F}\left(\theta_{h, \omega}\right) \vec{x}} g_{\omega}^{(h)}(\mathbf{x})
$$

and $T$ is a set of lines forming an anchored tree graph between the clusters of points $\mathbf{x}^{(i)} \cup \mathbf{y}^{(i)}$, that is $T$ is a set of lines, which becomes a tree graph if one identifies all the points in the same cluster. Moreover $\mathbf{t}=\left\{t_{i, i^{\prime}} \in[0,1], 1 \leq i, i^{\prime} \leq s\right\}, d P_{T}(\mathbf{t})$ is a probability measure with support on a set of $\mathbf{t}$ such that $t_{i, i^{\prime}}=\mathbf{u}_{i} \cdot \mathbf{u}_{i^{\prime}}$ for some family of vectors $\mathbf{u}_{i} \in \mathbb{R}^{s}$ of unit norm. Finally $G^{h, T}(\mathbf{t})$ is a $(n-s+1) \times(n-s+1)$ matrix, whose elements are given by

$$
G_{i j, i^{\prime} j^{\prime}}^{h, T}=t_{i, i^{\prime}} \tilde{g}_{\omega_{l}}^{(h)}\left(\mathbf{x}_{i j}-\mathbf{y}_{i^{\prime} j^{\prime}}\right) \delta_{\omega_{l}^{-}, \omega_{l}^{+}}
$$

with $\left(f_{i j}^{-}, f_{i^{\prime} j^{\prime}}^{+}\right)$not belonging to $T$.

In the following we shall use (2.84) even for $s=1$, when $T$ is empty, by interpreting the r.h.s. as equal to 1 , if $\left|P_{1}\right|=0$, otherwise as equal to $\operatorname{det} G^{h}=$ $\mathcal{E}_{h}^{T}\left(\tilde{\psi}^{(h)}\left(P_{1}\right)\right)$.

If we apply the expansion (2.84) in each non trivial vertex of $\tau$, we get an expression of the form

$$
\mathcal{V}^{(h)}(\tau, \mathbf{P}, \Omega)=\sum_{T \in \mathbf{T}} \int d \mathbf{x}_{v_{0}} \tilde{\psi}_{\Omega_{v_{0}}}^{(\leq h)}\left(P_{v_{0}}\right) W_{\tau, \mathbf{P}, \Omega \backslash \Omega_{v_{0}}, T}^{(h)}\left(\mathbf{x}_{v_{0}}\right) \equiv \sum_{T \in \mathbf{T}} \mathcal{V}^{(h)}(\tau, \mathbf{P}, \Omega, T),
$$

where $\mathbf{T}$ is a special family of graphs on the set of points $\mathbf{x}_{v_{0}}$, obtained by putting together an anchored tree graph $T_{v}$ for each non trivial vertex $v$. Note that any graph $T \in \mathbf{T}$ becomes a tree graph on $\mathbf{x}_{v_{0}}$, if one identifies all the points in the sets $x_{v}$, for any vertex $v$ which is also an endpoint. Given $\tau \in \mathcal{T}_{h, n}$ and the labels $\mathbf{P}, \Omega, T$, calling $v_{i}^{*}, \ldots, v_{n}^{*}$ the endpoints of $\tau$ and putting $h_{i}=h_{v_{i}^{*}}$, the explicit representation of $W_{\tau, \mathbf{P}, \Omega \backslash \Omega_{v_{0}}, T}^{(h)}\left(\mathbf{x}_{v_{0}}\right)$ in $(2.87)$ is

$$
\begin{aligned}
W_{\tau, \mathbf{P}, \Omega \backslash \Omega_{v_{0}}, T}\left(\mathbf{x}_{v_{0}}\right) & =\left[\prod_{i=1}^{n} K_{v_{i}^{*}}^{h_{i}}\left(\mathbf{x}_{v_{i}^{*}}\right)\right]\left\{\prod_{\substack{v \\
\text { not e. p. }}} \frac{1}{s_{v} !} \int d P_{T_{v}}\left(\mathbf{t}_{v}\right)\right. \\
\cdot & \left.\left.\operatorname{det} G^{h_{v}, T_{v}}\left(\mathbf{t}_{v}\right)\left[\prod_{l \in T_{v}} \delta_{\omega_{l}^{+}, \omega_{l}^{-}} \delta_{\sigma_{l}^{-}, \sigma_{l}^{+}} \tilde{g}_{\omega_{l}}^{\left(h_{v}\right)}\left(\mathbf{x}_{l}-\mathbf{y}_{l}\right)\right]\right]\right\} .
\end{aligned}
$$

\subsection{Modification of the running coupling functions}

Let us consider the expansion described in previous section and let us remark that, thanks to momentum conservation and compact support properties of propagator Fourier transforms, $\mathcal{V}^{(h)}(\tau, \mathbf{P}, \Omega)$ vanishes for some choices of $\Omega$. In the following bounds it will be crucial to take into account this constraint, and for this reason we introduce a different representation of the running coupling functions $\lambda_{h}$, in 
order to include in the new definitions the momentum constraints on the external lines of the corresponding vertices.

We define, for any $h \leq 0$ and $\omega \in O_{h}$, the s-sector $S_{h, \omega}$ as

$$
S_{h, \omega}=\left\{\vec{k}=\rho \vec{e}_{r}(\theta) \in \mathbb{R}^{2}:\left|\varepsilon_{h}(\vec{k})-\mu\right| \leq \gamma^{h} e_{0}, \zeta_{h, \omega}(\theta) \neq 0\right\} .
$$

Remark. Note that the definition of s-sector has the property, to be used extensively in the following, that the s-sector $S_{h+1, \omega}$ of scale $h+1$ contains the union of two s-sectors of scale $h: S_{h+1, \omega} \supseteq\left\{S_{h, 2 \omega} \cup S_{h, 2 \omega+1}\right\}$. This follows from the definition of $\zeta_{h, \omega}$ and the inductive assumption on $E_{h}(\mathbf{k})$, see (2.38), together with the fact that $\gamma=4$ (so that $\gamma^{1 / 2}=2$ ). Moreover $S_{h, 2 \omega}$ and $S_{h, 2 \omega+1}$ are the two only sectors on scale $h$ strictly contained into $S_{h+1, \omega}$.

We now observe that the field variables $\hat{\psi}_{\mathbf{k}(f), \omega(f), \sigma(f)}^{\leq h_{v_{0}}, \varepsilon(f)}$ have the same supports as the functions $C_{h_{v_{0}}}^{-1}(\mathbf{k}(f)) \zeta_{h_{v_{0}}, \omega(f)}(\theta(f))$ and $h(f) \leq h_{i}-1, \forall f \in P_{v_{i}^{*}}$; hence in the expression $(2.88)$, we can freely multiply $\hat{K}_{v_{i}^{*}}^{h_{i}}\left(\mathbf{k}_{v_{i}^{*}}\right)$ by $\prod_{f \in P_{v_{i}^{*}}} \tilde{F}_{h_{i}-1, \tilde{\omega}(f)}(\vec{k})$, where $\tilde{F}_{h, \omega}(\vec{k})$ is a smooth function equal to 1 on $S_{h, \omega}$ and with a support slightly greater than $S_{h, \omega}$, while $\tilde{\omega}(f) \in O_{h_{i}-1}$ is the unique sector index such that $S_{h(f), \omega(f)} \subseteq S_{h_{i}-1, \tilde{\omega}(f)}$. In order to formalize this statement, it is useful to introduce the following definition.

Let $G(\underline{\vec{x}})$ be a function of $2 p$ variables $\underline{\vec{x}}=\left(\vec{x}_{1}, \ldots, \vec{x}_{2 p}\right)$ with Fourier transform $\hat{G}(\underline{\vec{k}})$, defined so that $G(\underline{\vec{x}})=\int d \underline{\vec{k}}(2 \pi)^{-4 p} \exp \left(-i \sum_{l=1}^{2 p} \varepsilon_{i} \vec{k}_{i} \vec{x}_{i}\right) \hat{G}(\underline{\vec{k}})$, where $\varepsilon_{1}, \ldots, \varepsilon_{p}=-\varepsilon_{p+1}=\cdots=-\varepsilon_{2 p}=+1$. Then, we define, given $h \leq 0$ and a family $\underline{\omega}=\left\{\omega_{i} \in O_{h}, i=1, \ldots, 2 p\right\}$ of sector indices,

$$
\left(\mathfrak{F}_{2 p, h, \underline{\omega}} * G\right)(\underline{\vec{x}})=\int \frac{d \underline{\vec{k}}}{(2 \pi)^{4 p}} e^{-i \sum_{l=1}^{2 p} \varepsilon_{i} \vec{k}_{i} \vec{x}_{i}}\left[\prod_{i=1}^{2 p} \tilde{F}_{h, \omega_{i}}\left(\vec{k}_{i}\right)\right] \hat{G}(\underline{\vec{k}}) .
$$

Hence, if we put $p_{i}=\left|P_{v_{i}^{*}}\right|, \Omega_{v_{i}^{*}}=\left\{\omega(f), f \in P_{v_{i}^{*}}\right\}$ and we define

$$
\tilde{K}_{v_{i}^{*}, \Omega_{v_{i}^{*}}}^{h_{i}}\left(\mathbf{x}_{v_{i}^{*}}\right)=\left(\mathfrak{F}_{p_{i}, h_{i}-1, \Omega_{v_{i}^{*}}} * K_{v_{i}^{*}}^{h_{i}}\right)\left(\mathbf{x}_{v_{i}^{*}}\right),
$$

we can substitute in $(2.88)$ each $K_{v_{i}^{*}}^{h_{i}}\left(\mathbf{x}_{v_{i}^{*}}\right)$ with $\tilde{K}_{v_{i}^{*}, \Omega_{v_{i}^{*}}}^{h_{i}}\left(\mathbf{x}_{v_{i}^{*}}\right)$, to be called the $\bmod$ ified coupling functions. In particular, if $v_{i}^{*}$ is of type $\lambda$, we shall denote the corresponding modified coupling functions by the symbol $\tilde{\lambda}_{h_{i}-1, \Omega_{v_{i}^{*}}}\left(\mathbf{x}_{v_{i}^{*}}\right)$. The smallness condition on $\lambda$ can be now stated in terms of $\tilde{\lambda}_{h_{i}-1, \Omega_{v_{i}^{*}}}\left(\mathbf{x}_{v_{i}^{*}}\right)$ as follows:

$$
\frac{1}{L^{2} \beta} \int d \mathbf{x}_{v_{i}^{*}}\left|\tilde{\lambda}_{h_{i}-1, \Omega_{v_{i}^{*}}}\left(\mathbf{x}_{v_{i}^{*}}\right)\right| \leq C|U|,
$$

for some constant $C>0$.

We shall call $W_{\tau, \mathbf{P}, \Omega, T}^{(\bmod )}\left(\mathbf{x}_{v_{0}}\right)$ the expression we get from $W_{\tau, \mathbf{P}, \Omega \backslash \Omega_{v_{0}}, T}\left(\mathbf{x}_{v_{0}}\right)$ by the substitution of the kernels $K_{v_{i}^{*}}^{h_{i}}\left(\mathbf{x}_{v_{i}^{*}}\right)$ with the modified ones. Note that 
$W_{\tau, \mathbf{P}, \Omega, T}^{(\text {mod })}\left(\mathbf{x}_{v_{0}}\right)$ is not independent of $\Omega_{v_{0}}$, unlike $W_{\tau, \mathbf{P}, \Omega \backslash \Omega_{v_{0}}, T}\left(\mathbf{x}_{v_{0}}\right)$, and that $W_{\tau, \mathbf{P}, \Omega, T}^{\text {(mod) }}\left(\mathbf{x}_{v_{0}}\right)$ is equal to $W_{\tau, \mathbf{P}, \Omega \backslash \Omega_{v_{0}, T}}\left(\mathbf{x}_{v_{0}}\right)$, only if $\left|P_{v_{0}}\right|=0$; however, the previous considerations imply that, if $p_{0}=\left|P_{v_{0}}\right|>0$,

$$
\left(\mathfrak{F}_{p_{0}, h, \Omega_{v_{0}}} * W_{\tau, \mathbf{P}, \Omega, T}^{(\bmod )}\right)\left(\mathbf{x}_{v_{0}}\right)=\left(\mathfrak{F}_{p_{0}, h, \Omega_{v_{0}}} * W_{\tau, \mathbf{P}, \Omega \backslash \Omega_{v_{0}}, T}\right)\left(\mathbf{x}_{v_{0}}\right),
$$

a trivial remark which will be important in the discussion of the running coupling functions flow in next chapter.

\subsection{Bounds for the effective potentials and the free energy}

In order to explicitly keep track of the constraints satisfied by the sector indices $\Omega$, which is crucial for performing the dimensional bounds on the free energy, we introduce the following constraint functions. Given a tree $\tau \in \mathcal{T}_{h, n}$ with all its labels, a vertex $v \in \tau$ and the set of anisotropic sector indices $\Omega_{v}=\{\omega(f) \in$ $\left.O_{h(f)}, f \in P_{v}\right\}$ labelled by $P_{v}$, we define

$$
\chi\left(\Omega_{v}\right)= \begin{cases}\mathbb{1}\left(\forall f \in P_{v}, \exists \vec{k}(f) \in S_{h(f), \omega(f)}: \sum_{f \in P_{v}} \varepsilon(f) \vec{k}(f)=0\right), & \left|P_{v}\right| \leq 8 \\ 1, & \left|P_{v}\right| \geq 10 .\end{cases}
$$

The previous considerations and (2.35) imply that

$$
F_{L, \beta} \leq F_{0}+\sum_{h=h_{\beta}}^{0}\left[t_{h}+\sum_{n=1}^{\infty} \sum_{\tau \in \mathcal{T}_{h, n}} \sum_{\substack{\mathbf{P} \in \mathcal{P}_{\tau} \\\left|P_{v_{0}}\right|=0}} \sum_{T \in \mathbf{T}} J_{h, n}^{(0)}(\tau, \mathbf{P}, T)\right],
$$

with

$$
J_{h, n}^{(F)}(\tau, \mathbf{P}, T)=\sum_{\Omega \backslash \Omega_{\mathrm{ext}}^{(F)}}\left[\prod_{v} \chi\left(\Omega_{v}\right)\right] \int d\left(\mathbf{x}_{v_{0}} \backslash \mathbf{x}^{*}\right)\left|W_{\tau, \mathbf{P}, \Omega, T}^{(\bmod )}\left(\mathbf{x}_{v_{0}}\right)\right|,
$$

where $\mathbf{x}^{*}$ is an arbitrary point in $\mathbf{x}_{v_{0}}, \Omega \in \mathcal{O}_{\tau}$ and, if $2 l_{0}=\left|P_{v_{0}}\right|>0$ and $0<F \leq 2 l_{0}, \Omega_{\text {ext }}^{(F)} \subset \Omega_{v_{0}}$ is an arbitrary subset of the sector indices in $\Omega_{v_{0}}$ of cardinality $F,\left|\Omega_{\text {ext }}^{(F)}\right|=F$ : in particular, if $l_{0}=0$ or $F=0, \sum_{\Omega \backslash \Omega_{\text {ext }}^{(F)} \in \mathcal{O}_{\tau}}$ coincides with $\sum_{\Omega \in \mathcal{O}_{\tau}}$. Note that we could freely insert $\prod_{v} \chi\left(\Omega_{v}\right)$ in $(2.96)$, because of the constraints following from momentum conservation and the compact support properties of propagator Fourier transforms; here we used that umklapp processes are impossible if $\left|P_{v}\right| \leq 8$ (because of the condition on $\mu$ chosen in Theorem 1.1); for vertices with $\left|P_{v}\right| \geq 10$ we discarded any possible constraint coming from momentum conservation (modulo $2 \pi \mathbb{Z}^{2}$ ).

The following theorem will be the starting point of our analysis.

Theorem 2.1. Given $h_{\beta} \leq h \leq 0, \tau \in \mathcal{T}_{h, n}, \mathbf{P} \in \mathcal{P}_{\tau}, T \in \mathbf{T}$, if $E_{j}(\mathbf{k})$ satisfies

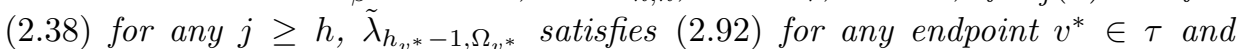
$U_{0}\left|h_{\beta}\right|=c_{0}$ is small enough, then 


$$
\begin{aligned}
& J_{h, n}^{(0)}(\tau, \mathbf{P}, T) \leq(c|U|)^{n} \gamma^{h\left(2-\frac{3}{4}\left|P_{v_{0}}\right|\right)} \prod_{v \text { not e. p. }} \frac{1}{s_{v} !} \gamma^{\delta\left(\left|P_{v}\right|\right)}, \\
& J_{h, n}^{(1)}(\tau, \mathbf{P}, T) \leq(c|U|)^{n} \gamma^{h\left(\frac{5}{2}-\frac{3}{4}\left|P_{v_{0}}\right|\right)} \prod_{v \text { not e. p. }} \frac{1}{s_{v} !} \gamma^{\delta\left(\left|P_{v}\right|\right)}
\end{aligned}
$$

where

$$
\delta(p)=1-\frac{p}{4}+\mathbb{1}(p \geq 10) .
$$

Remark. By construction, clusters with $\left|P_{v}\right|=2$ are not allowed, and clusters with $\left|P_{v}\right|=4$ are necessarily endpoints. Then the exponent $\delta\left(\left|P_{v}\right|\right)$ appearing in (2.97) is always $\leq-1 / 2$ and, as a consequence, it can be easily proved that the sums over $\mathbf{P}$ and $\tau$ in (2.95) converge exponentially, see [BGM], so that the sum in the r.h.s. of (2.95) is absolutely convergent and, under the assumptions of the theorem, $\lim _{L \rightarrow \infty} F_{L, \beta}$ does exist and is $O(U)$. This proves the first claim in the Remark following Theorem 1.1.

In the remaining part of this section we shall give the proof of Theorem 2.1, already presented in Section 2.7 of [BGM] (see in particular eq. (2.101)) in a slightly different case and with a smallness condition on the $\tilde{\lambda}_{h}$ different from (2.92) (but dimensionally equivalent). Moreover, the proof will be presented here in a form convenient for performing the improved dimensional bounds on $E_{h}(\mathbf{k})$ in next chapter.

Proof. We shall describe the proof in the case that all the endpoints are of type $\lambda$. A posteriori, it will be clear that the possible presence of endpoints of scale +1 with $p \geq 6$ external legs (produced by the ultraviolet integration, see Appendix A) does not qualitatively change the argument.

We begin with considering the case $F=1$. By using the definition of $W_{\tau, \mathbf{P}, \Omega, T}^{(\bmod )}\left(\mathbf{x}_{v_{0}}\right)$ and the expression (2.88), we can bound the r.h.s. of (2.96) as

$$
\begin{aligned}
\sum_{\Omega \backslash \Omega_{\mathrm{ext}}^{(1)}}\left[\prod_{v} \chi\left(\Omega_{v}\right)\right] & \int \prod_{l \in T^{*}} d\left(\mathbf{x}_{l}-\mathbf{y}_{l}\right)\left[\prod_{i=1}^{n}\left|\widetilde{\lambda}_{h_{i}-1, \Omega_{v_{i}^{*}}}\left(\mathbf{x}_{v_{i}^{*}}\right)\right|\right] \\
\cdot & \left\{\prod_{\substack{v \\
\text { not e. p. }}} \frac{1}{s_{v} !} \max _{\mathbf{t}_{v}}\left|\operatorname{det} G^{h_{v}, T_{v}}\left(\mathbf{t}_{v}\right)\right| \prod_{l \in T_{v}}\left|g_{\omega_{l}}^{\left(h_{v}\right)}\left(\mathbf{x}_{l}-\mathbf{y}_{l}\right)\right|\right\}
\end{aligned}
$$

where $T^{*}$ is a tree graph obtained from $T=\cup_{v} T_{v}$, by adding in a suitable (obvious) way, for each endpoint $v_{i}^{*}, i=1, \ldots, n$, two lines connecting the four space-time points belonging to $\mathbf{x}_{v_{i}^{*}}$.

A standard application of Gram-Hadamard inequality, combined with the dimensional bound on $g_{\omega}^{(h)}$ given by Lemma 2.2, implies (see eq. (2.80) of [BGM] and related proof) that

$$
\left|\operatorname{det} G_{\alpha}^{h_{v}, T_{v}}\left(\mathbf{t}_{v}\right)\right| \leq c^{\sum_{i=1}^{s_{v}}\left|P_{v_{i}}\right|-\left|P_{v}\right|-2\left(s_{v}-1\right)} \cdot \gamma^{h_{v} \frac{3}{4}\left(\sum_{i=1}^{s_{v}}\left|P_{v_{i}}\right|-\left|P_{v}\right|-2\left(s_{v}-1\right)\right)} .
$$


By using the bound (2.61) with $j=0$, it also follows that

$$
\prod_{v \text { not e. p. }} \frac{1}{s_{v} !} \int \prod_{l \in T_{v}} d\left(\mathbf{x}_{l}-\mathbf{y}_{l}\right)\left|g_{\omega_{l}}^{\left(h_{v}\right)}\left(\mathbf{x}_{l}-\mathbf{y}_{l}\right)\right| \leq c^{n} \prod_{v \text { not e. p. }} \frac{1}{s_{v} !} \gamma^{-h_{v}\left(s_{v}-1\right)} \text {. }
$$

The smallness assumption (2.92) on the size of $\tilde{\lambda}_{h_{v}-1, \Omega_{v}}$ implies that

$$
\int \prod_{l \in T^{*} \backslash \cup_{v} T_{v}} d\left(\mathbf{x}_{l}-\mathbf{y}_{l}\right) \prod_{i=1}^{n}\left|\widetilde{\lambda}_{h_{i}-1, \Omega_{v_{i}^{*}}}\left(\mathbf{x}_{v_{i}^{*}}\right)\right| \leq(C|U|)^{n}
$$

Finally, as we shall explain below, by suitably taking into account the constraint functions $\chi\left(\mathcal{S}\left(P_{v}\right)\right)$, the sum over the choices of the sector indices gives

$$
\begin{aligned}
& \sum_{\Omega \backslash \Omega_{\mathrm{ext}}^{(1)}} \prod_{v \in \tau}\left(\chi\left(\Omega_{v}\right) \prod_{l \in T_{v}} \delta_{\omega_{l}^{+}, \omega_{l}^{-}}\right) \leq \\
& c^{n} \gamma^{-\frac{1}{2} h n} \prod_{v \text { not e. p. }} \gamma^{\left[-\frac{1}{2} m_{4}(v)+\frac{1}{2}\left(\left|P_{v}\right|-3\right) \mathbb{1}\left(4 \leq\left|P_{v}\right| \leq 8\right)+\frac{1}{2}\left(\left|P_{v}\right|-1\right) \mathbb{1}\left(\left|P_{v}\right| \geq 10\right)\right],}
\end{aligned}
$$

where $m_{4}(v)$ denotes the number of endpoints (all of type $\lambda$, by hypothesis) following $v$ on $\tau$. It is straightforward to check that Theorem 2.1 in the case $F=1$ follows by combining the bounds (2.100), (2.101), (2.102) and (2.103).

If $F=0$ and $\left|P_{v_{0}}\right|>0$, the theorem simply follows by the remark that the r.h.s. of $(2.96)$, that is of the form $\sum_{\Omega}[\cdots]$ can be rewritten as $\sum_{\omega_{1} \in O_{h}} \sum_{\Omega \backslash \omega_{1}}[\cdots]$, where $\omega_{1}$ is an arbitrary sector index in $\Omega_{v_{0}}$. Now, the sum $\sum_{\Omega \backslash \omega_{1}}[\cdots]$ can be bounded as in the second line of (2.97), the first sum $\sum_{\omega_{1} \in O_{h}}$ gives a contribution $O\left(\gamma^{-h / 2}\right)$ and the first line of (2.97) follows. If $\left|P_{v_{0}}\right|=0$, the proof of (2.103) shows that the same bound is true if the sector $\Omega_{\text {ext }}^{(1)}$ is substituted with a suitable internal sector; hence the theorem follows by the same remark as before.

Let us now describe the proof of (2.103), that will be the starting point of the proof of the improved dimensional bounds on $E_{h}(\mathbf{k})$, to be described in next chapter.

Let us first note that, by the definition of $s$-sector $S_{h, \omega}$ and by the properties of $E_{h}(\mathbf{k})$, the following crucial property is true:

given $\omega \in O_{h}$ and the s-sector $S_{h, \omega}$, for any $j>h$ there is a unique $\omega^{\prime}(j ; \omega) \in O_{j}$ such that $S_{j, \omega^{\prime}(j ; \omega)} \supset S_{h, \omega}$.

This property allows us to give a meaning to the following definition. Given $\tau \in \mathcal{T}_{h, n}, \mathbf{P} \in \mathcal{P}_{\tau}$ and $v \in \tau$, we introduce the symbol $\Omega_{v}^{(j)}$ to denote the set

$$
\begin{aligned}
\Omega_{v}^{(j)} & =\left\{\omega(f), f \in P_{v}: h(f) \geq j\right\} \cup\left\{\omega^{\prime}(j ; \omega(f)), f \in P_{v}: j>h(f)\right\} \equiv \\
& \equiv\left\{\omega_{f}^{(j)} \in O_{j_{f}^{(j)}}, f \in P_{v}\right\},
\end{aligned}
$$


where the last identity defines the scales $j_{f}^{(j)}$ and the sector indices $\omega_{f}^{(j)}, f \in P_{v}$. The definition (2.104) implies in particular that $\Omega_{v}=\Omega_{v}^{(h)}$. With reference to definition (2.104), in the following we shall also denote:

$$
\chi\left(\Omega_{v}^{(j)}\right)=\mathbb{1}\left(\forall f \in P_{f}, \exists \vec{k}(f) \in S_{j_{f}^{(j)}, \omega_{f}^{(j)}}: \sum_{f \in P_{f}} \varepsilon(f) \vec{k}(f)=\overrightarrow{0}\right) .
$$

Note in particular that:

$$
\chi\left(\Omega_{v}^{(j)}\right) \leq \chi\left(\Omega_{v}^{(k)}\right), \quad \text { if } j \leq k .
$$

Given $\tau \in \mathcal{T}_{h, n}$, we define the set $V_{c}(\tau)$ of $c$-vertices of $\tau$ as the set of vertices $v$ of $\tau$ which either are endpoints or have the property that their set $\mathcal{I}_{v}$ of internal lines is non empty ${ }^{3}$; in the following we shall often drop the dependence on $\tau$ (when it is clear from the context). Note that by definition, if $\tau \in \mathcal{T}_{h, n}$, then $\left|V_{c}(\tau)\right|=O(n)$ and it holds $\prod_{v \in \tau} \chi\left(\Omega_{v}\right)=\prod_{v \in V_{c}} \chi\left(\Omega_{v}\right)$. Moreover, using that $\Omega_{v}=\Omega_{v}^{(h)}$, we see that we can replace the product $\prod_{v \in \tau} \chi\left(\Omega_{v}\right)$ in the 1.h.s. of (2.103) by $\prod_{v \in V_{c}} \chi\left(\Omega_{v}^{(h)}\right)$.

We now begin to inductively bound the l.h.s. of (2.103); first of all we shall bound the sum corresponding to the first $c$-vertex following the root; then we will iteratively enter its structure. After each step we will be left with a product of sector sums of the same form of the initial one, but on larger scales.

We call $\tilde{v}_{0}$ the first $c$-vertex following the root and $h_{0} \equiv h_{\tilde{v}_{0}}$ (the scale label of the legs belonging to $T_{\tilde{v}_{0}}$ ); using $(2.106)$, we find

$$
\prod_{v \in V_{c}} \chi\left(\Omega_{v}^{(h)}\right) \leq \chi\left(\Omega_{\tilde{v}_{0}}^{(h)}\right) \prod_{v>\tilde{v}_{0}, v \in V_{c}} \chi\left(\Omega_{v}^{\left(h_{0}\right)}\right) .
$$

Substituting (2.107) into the 1.h.s. of (2.103), we find

$$
\begin{aligned}
\sum_{\Omega \backslash \Omega_{\mathrm{ext}}^{(1)}} \prod_{v \in V_{c}}\left(\chi\left(\Omega_{v}^{(h)}\right) \prod_{l \in T_{v}} \delta_{\omega_{l}^{+}, \omega_{l}^{-}}\right) \leq \\
\quad \sum_{\Omega_{\tilde{v}_{0}}^{\left(h_{0}\right)}}^{*}\left[\sum_{\Omega_{\tilde{v}_{0}}^{(h)} \prec \Omega_{\tilde{v}_{0}}^{\left(h_{0}\right)}}^{*} \chi\left(\Omega_{\tilde{v}_{0}}^{(h)}\right)\right] \sum_{\Omega \backslash \Omega_{\tilde{v}_{0}}} \prod_{v>\tilde{v}_{0}, v \in V_{c}} \chi\left(\Omega_{v}^{\left(h_{0}\right)}\right) \prod_{l \in T} \delta_{\omega_{l}^{+}, \omega_{l}^{-}},
\end{aligned}
$$

where $\Omega_{\tilde{v}_{0}}^{(h)} \prec \Omega_{\tilde{v}_{0}}^{\left(h_{0}\right)}$ means that the indices in $\Omega_{\tilde{v}_{0}}^{(h)}$ satisfy the following constraint: given $f \in P_{v}$ and the corresponding index $\omega_{f}^{\left(h_{0}\right)} \in O_{j_{f}^{\left(h_{0}\right)}}$ of $\Omega_{\tilde{v}_{0}}^{\left(h_{0}\right)}$, then the index $\omega_{f}^{(h)} \in O_{j_{f}^{(h)}}$ of $\Omega_{\tilde{v}_{0}}^{(h)}$ is such that $S_{j_{f}^{(h)}, \omega_{f}^{(h)}} \subset S_{j_{f}^{\left(h_{0}\right)}, \omega_{f}^{\left(h_{0}\right)}}$. The symbol $*$ on the sums in the second line means that the sector index in $\Omega_{\text {ext }}^{(1)}$, associated to one of the fields in $P_{\tilde{v}_{0}}$ (say to the field $f_{0} \in P_{\tilde{v}_{0}}$ ), is not summed over.

\footnotetext{
${ }^{3} \mathrm{With}$ reference to the definition of $\chi$-vertices introduced in Sect. 3.1 of [BGM], a vertex is called a $c$-vertex if it is either an endpoint or a $\chi$-vertex. The prefix $c$-recalls that a constraint function $\chi$ is associated to any $c$-vertex.
} 
By the sector counting Lemma (see Lemma C.1 in Appendix C), the sum in square brackets can be bounded, uniformly in $\Omega_{\tilde{v}_{0}}^{\left(h_{0}\right)}$, as

$$
\sum_{\Omega_{\tilde{v}_{0}}^{(h)} \prec \Omega_{\tilde{v}_{0}}^{\left(h_{0}\right)}}^{*} \chi\left(\Omega_{\tilde{v}_{0}}^{(h)}\right) \leq c \gamma^{\left(h_{0}-h\right)\left[\frac{1}{2}\left(\left|P_{\tilde{v}_{0}}\right|-3\right) \mathbb{1}\left(4 \leq\left|P_{\tilde{v}_{0}}\right| \leq 8\right)+\frac{1}{2}\left(\left|P_{\tilde{v}_{0}}\right|-1\right) \mathbb{1}\left(\left|P_{\tilde{v}_{0}}\right| \geq 10\right)\right]}
$$

so that the r.h.s. of (2.108) can be bounded by the r.h.s. of (2.109) times

$$
\sum_{\cup_{v} \Omega_{v}^{\left(h_{0}\right)}}^{*}\left[\prod_{v>\tilde{v}_{0}, v \in V_{c}} \chi\left(\Omega_{v}^{\left(h_{0}\right)}\right)\right]\left[\prod_{l \in T} \delta_{\omega_{l}^{+}, \omega_{l}^{-}}\right],
$$

where the $*$ on the sum recalls again that we are not summing over the sector index of $f_{0} \in P_{\tilde{v}_{0}}$.

We will now prove that $(2.110)$ can be reduced to a product of contributions analogue to the l.h.s. of (2.108), with $h_{0}$ replacing $h$. In fact, calling $\underline{\tilde{v}}_{0}=\left\{v_{1}, \ldots, v_{\tilde{v}_{0}}\right\}$ the set of $c$-vertices immediately following $\tilde{v}_{0}$ on $\tau$ and $\Omega_{\underline{\underline{v}}_{0}}^{\left(h_{0}\right)}=$ $\cup_{v \in \underline{\tilde{v}}_{0}} \Omega_{v}^{\left(h_{0}\right)}$, we can rewrite $(2.110)$ as

$$
\begin{gathered}
\sum_{\Omega_{\underline{\underline{v}}_{0}}^{\left(h_{0}\right)}}^{*} \prod_{v \in \underline{\tilde{v}}_{0}}\left[\sum_{\cup_{w>v} \Omega_{w}^{\left(h_{0}\right)} \backslash \Omega_{v}^{\left(h_{0}\right)}} \prod_{w \geq v, w \in V_{c}} \chi\left(\Omega_{w}^{\left(h_{0}\right)}\right) \prod_{l \in \cup_{w \geq v} T_{w}} \delta_{\omega_{l}^{+}, \omega_{l}^{-}}\right] \\
\cdot \prod_{l \in T_{\tilde{v}_{0}}} \delta_{\omega_{l}^{+}, \omega_{l}^{-}} \equiv \sum_{\Omega_{\tilde{v}_{0}}^{\left(h_{0}\right)}}^{*} \prod_{v \in \tilde{v}_{0}} F_{v}\left(\Omega_{v}^{\left(h_{0}\right)}\right) \prod_{l \in T_{\tilde{v}_{0}}} \delta_{\omega_{l}^{+}, \omega_{l}^{-}} .
\end{gathered}
$$

The function $F_{v}\left(\Omega_{v}^{\left(h_{0}\right)}\right)$, defined by $(2.111)$, is the sum over the "internal sector indices" of the product of the constraint functions corresponding to the vertex $v$. Note that also the l.h.s. of (2.108) could have been written in terms of one of these functions; the l.h.s. of (2.108) is in fact equal to $\sum_{\Omega_{v_{0}}^{(h)}}^{*} F_{v_{0}}\left(\Omega_{v_{0}}^{(h)}\right)$.

We now choose as the root of $T_{\tilde{v}_{0}}$ the vertex $v_{i} \in \underline{\tilde{v}}_{0}$ such that $f_{0} \in P_{v_{i}}$; then we select a leaf $v^{*}$ of $T_{\tilde{v}_{0}}$ and we call $l^{*}$ the branch of $T_{\tilde{v}_{0}}$ anchored to $v^{*}$. Calling $\Omega_{\underline{\underline{v}}_{0} \backslash v^{*}}^{\left(h_{0}\right)}=\cup_{v \in \underline{\tilde{v}}_{0} \backslash v^{*}} \Omega_{v}^{\left(h_{0}\right)}$, we denote by $\mathcal{F}_{\underline{\tilde{v}}_{0} \backslash v^{*}}\left(\Omega_{\underline{\tilde{v}}_{0} \backslash v^{*}}\right)$ the product of the constraint functions corresponding to the set of vertices $\underline{\tilde{v}}_{0} \backslash v^{*}$ :

$$
\mathcal{F}_{\underline{\tilde{v}}_{0} \backslash v^{*}}\left(\Omega_{\underline{\underline{v}}_{0} \backslash v^{*}}^{\left(h_{0}\right)} \stackrel{\text { def }}{=} \prod_{v \in \underline{\underline{v}}_{0} \backslash v^{*}} F_{v}\left(\Omega_{v}^{\left(h_{0}\right)}\right) \prod_{l \in T_{\tilde{v}_{0}} \backslash l^{*}} \delta_{\omega_{l}^{+}, \omega_{l}^{-}}\right.
$$

so that we can rewrite $(2.111)$ as

$$
\sum_{\omega_{l^{*}}^{+}, \omega_{l^{*}}^{-}} \delta_{\omega_{l^{*}}^{+}, \omega_{l^{*}}^{-}} \sum_{\Omega_{\underline{\underline{v}}_{0} \backslash v^{*}}^{* *}}^{*} \mathcal{F}_{\underline{\tilde{v}}_{0} \backslash v^{*}}\left(\Omega_{\underline{\underline{v}}_{0} \backslash v^{*}}^{\left(h_{0}\right)}\right) \sum_{\Omega_{v^{*}}^{\left(h_{0}\right)}}^{*} F_{v^{*}}\left(\Omega_{v^{*}}^{\left(h_{0}\right)}\right)
$$

where the $* *$ on the second sum means that we are not summing neither on $\omega_{f_{0}}$ nor on $\omega_{l^{*}}$ and the $*$ on the third sum recalls that we are not summing over $\omega_{l^{*}}$. 
Bounding the last sum by $\sup _{\omega_{l^{*}}} \sum_{\Omega_{v^{*}}^{\left(h_{0}\right)}}^{*} F_{v^{*}}\left(\Omega_{v^{*}}^{\left(h_{0}\right)}\right)$, we see that the last sum can be factorized out:

$$
(2.113) \leq \sum_{\Omega_{\underline{\underline{v}}_{0} \backslash v^{*}}}^{*} \mathcal{F}_{\underline{\tilde{v}}_{0} \backslash v^{*}}\left(\Omega_{\underline{\tilde{v}}_{0} \backslash v^{*}}^{\left(h_{0}\right)}\right) \cdot \sup _{\omega_{l^{*}}}\left[\sum_{\Omega_{v^{*}}^{\left(h_{0}\right)}}^{*} F_{v^{*}}\left(\Omega_{v^{*}}^{\left(h_{0}\right)}\right)\right] .
$$

It is now clear that we can iterate the same procedure by choosing another leaf of $T_{\tilde{v}_{0}} \backslash l^{*}$ and by factorizing out the corresponding contribution. At the end of the procedure we reach the root of $T_{\tilde{v}_{0}}$ and we finally find that $(2.110)$ can be bounded by

$$
\prod_{v \in \underline{\tilde{v}}_{0}} \sup _{\omega_{v}^{*}} \sum_{\Omega_{v}^{\left(h_{0}\right)}}^{*} F_{v}\left(\Omega_{v}^{\left(h_{0}\right)}\right)
$$

where $\omega_{v}^{*}$ is the sector index corresponding to the line of $T_{\tilde{v}_{0}}$ entering $v \in \underline{\tilde{v}}_{0}$, if $v$ is not the root, or to $l_{0}$, otherwise; the $*$ on the sum $\sum_{\Omega_{v}^{\left(h_{0}\right)}}^{*}$ means that we are not summing over $\omega_{v}^{*}$.

Now, if $v \in \underline{\tilde{v}}_{0}$ is an endpoint, then the corresponding contribution in (2.115) can be easily bounded by

$$
\sum_{\Omega_{v}^{\left(h_{0}\right)}}^{*} F_{v}\left(\Omega_{v}^{\left(h_{0}\right)}\right) \leq c \gamma^{-\frac{h_{0}}{2}}
$$

where we used again the sector counting Lemma C.1.

If $v \in \underline{\tilde{v}}_{0}$ is not an endpoint, the corresponding factor in (2.115) has exactly the same form as the l.h.s. of (2.108), and we can bound it by repeating the same procedure described above; then, proceeding by induction, we find (2.103).

Before concluding this section, let us state a generalization of the bound (2.103), proved in Appendix D, that will be useful in the following.

Lemma 2.6. Given $h_{\beta} \leq h \leq 0$ and a tree $\tau \in \mathcal{T}_{h, n}$ with all its labels, let us consider the sum

$$
\sum_{\Omega \backslash \Omega_{\mathrm{ext}}^{(F)}} \prod_{v \in \tau}\left(\chi\left(\Omega_{v}\right) \prod_{l \in T_{v}} \delta_{\omega_{l}^{+}, \omega_{l}^{-}}\right)
$$

with $F$ compatible with $\mathbf{P}$, i.e., $F \leq\left|P_{v_{0}}\right|$. If $F=3$, (2.117) can be bounded by the r.h.s. of (2.103) times $\gamma^{\frac{h}{2}}$ and, if $F=5$, (2.117) can be bounded by the r.h.s. of (2.103) times $\gamma^{h}$.

The same result is true if $\tau$ is a trivial tree or $\tau \in \mathcal{T}_{h, 1}$ and $\left|P_{v_{0}}\right|=4$.

Lemma 2.6 allows to get the following generalization of Theorem 2.1.

Lemma 2.7. Given $h_{\beta} \leq h \leq 0, \tau \in \mathcal{T}_{h, n}, \mathbf{P} \in \mathcal{P}_{\tau}, T \in \mathbf{T}$, if $E_{k}(\mathbf{k})$ satisfies

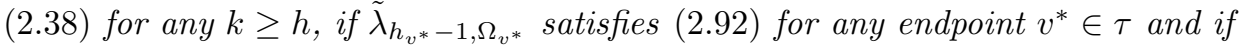


$U_{0}\left|h_{\beta}\right|=c_{0}$ is small enough, then

$$
\begin{aligned}
& J_{h, n}^{(3)}(\tau, \mathbf{P}, T) \leq(c|U|)^{n} \gamma^{h\left(3-\frac{3}{4}\left|P_{v_{0}}\right|\right)} \prod_{v \text { not e.p. }} \frac{1}{s_{v} !} \gamma^{\delta\left(\left|P_{v}\right|\right)} \\
& J_{h, n}^{(5)}(\tau, \mathbf{P}, T) \leq(c|U|)^{n} \gamma^{h\left(\frac{7}{2}-\frac{3}{4}\left|P_{v_{0}}\right|\right)} \prod_{v \text { not e.p. }} \frac{1}{s_{v} !} \gamma^{\delta\left(\left|P_{v}\right|\right)} .
\end{aligned}
$$

The proof of Lemma 2.7 consists in a repetition of the proof of Theorem 2.1, unless for the fact that one has to use the estimates described in Lemma 2.6 in order to bound the sum over the sector indices. Lemma 2.6 guarantees exactly that the analogue of the sum in (2.103) with 3 external fixed sectors is bounded by the r.h.s. of (2.103) times a gain $\gamma^{h / 2}$ and the analogue of the sum in (2.103) with 5 external fixed sectors is bounded by the r.h.s. of (2.103) times $\gamma^{h}$, and (2.118) follows.

\subsection{The two point Schwinger function}

In this section we want to describe how to modify the expansion for the free energy described in previous sections in order to compute the two point Schwinger function.

The Schwinger functions (2.10) can be derived from the generating function defined as

$$
\mathcal{W}(\phi)=\log \int P(d \psi) e^{-\mathcal{V}(\psi)+\int d \mathbf{x}\left[\phi_{\mathbf{x}, \sigma}^{+} \psi_{\mathbf{x}, \sigma}^{-}+\psi_{\mathbf{x}, \sigma}^{+} \phi_{\mathbf{x}, \sigma}^{-}\right]}
$$

where the variables $\phi_{\mathbf{x}, \sigma}^{\varepsilon}$ are Grassmann variables, anticommuting among themselves and with the variables $\psi_{\mathbf{x}, \sigma}^{\varepsilon}$. In particular the two-point Schwinger function $S(\mathbf{x}-\mathbf{y}) \equiv S(\mathbf{x}, \sigma,-; \mathbf{y}, \sigma,+)$, see $(2.10)$, is given by

$$
S(\mathbf{x}-\mathbf{y})=\left.\frac{\partial^{2}}{\phi_{\mathbf{x}, \sigma}^{+} \phi_{\mathbf{y}, \sigma}^{-}} \mathcal{W}(\phi)\right|_{\phi=0} .
$$

We start by studying the generating function (2.119) and, in analogy with the procedure described in Section 2.2, we begin by decomposing the field $\psi$ in an ultraviolet and an infrared component: $\psi=\psi^{(1)}+\psi^{(\leq 0)}$, see (2.11). Proceeding through the analogues of (2.13) and (2.14), after the integration of the $\psi^{(1)}$ variables, we can rewrite:

$$
\begin{aligned}
e^{\mathcal{W}(\phi)}=e^{-L^{2} \beta F_{0}+S^{(\geq 0)}(\phi)} \int P\left(d \psi^{(\leq 0)}\right) & \\
\cdot & e^{-\mathcal{V}^{(0)}\left(\psi^{(\leq 0)}\right)-B^{(0)}\left(\psi^{(\leq 0)}, \phi\right)+\int d \mathbf{x}\left[\phi_{\mathbf{x}, \sigma}^{+} \psi_{\mathbf{x}, \sigma}^{(\leq 0)-}+\psi_{\mathbf{x}, \sigma}^{(\leq 0)+} \phi_{\mathbf{x}, \sigma}^{-}\right]},
\end{aligned}
$$

where $S^{(\geq 0)}(\phi)$ collects the terms depending on $\phi$ but not on $\psi^{(\leq 0)}$ and $B^{(0)}\left(\psi^{(\leq 0)}, \phi\right)$ the terms depending both on $\phi$ and $\psi^{(\leq 0)}$, at least quadratic in $\phi$.

Proceeding as in Section 2.3, we can show inductively (see below) that for any $h_{\beta} \leq h<0, e^{\mathcal{W}(\phi)}$ can be rewritten in a way similar to the r.h.s. of $(2.121)$ 
(and analogous to (2.18)):

$$
\begin{aligned}
& e^{\mathcal{W}(\phi)}=e^{-L^{2} \beta F_{h}+S^{(\geq h)}(\phi)} \int P_{E_{h}, C_{h}}\left(d \psi^{(\leq h)}\right) \\
& \cdot e^{-\mathcal{V}^{(h)}\left(\psi^{(\leq h)}\right)-B^{(h)}\left(\psi^{(\leq h)}, \phi\right)+\int d \mathbf{k}\left[\hat{\phi}_{\mathbf{k}, \sigma}^{+} \hat{Q}_{\mathbf{k}}^{(h+1)} \hat{\psi}_{\mathbf{k}, \sigma}^{(\leq h)-}+\hat{\psi}_{\mathbf{k}, \sigma}^{(\leq h)+} \hat{Q}_{\mathbf{k}}^{(h+1)} \hat{\phi}_{\mathbf{k}, \sigma}^{-}\right],}
\end{aligned}
$$

where $\int d \mathbf{k}$ must be interpreted as equal to $\frac{1}{\beta L^{2}} \sum_{\mathbf{k} \in \mathcal{D}_{\beta, L}}$ and $B^{(h)}\left(\psi^{(\leq h)}, \phi\right)$ can be written as $B_{\phi}^{(h)}\left(\psi^{(\leq h)}\right)+\bar{W}_{R}^{(h)}$, with $\bar{W}_{R}^{(h)}$ containing the terms of third or higher order in $\phi$ and $B_{\phi}^{(h)}\left(\psi^{(\leq h)}\right)$ of the form

$$
\begin{aligned}
\int d \mathbf{x}\left[\phi_{\cdot, \sigma}^{+}\right. & \left.* G^{(h+1)} * \frac{\partial \mathcal{V}^{(h)}\left(\psi^{(\leq h)}\right)}{\partial \psi(\leq h)+}+\frac{\partial \mathcal{V}^{(h)}\left(\psi^{(\leq h)}\right)}{\partial \psi_{\cdot, \sigma}^{(\leq h)-}} * G^{(h+1)} * \phi_{\cdot, \sigma}^{-}\right] \\
& +\int d \mathbf{x}\left[\phi_{\cdot, \sigma}^{+} * G^{(h+1)} * \frac{\partial^{2} \hat{\mathcal{V}}^{(h)}\left(\psi^{(\leq h)}\right)}{\partial \psi_{\cdot, \sigma}^{(\leq h)+} \partial \psi_{\cdot, \sigma^{\prime}}^{(\leq h)-}} * G^{(h+1)} * \phi_{\cdot, \sigma^{\prime}}^{-}\right]
\end{aligned}
$$

where

$$
G^{(h+1)}(\mathbf{x})=\sum_{j \geq h+1} g^{(k)} * Q^{(k)}(\mathbf{x})
$$

and $\hat{Q}_{\mathbf{k}}^{(h)}$ is defined inductively by the relations

$$
\hat{Q}_{\mathbf{k}}^{(h)}=\hat{Q}_{\mathbf{k}}^{(h+1)}-\hat{n}_{h}(\mathbf{k}) \hat{G}^{(h+1)}(\mathbf{k}), \quad Q_{\mathbf{k}}^{(1)} \equiv 1,
$$

with $\hat{n}_{h}(\mathbf{k})$ defined as in (2.23)-(2.24). Note that, by the compact support properties of $\hat{g}^{(h)}(\mathbf{k})$ and the bound in the first line of (2.38), it holds that (for $U$ small enough), if $\hat{g}^{(h)}(\mathbf{k}) \neq 0$, then $\hat{g}^{(j)}(\mathbf{k})=0$ for $|j-h|>2$, so that

$$
\hat{Q}_{\mathbf{k}}^{(h)}=1-\hat{n}_{h}(\mathbf{k}) \hat{g}^{(h+1)}(\mathbf{k}) \hat{Q}_{\mathbf{k}}^{(h+1)}-\hat{n}_{h+1}(\mathbf{k}) \hat{g}^{(h+2)}(\mathbf{k}) \hat{Q}_{\mathbf{k}}^{(h+2)} .
$$

Hence, proceeding by induction, we see that on the support of $\hat{g}^{(h)}(\mathbf{k})$ we have

$$
\left|\hat{Q}_{\mathbf{k}}^{(h)}-1\right| \leq C|U||h| \gamma^{h}, \quad\left|\partial_{\mathbf{k}}^{n} \hat{Q}_{\mathbf{k}}^{(h)}\right| \leq C_{n}|U||h| \gamma^{(1-n) h},
$$

uniformly in $h$. In order to derive (2.127), we used the inductive assumption (2.38).

Using (2.127) and the definition (2.124), it is easy to see that $G^{(h)}(\mathbf{x})$ satisfies bounds similar to (2.60) and (2.61); in particular, we find that

$$
\int d \mathbf{x}|\mathbf{x}|^{j}\left|G^{(h)}(\mathbf{x})\right| \leq C_{j} \gamma^{-(1+j) h} .
$$

For $h=0$ the assumption (2.122) is clearly true (it coincides with (2.121)). Assuming inductively that (2.122) is true up to a certain value of $h \leq 0$, we can show that the same representation is valid for $h-1$. In fact we can rewrite the term $\mathcal{V}^{(h)}$ in the exponent of (2.122) as $\mathcal{V}^{(h)}=\mathcal{L} \mathcal{V}^{(h)}+\mathcal{R} \mathcal{V}^{(h)}$, using (2.21), and we "absorb" the quadratic part of $\mathcal{L} \mathcal{V}^{(h)}$ (the one in the first line of (2.23)) in the fermionic integration, as explained in Section 2.3. Similarly we rewrite

$$
\frac{\partial}{\partial \psi_{\mathbf{x}, \sigma}^{(\leq h) \pm}} \mathcal{V}^{(h)}\left(\psi^{(\leq h)}\right)=\int d \mathbf{y} n_{h}(\mathbf{x}-\mathbf{y}) \psi_{\mathbf{y}, \sigma}^{(\leq h) \mp}+\frac{\partial}{\partial \psi_{\mathbf{x}, \sigma}^{(\leq h) \pm}} \hat{\mathcal{V}}^{(h)}\left(\psi^{(\leq h)}\right),
$$


where $\hat{\mathcal{V}}^{(h)}$ was defined after (2.26). This rewriting induces a decomposition of the first line of (2.123) into two pieces, the first proportional to $n_{h}$, the second identical to the first line of (2.123) itself, with $\mathcal{V}^{(h)}$ replaced by $\hat{\mathcal{V}}^{(h)}$. We choose to "absorb" the term proportional to $n_{h}$ into the definition of $Q^{(h)}$, and this gives the recursion relation $(2.125)$.

After these splittings and redefinitions, we integrate the field $\psi^{(h)}$, as in Section 2.3, and we end up with an expression given by the r.h.s. of (2.122), with $h$ replaced by $h-1$ and the inductive assumption (2.122) is proved.

For $h=h_{\beta}$ we define

$$
\begin{gathered}
e^{-L^{2} \beta\left(\tilde{F}_{h_{\beta}}+t_{h_{\beta}}\right)+S^{\left(<h_{\beta}\right)}(\phi)}=\int P_{E_{h_{\beta}-1}, C_{h_{\beta}}}\left(d \psi^{\left(\leq h_{\beta}\right)}\right) \\
\cdot \exp \left\{-\hat{\mathcal{V}}^{\left(h_{\beta}\right)}\left(\psi^{\left(\leq h_{\beta}\right)}\right)-B^{\left(h_{\beta}\right)}\left(\psi^{\left(\leq h_{\beta}\right)}, \phi\right)\right. \\
\left.+\int d \mathbf{k}\left[\hat{\phi}_{\mathbf{k}, s}^{+} \hat{Q}_{\mathbf{k}}^{\left(h_{\beta}+1\right)} \hat{\psi}_{\mathbf{k}, \sigma}^{\left(\leq h_{\beta}\right)-}+\hat{\psi}_{\mathbf{k}, \sigma}^{\left(\leq h_{\beta}\right)+} \hat{Q}_{\mathbf{k}}^{\left(h_{\beta}+1\right)} \hat{\phi}_{\mathbf{k}, \sigma}^{-}\right]\right\},
\end{gathered}
$$

so that, up to terms of third or higher order in $\phi$,

$$
\begin{aligned}
& S^{\left(<h_{\beta}\right)}(\phi)=\sum_{\sigma=\uparrow \downarrow} \int d \mathbf{x} . \\
& \quad\left(\phi_{\sigma}^{+} * Q^{\left(h_{\beta}\right)} * g^{\left(h_{\beta}\right)} * Q^{\left(h_{\beta}\right)} * \phi_{\sigma}^{-}-\phi_{\sigma}^{+} * G^{\left(h_{\beta}\right)} * n_{h_{\beta}-1} * G^{\left(h_{\beta}\right)} * \phi_{\sigma}^{-}\right) .
\end{aligned}
$$

From the definitions and the construction above, we get

$$
\begin{aligned}
& S(\mathbf{x}-\mathbf{y})=\left.\frac{\partial^{2}}{\phi_{\mathbf{x}, \sigma}^{+} \phi_{\mathbf{y}, \sigma}^{-}}\left[S^{\left(<h_{\beta}\right)}(\phi)+S^{\left(\geq h_{\beta}\right)}(\phi)\right]\right|_{\phi=0}= \\
& \sum_{h=h_{\beta}}^{1}\left[\left(Q^{(h)} * g^{(h)} * Q^{(h)}\right)(\mathbf{x}-\mathbf{y})-\left(G^{(h)} * n_{h-1} * G^{(h)}\right)(\mathbf{x}-\mathbf{y})\right] .
\end{aligned}
$$

Taking the Fourier transform and defining $h_{\mathbf{k}}=\min \left\{h: \hat{g}^{(h)}(\mathbf{k}) \neq 0\right\}$, we get

$$
\hat{S}(\mathbf{k})=\sum_{j=h_{\mathbf{k}}}^{h_{\mathbf{k}}+2} g^{(j)}(\mathbf{k})\left(Q_{\mathbf{k}}^{(j)}\right)^{2}-\sum_{j=h_{\mathbf{k}}}^{h_{\mathbf{k}}+2} G^{(j)}(\mathbf{k})^{2} \hat{n}_{j-1}(\mathbf{k}),
$$

where we used the compact support properties of $f_{j}(\mathbf{k})$, see comment after $(2.125)$. If we define $D_{h}(\mathbf{k})=-i k_{0}+E_{h-1}(\mathbf{k})-\mu$, we can rewrite $(2.133)$ as

$$
\hat{S}(\mathbf{k})=\frac{1}{D_{h_{\mathbf{k}}}(\mathbf{k})}[1+W(\mathbf{k})],
$$

where we used the identity, easily following from the definition (2.29),

$$
\sum_{j=h_{\mathbf{k}}}^{h_{\mathbf{k}}+2} f_{j}(\mathbf{k})=1
$$


and we defined

$$
\begin{aligned}
W(\mathbf{k})=\sum_{j=h_{\mathbf{k}}}^{h_{\mathbf{k}}+2} f_{j}(\mathbf{k})\left[\frac{D_{h_{\mathbf{k}}}(\mathbf{k})}{D_{j}(\mathbf{k})}\left(Q_{\mathbf{k}}^{(j)}\right)^{2}-1\right] \\
\quad-\sum_{j=h_{\mathbf{k}}}^{h_{\mathbf{k}}+2} \frac{\hat{n}_{j-1}(\mathbf{k})}{D_{h_{\mathbf{k}}}(\mathbf{k})}\left[\sum_{j^{\prime}=j}^{h_{\mathbf{k}}+2} f_{j^{\prime}}(\mathbf{k}) Q_{\mathbf{k}}^{\left(j^{\prime}\right)} \frac{D_{h_{\mathbf{k}}}(\mathbf{k})}{D_{j^{\prime}}(\mathbf{k})}\right]^{2} .
\end{aligned}
$$

By using $(2.24),(2.38)$ and $(2.127)$, it follows that

$$
|W(\mathbf{k})| \leq C|U||h| \gamma^{h}, \quad\left|\partial_{\mathbf{k}}^{n} W(\mathbf{k})\right| \leq C_{n}|U||h| \gamma^{(1-n) h} .
$$

Substituting (2.134) into the definition (1.6) of $\Sigma(\mathbf{k})$ we find:

$$
\Sigma(\mathbf{k})=E_{h_{\mathbf{k}}-1}(\mathbf{k})-\varepsilon_{0}(\vec{k})+\left[\frac{1}{1+W(\mathbf{k})}-1\right] D_{h_{\mathbf{k}}}(\mathbf{k}),
$$

so that, using (2.38) and (2.137), we find

$$
|\Sigma(\mathbf{k})| \leq C|U|, \quad\left|\partial_{\mathbf{k}}^{2} \Sigma(\mathbf{k})\right| \leq C c_{0} .
$$

In the same way we can prove that $\left|\partial_{\mathbf{k}} \Sigma(\mathbf{k})\right| \leq C|U|$, but this bound is not sufficient to prove Theorem 1.1, which needs the improved bound

$$
\left|\partial_{\mathbf{k}} \Sigma(\mathbf{k})\right| \leq C|U|^{2} .
$$

In order to get this estimate, one has to check that at first order $\Sigma(\mathbf{k})$ does not depend on k. By using (2.126), (2.135) and some straightforward calculation, one can see that

$$
\Sigma(\mathbf{k})=\sum_{j=h_{\mathbf{k}}-1}^{0} \hat{n}_{j}(\mathbf{k})+O\left(U^{2}\right) .
$$

Then (2.140) follows from the remark that the first order contribution to $n_{j}(\mathbf{k})$ (the tadpole graph) is constant.

Theorem 1.1 follows from the bounds (2.139) and (2.140), the remark that, by the same symmetries implying (2.40), $\sum_{j= \pm} \operatorname{Im} \Sigma\left(j \pi \beta^{-1}, \vec{k}\right)=0$ and $\sum_{j= \pm} j \operatorname{Re} \Sigma\left(j \pi \beta^{-1}, \vec{k}\right)=0$ and an explicit calculation of the second order contributions to $\Sigma(\mathbf{k})$ and its first derivatives with respect to $k_{0}$ and $\vec{k}$, showing that they are not vanishing, as well as the first order contribution to $\Sigma(\mathbf{k})$ (shown in (2.141)).

The second claim in the Remark after Theorem 1.1 on the spatial decay of $S(\mathbf{x})$ follows from bounding (2.132) and its derivatives, in a way similar to that described in Sec. 5 of $[\mathrm{BGM}]$ or in Sec. 12 of $[\mathrm{GM}]$. We do not repeat here the details.

Of course, the results above are obtained under the assumption (2.38) and the assumption that (2.92) is true for any $h_{i}-1 \geq h_{\beta}$, that we shall inductively prove in the next section. 


\section{Smoothness of the effective dispersion relation}

\subsection{Proof of the bounds (2.38)}

In this section we first want to prove (2.38), under the assumption that the $\lambda$ smallness condition (2.92) is verified for any $h_{i}>h$; we shall then prove the smallness assumption on $\lambda_{h}$ by an iterative argument.

We actually want to prove a statement slightly stronger than (2.38) and, in order to do this, we introduce some definitions. We denote by $\beta_{h}^{2}(\mathbf{x})$ the Fourier transform of $\hat{\beta}_{h}^{2}(\mathbf{k})=\hat{n}_{h}(\mathbf{k})$, see (2.36) and (2.24), and, given an isotropic sector index $\bar{\omega} \in \bar{O}_{h}$, we define

$$
\beta_{h, \bar{\omega}}^{2}(\mathbf{x}) \stackrel{\text { def }}{=}\left(\overline{\mathfrak{F}}_{2, h,(\bar{\omega}, \bar{\omega})} * \beta_{h}^{2}\right)(\mathbf{x})
$$

where $\overline{\mathfrak{F}}_{2, h,(\bar{\omega}, \bar{\omega})}$ is the "isotropic analogue" of the operator in (2.90) (i.e., the operator obtained by replacing $\tilde{F}_{h, \omega_{i}}(\vec{k})$ in the r.h.s. of $(2.90)$ with $\tilde{\bar{F}}_{h, \bar{\omega}}(\vec{k})$, where $\tilde{\bar{F}}_{h, \bar{\omega}}(\vec{k})$ is a smooth function $=1$ on $\bar{S}_{h, \bar{\omega}}$ and with a support slightly larger than $\left.\bar{S}_{h, \bar{\omega}}\right)$. We want to prove the following.

Theorem 3.1. Given $h_{\beta} \leq h \leq 0$, let us assume that $E_{j}(\mathbf{k})$ satisfies (2.38) for any $j \geq h$ and that, given any tree $\tau \in \mathcal{T}_{h, n}$ (with all its labels), $\tilde{\lambda}_{h_{v^{*}-1, \Omega_{v^{*}}} \text { satisfies }}$ (2.92) for all the endpoints $v^{*} \in \tau$. Then, if $U_{0}\left|h_{\beta}\right|=c_{0}$ is small enough,

$$
\begin{aligned}
& \int d \mathbf{x}\left|\beta_{h, \bar{\omega}}^{2}(\mathbf{x})\right| \leq C_{0}|U||h| \gamma^{2 h}, \\
& \int d \mathbf{x}|\mathbf{x}|^{n}\left|\beta_{h, \bar{\omega}}^{2}(\mathbf{x})\right| \leq C_{n}|U|^{2}|h| \gamma^{(2-n) h}, \quad n \geq 1 .
\end{aligned}
$$

Note that the bound (2.38) easily follows from Theorem 3.1. In fact, given $\mathbf{k}$ in the support of $C_{h}^{-1}(\mathbf{k})$, let us define $\bar{\omega}(\vec{k}) \in \bar{O}_{h}$ as the isotropic sector index such that $\tilde{\bar{F}}_{h, \bar{\omega}(\vec{k})}(\vec{k})=1$, so that $\hat{\beta}_{h}^{2}(\mathbf{k})=\tilde{\bar{F}}_{h, \bar{\omega}(\vec{k})}(\vec{k}) \hat{\beta}_{h}^{2}(\mathbf{k})$. Hence, we can write

$$
\begin{aligned}
& \hat{\beta}_{h}^{2}(\mathbf{k})=\int d \mathbf{x} e^{i \mathbf{k} \mathbf{x}} \beta_{h, \bar{\omega}}^{2}(\mathbf{x}) \\
& \partial_{k_{i_{1}}} \cdots \partial_{k_{i_{n}}}\left(\hat{\beta}_{h}^{2}(\mathbf{k})\right)=i^{n} \int d \mathbf{x} x_{i_{1}} \cdots x_{i_{n}} e^{i \mathbf{k x}} \beta_{h, \bar{\omega}}^{2}(\mathbf{x}) .
\end{aligned}
$$

The bounds (2.38) immediately follow from these identities, together with the definition of $C_{h}^{-1}(\mathbf{k})$ and the bounds $(3.2)$.

So now we shall focus on the proof of Theorem 3.1 .

Proof. The same iterative construction leading to the tree expansion for $\mathcal{V}^{(h)}$ allows us to represent $\beta_{h, \bar{\omega}}^{2}(\mathbf{x})$ as a sum over trees. Let $\mathbf{x}, \mathbf{y}$ be the two points where the two external fields (the fields in $P_{v_{0}}$ ) are hooked on; then, by $(2.23),(2.24)$ and $(2.87)$, 


$$
\begin{aligned}
\beta_{h, \bar{\omega}}^{2}(\mathbf{x}-\mathbf{y})=\sum_{n=1}^{\infty} \sum_{\tau \in \mathcal{T}_{h-1, n}} \sum_{\substack{\mathbf{P} \in \mathcal{P}_{\tau} \\
\left|P_{v_{0}}\right|=2}} \sum_{T \in \mathbf{T}} \sum_{\Omega \backslash \Omega_{v_{0}}}\left[\prod_{v} \chi\left(\Omega_{v}\right)\right] \\
\cdot \overline{\mathfrak{F}}_{2, h,(\bar{\omega}, \bar{\omega})} * \int d\left(\mathbf{x}_{v_{0}} \backslash\{\mathbf{x}, \mathbf{y}\}\right) W_{\tau, \mathbf{P}, \Omega, T}^{(\mathrm{mod})}\left(\mathbf{x}_{v_{0}}\right),
\end{aligned}
$$

where $W_{\tau, \mathbf{P}, \Omega, T}^{(\bmod )}\left(\mathbf{x}_{v_{0}}\right)$ can be represented as in (2.88), with the kernels $K_{v_{i}^{*}}^{h_{i}}$ replaced by the modified ones. Again, for simplicity, we shall explicitly study only contributions coming from trees $\tau$ such that all the endpoints are of type $\lambda$. Given $\tau \in \mathcal{T}_{h-1, n}, \mathbf{P} \in \mathcal{P}_{\tau}$ with $\left|P_{v_{0}}\right|=2, T \in \mathbf{T}$, in analogy with definition (2.96), we define $\bar{J}_{h, n, \bar{\omega}}^{(2)}(\tau, \mathbf{P}, T ; j)$ as

$$
\begin{aligned}
\bar{J}_{h, n, \bar{\omega}}^{(2)}(\tau, \mathbf{P}, T ; j)=\sum_{\Omega \backslash \Omega_{v_{0}}} & {\left[\prod_{v} \chi\left(\Omega_{v}\right)\right] \int d(\mathbf{x}-\mathbf{y})|\mathbf{x}-\mathbf{y}|^{j} } \\
\cdot \mid & \left|\overline{\mathfrak{F}}_{2, h,(\bar{\omega}, \bar{\omega})} * \int d\left(\mathbf{x}_{v_{0}} \backslash\{\mathbf{x}, \mathbf{y}\}\right) W_{\tau, \mathbf{P}, \Omega, T}^{(\bmod )}\left(\mathbf{x}_{v_{0}}\right)\right| .
\end{aligned}
$$

We want to prove an improved version of the bound (2.97), valid if $\left|P_{v_{0}}\right|=2$ and $n \geq 2$ :

$$
\sup _{\bar{\omega} \in \bar{O}_{h}} \bar{J}_{h, n, \bar{\omega}}^{(2)}(\tau, \mathbf{P}, T ; j) \leq\left(c_{j}|U|\right)^{n}|h| \gamma^{(2-j) h} \prod_{v \in V_{c}} \frac{1}{s_{v} !}\left|P_{v}\right|^{5} \gamma^{\delta\left(\left|P_{v}\right|\right)\left(h_{v}-h_{v^{\prime}}\right)},
$$

where $v^{\prime}$ is the $c$-vertex immediately preceding $v$ on $\tau$. Note that the proof of Theorem 2.1, adapted to the present case, would easily imply a bound like (3.6) with $|h| \gamma^{2 h}$ replaced by $\gamma^{h}$ and $\left|P_{v}\right|^{5}$ replaced by 1 . The bound (3.6) is valid for $n \geq 2$; if $n=1$ there is only one possible choice of $\tau \in \mathcal{T}_{h, 1}$ and, calling $v$ the only endpoint in $\tau$ and $\left\{f_{2}, f_{3}\right\}=P_{v} \backslash P_{v_{0}}$, we shall prove that

$$
\begin{array}{r}
\bar{J}_{h, 1, \bar{\omega}}^{(2)}(\tau, \mathbf{P}, T ; j)=\sum_{\omega\left(f_{2}\right), \omega\left(f_{3}\right) \in O_{h}} \delta_{\omega\left(f_{2}\right), \omega\left(f_{3}\right)} \int d\left(\mathbf{x}_{1}-\mathbf{x}_{4}\right)\left|\mathbf{x}_{1}-\mathbf{x}_{4}\right|^{j} \\
\cdot\left|\overline{\mathfrak{F}}_{2, h,(\bar{\omega}, \bar{\omega})} * \int d \mathbf{x}_{2} d \mathbf{x}_{3} g_{\omega\left(f_{3}\right)}^{(h)}\left(\mathbf{x}_{2}-\mathbf{x}_{3}\right) \tilde{\lambda}_{h, \Omega_{v}}\left(\mathbf{x}_{1}, \mathbf{x}_{2}, \mathbf{x}_{3}, \mathbf{x}_{4}\right)\right| \\
\leq c_{j}|U|^{2-\delta_{j, 0}}|h|^{\delta_{j, 0}} \gamma^{(2-j) h} .
\end{array}
$$

It is easy to realize that the bounds (3.6) and (3.7) and the representation (3.4) allow us to get the bound (3.2) on $\beta_{h, \bar{\omega}}^{2}$. So we will now prove (3.6) and (3.7). We shall proceed as follows; we shall first prove (3.6) under the assumption that, given $h^{\prime}$ and $k$ with $h \leq h^{\prime} \leq k, \bar{\omega}_{1}, \bar{\omega}_{4} \in \bar{O}_{k}$, with $\left|\bar{\omega}_{1}-\bar{\omega}_{4}\right| \leq 1$, and $P=P^{i} \cup P^{a}$, with $P^{i}=\left\{f_{1}, f_{4}\right\}, P^{a}=\left\{f_{2}, f_{3}\right\}$, 


$$
\begin{aligned}
\sum_{\omega \in O_{h^{\prime}}} \int d\left(\mathbf{x}_{1}-\mathbf{x}_{4}\right)\left|\mathbf{x}_{1}-\mathbf{x}_{4}\right|^{j} \mid \int d \mathbf{x}_{2} d \mathbf{x}_{3} g_{\omega}^{\left(h^{\prime}\right)}\left(\mathbf{x}_{2}-\mathbf{x}_{3}\right) \\
\left.\cdot \tilde{\lambda}_{k, \tilde{\Omega}_{4}}\left(\mathbf{x}_{1}, \mathbf{x}_{2}, \mathbf{x}_{3}, \mathbf{x}_{4}\right)\left|\leq c_{j}\right| U\right|^{2-\delta_{j, 0}}\left(1+|k|^{\delta_{j, 0}}\right) \gamma^{2 h^{\prime}-j k}
\end{aligned}
$$

where $\tilde{\Omega}_{4}=\left\{\bar{\omega}_{f} \in \bar{O}_{k}, f \in P^{i}\right\} \cup\left\{\omega(f)=\omega, f \in P^{a}\right\}$ and, with a small abuse of notation,

$$
\begin{aligned}
& \tilde{\lambda}_{k, \tilde{\Omega}_{4}}\left(\mathbf{x}_{1}, \mathbf{x}_{2}, \mathbf{x}_{3}, \mathbf{x}_{4}\right)=\frac{1}{\beta^{4}} \sum_{\underline{k}_{0}} \int \frac{d \underline{k}}{(2 \pi)^{8}} e^{-i \sum_{i=1}^{4} \mathbf{k}_{i} \mathbf{x}_{i}} \\
& \cdot \tilde{\bar{F}}_{k, \bar{\omega}_{f_{1}}}\left(\vec{k}_{1}\right) \tilde{F}_{h^{\prime}, \omega}\left(\vec{k}_{2}\right) \tilde{F}_{h^{\prime}, \omega}\left(\vec{k}_{3}\right) \tilde{\bar{F}}_{k, \bar{\omega}_{f_{4}}}\left(\vec{k}_{4}\right) \hat{\lambda}_{k}\left(\mathbf{k}_{1}, \mathbf{k}_{2}, \mathbf{k}_{3}, \mathbf{k}_{4}\right) .
\end{aligned}
$$

After having proved (3.6) under the assumption (3.8), we shall prove (3.8) inductively in $h^{\prime}$, so that, in particular, (3.7) will follow.

In order to prove (3.6), we will first further expand $W_{\tau, \mathbf{P}, \Omega, T}^{(\bmod )}(\mathbf{x})$ in $(3.5)$ by extracting some "loop propagators" from the Gram determinant in the second line of (2.99) (via an interpolation technique described below). Such extraction of loop propagators will imply some new constraints on the sector indices appearing in the sum $\sum_{\Omega \backslash \Omega_{v_{0}}}$ in (3.5). Then, depending on the explicit structure of the terms obtained by this further expansion, we shall decide whether rewriting the extracted loop propagators as sums of isotropic propagators or not. In both cases we shall describe a new inductive procedure to bound the sum over the sector indices, in order to take into account the new constraints coming from the extraction of the loop propagators. We shall compare the new inductive procedure to bound the sector sums with the one described in Section 2.8 and by comparison we will show how to get the desired dimensional gains.

We can now turn to the proof of (3.6) and we shall first consider the case $j=0$. The proof is done by distinguishing among several different cases. First of all, we need to introduce some definitions. We call $\underline{v}_{0}=\left\{v_{1}, \ldots, v_{s_{v_{0}}}\right\}$ the set of $c$-vertices immediately following $v_{0}$ on $\tau$ (note that, for the trees contributing to $\beta_{h, \bar{\omega}}^{2}(\mathbf{x}-\mathbf{y}), v_{0}$ is necessarily a $c$-vertex). Given $T_{v_{0}}$, we identify the two (possibly coinciding) clusters $v_{\mathbf{x}}$ and $v_{\mathbf{y}}$ in which the two fields in $P_{v_{0}}$ (call them $f_{\mathbf{x}}, f_{\mathbf{y}}$ ) enter. Correspondingly, we call $l_{\mathbf{x}, \mathbf{y}}$ the (possibly trivial) path on $T_{v_{0}}$ connecting $v_{\mathbf{x}}$ and $v_{\mathbf{y}}$. Moreover we identify $v_{\mathbf{x}}$ with the root of $T_{v_{0}}$ : in this way the concept of leaf of $T_{v_{0}}$ is well defined.

(A) Let us assume that $s_{v_{0}} \geq 2$. Let us further distinguish the case $T_{v_{0}} \neq l_{\mathbf{x}, \mathbf{y}}$ and $T_{v_{0}} \equiv l_{\mathbf{x}, \mathbf{y}}$.

(A1) $s_{v_{0}} \geq 2$ and $T_{v_{0}} \neq l_{\mathbf{x}, \mathbf{y}}$. In this case there must be at least one leaf of $T_{v_{0}}$ different from $v_{\mathbf{y}}$; let us choose one such leaf and call it $v^{*}$; see Fig. 2 , where the solid lines represent the tree propagators in $T_{v_{0}}$, the wiggling lines represent the loop fields and the broken lines represent the external fields. Note that in Fig. 2 

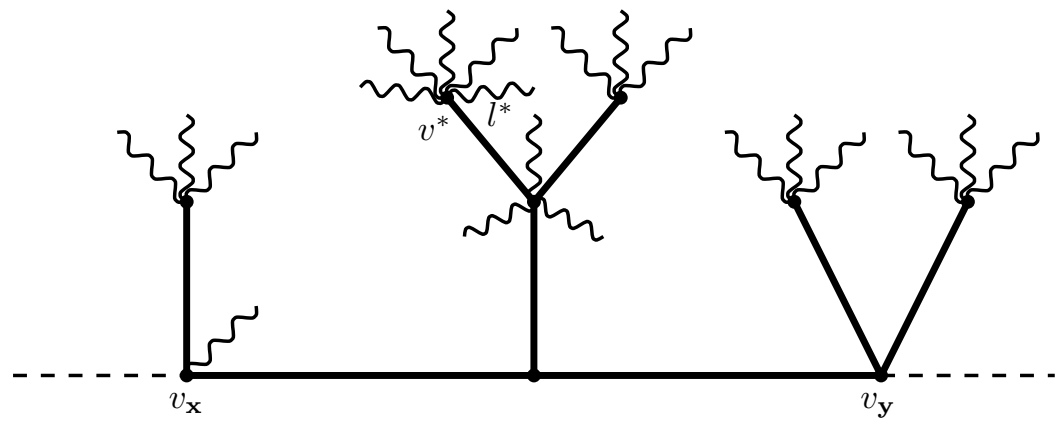

Figure 2. An example of case (A1).

the case $v_{\mathbf{x}} \neq v_{\mathbf{y}}$ is considered, but the following analysis is independent of this condition.

By construction, $v^{*}$ has one exiting line belonging to the spanning tree $T_{v_{0}}$ (call it $l^{*}$ and $f^{*}$ its field label) and $\left|P_{v^{*}}\right|-1 \geq 3$ loop lines. Let $\tilde{P}_{\underline{v}_{0}} \subset \cup_{v \in \underline{v}_{0}} P_{v}$ be the set of fields contracted into the Gram determinant $\operatorname{det} G^{h_{v_{0}}, \bar{T}_{v_{0}}}$ appearing in (2.88). If, given a set $\mathcal{I}$ of field labels, we define $\mathcal{I}^{ \pm}=\{f \in \mathcal{I}: \varepsilon(f)= \pm\}$, we can think $G^{h_{v_{0}}, T_{v_{0}}}$ as a matrix whose rows are associated to the fields $f \in \tilde{P}_{\underline{v}_{0}}^{-}$and whose columns are associated to the fields $f \in \tilde{P}_{\underline{v}_{0}}^{+}$. Let us distinguish the field labels belonging to $\tilde{P}_{v^{*}} \equiv P_{v^{*}} \backslash f^{*}$ from those belonging to $\tilde{P}_{\underline{v}_{0}} \backslash \tilde{P}_{v^{*}}$ and let us correspondingly write $G^{h, T_{v_{0}}}$ in blocks, as follows:

$$
\operatorname{det} G^{h, T_{v_{0}}}=\operatorname{det}\left(\begin{array}{cc}
A & B \\
C & D
\end{array}\right)
$$

where: $A$ is the block with both row and column indices belonging to $\tilde{P}_{v^{*}} ; B$ is the block with row indices in $\tilde{P}_{v^{*}}$ and column indices in $\tilde{P}_{\underline{v}_{0}} \backslash \tilde{P}_{v^{*}} ; C$ is the block with row indices in $\tilde{P}_{\underline{v}_{0}} \backslash \tilde{P}_{v^{*}}$ and column indices in $\tilde{P}_{v^{*}} ; D$ is the block with both row and column indices belonging to $\tilde{P}_{\underline{v}_{0}} \backslash \tilde{P}_{v^{*}}$. Note that by construction neither $A$ nor $D$ are square matrices and, because of this, $\operatorname{det}\left(\begin{array}{cc}A & 0 \\ 0 & D\end{array}\right)=0$, so that we can rewrite the r.h.s. of (3.10) as:

$$
\begin{aligned}
\operatorname{det}\left(\begin{array}{cc}
A & B \\
C & D
\end{array}\right) & -\operatorname{det}\left(\begin{array}{cc}
A & 0 \\
0 & D
\end{array}\right)=\int_{0}^{1} d s \frac{\mathrm{d}}{\mathrm{d} s} \operatorname{det}\left(\begin{array}{cc}
A & s B \\
s C & D
\end{array}\right) \\
= & \int_{0}^{1} d s \sum_{i \in \tilde{P}_{v^{*}}^{-},} \sum_{j \in \tilde{P}_{\underline{v}_{0}}^{+} \backslash \tilde{P}_{v^{*}}^{+}}(-1)^{i+j} B_{i j} \operatorname{det} m_{i j}(G(s)) \\
& +\int_{0}^{1} d s \sum_{i \in \tilde{P}_{\underline{v}_{0}}^{-} \backslash \tilde{P}_{v^{*}}^{-}, j \in \tilde{P}_{v^{*}}^{+}}(-1)^{i+j} C_{i j} \operatorname{det} m_{i j}(G(s)),
\end{aligned}
$$


where $G(s)=\left(\begin{array}{cc}A & s B \\ s C & D\end{array}\right)$ and, given a matrix $M, m_{i j}(M)$ is the minor of $M$ corresponding to the entry $i, j$. The identity (3.11) is essentially a first order expansion of $\operatorname{det}\left(\begin{array}{ll}A & B \\ C & D\end{array}\right)$ around the point $\left(\begin{array}{cc}A & 0 \\ 0 & D\end{array}\right)$ and it corresponds to the operation of "extracting one loop" connecting $\tilde{P}_{v^{*}}$ with $\tilde{P}_{\underline{v}_{0}} \backslash \tilde{P}_{v^{*}}$. The key remark is that the r.h.s. of (3.11) is a sum of less than $\prod_{v \in v_{0}}\left|\underline{P}_{v}\right|$ terms, each of them equal to the product of a propagator $B_{i j}$ or $C_{i j}$ times a determinant that is still a Gram determinant, then it can still be bounded via the Gram-Hadamard inequality, the result being the following dimensional estimate, analogue to the r.h.s. of (2.100):

$$
\left|\operatorname{det} m_{j i}(G(s))\right| \leq c^{\sum_{i=1}^{s_{v_{0}}}\left|P_{v_{i}}\right|-\left|P_{v}\right|-2 s_{v_{0}}} \gamma^{\frac{3}{4} h\left(\sum_{i=1}^{s_{v_{0}}}\left|P_{v_{i}}\right|-\left|P_{v}\right|-2 s_{v_{0}}\right)} .
$$

A procedure analogue to the first order expansion leading to (3.11) allows us to expand $\left(\begin{array}{ll}A & B \\ C & D\end{array}\right)$ up to fifth order around the point $\left(\begin{array}{cc}A & 0 \\ 0 & D\end{array}\right)$ and to rewrite $\operatorname{det} G^{h, T_{v_{0}}}$ as

$$
\begin{aligned}
& \operatorname{det} G^{h, T_{v_{0}}}\left(\mathbf{t}_{v_{0}}\right)=\sum_{\left\{i_{1} j_{1}, i_{1}^{\prime} j_{1}^{\prime}\right\}}^{*}(-1)^{\varepsilon_{1}} G_{i_{1} j_{1}, i_{1}^{\prime} j_{1}^{\prime}}^{h, T_{v_{0}}}\left(\mathbf{t}_{v_{0}}\right) \\
& \cdot \operatorname{det} \widetilde{G}^{h, T_{v_{0}}}\left(\left\{i_{1} j_{1}, i_{1}^{\prime} j_{1}^{\prime}\right\} ; 0, \mathbf{t}_{v_{0}}\right)+\frac{1}{3 !} \sum_{\left\{i_{q} j_{q}, i_{q}^{\prime} j_{q}^{\prime}\right\}_{q=1}^{3}}^{*}(-1)^{\varepsilon_{1}+\varepsilon_{2}+\varepsilon_{3}} \\
& \cdot\left[\prod_{q=1}^{3} G_{i_{q} j_{q}, i_{q}^{\prime} j_{q}^{\prime}}^{h, T_{v_{0}}}\left(\mathbf{t}_{v_{0}}\right)\right] \operatorname{det} \widetilde{G}^{h, T_{v_{0}}}\left(\left\{i_{q} j_{q}, i_{q}^{\prime} j_{q}^{\prime}\right\}_{q=1}^{3} ; 0, \mathbf{t}_{v_{0}}\right) \\
& +\frac{1}{5 !} \sum_{\left\{i_{q} j_{q}, i_{q}^{\prime} j_{q}^{\prime}\right\}_{q=1}^{5}}^{*}(-1)^{\varepsilon_{1}+\cdots+\varepsilon_{5}}\left[\prod_{q=1}^{5} G_{i_{q} j_{q}, i_{q}^{\prime} j_{q}^{\prime}}^{h, T_{v_{0}}}\left(\mathbf{t}_{v_{0}}\right)\right] \\
& \cdot \operatorname{det} \widetilde{G}^{h, T_{v_{0}}}\left(\left\{i_{q} j_{q}, i_{q}^{\prime} j_{q}^{\prime}\right\}_{q=1}^{5} ; \bar{s}, \mathbf{t}_{v_{0}}\right)
\end{aligned}
$$

where: the $*$ 's on the three sums constraint the indices $\left\{i_{q} j_{q}, i_{q}^{\prime} j_{q}^{\prime}\right\}$ to run over choices such that $f_{i_{q} j_{q}}^{-} \in \tilde{P}_{v^{*}}$ and $f_{i_{q}^{\prime} j_{q}^{\prime}}^{+} \in \tilde{P}_{\underline{v}_{0}} \backslash \tilde{P}_{v^{*}}$ or vice versa; $\varepsilon_{q}= \pm$ is a sign, depending on the parity of the element index $\left\{i_{q} j_{q}, i_{q}^{\prime} j_{q}^{\prime}\right\}, q=1, \ldots, 5$; the matrices $\widetilde{G}^{h, T_{v_{0}}}\left(\left\{i_{q} j_{q}, i_{q}^{\prime} j_{q}^{\prime}\right\}_{q=1}^{L} ; s, \mathbf{t}_{v_{0}}\right), L=1,3,5$ are the minors of $G^{h, T_{v_{0}}}\left(s, \mathbf{t}_{v_{0}}\right)$ complementary to the elements $\left(i_{q} j_{q}, i_{q}^{\prime} j_{q}^{\prime}\right), q=1, \ldots, L$; the parameter $0 \leq \bar{s} \leq 1$ appearing in the argument of $\widetilde{G}^{h, T_{v_{0}}}$ in the third line is an interpolation parameter corresponding to the integration variable $s$ appearing in (3.11). Note that the determinants on the first two lines are computed at $s=0$, and this implies that they are the product of two determinants, the first involving only fields in $\tilde{P}_{v^{*}}$, the second involving only fields in $\tilde{P}_{\underline{v}_{0}} \backslash \tilde{P}_{v^{*}}$. The three determinants in (3.13) are Gram determinants which can be bounded using Gram-Hadamard inequality: the 
one on the first line can be bounded exactly as in (3.12), while the other two can be bounded by the r.h.s. of (3.12) times a factor $\gamma^{-3 h}$ or $\gamma^{-6 h}$ respectively. $=2$ :

The splitting (3.13) induces a similar splitting in $\bar{J}_{h, n, \bar{\omega}}^{(2)}(\tau, \mathbf{P}, T ; 0)$ with $\left|P_{v_{0}}\right|$

$$
\bar{J}_{h, n, \bar{\omega}}^{(2)}(\tau, \mathbf{P}, T ; 0) \leq \sum_{L=1,3,5} \bar{J}_{h, n, \bar{\omega}}^{(2)}(\tau, \mathbf{P}, T ; 0, L)
$$

and, calling $s_{L}=\delta_{L, 5} \bar{s}$

$$
\begin{aligned}
& \bar{J}_{h, n, \bar{\omega}}^{(2)}(\tau, \mathbf{P}, T ; 0, L)=\sum_{\Omega \backslash \Omega_{v_{0}}}\left[\prod_{v} \chi\left(\tilde{\Omega}_{v, \bar{\omega}}\right)\right] \mid \int \prod_{l \in T^{*}} d\left(\mathbf{x}_{l}-\mathbf{y}_{l}\right) \\
& \cdot\left[\prod_{v \text { e.p. }} \tilde{\lambda}_{h_{v}-1, \tilde{\Omega}_{v, \bar{\omega}}}\left(\mathbf{x}_{v}\right)\right]\left\{\int d P_{T_{v_{0}}}\left(\mathbf{t}_{v_{0}}\right) \frac{1}{s_{v_{0}} !} \frac{1}{L !}\right. \\
& \sum_{\left\{i_{q} j_{q}, i_{q}^{\prime} j_{q}^{\prime}\right\}_{q=1}^{L}}^{*}\left[\prod_{q=1}^{L} G_{i_{q} j_{q}, i_{q}^{\prime} j_{q}^{\prime}}^{h, T_{v_{0}}}\left(\mathbf{t}_{v_{0}}\right)\right] \\
& \left.\operatorname{det} \widetilde{G}^{h, T_{v_{0}}}\left(\left\{i_{q} j_{q}, i_{q}^{\prime} j_{q}^{\prime}\right\}_{q=1}^{L} ; s_{L}, \mathbf{t}_{v_{0}}\right) \prod_{l \in T_{v_{0}}} g_{\omega_{l}}^{(h)}\left(\mathbf{x}_{l}-\mathbf{y}_{l}\right) \delta_{\omega_{l}^{+}, \omega_{l}^{-}}\right\} \\
& \cdot\left\{\int d P_{T_{v}}\left(\mathbf{t}_{v}\right) \prod_{\substack{v>v_{0} \\
\text { not e. p. }}} \frac{1}{s_{v} !} \operatorname{det} G^{h_{v}, T_{v}}\left(\mathbf{t}_{v}\right) \prod_{l \in T_{v}} g_{\omega_{l}}^{\left(h_{v}\right)}\left(\mathbf{x}_{l}-\mathbf{y}_{l}\right) \delta_{\omega_{l}^{+}, \omega_{l}^{-}}\right\} \mid,
\end{aligned}
$$

where

$$
\tilde{\Omega}_{v, \bar{\omega}}=\left\{\omega(f) \in O_{h(f)}, f \in P_{v} \backslash\left\{f_{\mathbf{x}}, f_{\mathbf{y}}\right\}\right\} \cup\left\{\bar{\omega}_{f} \equiv \bar{\omega}, f \in P_{v} \cap\left\{f_{\mathbf{x}}, f_{\mathbf{y}}\right\}\right\}
$$

and, by writing $\chi\left(\tilde{\Omega}_{v, \bar{\omega}}\right)$, we implicitly introduced the notion of a $\chi$ function depending on a set of sector indices which contains both isotropic and anisotropic sector indices: in general, if $P_{v}^{a} \subset P_{v}, P_{v}^{i}=P_{v} \backslash P_{v}^{a}$ and $\Omega_{v}^{\prime}$ is the set of sector indices $\Omega_{v}^{\prime}=\left\{\omega(f) \in O_{h(f)}, f \in P_{v}^{a}\right\} \cup\left\{\bar{\omega}_{f} \in \bar{O}_{j_{f}}, f \in P_{v}^{i}\right\}$ labelled by $P_{v}^{a}$ and $P_{v}^{i}$, we define

$$
\chi\left(\Omega_{v}^{\prime}\right)=\chi\left(\forall f \in P_{v}, \exists \vec{k}(f) \in\left\{\begin{array}{ll}
S_{h(f), \omega(f)}, & \text { if } f \in P_{v}^{a} \\
\bar{S}_{j_{f}, \bar{\omega}_{f}}, & \text { if } f \in P_{v}^{i}
\end{array} \quad: \sum_{f \in P_{v}} \varepsilon(f) \vec{k}(f)=0\right) .\right.
$$

The modified coupling function $\tilde{\lambda}_{h_{v}-1, \tilde{\Omega}_{v, \bar{\omega}}}\left(\mathbf{x}_{v}\right)$ is defined in a way similar to (3.9). The presence of the functions $\chi\left(\tilde{\Omega}_{v, \bar{\omega}}\right)$ and of $\tilde{\lambda}_{h_{v}-1, \tilde{\Omega}_{v, \bar{\omega}}}\left(\mathbf{x}_{v}\right)$ is due to the convolution operator $\overline{\mathfrak{F}}_{2, h,(\bar{\omega}, \bar{\omega})}$ appearing in the definition $(3.5)$.

We now want to show how to get the desired dimensional gain for the three contributions $\bar{J}_{h, n, \bar{\omega}}^{(2)}(\tau, \mathbf{P}, T ; 0, L)$ in $(3.15), L=1,3,5$. In order to do that, we 
shall suppose that the modified coupling functions satisfy the following bound, essentially equivalent to the smallness condition (2.92):

$$
\frac{1}{L^{2} \beta} \int d \mathbf{x}_{v}\left|\tilde{\lambda}_{h_{v}-1, \tilde{\Omega}_{v, \bar{\omega}}}\left(\mathbf{x}_{v}\right)\right| \leq C|U| .
$$

At the end, by an iterative argument, we shall prove this bound, together with the bound (3.8).

(A1.1) $L=3$ : there are exactly three loop lines connecting $\tilde{P}_{v^{*}}$ with $\tilde{P}_{v_{0}} \backslash \tilde{P}_{v^{*}}$, call them $l_{1}, l_{2}, l_{3}$ and $f_{1}, f_{2}, f_{3}$ their field labels, see Fig. 3 .

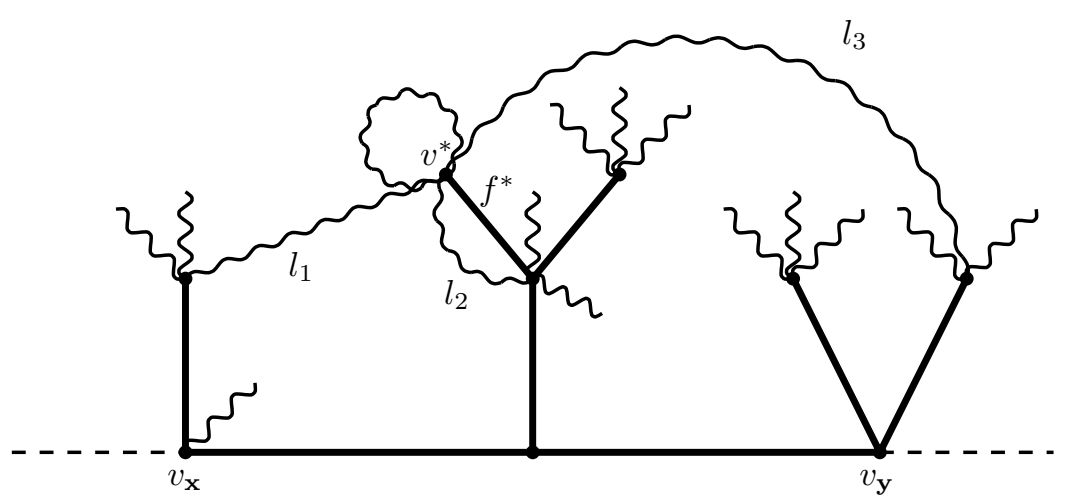

Figure 3. An example of case (A1.1).

Let us consider the product $\prod_{q=1}^{3} G_{i_{q} j_{q}, i_{q}^{\prime} j_{q}^{\prime}}^{h, T_{v_{0}}}=\prod_{q=1}^{3} t_{i_{q}, i_{q}^{\prime}} g_{\omega\left(f_{q}\right)}^{(h)}\left(\mathbf{x}_{l_{q}}-\mathbf{y}_{l_{q}}\right)$ $\delta_{\omega_{l_{q}}^{+}, \omega_{l_{q}}^{-}}$in (3.15) and let us substitute both the extracted loop propagators $g_{\omega\left(f_{q}\right)}^{(h)}$ with $q=1,2,3$ and the propagator of the spanning tree $g_{\omega\left(f^{*}\right)}^{(h)}$ (we recall that $f^{*}$ is the field label of the line $l^{*}$ of $T_{v_{0}}$ exiting from $v^{*}$ ) with the sums of isotropic propagators $\bar{g}_{\bar{\omega}_{q}}^{(h)}, q=1,2,3$, and $\bar{g}_{\bar{\omega}^{*}}^{(h)}$, such that $\bar{S}_{h, \bar{\omega}_{q}} \subset S_{h, \omega\left(f_{q}\right)}$ and $\bar{S}_{h, \bar{\omega}^{*}} \subset S_{h, \omega\left(f^{*}\right)}$. This operation is allowed since $\sum_{\omega \in O_{h}} g_{\omega}^{(h)}=\sum_{\bar{\omega} \in \bar{O}_{h}} \bar{g}_{\bar{\omega}}^{(h)}$ and our definitions are such that any isotropic sector is strictly contained in one anisotropic sector.

For any fixed configuration of internal anisotropic sectors, the new sums on the isotropic sectors can be written as:

$$
\sum_{\substack{\bar{\omega}_{q} \prec \omega\left(f_{q}\right) \\ q=1,2,3}} \sum_{\bar{\omega}^{*} \prec \omega\left(f^{*}\right)},
$$

where the meaning of the symbol $\prec$ is the same as in (2.108). Note also that, by construction, the summand in the r.h.s. of (3.15) is 0 unless $\chi\left(\left\{\bar{\omega}_{1}, \bar{\omega}_{2}, \bar{\omega}_{3}, \bar{\omega}^{*}\right\}\right)=1$, so that we can freely multiply the summand in the r.h.s. of (3.15) by $\chi\left(\left\{\bar{\omega}_{1}, \bar{\omega}_{2}, \bar{\omega}_{3}, \bar{\omega}^{*}\right\}\right)$. 
Having done this, we can bound the resulting expression by replacing all the modified coupling functions, all the propagators and all the Gram determinants with their absolute values. Now, we can bound the integral over the absolute values of the modified coupling functions as in (2.102) (by using (3.18) in place of (2.92)), the integral over the absolute values of the propagators of the spanning tree as in (2.101) (note that the integral $\int d\left(\mathbf{x}_{l^{*}}-\mathbf{y}_{l^{*}}\right)\left|\bar{g}_{\bar{\omega}^{*}}^{(h)}\left(\mathbf{x}_{l^{*}}-\mathbf{y}_{l^{*}}\right)\right|$ admits the same dimensional bound as the integral of an anisotropic propagator, see Lemma 2.4). Moreover, the product of the determinants times $\prod_{q=1}^{3}\left|\bar{g}_{\bar{\omega}_{q}}^{(h)}\left(\mathbf{x}_{l_{q}}-\mathbf{y}_{l_{q}}\right)\right|$ can be bounded by the product over $v$ of the r.h.s. of (2.100) times a dimensional gain $\gamma^{3 h / 2}$ coming from the fact that the bound on the size of $\left|\bar{g}_{\bar{\omega}_{q}}^{(h)}\left(\mathbf{x}_{l_{q}}-\mathbf{y}_{l_{q}}\right)\right|$ is $\gamma^{h / 2}$ smaller than the bound on the size of an anisotropic propagator of scale $h$, see Lemma 2.2 and Lemma 2.4. After these bounds, the sum $\sum_{\left\{i_{q} j_{q}, i_{q}^{\prime} j_{q}^{\prime}\right\}_{q=1}^{3}}^{*}$ can be bounded by $\prod_{v \in \underline{v}_{0}}\left|P_{v}\right|^{3} \leq \prod_{v \in V_{c}}\left|P_{v}\right|^{5}$, where the latter is the same product appearing in the r.h.s. of (3.6).

We are still left with the sum over the sector indices:

$$
\sum_{\cup_{v} \Omega_{v} \backslash \Omega_{v_{0}}} \sum_{\substack{\bar{\omega}_{q} \prec \omega\left(f_{q}\right) \\ \bar{\omega}^{*} \prec \omega\left(f^{*}\right)}}\left[\prod_{\substack{v>v_{0} \\ v \in V_{c}}} \chi\left(\tilde{\Omega}_{v, \bar{\omega}}\right)\right] \chi\left(\left\{\bar{\omega}_{1}, \bar{\omega}_{2}, \bar{\omega}_{3}, \bar{\omega}^{*}\right\}\right)\left[\prod_{l \in T} \delta_{\omega_{l}^{+}, \omega_{l}^{-}}\right] \prod_{q=1}^{3} \delta_{\omega_{l_{q}}^{+}, \omega_{l_{q}}^{-}} .
$$

where the product $\chi\left(\left\{\bar{\omega}_{1}, \bar{\omega}_{2}, \bar{\omega}_{3}, \bar{\omega}^{*}\right\}\right) \prod_{q=1}^{3} \delta_{\omega_{l_{q}}^{+}, \omega_{l_{q}}^{-}}$takes into account the new constraints coming from the extraction of the three loop propagators.

We want to prove that (3.20) can be bounded by the r.h.s. of (2.103) times a dimensional factor $\gamma^{-h / 2}|h|$. Combining this loss with the gain $\gamma^{3 h / 2}$ coming from the bound on the size of the loop propagators and discussed above, we see that globally the contribution under analysis has a dimensional gain $\gamma^{h}|h|$ with respect to the bounds described in Section 2.8, that is the desired gain.

We proceed keeping in mind the procedure followed in the proof of (2.103) and by comparison we shall show how to get the dimensional gain. Using the definitions introduced in Section 2.8 after (2.110) we can rewrite (3.20) as

$$
\begin{aligned}
& \sum_{\Omega_{\underline{v}_{0}} \backslash \Omega_{v_{0}}} \sum_{\substack{\bar{\omega}_{q} \prec \omega\left(f_{q}\right) \\
\bar{\omega}^{*} \prec \omega\left(f^{*}\right)}}\left\{\prod_{v \in \underline{v}_{0}}\left[\sum_{\cup_{w>v} \Omega_{w} \backslash \Omega_{v}} \prod_{w \geq v, w \in V_{c}} \chi\left(\tilde{\Omega}_{w, \bar{\omega}}\right) \prod_{\substack{l \in \cup_{w \geq v} T_{w} \\
\cdot}} \delta_{\omega_{l}^{+}, \omega_{l}^{-}}\right]\right. \\
&\left.\cdot \chi\left(\left\{\bar{\omega}_{1}, \bar{\omega}_{2}, \bar{\omega}_{3}, \bar{\omega}^{*}\right\}\right) \prod_{l \in T_{v_{0}}} \delta_{\omega_{l}^{+}, \omega_{l}^{-}} \prod_{q=1}^{3} \delta_{\omega_{l_{q}}^{+}, \omega_{l_{q}}^{-}}\right\} \equiv \sum_{\Omega_{\underline{v}_{0} \backslash \Omega_{v_{0}}} \sum_{\substack{\bar{\omega}_{q} \prec \omega\left(f_{q}\right) \\
\bar{\omega}^{*} \prec \omega\left(f^{*}\right)}}} \\
& {\left[\prod_{v \in \underline{v}_{0}} F_{v}\left(\tilde{\Omega}_{v, \bar{\omega}}\right)\right] \cdot \chi\left(\left\{\bar{\omega}_{1}, \bar{\omega}_{2}, \bar{\omega}_{3}, \bar{\omega}^{*}\right\}\right) \prod_{l \in T_{v_{0}}} \delta_{\omega_{l}^{+}, \omega_{l}^{-}} \prod_{q=1}^{3} \delta_{\omega_{l_{q}}^{+}, \omega_{l_{q}}^{-}} . }
\end{aligned}
$$


Defining

$$
\mathcal{F}_{\underline{v}_{0} \backslash v^{*}}\left(\tilde{\Omega}_{\underline{v}_{0} \backslash v^{*}}\right) \stackrel{\text { def }}{=} \prod_{v \in \underline{v}_{0} \backslash v^{*}} F_{v}\left(\tilde{\Omega}_{v, \bar{\omega}}\right) \prod_{l \in T_{v_{0}} \backslash l^{*}} \delta_{\omega_{l}^{+}, \omega_{l}^{-}},
$$

and calling $\Omega^{(4)}=\left\{\omega\left(f_{1}\right), \omega\left(f_{2}\right), \omega\left(f_{3}\right), \omega\left(f^{*}\right)\right\}$, we can bound (3.21) as

$$
\begin{array}{r}
(3.21) \leq \sup _{\Omega^{(4)}, \Omega_{v_{0}}}\left[\sum_{\tilde{\Omega}_{\underline{v}_{0} \backslash v^{*}}}^{* *} \mathcal{F}_{\underline{v}_{0} \backslash v^{*}}\left(\tilde{\Omega}_{\underline{v}_{0} \backslash v^{*}}\right)\right] \cdot \sup _{\Omega^{(4)}}\left[\sum_{\tilde{\Omega}_{v^{*}, \bar{\omega}}}^{*} F_{v^{*}}\left(\tilde{\Omega}_{v^{*}, \bar{\omega}}\right)\right] \\
\cdot \sum_{\bar{\omega}_{1}, \bar{\omega}_{2}, \bar{\omega}_{3}, \bar{\omega}^{*}} \chi\left(\left\{\bar{\omega}_{1}, \bar{\omega}_{2}, \bar{\omega}_{3}, \bar{\omega}^{*}\right\}\right),
\end{array}
$$

where the $* *$ on the first sum means that we are not summing over the indices in $\Omega_{v_{0}} \cup \Omega^{(4)}$, while the $*$ on the second sum means that we are not summing over the indices in $\Omega^{(4)}$. Now, the first two sums can be bounded using Lemma 2.6 and, with respect to the cases in which the two sums have just one fixed external sector (as it is the case in the proof of $(2.103)$, see (2.114)), they have a dimensional gain $\gamma^{h}$ and $\gamma^{h / 2}$ respectively. The last sum can be bounded using the following Lemma, see Appendix B for a proof.

Lemma 3.1. Given $h_{\beta} \leq h \leq 0$, the following bound holds:

$$
\sup _{\bar{\omega}_{1} \in \bar{O}_{h}} \sum_{\substack{\bar{\omega}_{q} \in \bar{O}_{h} \\ q=2,3,4}} \chi\left(\left\{\bar{\omega}_{1}, \bar{\omega}_{2}, \bar{\omega}_{3}, \bar{\omega}_{4}\right\}\right) \leq c \gamma^{-h}|h| .
$$

Using Lemma 3.1 we see that

$$
\sum_{\bar{\omega}_{1}, \bar{\omega}_{2}, \bar{\omega}_{3}, \bar{\omega}^{*}} \chi\left(\left\{\bar{\omega}_{1}, \bar{\omega}_{2}, \bar{\omega}_{3}, \bar{\omega}^{*}\right\}\right) \leq c \gamma^{-2 h}|h| .
$$

Combining all the gains and losses described above, we see that (3.20) can be bounded by the r.h.s. of (2.103) times a dimensional factor $\gamma^{-h / 2}|h|$. As discussed after (3.20) this implies that $\bar{J}_{h, n, \bar{\omega}}^{(2)}(\tau, \mathbf{P}, T ; 0, L=3)$ with $\left|P_{v_{0}}\right|=2$ admits a dimensional bound given by the r.h.s. of (3.6) with $j=0$.

(A1.2) $L=5$ : there are five extracted loop lines connecting $\tilde{P}_{v^{*}}$ with $\tilde{P}_{v_{0}} \backslash \tilde{P}_{v^{*}}$, call them $l_{1}, l_{2}, l_{3}, l_{4}, l_{5}$ and $f_{1}, f_{2}, f_{3}, f_{4}, f_{5}$ their field labels, see Fig. 4 ; the other lines exiting from $\tilde{P}_{v^{*}}$ can be contracted with any other loop line.

We replace all the modified coupling functions, all the propagators and all the Gram determinants with their absolute values. Having done this, we bound the integral over the modified coupling functions as in (2.102), the integral over the propagators of the spanning tree as in (2.101) and we bound the product of the determinants times $\prod_{q=1}^{5}\left|g_{\omega\left(f_{q}\right)}^{(h)}\left(\mathbf{x}_{l_{q}}-\mathbf{y}_{l_{q}}\right)\right|$ by the product over $v$ of the r.h.s. of (2.100). After these bounds, the sum $\sum_{\left\{i_{q} j_{q}, i_{q}^{\prime} j_{q}^{\prime}\right\}_{q=1}^{5}}^{*}$ can be bounded by $\prod_{v \in \underline{v}_{0}}\left|P_{v}\right|^{5} \leq \prod_{v \in V_{c}}\left|P_{v}\right|^{5}$, where the latter is the same product appearing in the r.h.s. of (3.6). 


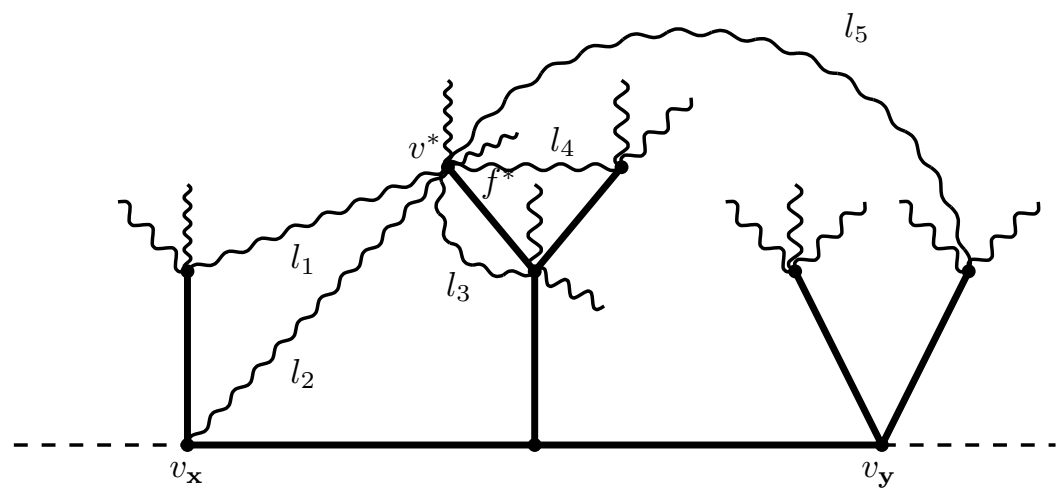

FiguRE 4. An example of case (A1.2).

We are still left with the sum over the sector indices:

$$
\sum_{\cup_{v} \Omega_{v} \backslash \Omega_{v_{0}}}\left[\prod_{\substack{v>v_{0} \\ v \in V_{c}}} \chi\left(\tilde{\Omega}_{v, \bar{\omega}}\right)\right]\left[\prod_{l \in T} \delta_{\omega_{l}^{+}, \omega_{l}^{-}}\right] \prod_{q=1}^{5} \delta_{\omega_{l_{q}}^{+}, \omega_{l_{q}}^{-}},
$$

where the product $\prod_{q=1}^{5} \delta_{\omega_{l_{q}}^{+}, \omega_{l_{q}}^{-}}$takes into account the new constraints coming from the extraction of the three loop propagators.

We want to prove that (3.26) can be bounded by the r.h.s. of (2.103) times a dimensional factor $\gamma^{h}$, that is even more than the desired dimensional gain. With the same notations as above, we rewrite (3.26) in the form

$$
\sum_{\Omega_{\underline{v}_{0}} \backslash \Omega_{v_{0}}}\left[\prod_{v \in \underline{\tilde{v}}_{0}} F_{v}\left(\tilde{\Omega}_{v, \bar{\omega}}\right)\right] \cdot \prod_{l \in T_{v_{0}}} \delta_{\omega_{l}^{+}, \omega_{l}^{-}} \prod_{q=1}^{5} \delta_{\omega_{l_{q}}^{+}, \omega_{l_{q}}^{-}} .
$$

Defining $\mathcal{F}_{\underline{v}_{0} \backslash v^{*}}\left(\tilde{\Omega}_{\underline{v}_{0} \backslash v^{*}}\right)$ as in $(3.22)$ and calling $\Omega^{(6)}=\left\{\omega\left(f_{q}\right)\right\}_{q=1}^{5} \cup\left\{\omega\left(f^{*}\right)\right\}$, we can bound (3.27) by

$$
\sup _{\Omega_{v_{0}}}\left[\sum_{\tilde{\Omega}_{\underline{v}_{0} \backslash v^{*}}}^{*} \mathcal{F}_{\underline{v}_{0} \backslash v^{*}}\left(\tilde{\Omega}_{\underline{v}_{0} \backslash v^{*}}\right)\right] \cdot \sup _{\Omega^{(6)}}\left[\sum_{\tilde{\Omega}_{v^{*}, \bar{\omega}} \backslash \Omega^{(6)}} F_{v^{*}}\left(\tilde{\Omega}_{v^{*}}\right)\right],
$$

where the $*$ on the first sum means that we are not summing over the sectors in $\Omega_{v_{0}}$. Now, the first sum can be bounded exactly as in the proof of (2.43), while the second one, using Lemma 2.6, has a dimensional gain $\gamma^{h}$, and this concludes the proof in the present case.

(A1.3) $L=1$ : there is exactly one loop line connecting $\tilde{P}_{v^{*}}$ with $\tilde{P}_{v_{0}} \backslash \tilde{P}_{v^{*}}$, call it $l_{1}$ and $f_{1}$ its field label, see Fig. 5 .

We consider the extracted loop propagator $G_{i_{1} j_{1}, i_{1}^{\prime} j_{1}^{\prime}}^{h, T_{v_{0}}}=t_{i_{1}, i_{1}^{\prime}} g_{\omega\left(f_{1}\right)}^{(h)}\left(\mathbf{x}_{l_{1}}-\mathbf{y}_{l_{1}}\right) \times$ $\delta_{\omega_{l_{1}}^{+}, \omega_{l_{1}}^{-}}$and we rewrite both $g_{\omega\left(f_{1}\right)}^{(h)}$ and the propagator of the spanning tree $g_{\omega\left(f^{*}\right)}^{(h)}$ 


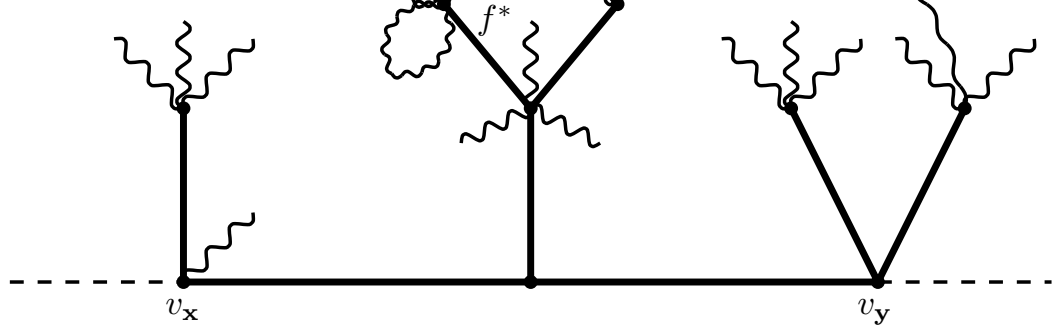

FiguRE 5. An example of case (A1.3).

as sums of isotropic propagators, to be denoted by $\bar{g}_{\bar{\omega}_{1}}^{(h)}$ and $\bar{g}_{\bar{\omega}^{*}}^{(h)}$. For any fixed configuration of internal anisotropic sectors, the new sums on the isotropic sectors can be written as:

$$
\sum_{\substack{\bar{\omega}_{1} \prec \omega\left(f_{1}\right) \\ \bar{\omega}^{*} \prec \omega\left(f^{*}\right)}} \mathbb{1}\left(\left|\bar{\omega}_{1}-\bar{\omega}^{*}\right| \leq 1\right),
$$

where the characteristic function is due to the remark that the value of the integrand in the r.h.s. of (3.15) is 0 unless $\left|\bar{\omega}_{1}-\bar{\omega}^{*}\right| \leq 1$, because of momentum conservation.

We now rewrite the determinant $\operatorname{det} \widetilde{G}^{h_{v_{0}}, T_{v_{0}}}\left(\left\{i_{1} j_{1}, i_{1}^{\prime} j_{1}^{\prime}\right\} ; 0, \mathbf{t}_{v_{0}}\right)$ as a product of two determinants, the first involving only fields in $\tilde{P}_{v^{*}} \backslash f_{1}$, the second involving only fields in $\tilde{P}_{\underline{v}_{0}} \backslash\left(\tilde{P}_{v^{*}} \cup f_{1}\right)$ :

$$
\operatorname{det} \widetilde{G}^{h, T_{v_{0}}}\left(\left\{i_{1} j_{1}, i_{1}^{\prime} j_{1}^{\prime}\right\} ; 0, \mathbf{t}_{v_{0}}\right)=\operatorname{det} \widetilde{G}_{v^{*}}^{h}\left(\mathbf{t}_{v^{*}}\right) \cdot \operatorname{det} \widetilde{G}_{\underline{v}_{0} \backslash v^{*}}^{h, T_{v_{0}}}\left(\mathbf{t}_{v_{0}}\right) .
$$

The splitting (3.30) allows us to bound the resulting expression by

$$
\begin{array}{r}
\sum_{\left\{i_{1} j_{1}, i_{1}^{\prime} j_{1}^{\prime}\right\}}^{*} \sum_{\bar{\omega}_{1}, \bar{\omega}^{*}} \mathbb{1}\left(\left|\bar{\omega}_{1}-\bar{\omega}^{*}\right| \leq 1\right)\left[v^{*}\right] \cdot\left[\underline{v}_{0} \backslash v^{*}\right] \cdot \sup _{\mathbf{x}}\left|\bar{g}_{\bar{\omega}_{1}}^{(h)}(\mathbf{x})\right| \int d \mathbf{x}\left|\bar{g}_{\bar{\omega}^{*}}^{(h)}(\mathbf{x})\right| \\
\leq c \gamma^{2 h} \gamma^{-h} \sum_{\left\{i_{1} j_{1}, i_{1}^{\prime} j_{1}^{\prime}\right\}}^{*} \sum_{\bar{\omega}_{1}, \bar{\omega}^{*}} \mathbb{1}\left(\left|\bar{\omega}_{1}-\bar{\omega}^{*}\right| \leq 1\right)\left[v^{*}\right] \cdot\left[\underline{v}_{0} \backslash v^{*}\right],
\end{array}
$$

where $\left[v^{*}\right]$ collects all the spanning tree propagators, the determinants and the endpoints associated with the vertices $v \geq v^{*}$, except the propagators of $l_{1}$ and $l^{*}$, while $\left[\underline{v}_{0} \backslash v^{*}\right]$ collects all the other terms, again except the propagators of $l_{1}$ and $l^{*}$.

Note that $\left[v^{*}\right]$ has the structure of a contribution to the effective potential with two external fields whose isotropic sectors are fixed. If we denote by $\tau^{\prime}$ the subtree of $\tau$ rooted in $v_{0}$ and containing $v^{*}$ and by $\mathbf{P}^{\prime}, \Omega^{\prime}, T^{\prime}$ the subsets of $\mathbf{P}, \Omega, T$ 
corresponding to the subtree $\tau^{\prime}$ of $\tau$, we can write

$$
\begin{aligned}
& {\left[v^{*}\right]=} \sum_{\Omega^{\prime} \backslash\left(\bar{\omega}_{1} \cup \bar{\omega}^{*}\right)}\left[\prod_{v \in \tau^{\prime}} \chi\left(\tilde{\Omega}_{v, \bar{\omega}_{1}, \bar{\omega}^{*}}\right)\right] \\
& \cdot\left|\int d\left(\mathbf{x}_{v^{*}} \backslash \mathbf{x}^{*}\right) \overline{\mathfrak{F}}_{2, h,\left(\bar{\omega}_{1}, \bar{\omega}^{*}\right)} *\left[\operatorname{det} \widetilde{G}_{v^{*}}^{h}\left(\mathbf{t}_{v^{*}}\right) \cdot W_{\tau^{\prime}, \mathbf{P}^{\prime}, \Omega^{\prime}, T^{\prime}}^{(\bmod )}\left(\mathbf{x}_{v^{*}}\right)\right]\right|,
\end{aligned}
$$

where $\mathbf{x}^{*}$ is the space time point of $\mathbf{x}_{v^{*}}$ where the line $l^{*}$ is hooked on and, in analogy to (3.16), we defined

$$
\tilde{\Omega}_{v, \bar{\omega}_{1}, \bar{\omega}^{*}}=\left\{\omega(f) \in O_{h(f)}, f \in P_{v} \backslash\left\{f_{1}, f^{*}\right\}\right\} \cup\left\{\bar{\omega}_{f_{1}}=\bar{\omega}_{1}, \bar{\omega}_{f^{*}}=\bar{\omega}^{*}\right\} .
$$

Note that, in the case that $v^{*}$ is an endpoint, the r.h.s. of (3.32) can be bounded by (3.8), with $k=h^{\prime}=h$ and $j=0$, which has a dimensional factor $\gamma^{h}|h|$ more than the dimensional estimate one would get simply using (2.92) and Lemma 2.2. If $v^{*}$ is not an endpoint, we will prove below that

$$
\left[v^{*}\right] \leq(c|U|)^{m_{4}\left(v^{*}\right)} \gamma^{2 h}|h| \prod_{v \in V_{c}, v>v^{*}} \frac{1}{s_{v} !}\left|P_{v}\right|^{5} \gamma^{\delta\left(\left|P_{v}\right|\right)\left(h_{v}-h_{v^{\prime}}\right)},
$$

where $m_{4}\left(v^{*}\right)$ is the number of endpoints of $\tau^{\prime}$ and $v^{\prime}$ is the $c$-vertex immediately preceding $v$ on $\tau^{\prime}$. The proof of (3.34) will be postponed, see item (B) below. Note that the bound in (3.34) has a $\gamma^{h}|h|$ more than the dimensional bound one would get by repeating the proof of Theorem 2.1 .

Let us now consider the factor $\left[\underline{v}_{0} \backslash v^{*}\right]$. It can be written in the following way:

$$
\left[\underline{v}_{0} \backslash v^{*}\right]=\int d \mathbf{x}_{1} d \mathbf{x}_{2} d \mathbf{y}\left|G_{\bar{\omega}, \bar{\omega}_{1}, \bar{\omega}^{*}}^{(4)}\left(\mathbf{x}, \mathbf{y}, \mathbf{x}_{1}, \mathbf{x}_{2}\right)\right|
$$

where $\mathbf{x}_{1}$ and $\mathbf{x}_{2}$ are the space-time points in $\tau \backslash \tau^{\prime}$, where the two propagators of $l^{*}$ and $l_{1}$ are hooked on. Note that $G_{\bar{\omega}, \bar{\omega}_{1}, \bar{\omega}^{*}}^{(4)}\left(\mathbf{x}, \mathbf{y}, \mathbf{x}_{1}, \mathbf{x}_{2}\right)$ has the same structure of a contribution to the effective potential with four external fields with fixed isotropic sectors. It follows that

$$
\left[\underline{v}_{0} \backslash v^{*}\right] \leq \tilde{G}_{\omega, \omega_{1}, \omega^{*}}^{(4)},
$$

where $\omega, \omega_{1}, \omega_{2}$ are the anisotropic sectors containing $\bar{\omega}, \bar{\omega}_{1}, \bar{\omega}^{*}$, respectively and $\tilde{G}_{\omega, \omega_{1}, \omega^{*}}^{(4)}$ has the same structure of $J_{h, n-m_{4}\left(v^{*}\right)}^{(4)}\left(\tau \backslash \tau^{\prime}, \mathbf{P} \backslash \mathbf{P}^{\prime}, T \backslash T^{\prime}\right)$, see (2.96).

By using Lemma 2.7, we get

$$
\tilde{G}_{\omega, \omega_{1}, \omega^{*}}^{(4)} \leq(c|U|)^{n-m_{4}\left(v^{*}\right)} \prod_{v \in V_{c}, v \notin \tau^{\prime}, v>v_{0}} \gamma^{\delta\left(\left|P_{v}\right|\right)\left(h_{v}-h_{v^{\prime}}\right)} .
$$

In order to complete the proof, we still have to bound the two sums in (3.31); the first one gives a factor $\prod_{v \in \underline{v}_{0}}\left|P_{v}\right| \leq \prod_{v \in V_{c}, v \notin \tau^{\prime}}\left|P_{v}\right|$, the second a factor $\gamma^{-h}$. Combining all these bounds, we find that also the term with $L=1$ can be bounded by the r.h.s. of (3.6). 


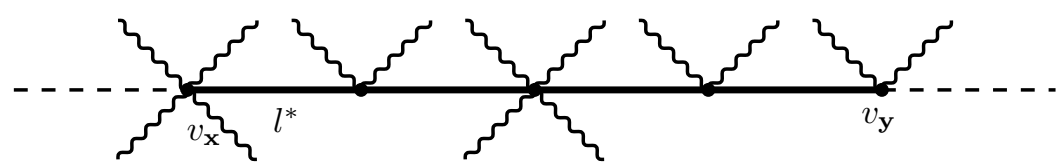

Figure 6. An example of case (A2).

(A2) $s_{v_{0}} \geq 2$ and $T_{v_{0}} \equiv l_{\mathbf{x}, \mathbf{y}}$. In this case by hypothesis all the vertices in $\underline{v}_{0}$ belong to $l_{\mathbf{x}, \mathbf{y}}$ and each of them has at least two free loop lines contracted into the Gram determinant, see Fig. 6.

We consider the vertex $v_{\mathbf{x}}$ and we note that, by construction, $v_{\mathbf{x}}$ has one external line coinciding with $f_{\mathbf{x}}$ and another one belonging to the spanning tree $T_{v_{0}}$, let us call it $f^{*}$. Calling $\tilde{P}_{\underline{v}_{0}}$ the set of loop lines contracted into the Gram determinant and $\tilde{P}_{v_{\mathbf{x}}}=\tilde{P}_{\underline{v}_{0}} \cap P_{v_{\mathbf{x}}}$, we again write $G^{h, T_{v_{0}}}$ in blocks, as in (3.10), with $A$ the block with both row and column indices in $\tilde{P}_{v_{x}}, B$ the block with row indices in $\tilde{P}_{v_{\mathrm{x}}}$ and column indices in $\tilde{P}_{\underline{v}_{0}} \backslash \tilde{P}_{v_{\mathrm{x}}}, C$ the block with row indices in $\tilde{P}_{\underline{v}_{0}} \backslash \tilde{P}_{v_{\mathrm{x}}}$ and column indices in $\tilde{P}_{v_{\mathrm{x}}}$ and $D$ the block with both row and column indices in $\tilde{P}_{\underline{v}_{0}} \backslash \tilde{P}_{v_{\mathbf{x}}}$.

In this case, by a fourth order expansion of $\left(\begin{array}{cc}A & B \\ C & D\end{array}\right)$ around $\left(\begin{array}{cc}A & 0 \\ 0 & D\end{array}\right)$, we get the analogue of (3.13) and, since $\left|P_{v_{0}}\right|=2$, we get the bound analogue to (3.14):

$$
\bar{J}_{h, n, \bar{\omega}}^{(2)}(\tau, \mathbf{P}, T ; 0) \leq \sum_{L=0,2,4} \bar{J}_{h, n, \bar{\omega}}^{(2)}(\tau, \mathbf{P}, T ; 0, L)
$$

where $\bar{J}_{h, n, \bar{\omega}}^{(2)}(\tau, \mathbf{P}, T ; 0, L)$ with $L=0,2,4$ are defined by an equation completely analogous to (3.15), where, if $L=2,4$, the sum $\sum_{\left\{i_{q} j_{q}, i_{q_{j}^{\prime}}^{*} j_{q}^{\prime}\right\}_{q=1}^{L}}$ has to be interpreted as the sum over the indices $\left\{i_{q} j_{q}, i_{q}^{\prime} j_{q}^{\prime}\right\}_{q=1}^{L}$ such that $f_{i_{q} j_{q}} \in P_{v_{\times}}$and $f_{i_{q}^{\prime} j_{q}^{\prime}} \in P_{\underline{v}_{0}} \backslash$ $P_{v_{\mathbf{x}}}$ or vice versa, while, if $L=0$, the factor $\frac{1}{L !} \sum_{\left\{i_{q} j_{q}, i_{q}^{\prime} j_{q}^{\prime}\right\}_{q=1}^{L}}^{*}\left[\prod_{q=1}^{L} G_{i_{q} j_{q}, i_{q}^{\prime} j_{q}^{\prime}}^{h, T_{v_{0}}}\right]$. $\operatorname{det} \widetilde{G}^{h, T_{v_{0}}}\left(\left\{i_{q} j_{q}, i_{q}^{\prime} j_{q}^{\prime}\right\}_{q=1}^{L} ; s_{L}, \mathbf{t}_{v_{0}}\right)$ in (3.15) must be interpreted as equal to $\operatorname{det} A$. $\operatorname{det} D$. In other words, the bound (3.38) allows us to distinguish between the contributions to $\bar{J}_{h, n, \bar{\omega}}^{(2)}(\tau, \mathbf{P}, T ; 0)$ such that: $v_{\mathbf{x}}$ is connected with $\underline{v}_{0} \backslash v_{\mathbf{x}}$ only by a line of the spanning tree $(L=0)$ or $v_{\mathbf{x}}$ is connected with $\underline{v}_{0} \backslash v_{\mathbf{x}}$ also by exactly two loop lines $(L=2)$ or $v_{\mathbf{x}}$ is connected with $\underline{v}_{0} \backslash v_{\mathbf{x}}$ also by four or more loop lines $(L=4)$.

We now want to show how to get the desired dimensional gain for the three contributions $\bar{J}_{h, n, \bar{\omega}}^{(2)}(\tau, \mathbf{P}, T ; 0, L), L=0,2,4$, in the r.h.s. of (3.38).

(A2.1) $L=2$ : there are exactly two loop lines connecting $\tilde{P}_{v_{\mathrm{x}}}$ with $\tilde{P}_{v_{0}} \backslash \tilde{P}_{v_{x}}$, call them $l_{1}, l_{2}$ and $f_{1}, f_{2}$ their field labels, see Fig. 7 . 


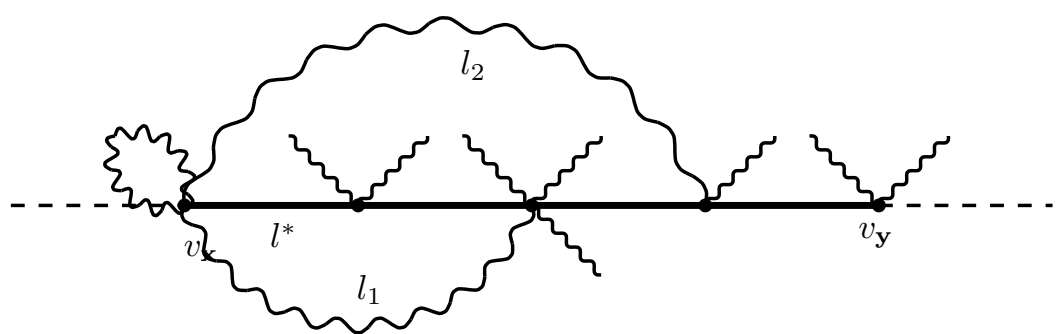

Figure 7. An example of case (A2.1).

This case can be treated in a way similar to the case (A1.1) above. Let us consider the product $\prod_{q=1}^{2} G_{i_{q} j_{q}, i_{q}^{\prime} j_{q}^{\prime}}^{h, T_{v_{0}}}=\prod_{q=1}^{2} t_{i_{q}, i_{q}^{\prime}} g_{\omega\left(f_{q}\right)}^{(h)}\left(\mathbf{x}_{l_{q}}-\mathbf{y}_{l_{q}}\right) \delta_{\omega_{l_{q}}^{+}, \omega_{l_{q}}^{-}}$in $(3.15)$ and let us rewrite both the extracted loop propagators $g_{\omega\left(f_{q}\right)}^{(h)}$ with $q=1,2$ and the propagator of the spanning tree $g_{\omega\left(f^{*}\right)}^{(h)}$ (we recall that $f^{*}$ is the line of $T_{v_{0}}$ belonging to $\left.P_{v_{\mathbf{x}}}\right)$ as sums of isotropic propagators, to be denoted by $\bar{g}_{\bar{\omega}_{q}}^{(h)}, q=1,2$, and $\bar{g}_{\bar{\omega}^{*}}^{(h)}$. For any fixed configuration of internal anisotropic sectors, the new sums on the isotropic sectors can be written as

$$
\sum_{\bar{\omega}_{q} \prec \omega\left(f_{q}\right)} \sum_{\bar{\omega}^{*} \prec \omega\left(f^{*}\right)},
$$

where the meaning of the symbol $\prec$ is the same as in (2.108). Note also that, by construction, the summand in the r.h.s. of (3.15) is 0 unless $\chi\left(\left\{\bar{\omega}, \bar{\omega}_{1}, \bar{\omega}_{2}, \bar{\omega}^{*}\right\}\right)=1$, so that we can freely multiply the summand in the r.h.s. of $(3.15)$ by $\chi\left(\left\{\bar{\omega}, \bar{\omega}_{1}\right.\right.$, $\left.\left.\bar{\omega}_{2}, \bar{\omega}^{*}\right\}\right)$. In this case it is crucial the assumption that the external sector index corresponding to $f_{\mathbf{x}}$ is isotropic.

Having done this, we can bound the resulting expression by replacing all the modified coupling functions, all the propagators and all the Gram determinants with their absolute values. Now, we can bound the integral over the absolute values of the modified coupling functions as in (2.102) (by using again (3.18) in place of (2.92)), the integral over the absolute values of the propagators of the spanning tree as in (2.101) and the product of the determinants times $\prod_{q=1}^{2}\left|\bar{g}_{\bar{\omega}\left(f_{q}\right)}^{(h)}\left(\mathbf{x}_{l_{q}}-\mathbf{y}_{l_{q}}\right)\right|$ by the product over $v$ of the r.h.s. of (2.100) times a dimensional gain $\gamma^{h}$. After these bounds, the sum $\sum_{\left\{i_{q} j_{q}, i_{q}^{\prime} j_{q}^{\prime}\right\}_{q=1}^{2}}^{*}$ can be bounded by $\prod_{v \in \underline{v}_{0}}\left|P_{v}\right|^{2} \leq \prod_{v \in V_{c}}\left|P_{v}\right|^{5}$ (the latter is the same product appearing in the r.h.s. of (3.6)) and we are still left with the sum over the sector indices:

$$
\sum_{\substack{\Omega_{v} \backslash \Omega_{v_{0}} \\ \bar{c}_{\bar{\omega}_{q}} \prec \omega\left(f_{q}\right) \\ \bar{\omega}^{*} \prec \omega\left(f^{*}\right)}}\left[\prod_{\substack{v>v_{0} \\ v \in V_{c}}} \chi\left(\tilde{\Omega}_{v, \bar{\omega}}\right)\right] \chi\left(\left\{\bar{\omega}, \bar{\omega}_{1}, \bar{\omega}_{2}, \bar{\omega}^{*}\right\}\right)\left[\prod_{l \in T} \delta_{\omega_{l}^{+}, \omega_{l}^{-}}\right] \prod_{q=1}^{2} \delta_{\omega_{l_{q}}^{+}, \omega_{l_{q}}^{-}} .
$$


where the product $\chi\left(\left\{\bar{\omega}, \bar{\omega}_{1}, \bar{\omega}_{2}, \bar{\omega}^{*}\right\}\right) \prod_{q=1}^{2} \delta_{\omega_{l_{q}}^{+}, \omega_{l_{q}}^{-}}$takes into account the new constraints coming from the extraction of the two loop propagators.

We want to prove that (3.40) can be bounded by the r.h.s. of (2.103) times a dimensional factor $|h|$. Combining this loss with the gain $\gamma^{h}$ coming from the bound on the size of the loop propagators and discussed above, we see that globally the contribution under analysis has a dimensional gain $\gamma^{h}|h|$ with respect to the bounds described in Section 2.8, that is the desired gain.

We proceed as in item (A1.1) above. With the same notations as in (3.21), we can rewrite $(3.40)$ as

$$
\sum_{\Omega_{\underline{v}_{0}} \backslash \Omega_{v_{0}}} \sum_{\substack{\bar{\omega}_{q} \prec \omega\left(f_{q}\right) \\ \bar{\omega}^{*} \prec \omega\left(f^{*}\right)}}\left[\prod_{v \in \underline{\underline{v}}_{0}} F_{v}\left(\tilde{\Omega}_{v, \bar{\omega}}\right)\right] \cdot \chi\left(\left\{\bar{\omega}, \bar{\omega}_{1}, \bar{\omega}_{2}, \bar{\omega}^{*}\right\}\right) \prod_{l \in T_{v_{0}}} \delta_{\omega_{l}^{+}, \omega_{l}^{-}} \prod_{q=1}^{2} \delta_{\omega_{l_{q}}^{+}, \omega_{l_{q}}^{-}} .
$$

Defining

$$
\mathcal{F}_{\underline{v}_{0} \backslash v_{\mathbf{x}}}\left(\tilde{\Omega}_{\underline{v}_{0} \backslash v_{\mathbf{x}}}\right) \stackrel{\text { def }}{=} \prod_{v \in \underline{v}_{0} \backslash v_{\mathbf{x}}} F_{v}\left(\tilde{\Omega}_{v, \bar{\omega}}\right) \prod_{l \in T_{v_{0}} \backslash l^{*}} \delta_{\omega_{l}^{+}, \omega_{l}^{-}},
$$

and calling $\Omega^{(3)}=\left\{\omega\left(f_{1}\right), \omega\left(f_{2}\right), \omega\left(f^{*}\right)\right\}$, we can bound (3.41) by

$$
\begin{array}{r}
\sup _{\Omega^{(3)}}\left[\sum_{\tilde{\Omega}_{\underline{v}_{0} \backslash v_{\mathbf{x}}}}^{*} \mathcal{F}_{\underline{v}_{0} \backslash v_{\mathbf{x}}}\left(\tilde{\Omega}_{\underline{v}_{0} \backslash v^{*}}\right)\right] \cdot \sup _{\bar{\omega}, \Omega^{(3)}}\left[\sum_{\tilde{\Omega}_{v_{\mathbf{x}}, \bar{\omega}}}^{*} F_{v^{*}}\left(\tilde{\Omega}_{v_{\mathbf{x}}, \bar{\omega}}\right)\right] \\
\cdot \sum_{\bar{\omega}_{1}, \bar{\omega}_{2}, \bar{\omega}^{*}} \chi\left(\left\{\bar{\omega}, \bar{\omega}_{1}, \bar{\omega}_{2}, \bar{\omega}^{*}\right\}\right),
\end{array}
$$

where the $*$ on the sums means that we are not summing over the indices in $\{\bar{\omega}\} \cup \Omega^{(3)}$. Now, the first two sums can be bounded using Lemma 2.6 and, with respect to the cases in which the two sums have just one fixed external sector (as it is the case in the proof of (2.103), see (2.114)), they both have a dimensional gain $\gamma^{h / 2}$ (so combining the two, their product has a gain of $\gamma^{h}$ ). By Lemma 3.1 we see that the contribution from the last sum can be bounded by $c \gamma^{-h}|h|$, and we see that (3.40) can be bounded by the r.h.s. of (2.103) times a dimensional factor $|h|$. As discussed after (3.40) this implies that $\bar{J}_{h, n, \bar{\omega}}^{(2)}(\tau, \mathbf{P}, T ; 0, L=2)$ with $\left|P_{v_{0}}\right|=2$ admits a dimensional bound given by the r.h.s. of (3.6) with $j=0$.

(A2.2) $L=4$ : there are four extracted loop lines connecting $\tilde{P}_{v_{\mathbf{x}}}$ with $\tilde{P}_{\underline{v}_{0}} \backslash \tilde{P}_{v_{\mathbf{x}}}$, call them $l_{1}, l_{2}, l_{3}, l_{4}$ and $f_{1}, f_{2}, f_{3}, f_{4}$ their field labels, see Fig. 8 .

This case can be treated in a way similar to item (A1.2) above: we replace all the modified coupling functions, all the propagators and all the Gram determinants with their absolute values. Having done this, we bound the integral over the modified coupling functions as in (2.102), the integral over the propagators of the spanning tree as in (2.101) and we bound the product of the determinants times $\prod_{q=1}^{4}\left|g_{\omega\left(f_{q}\right)}^{(h)}\left(\mathbf{x}_{l_{q}}-\mathbf{y}_{l_{q}}\right)\right|$ by the product over $v$ of the r.h.s. of (2.100). After these 


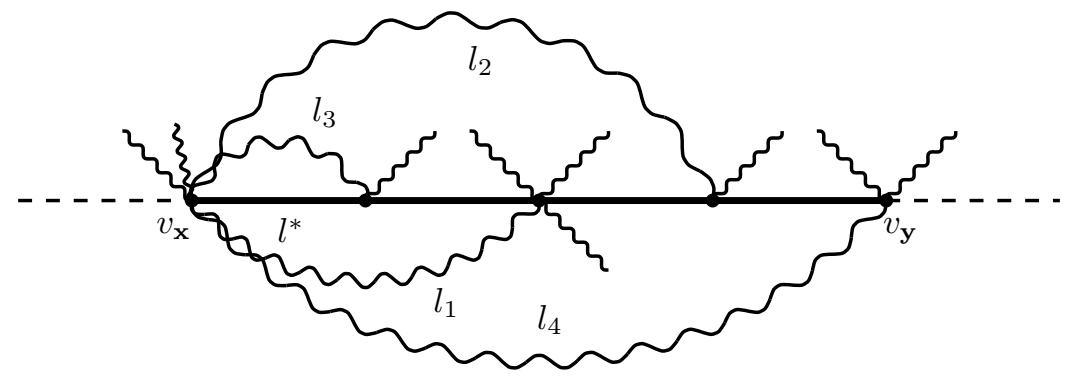

Figure 8. An example of case (A2.2).

bounds, the sum $\sum_{\left\{i_{q} j_{q}, i_{q}^{\prime} j_{q}^{\prime}\right\}_{q=1}^{4}}^{*}$ can be bounded by $\prod_{v \in \underline{v}_{0}}\left|P_{v}\right|^{4} \leq \prod_{v \in V_{c}}\left|P_{v}\right|^{5}$ (the latter is the same product appearing in the r.h.s. of (3.6)) and we are still left with the sum over the sector indices:

$$
\sum_{\cup \Omega_{v} \backslash \Omega_{v_{0}}}\left[\prod_{\substack{v>v_{0} \\ v \in V_{c}}} \chi\left(\tilde{\Omega}_{v, \bar{\omega}}\right)\right]\left[\prod_{l \in T} \delta_{\omega_{l}^{+}, \omega_{l}^{-}}\right] \prod_{q=1}^{4} \delta_{\omega_{l_{q}}^{+}, \omega_{l_{q}}^{-}},
$$

where the product $\prod_{q=1}^{4} \delta_{\omega_{l_{q}}^{+}, \omega_{l_{q}}^{-}}$takes into account the new constraints coming from the extraction of the three loop propagators.

We want to prove that (3.44) can be bounded by the r.h.s. of (2.103) times a dimensional factor $\gamma^{h}$, that is even more than the desired dimensional gain. With the same notations as above, we rewrite (3.44) in the form

$$
\sum_{\Omega_{\underline{v}_{0}} \backslash \Omega_{v_{0}}}\left[\prod_{v \in \underline{\underline{v}}_{0}} F_{v}\left(\tilde{\Omega}_{v, \bar{\omega}}\right)\right] \cdot \prod_{l \in T_{v_{0}}} \delta_{\omega_{l}^{+}, \omega_{l}^{-}} \prod_{q=1}^{4} \delta_{\omega_{l q}^{+}, \omega_{l_{q}}^{-}} .
$$

Defining $\mathcal{F}_{\underline{v}_{0} \backslash v_{\mathbf{x}}}\left(\tilde{\Omega}_{\underline{v}_{0} \backslash v_{\mathbf{x}}}\right)$ as in (3.42) and calling $\Omega^{(5)}=\left\{\omega\left(f_{q}\right)\right\}_{q=1}^{4}, \cup\left\{\omega\left(f^{*}\right)\right\}$, we can bound (3.45) by

$$
\sup _{\bar{\omega}}\left[\sum_{\tilde{\Omega}_{\underline{v}_{0} \backslash v_{\mathbf{x}}}}^{*} \mathcal{F}_{\underline{v}_{0} \backslash v_{\mathbf{x}}}\left(\tilde{\Omega}_{\underline{v}_{0} \backslash v_{\mathbf{x}}}\right)\right] \cdot \sup _{\bar{\omega}, \Omega^{(5)}}\left[\sum_{\tilde{\Omega}_{v_{\mathbf{x}}, \bar{\omega} \backslash \Omega^{(5)}}}^{*} F_{v_{\mathbf{x}}}\left(\tilde{\Omega}_{v_{\mathbf{x}}}\right)\right],
$$

where the $*$ on the sums means that we are not summing over the sectors in $\Omega_{v_{0}}$. Now, the first sum can be bounded exactly as in the proof of (2.43), while the second one, using Lemma 2.6, has a dimensional gain $\gamma^{h}$, and this concludes the proof in the present case.

(A2.3) $L=0$ : there are no loop lines connecting $\tilde{P}_{v_{\mathbf{x}}}$ with $\tilde{P}_{v_{0}} \backslash \tilde{P}_{v_{\mathbf{x}}}$, see Fig. 9 .

We consider the line $l^{*}$ in $T_{v_{0}}$ anchored to $v_{\mathbf{x}}$ and we rewrite the corresponding propagator $g_{\omega\left(f^{*}\right)}^{(h)}$ as a sum of isotropic propagators, to be denoted by $\bar{g}_{\bar{\omega}^{*}}^{(h)}$. For any fixed configuration of internal anisotropic sectors, the new sum on the isotropic sectors can be written as $\sum_{\bar{\omega}^{*} \prec \omega\left(f^{*}\right)} \mathbb{1}\left(\left|\bar{\omega}-\bar{\omega}^{*}\right| \leq 1\right)$, where 


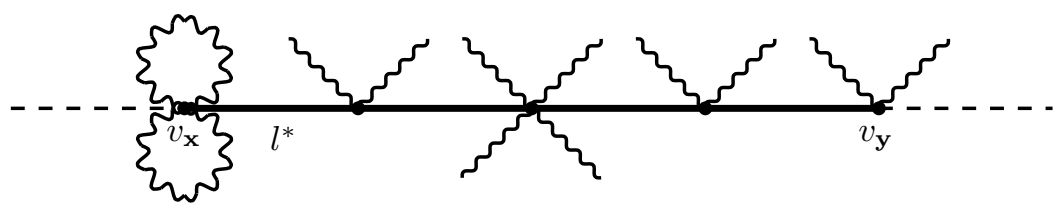

Figure 9. An example of case (A2.3).

the characteristic function is due to the remark that the value of the integrand in the r.h.s. of (3.15) is 0 unless $\left|\bar{\omega}-\bar{\omega}^{*}\right| \leq 1$. We recall that if $L=0$ the factor $\frac{1}{L !} \sum_{\left\{i_{q} j_{q}, i_{q}^{\prime} j_{q}^{\prime}\right\}_{q=1}^{L}}^{*}\left[\prod_{q=1}^{L} G_{i_{q} j_{q}, i_{q}^{\prime} j_{q}^{\prime}}^{h, T_{v^{\prime}}}\right] \cdot \operatorname{det} \widetilde{G}^{h, T_{v_{0}}}\left(\left\{i_{q} j_{q}, i_{q}^{\prime} j_{q}^{\prime}\right\}_{q=1}^{L} ; s_{L}, \mathbf{t}_{v_{0}}\right)$ in (3.15) must be interpreted as equal to $\operatorname{det} A \cdot \operatorname{det} D$. If we rename the matrix $A$ by $\widetilde{G}_{v_{\mathbf{x}}}^{h}\left(\mathbf{t}_{v_{\mathbf{x}}}\right)$ and the matrix $D$ by $\widetilde{G}_{\underline{v}_{0} \backslash v_{\mathbf{x}}}^{h, T_{v_{0}}}\left(\mathbf{t}_{v_{0}}\right)$, we can bound $\bar{J}_{h, n, \bar{\omega}}^{(2)}(\tau, \mathbf{P}, T ; 0,0)$ in a way similar to (3.31):

$$
\sum_{\bar{\omega}^{*}} \mathbb{1}\left(\left|\bar{\omega}-\bar{\omega}^{*}\right| \leq 1\right)\left[v_{\mathbf{x}}\right] \cdot\left[\underline{v}_{0} \backslash v_{\mathbf{x}}\right] \int d \mathbf{x}\left|\bar{g}_{\bar{\omega}^{*}}^{(h)}(\mathbf{x})\right| .
$$

Note that $\left[v_{\mathbf{x}}\right]$ has a form similar to (3.32), so that it can be bounded by using (3.8), if $v_{\mathbf{x}}$ is an endpoint, or (3.34), in the opposite case; this will produce a gain $\gamma^{h}|h|$ with respect to the dimensional estimate one would get simply using (2.92) and Lemma 2.2.

Let us now consider $\left[\underline{v}_{0} \backslash v_{\mathbf{x}}\right]$. It has the structure of a contribution to the effective potential with two external fields with fixed isotropic sectors, which we substitute in the bound with the corresponding anisotropic ones, before using Theorem 2.1. Hence no gain or loss follows from this operation, as well from the bound $\int d \mathbf{x}\left|\bar{g}_{\bar{\omega}^{*}}^{(h)}\right| \leq c \gamma^{-h}$. Since $\sum_{\bar{\omega}^{*}} \mathbb{1}\left(\left|\bar{\omega}-\bar{\omega}^{*}\right| \leq 1\right) \leq c$, the r.h.s. of $(3.47)$ can be bounded by $(C|U|)^{n}\left(|h| \gamma^{2 h}\right) \gamma^{h} \gamma^{-h}=(C|U|)^{n}|h| \gamma^{2 h}$, times the usual factor $\prod_{v \in V_{c}, v>v_{\mathbf{x}}} \frac{1}{s_{v} !}\left|P_{v}\right|^{5} \gamma^{\delta\left(\left|P_{v}\right|\right)\left(h_{v}-h_{v^{\prime}}\right)}$.

We now turn to the proof of the case $s_{v_{0}}=1$ and of the bound (3.34).

(B) In this item we prove the dimensional gain for the case $s_{v_{0}}=1$. The proof includes the unfinished proofs of items (A1.3) and (A2.3) above, that is the proofs of the bounds (3.34) and of the analogue bound for $\left[v_{\mathbf{x}}\right]$ in (A2.3).

If $s_{v_{0}}=1$, we call $v_{m}$ the first non trivial vertex following $v_{0}$ on $\tau$ and $v_{1}, \ldots, v_{m-1}$ the trivial $c$-vertices preceding $v_{m}$ on $\tau$, see Fig. 10 , where the $c$ vertices are represented as dots of larger size with respect to the others.

Let us call $k=h_{v_{m}}$ the scale of $v_{m}$ and $\underline{v}_{m}$ the set of $c$-vertices immediately following $v_{m}$ on $\tau$; we shall also call tadpole propagator any propagator linking two external fields of a single vertex. Very roughly, the strategy will consist in getting a first dimensional gain $\gamma^{k}|k|$ on scale $k=h_{v_{m}}$ by a loop extraction similar to that described in Section 3.1, by distinguishing again among several cases. Moreover, in order to "transfer the gain on scale $h=h_{v_{0}}$ ", we shall suitably "extract from 


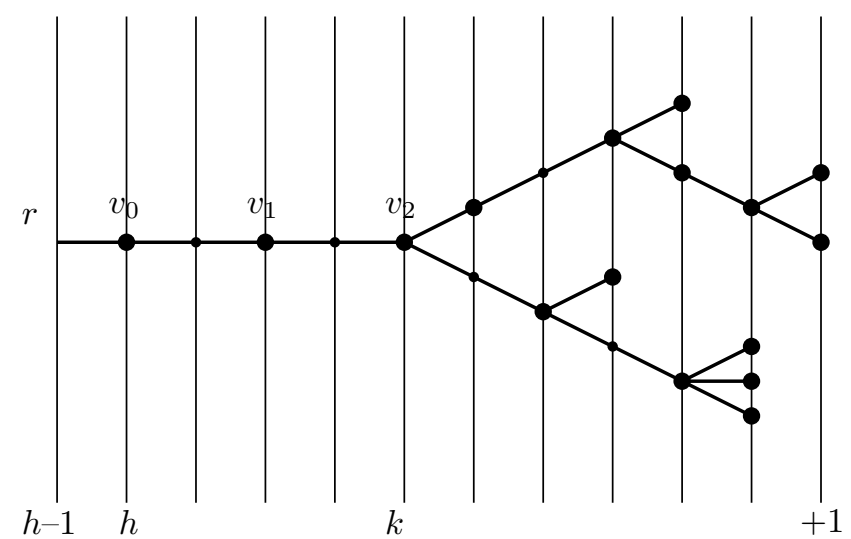

Figure 10. An example of tree in case (B).

the determinant" one tadpole propagator of scale $h$ and expand it into a sum of isotropic propagators, as described below.

Calling $\operatorname{det} G_{v_{0}}^{h}, \operatorname{det} G_{v_{1}}^{h_{v_{1}}}, \ldots, \operatorname{det} G_{v_{m-1}}^{h_{v_{m-1}}}$ the determinants of the tadpole propagators contracted on scales $h, h_{v_{1}}, \ldots, h_{v_{m-1}}$ respectively, we want to prove the following:

$$
\begin{aligned}
& \sum_{\Omega \backslash \Omega_{v_{0}}}\left[\prod_{v \in \tau} \chi\left(\tilde{\Omega}_{v, \bar{\omega}}\right)\right] \mid \int d\left(\mathbf{x}_{v_{m}} \backslash \mathbf{x}^{*}\right) \overline{\mathfrak{F}}_{2, h,(\bar{\omega}, \bar{\omega})} *\left[\left(\prod_{i=0}^{m-1} \operatorname{det} G_{v_{i}}^{h_{v_{i}}}\right)\right. \\
& \left.\cdot W_{\tau^{\prime}, \mathbf{P}^{\prime}, \Omega^{\prime}, T^{\prime}}^{(\bmod )}\left(\mathbf{x}_{v_{m}}\right)\right]\left.\left|\leq(c|U|)^{n} \gamma^{2 h}\right| h\left|\prod_{v \in V_{c}, v>v_{0}} \frac{1}{s_{v} !}\right| P_{v}\right|^{5} \gamma^{\delta\left(\left|P_{v}\right|\right)\left(h_{v}-h_{v^{\prime}}\right)}
\end{aligned}
$$

where the 1.h.s. is equal to $\bar{J}_{h, n,(\bar{\omega}, \bar{\omega})}^{(2)}(\tau, \mathbf{P}, T ; 0)$ and $\tau^{\prime}$ is the subtree of $\tau$ such that $v_{0}^{\prime}$, i.e., the first vertex following the root on $\tau^{\prime}$, coincides with $v_{m}$. Correspondingly, $\mathbf{P}^{\prime}, \Omega^{\prime}, T^{\prime}$ are the field labels, the sector labels and the spanning tree of $\tau^{\prime}$.

Comparing (3.48) with the naive bound one would get by repeating the proof of Theorem 2.1 we see that, again, we want to find a dimensional gain of $\gamma^{h}|h|$ with respect to the estimates in Sect. 2.

Remark. After a suitable (and obvious) identification of the symbols in (3.32) with those in (3.48), it becomes clear that the bound (3.34) and the analogue bound for $\left[v_{\mathbf{x}}\right]$ in item $(\mathrm{A} 2.3)$ are essentially the same as (3.48) (unless for the specific values of the external fixed isotropic sectors). Then the proof of (3.48) described in the following applies unvaried to the analysis of (3.34) and of the analogue bound for $\left[v_{\mathbf{x}}\right]$ so that the bound (3.48) will also complete the proof of the dimensional gain in items (A1.3) and (A2.3) above. 
We look into the structure of $v_{m}$. Since, by construction, $s_{v_{m}} \geq 2$, the spanning tree $T_{v_{m}}$ can be represented as in Fig. 2 or in Fig. 6 ; the only difference is that the loop fields may have different scales between $k$ and $h$, with the important constraint that at least four fields have scale $h$ (indeed two fields of scale $h$ would be sufficient). Let us choose, as before, $v_{\mathbf{x}}$ as the root of $T_{v_{m}}$ and select one leaf of $T_{v_{m}}$, to be called again $v^{*}$. If it is possible, as in the case of Fig. 2, we choose $v^{*} \neq v_{\mathbf{y}}$, otherwise (that is if $T_{v_{m}} \equiv l_{\mathbf{x}, \mathbf{y}}$, as in Fig. 6) it will be necessarily $v^{*} \equiv v_{\mathbf{y}}$. By construction $v^{*}$ has one external line belonging to the spanning tree $T_{v_{m}}$, call it $f^{*}$ and call $\omega^{*}$ its sector label. If $v^{*} \neq v_{\mathbf{y}}, P_{v^{*}}$ will have an odd number of fields associated to loop lines contracted into the Gram determinant. If $v^{*}=v_{\mathbf{y}}, P_{v^{*}}$ will have an even number of fields associated to loop lines contracted into the Gram determinant and one more field belonging to $P_{v_{0}}$ (associated to a fixed isotropic sector index).

We consider the product of determinants

$$
\left(\prod_{i=0}^{m-1} \operatorname{det} G_{v_{i}}^{h_{v_{i}}}\right) \cdot \operatorname{det} G^{h_{v_{m}}, T_{v_{m}}},
$$

where $\operatorname{det} G^{h_{v_{m}}, T_{v_{m}}}$ is the determinant of the loop lines contracted on scale $h_{v_{m}}$, if this family of lines is not empty, otherwise it is equal to 1 . The product in (3.49) can be rewritten as the determinant of a single "big" matrix $G_{\underline{v}_{m}}$ of all the loop lines contracted on scales $h, h_{v_{1}}, \ldots, h_{v_{m}}$ (all the loop lines in the examples similar to those of Fig. 2 and 6 ). Let us call $\tilde{P}_{v^{*}} \subset P_{v^{*}}$ the set of loop lines of $P_{v^{*}}$ contracted into $G_{\underline{v}_{m}}$ and $\tilde{P}_{\underline{v}_{m}}$ the whole set of loop lines in $\cup_{w \in \underline{v}_{m}} P_{w}$ contracted into $G_{\underline{v}_{m}}$. In analogy with the procedure described in item (A1), we rewrite $G_{\underline{v}_{m}}$ in blocks, as in the r.h.s. of (3.10), with the blocks $A, B, C, D$ defined in a way similar to that introduced after (3.10): that is $A$ is the block with both row and column indices in $\tilde{P}_{v^{*}}, B$ is the block with row indices in $\tilde{P}_{v^{*}}$ and column indices in $\tilde{P}_{\underline{v}_{m}} \backslash \tilde{P}_{v^{*}}$, and so on. Then we Taylor expand the $\operatorname{determinant} \operatorname{det}\left(\begin{array}{ll}A & B \\ C & D\end{array}\right)$ around $\operatorname{det}\left(\begin{array}{cc}A & 0 \\ 0 & D\end{array}\right)$ up to fourth or fifth order, depending whether $v^{*}$ is equal to $v_{\mathbf{y}}$ or not: in this way we can distinguish the contributions to (3.48) in which there are exactly $L=0,1,2,3$ loop lines connecting $v^{*}$ with $\underline{v}_{m} \backslash v^{*}$ from those with at least 4 or 5 loop lines, depending on whether $v^{*}$ is equal to $v_{\mathbf{y}}$ or not. We do not write explicitly here the expressions corresponding to these various contributions: they are similar to the r.h.s. of (3.15) with $\widetilde{G}^{h, T_{v_{0}}}$ replaced by a suitable minor $\widetilde{G}_{v_{m}}$ of the matrix $G_{\underline{v}_{m}}$. As in Section 3.1 above, in the contributions with $L=0,1,2,3$, the minor $\widetilde{G}_{\underline{v}_{m}}$ can be rewritten in a factorized form, analogue to (3.30), allowing to distinguish a contribution associated with the leaf $v^{*}$ from a rest associated with $\underline{v}_{m} \backslash v^{*}$.

The case $L=0$ can be treated as in item (A2.3). In fact, in this case the only coupling between the contribution associated with $v^{*}$ and the rest is through a single propagator belonging to $T_{v_{m}}$. It follows, by momentum conservation and 
the support properties of the single scale propagators (see the identity (2.135)), that the scale of $v_{m}$ is $\leq h+2$, so that the analysis in item (A2.3) can be repeated almost unchanged.

We now turn to the detailed analysis of the cases $L=1,2,3,4,5$.

(B.1a) $L=3$ : as in the example of Fig. 3, there are exactly three loop lines connecting $\tilde{P}_{v^{*}}$ with $\tilde{P}_{v_{m}} \backslash \tilde{P}_{v^{*}}$, call them $l_{1}, l_{2}, l_{3}$ and denote by $f_{1}, f_{2}, f_{3}, h_{1}, h_{2}, h_{3}$ and $\omega_{1}, \omega_{2}, \omega_{3}$ their field, scale and sector labels. With no loss of generality, we can assume $h \leq h_{1} \leq h_{2} \leq h_{3} \leq k$.

In this case we consider the propagators of $f_{1}, f_{2}, f_{3}, f^{*}$ and we expand them as sums of isotropic propagators, to be denoted by $\bar{g}_{\left.\bar{\omega}_{i}\right)}^{\left(h_{i}\right)}, i=1,2,3$ and $\bar{g}_{\bar{\omega}_{f^{*}}}^{(k)}$. If $h_{1}=h$, we rewrite the propagator $\bar{g}_{\bar{\omega}_{1}}^{h_{1}}\left(\mathbf{x}_{f_{1}^{+}}-\mathbf{y}_{f_{1}^{-}}\right)$in the form

$$
\bar{g}_{\bar{\omega}_{1}}^{h_{1}}\left(\mathbf{x}_{f_{1}^{+}}-\mathbf{y}_{f_{1}^{-}}\right)=\bar{g}_{\bar{\omega}_{1}}^{h_{1}}(\mathbf{0})+\left(\mathbf{x}_{f_{1}^{+}}-\mathbf{y}_{f_{1}^{-}}\right) \int_{0}^{1} d s \partial_{\mathbf{x}} \bar{g}_{\bar{\omega}_{1}}^{h_{1}}\left(s\left(\mathbf{x}_{f_{1}^{+}}-\mathbf{y}_{f_{1}^{-}}\right)\right) .
$$

If $h_{1}>h$, we expand the determinant of $\widetilde{G}_{\underline{v}_{m}}$ appearing in the analogue of $(3.15)$ along the row corresponding to a field label $f_{0}^{-} \in P_{v_{1}}^{-} \backslash P_{v_{0}}^{-}$(that is a field of scale $h$ ) and we rewrite

$$
\begin{gathered}
\operatorname{det} \widetilde{G}_{\underline{v}_{m}}=\sum_{f_{0}^{+} \in P_{v_{1}}^{+} \backslash P_{v_{0}}^{+}}(-1)^{\varepsilon_{0}} g_{\omega_{0}}^{(h)}\left(\mathbf{x}_{f_{0}^{+}}-\mathbf{y}_{f_{0}^{-}}\right) \cdot \\
\cdot \operatorname{det} \widetilde{G}_{\underline{v}_{m}}\left(\left\{f_{0}^{+}, f_{0}^{-}\right\}\right) \cdot \delta_{\omega\left(f_{0}^{+}\right), \omega\left(f_{0}^{-}\right)}, \\
g_{\omega_{0}}^{(h)}\left(\mathbf{x}_{f_{0}^{+}}-\mathbf{y}_{f_{0}^{-}}\right)=g_{\omega_{0}}^{(h)}(\mathbf{0})+\sum_{\bar{\omega}_{0} \prec \omega_{0}}\left(\mathbf{x}_{f_{0}^{+}}-\mathbf{y}_{f_{0}^{-}}\right) . \\
\cdot \int_{0}^{1} d s \partial_{\mathbf{x}} \bar{g}_{\bar{\omega}_{0}}^{(h)}\left(s\left(\mathbf{x}_{f_{0}^{+}}-\mathbf{y}_{f_{0}^{-}}\right)\right),
\end{gathered}
$$

where $\widetilde{G}_{\underline{v}_{m}}\left(\left\{f_{0}^{+}, f_{0}^{-}\right\}\right)$is the minor of $\widetilde{G}_{\underline{v}_{m}}$ corresponding to the entry $\left(f_{0}^{+}, f_{0}^{-}\right)$, $(-1)^{\varepsilon_{0}}$ is the corresponding sign and we denoted by $\omega_{0}$ the common value of $\omega\left(f_{0}^{ \pm}\right)$. See Fig. 11 for an example.

Both in the case $h_{1}=h$ and $h_{1}>h$, implementing the decompositions (3.50) or $(3.51)$, we see that the terms proportional to $\bar{g}_{\bar{\omega}_{1}}^{h_{1}}(\mathbf{0})$ or to $g_{\omega_{0}}^{(h)}(\mathbf{0})$ have a dimensional gain of $\gamma^{h}$, associated with the fact that the bound on $\bar{g}_{\bar{\omega}}^{(h)}(\mathbf{0})$ and on $g_{\omega}^{(h)}(\mathbf{0})$ are $\gamma^{h}$ smaller than the respective bounds on $\bar{g}_{\bar{\omega}}^{(h)}(\mathbf{x})$ and on $g_{\omega}^{(h)}(\mathbf{x})$, see Lemma 2.2, Lemma 2.3, Lemma 2.4 and Lemma 2.5.

So, in both cases, let us focus on the contributions proportional to the interpolated term in (3.50) and (3.51).

Such terms have essentially the same structure as the r.h.s. of (3.15) with $L=3$, unless for the following facts: the minor $\widetilde{G}^{h, T_{v_{0}}}$ is replaced by a suitable minor of the determinant $\operatorname{det} G_{\underline{v}_{m}}$ of all loop lines contracted on scales $h=h_{v_{0}}, h_{v_{1}}, \ldots, h_{v_{m}}=k$; if $h_{1}>h$ the number of extracted loop lines is 4 instead of 3 and their field labels are denoted by $f_{0}, f_{1}, f_{2}, f_{3}$; one of the loop lines 


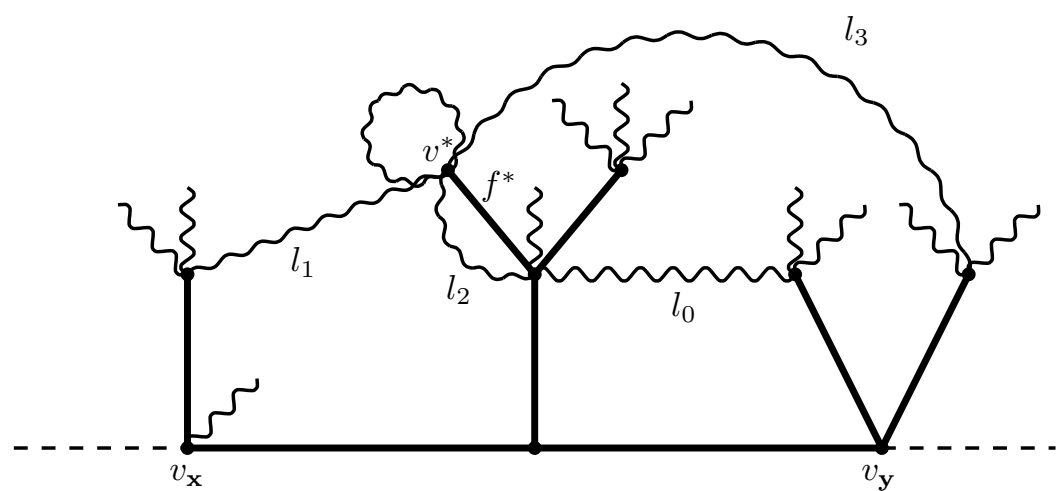

Figure 11. An example of case (B.1a).

( $f_{1}$ if $h_{1}=h$ or $f_{0}$ if $h_{1}>h$ ) is associated to an interpolated propagator (the second term in(3.50) or the second term in the second line of (3.51), respectively).

For any fixed configuration of anisotropic sectors, the new sums on the isotropic sectors, including possibly the sum over $\bar{\omega}_{0}$, can be written as:

$$
\prod_{i}^{*} \sum_{\bar{\omega}_{i} \prec \omega_{f_{i}}},
$$

where the $*$ on the product means that the product runs over $i=1,2,3$ if $h_{1}=h$ and over $i=0,1,2,3$ if $h_{1}>h$. Note also that, by construction, the summand in the expression under analysis (i.e., the analogue of the summand in the r.h.s. of $(3.15))$ is identically 0 unless $\chi\left(\left\{\bar{\omega}_{1}, \bar{\omega}_{2}, \bar{\omega}_{3}, \bar{\omega}_{f^{*}}\right\}\right)=1$. Then we are free to multiply the summand in the expression under analysis by $\chi\left(\left\{\bar{\omega}_{1}, \bar{\omega}_{2}, \bar{\omega}_{3}, \bar{\omega}_{f *}\right\}\right)$. However we choose to multiply it by a slightly weaker constraint, that is by $\chi\left(\left\{\bar{\omega}_{1}^{(k)}, \bar{\omega}_{2}^{(k)}, \bar{\omega}_{3}^{(k)}, \bar{\omega}_{f^{*}}\right\}\right)$, where $\bar{\omega}_{i}^{(k)}$ is the isotropic sector index on scale $k$ such that $\bar{\omega}_{i} \prec \bar{\omega}_{i}^{(k)}$ (note that by construction $\bar{\omega}_{f^{*}}$ is already on scale $k$ ).

Having done this, we can bound the resulting expression by replacing all the modified coupling functions, all the propagators and all the Gram determinants with their absolute values. As regarding the factor $\left(\mathbf{x}_{f_{1}^{+}}-\mathbf{y}_{f_{1}^{-}}\right)$or $\left(\mathbf{x}_{f_{0}^{+}}-\mathbf{y}_{f_{0}^{-}}\right)$ appearing in (3.50) or (3.51), we can bound its absolute value by a sum over the lines $l \in T^{\prime \prime}$ of $\left|\mathbf{x}_{l}-\mathbf{y}_{l}\right|$, where $T^{\prime \prime}$ is a suitable subset of the spanning tree $T^{\prime}$. In particular we can associate each factor $\left|\mathbf{x}_{l}-\mathbf{y}_{l}\right|$ to the corresponding propagator of the spanning tree $T^{\prime}$. Note that the number of terms in the sum $\sum_{l \in T^{\prime \prime}}$ is of order $n$.

Now, we can bound the integral over the absolute values of the modified coupling functions as in (2.102) (by using (3.18) in place of (2.92)), the integral over the absolute values of the propagators of the spanning tree times the factor $\sum_{l \in T^{\prime \prime}}\left|\mathbf{x}_{l}-\mathbf{y}_{l}\right|$ by the r.h.s. of (2.101) times a dimensional loss $\mathrm{cn}^{-k}$, where we used that the propagators associated to $l \in T^{\prime \prime}$ are of scale $j \geq k$ and that, 
by (2.61), $\int d \mathbf{x}|\mathbf{x}|\left|g_{\omega}^{(j)}(\mathbf{x})\right| \leq c \gamma^{-2 j}$. Moreover, the product of the determinants times the product of the absolute values of the extracted loop propagators can be bounded by the product over $v$ of the r.h.s. of (2.100) times a dimensional gain $\gamma^{h\left(1+\frac{1}{2} \mathbb{1}\left(h_{1}>h\right)\right)} \gamma^{\frac{1}{2}\left(h_{1}+h_{2}+h_{3}\right)}$ : the first factor $\gamma^{h}$ comes from the derivative $\partial_{\mathbf{x}}$ acting on $\bar{g}_{\bar{\omega}_{1}}^{(h)}$ or on $\bar{g}_{\bar{\omega}_{0}}^{(h)}$, see Lemma 2.4, while the remaining factors come from the improved bound on the size of the isotropic propagators with respect to the anisotropic ones.

After these bounds, the sums over the choices of the extracted loop propagators (including possibly the sum over $f_{0}^{+}$) can be bounded by $\left|P_{v_{1}}\right| \prod_{w \in \underline{v}_{m}}\left|P_{w}\right|^{3}$.

We are still left with the sum over the sector indices:

$$
\sum_{\Omega \backslash \Omega_{v_{0}}}\left[\prod_{i}^{*} \sum_{\bar{\omega}_{i} \prec \omega_{i}} \delta_{\omega_{f_{i}^{+}}, \omega_{f_{i}^{-}}}\right]\left[\prod_{\substack{v>v_{0} \\ v \in V_{c}}} \chi\left(\tilde{\Omega}_{v, \bar{\omega}}\right)\right] \chi\left(\left\{\bar{\omega}_{1}^{(k)}, \bar{\omega}_{2}^{(k)}, \bar{\omega}_{3}^{(k)}, \bar{\omega}_{f^{*}}\right\}\right)\left[\prod_{l \in T^{\prime}} \delta_{\omega_{l}^{+}, \omega_{l}^{-}}\right] \text {. }
$$

We now want to bound (3.53) and to compare the result we shall find with the bound we would get by proceeding as in Sect.2.8 above, that is with:

$$
c^{n} \prod_{\substack{v \in V_{c} \\ v \text { not e. p. }}} \gamma^{\frac{1}{2}\left(h_{v}-h_{v^{\prime}}\right)\left(\left|P_{v}\right|-3+211\left(\left|P_{v}\right| \geq 10\right)\right)} \prod_{v \text { e. p. }} \gamma^{-\frac{1}{2} h_{v}} .
$$

Using a notation completely analogous to the notations of 2.8, see (2.104) and following equations, we begin with bounding the product of $\chi$ functions in $(3.53)$ as

$$
\prod_{\substack{v>v_{0} \\ v \in V_{c}}} \chi\left(\tilde{\Omega}_{v, \bar{\omega}}\right) \leq \prod_{\substack{v>v_{m} \\ v \in V_{c}}} \chi\left(\tilde{\Omega}_{v, \bar{\omega}}^{(k)}\right)
$$

that is we simply neglect the constraints associated to the vertices $v_{1}, \ldots, v_{m}$ and we weaken the remaining constraints by replacing sectors on scales $\leq k$ with the corresponding sectors on scale $k$.

Also, we rewrite the sums over the sector indices in the form

$$
\begin{aligned}
\sum_{\Omega \backslash \Omega_{v_{0}}}\left[\prod_{i}^{*} \sum_{\bar{\omega}_{i} \prec \omega_{i}} \delta_{\omega_{f_{i}^{+}}, \omega_{f_{i}^{-}}}\right] & {\left[\prod_{i}^{*} \sum_{\bar{\omega}_{i}^{(k)} \in \bar{O}_{k}}\right] \cdot\left[\prod_{i}^{*} \sum_{\bar{\omega}_{i} \prec \bar{\omega}_{i}^{(k)}}\right] \cdot \sum_{\Omega_{v_{m}}^{(k)}}^{* *} \sum_{\Omega_{v_{m}} \prec \Omega_{v_{m}}^{(k)}}^{* *} \cdot \sum_{\Omega \backslash \Omega_{v_{m}}}^{* *} }
\end{aligned}
$$

where the $* *$ on the sums mean that we are not summing neither over the sectors in $\Omega_{v_{0}}$ nor over the sectors $\omega_{0}, \omega_{1}, \omega_{2}, \omega_{3}$. Let us denote by $P_{L}$ the set of field labels of the half-lines corresponding to the extracted loop propagators (equal to $\left\{f_{1}^{ \pm}, f_{2}^{ \pm}, f_{3}^{ \pm}\right\}$or to $\left\{f_{0}^{ \pm}, f_{1}^{ \pm}, f_{2}^{ \pm}, f_{3}^{ \pm}\right\}$, depending whether $h_{1}=h$ or not) and by $n_{i}^{L}=\left|P_{L} \cap P_{v_{i}}\right|$ the number of field labels in $P_{L}$ external to $v_{i}$. With this notation, we can bound 


$$
\begin{aligned}
{\left[\prod_{i}^{*} \sum_{\bar{\omega}_{i} \prec \bar{\omega}_{i}^{(k)}}\right] \leq c \gamma^{(k-h) \mathbb{1}\left(h_{1}>h\right)} \prod_{i=1}^{3} \gamma^{\left(k-h_{i}\right)} } \\
\sum_{\Omega_{v_{m}} \prec \Omega_{v_{m}}^{(k)}}^{* *} \leq c^{m} \prod_{i=1}^{m} \gamma^{\frac{1}{2}\left(h_{v_{i}}-h_{v_{i-1}}\right)\left(\left|P_{v_{i}}\right|-2-n_{i}^{L}\right)} \\
=c^{m} \gamma^{-(k-h) \mathbb{1}\left(h_{1}>h\right)} \prod_{i=1}^{3} \gamma^{-\left(k-h_{i}\right)} \prod_{i=1}^{m} \gamma^{\frac{1}{2}\left(h_{v_{i}}-h_{v_{i-1}}\right)\left(\left|P_{v_{i}}\right|-2\right)}
\end{aligned}
$$

Combining the two bounds in (3.57), we see that the global contribution from the sector sums corresponding to the vertices $v_{1}, \ldots v_{m}$ is proportional to $\prod_{i=1}^{m} \gamma^{\frac{1}{2}\left(h_{v_{i}}-h_{v_{i-1}}\right)\left(\left|P_{v_{i}}\right|-2\right)}$, to be compared with the corresponding bound $\prod_{i=1}^{m} \gamma^{\frac{1}{2}\left(h_{v_{i}}-h_{v_{i-1}}\right)\left(\left|P_{v_{i}}\right|-3+211\left(\left|P_{v}\right| \geq 10\right)\right)}$ obtained with the procedure of Section 2.8, see (3.54). We see that, as regards the sector sums corresponding to the vertices $v_{1}, \ldots v_{m}$, with respect to the corresponding bound in (3.54) the present bound has a dimensional loss at most equal to $\gamma^{\frac{1}{2}(k-h)}$.

After having bounded as above the sector sums corresponding to the vertices $v_{1}, \ldots v_{m}$, we are still left with the sector sums corresponding to the vertices $v$ $>v_{m}$, that can be rewritten in the form

$$
\begin{aligned}
{\left[\prod_{i}^{*} \sum_{\bar{\omega}_{i}^{(k)} \in \bar{O}_{k}}\right] } & \sum_{\cup_{v>v_{m}} \Omega_{v}^{(k)}}^{* *}\left[\prod_{v>v_{m}} \chi\left(\tilde{\Omega}_{v, \bar{\omega}}^{(k)}\right)\right] \chi\left(\left\{\bar{\omega}_{1}^{(k)}, \bar{\omega}_{2}^{(k)}, \bar{\omega}_{3}^{(k)}, \bar{\omega}_{f^{*}}\right\}\right) \\
\cdot & {\left[\prod_{l \in T^{\prime}} \delta_{\omega_{l}^{+}, \omega_{l}^{-}}\right]=\sum_{\Omega_{\underline{v}_{m}}^{(k)} \backslash \Omega_{v_{0}}}\left[\prod_{i}^{*} \sum_{\bar{\omega}_{i}^{(k)} \prec \omega_{i}^{(k)}} \delta_{\omega_{f_{i}}, \omega_{f_{i}^{-}}}\right] } \\
& \cdot \prod_{l \in T_{v_{m}}} \delta_{\omega_{l}^{+}, \omega_{l}^{-}}\left[\prod_{v \in \underline{v}_{m}} F_{v}\left(\tilde{\Omega}_{v, \bar{\omega}}^{(k)}\right)\right] \chi\left(\left\{\bar{\omega}_{1}^{(k)}, \bar{\omega}_{2}^{(k)}, \bar{\omega}_{3}^{(k)}, \bar{\omega}_{f^{*}}\right\}\right)
\end{aligned}
$$

Now, if $h_{1}=h$ (i.e., if the only extracted loop lines are $f_{1}, f_{2}, f_{3}$ ), defining $\Omega^{(4)}=$ $\left\{\omega_{1}, \omega_{2}, \omega_{3}, \omega_{f *}\right\}$, we see that we can soon bound (3.58) by an expression analogue to the r.h.s. of (3.21), so that, repeating the discussion after (3.21), we find that (3.58) can be bounded by the factor in (3.54) corresponding to the vertices $v>v_{m}$ times a dimensional loss of $\gamma^{-k / 2}|k|$. If $h_{1}>h$ (i.e., if the extracted loop lines are $\left.f_{0}, f_{1}, f_{2}, f_{3}\right)$ we bound the sum $\sum_{\bar{\omega}_{0}^{(k)}} \prec \omega_{0}^{(k)}$ by a constant times $\gamma^{-k / 2}$ and we neglect the constraint that the half-lines $f_{0}^{+}$and $f_{0}^{-}$have the same sector index (on scale $k$ ). After this, we are again left with an expression analogue to the r.h.s. of $(3.21)$, so that we lose a factor $\gamma^{-k / 2}|k|$ more.

In conclusion we can say that (3.58) can be bounded by a quantity that, with respect to the factor in (3.54) corresponding to the vertices $v>v_{m}$, has a loss of $\gamma^{-\frac{1}{2} k\left(1+11\left(h_{1}>h\right)\right)}|k|$. 
Combining all the gain and losses discussed in this item we find that the contributions under analysis, with respect to the bounds obtained via the procedure in Section 2.8, have a global gain of:

$$
\begin{aligned}
& c n \gamma^{-k} \gamma^{h} \gamma^{\frac{1}{2} h \mathbb{1}\left(h_{1}>h\right)} \gamma^{\frac{1}{2}\left(h_{1}+h_{2}+h_{3}\right)}\left|P_{v_{1}}\right|\left[\prod_{w \in \underline{v}_{m}}\left|P_{w}\right|^{3}\right] \\
& \cdot \gamma^{\frac{1}{2}(k-h)} \gamma^{-\frac{1}{2} k\left(1+\mathbb{1}\left(h_{1}>h\right)\right)}|k|=c n\left|P_{v_{1}}\right|\left[\prod_{w \in \underline{v}_{m}}\left|P_{w}\right|^{3}\right] \\
& \cdot \gamma^{h}|k| \gamma^{\frac{1}{2}(k-h)\left(1-11\left(h_{1}>h\right)\right)} \prod_{i=1}^{3} \gamma^{-\frac{1}{2}\left(k-h_{i}\right)} \leq c^{n} \gamma^{h}|h| \prod_{v \in V_{c}}\left|P_{v}\right|^{5},
\end{aligned}
$$

where in the last inequality we used the trivial bounds $c n \leq c^{n},|k| \leq|h|$ and $\left|P_{v_{1}}\right|$. $\cdot\left[\prod_{w \in \underline{v}_{m}}\left|P_{w}\right|^{3}\right] \leq \prod_{v \in V_{c}}\left|P_{v}\right|^{5}$.

(B.1b) $L=2$ : there are exactly two loop lines connecting $\tilde{P}_{v^{*}}$ with $\tilde{P}_{v_{m}} \backslash \tilde{P}_{v^{*}}$, see Fig. 12.

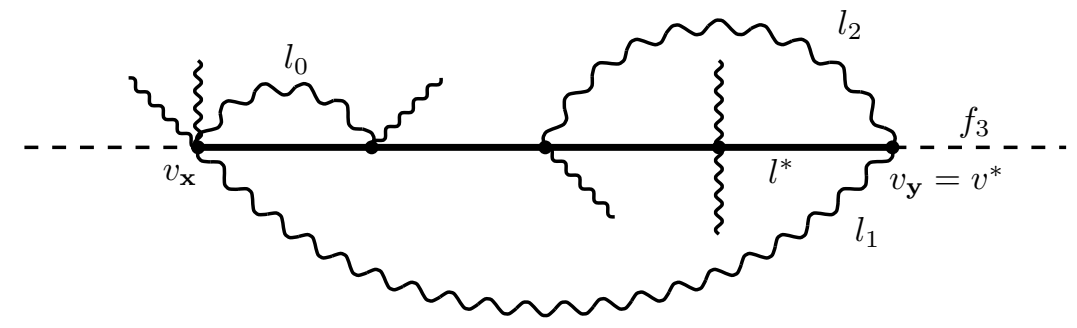

Figure 12. An example of case (B1.b).

We call $l_{1}, l_{2}$ the extracted loop lines, $f_{1}, f_{2}$ the corresponding field labels in $\tilde{P}_{v^{*}}, h_{1}, h_{2}$ their scale labels (assume $h \leq h_{1} \leq h_{2} \leq k$ ) and $\omega_{1}, \omega_{2}$ their sector labels. In this case we proceed in a way very similar to item (B.1a) above and we only rapidly repeat the proof of item (B.1a) adapted to the present case. The main difference consists in the fact that one of the fields in $P_{v^{*}}$, call it $f_{3}$, belongs to $P_{v_{0}}$, so that its sector label is fixed to be isotropic and equal to $\bar{\omega}$.

As in item (B.1a) we expand the propagators of $l_{1}, l_{2}$ and $l^{*}$ as sums of isotropic propagators and, depending whether $h_{1}=h$ or not, we also perform the expansions in (3.50) or (3.51); the example of Fig. 12 refers to the second case. Focusing on the contributions coming from the interpolated terms in (3.50), (3.51) (the others trivially admitting a gain of $\gamma^{h}$ ), we note that, for any fixed configuration of isotropic sectors, the new sums on isotropic sectors can be written as in (3.52), where the $*$ on the product now means that the product runs over $i=1,2$ if $h_{1}=h$ and over $i=0,1,2$ if $h_{1}>h$. We again choose to multiply the summand in the expression under analysis by $\chi\left(\left\{\bar{\omega}_{1}^{(k)}, \bar{\omega}_{2}^{(k)}, \bar{\omega}_{3}^{(k)}, \bar{\omega}_{f^{*}}\right\}\right)$, where now $\bar{\omega}_{3}^{(k)}$ must be interpreted as the isotropic sector index on scale $k$ such that $\bar{\omega} \prec \bar{\omega}_{3}^{(k)}$. 
Then we bound the product of coupling functions, the integrals over the propagators of the spanning tree (including the factor $\sum_{l \in T^{\prime \prime}}\left|\mathbf{x}_{l}-\mathbf{y}_{l}\right|$, see item (B.1a)) and the product of Gram determinants times the product of loop propagators as explained in item (B.1a) above. With respect to the naive bounds obtained by proceeding as in Sect.2.8, we lose a factor $n \gamma^{-k}$, because of the presence of $\sum_{l \in T^{\prime \prime}}\left|\mathbf{x}_{l}-\mathbf{y}_{l}\right|$, and we gain a factor $\gamma^{h\left(1+\frac{1}{2} \mathbb{1}\left(h_{1}>h\right)\right)} \gamma^{\frac{1}{2}\left(h_{1}+h_{2}\right)}$ because of the presence of the isotropic loop propagators and of the derivative acting on one isotropic loop propagator. Then, the sum over the choices of the extracted loop propagators gives a combinatorial factor $\left|P_{v_{1}}\right| \prod_{w \in \underline{v}_{m}}\left|P_{w}\right|^{2}$.

After this, we are left with the sum (3.53) over the sector indices, whose bound must be compared with the naive bound (3.54). We proceed again through (3.55) and (3.56) (where now the symbol $\prod_{i}^{*}$ must be interpreted as explained above), then we bound $\prod_{i}^{*} \sum_{\bar{\omega}_{i} \prec \bar{\omega}_{i}^{(k)}}$ and $\sum_{\Omega_{v_{m}} \prec \Omega_{v_{m}}^{(k)}}$ exactly as in $(3.57)$, unless for the fact that the product $\prod_{i=1}^{3}$ appearing in the r.h.s. of (3.57) must be replaced by $\prod_{i=1}^{2}$. So, exactly as in item (B.1a) above, the sector sums corresponding to the vertices $v_{1}, \ldots, v_{m}$ have a loss at most equal to $\gamma^{\frac{1}{2}(k-h)}$ with respect to the corresponding contribution in (3.54). We are still left with the sum in (3.58) and again, if $h_{1}=h$, we can reduce the analysis of the expression in (3.58) to the analysis of an expression similar to (3.41) of item (A2.1); then we find that such expression can be bounded by the factor in (3.54) corresponding to the vertices $v>v_{m}$ times a loss of $|k|$. If $h_{1}>h$ we bound the sum $\sum_{\bar{\omega}_{0}^{(k)} \prec \omega_{0}^{(k)}}$ by $c \gamma^{-k / 2}$ and we neglect the constraint that the half-lines contracted in $l_{0}$ have the same sector index (on scale $k$ ); then we are left again with an expression analogue to (3.41) and we find a loss of a factor $|k|$ more.

Combining all gains and losses we find that the global gain has an expression similar to (3.59):

$$
\begin{aligned}
& c n \gamma^{-k} \gamma^{h} \gamma^{\frac{1}{2} h \mathbb{1}\left(h_{1}>h\right)} \gamma^{\frac{1}{2}\left(h_{1}+h_{2}\right)}\left|P_{v_{1}}\right|\left[\prod_{w \in \underline{v}_{m}}\left|P_{w}\right|^{2}\right] \gamma^{\frac{1}{2}(k-h)} \\
& \cdot \gamma^{\left.-\frac{1}{2} k(1)\left(h_{1}>h\right)\right)}|k|=c n\left|P_{v_{1}}\right|\left[\prod_{w \in \underline{v}_{m}}\left|P_{w}\right|^{2}\right] \\
& \cdot \gamma^{h}|k| \gamma^{\left.\frac{1}{2}(k-h)(1-1)\left(h_{1}>h\right)\right)} \prod_{i=1}^{2} \gamma^{-\frac{1}{2}\left(k-h_{i}\right)} \leq c^{n} \gamma^{h}|h| \prod_{v \in V_{c}}\left|P_{v}\right|^{5} .
\end{aligned}
$$

(B.2a) $L=5$ : as in the example of Fig. 4, there are exactly five loop lines connecting $\tilde{P}_{v^{*}}$ with $\tilde{P}_{v_{m}} \backslash \tilde{P}_{v^{*}}$, call them $l_{1}, \ldots, l_{5}$ and denote by $f_{1}, \ldots, f_{5}$, by $h_{1}, \ldots, h_{5}$ and by $\omega_{1} \ldots, \omega_{5}$ their field, scale and sector labels. With no loss of generality, we can assume $h \leq h_{1} \leq \ldots h_{5} \leq k$. If $h_{1}=h$, then we rewrite the propagator $g_{\omega_{1}}^{h_{1}}\left(\mathbf{x}_{f_{1}^{+}}-\mathbf{y}_{f_{1}^{-}}\right)$in a form similar to $(3.50)$ :

$$
g_{\omega_{1}}^{h_{1}}\left(\mathbf{x}_{f_{1}^{+}}-\mathbf{y}_{f_{1}^{-}}\right)=g_{\omega_{1}}^{h_{1}}(\mathbf{0})+\sum_{\bar{\omega}_{1} \prec \omega_{1}}\left(\mathbf{x}_{f_{1}^{+}}-\mathbf{y}_{f_{1}^{-}}\right) \int_{0}^{1} d s \partial_{\mathbf{x}} \bar{g}_{\bar{\omega}_{1}}^{h_{1}}\left(s\left(\mathbf{x}_{f_{1}^{+}}-\mathbf{y}_{f_{1}^{-}}\right)\right)
$$


If $h_{1}>h$, then as in item (B.1a) above we expand the determinant of $\widetilde{G}_{\underline{v}_{m}}$ appearing in the analogue of (3.15) along the row corresponding to a field label $f_{0}^{-} \in P_{v_{1}}^{-} \backslash P_{v_{0}}^{-}$and we rewrite $\operatorname{det} \widetilde{G}_{\underline{v}_{m}}$ as in (3.51).

Both in the case $h_{1}=h$ and $h_{1}>h$, implementing the rewritings (3.61) or (3.51), we see that the terms proportional to $g_{\omega_{1}}^{h_{1}}(\mathbf{0})$ or to $g_{\omega_{0}}^{(h)}(\mathbf{0})$ have a dimensional gain of $\gamma^{h}$. So, in both cases, let us focus on the contributions proportional to the interpolated term in (3.60) and (3.51).

We replace all the modified coupling functions, all the propagators and all the Gram determinants with their absolute values. As before, we can bound the integral over the absolute values of the modified coupling functions as in (2.102) (by using (3.18) in place of (2.92)). The integral over the absolute values of the propagators of the spanning tree times the factor $\left(\mathbf{x}_{f_{1}^{+}}-\mathbf{y}_{f_{1}^{-}}\right)$or $\left(\mathbf{x}_{f_{0}^{+}}-\mathbf{y}_{f_{0}^{-}}\right)$is bounded again by the r.h.s. of (2.101) times a dimensional loss $c n \gamma^{-k}$. The product of the determinants times the product of the absolute values of the extracted loop propagators can be bounded by the product over $v$ of the r.h.s. of $(2.100)$ times a dimensional gain $\gamma^{\frac{3}{2}} h$, coming from the fact that the dimensional bound of the derived isotropic propagator on scale $h$ (the one corresponding to $f_{1}$ or to $f_{0}$, depending whether $h_{1}=h$ or not) is $\gamma^{\frac{3}{2} h}$ smaller than the corresponding bound for an anisotropic propagator on scale $h$. After these bounds, the sums over the choices of the loop propagators can be bounded by $\left|P_{v_{1}}\right| \prod_{w \in \underline{v}_{m}}\left|P_{w}\right|^{5}$.

We are still left with the sum over the sector indices:

$$
\sum_{\Omega \backslash \Omega_{v_{0}}} \sum_{\bar{\omega}_{i^{*}} \prec \omega_{i^{*}}}\left[\prod_{\substack{v>v_{0} \\ v \in V_{c}}} \chi\left(\tilde{\Omega}_{v, \bar{\omega}}\right)\right]\left[\prod_{l \in T^{\prime}} \delta_{\omega_{l}^{+}, \omega_{l}^{-}}\right]\left[\prod_{i}^{*} \delta_{\omega_{i}^{+}, \omega_{i}^{-}}\right],
$$

where the index $i^{*}$ attached to the sector indices in the second sum is $i^{*}=1,0$, depending whether $h_{1}=h$ or not, and the $*$ on the last product means that the product ranges over $i=1,2,3,4,5$ or over $i=0,1,2,3,4,5$, depending whether $h_{1}=h$ or not.

We now want to bound (3.62) and to compare the result we shall find with (3.54). We again bound the product of $\chi$ functions as in (3.55). We bound the sum $\sum_{\bar{\omega}_{i *} \prec \omega_{i^{*}}}$ by a constant times $\gamma^{-h / 2}$ and, after this, we rewrite

$$
\sum_{\Omega \backslash \Omega_{v_{0}}}\left[\prod_{i}^{*} \delta_{\omega_{i}^{+}, \omega_{i}^{-}}\right]=\left[\prod_{i}^{*} \sum_{\omega_{i}^{(k)} \in O_{k}}\right] \cdot\left[\prod_{i}^{*} \sum_{\omega_{i} \prec \omega_{i}^{(k)}}\right] \cdot \sum_{\Omega_{v_{m}}^{(k)}}^{* *} \cdot \sum_{\Omega_{v_{m}} \prec \Omega_{v_{m}}^{(k)}}^{* *} \cdot \sum_{\Omega \backslash \Omega_{v_{m}}}^{* *}
$$

where the $* *$ on the sums mean that we are not summing neither over the sectors in $\Omega_{v_{0}}$ nor over the sectors $\omega_{0}, \omega_{1}, \ldots, \omega_{5}$. If we denote by $P_{L}$ the set of field labels of the half-lines corresponding to the extracted loop propagators (equal to $\left\{f_{1}^{ \pm}, \ldots, f_{5}^{ \pm},\right\}$or to $\left\{f_{0}^{ \pm}, f_{1}^{ \pm}, \ldots, f_{5}^{ \pm}\right\}$, depending whether $h_{1}=h$ or not) and by $n_{i}^{L}=\left|P_{L} \cap P_{v_{i}}\right|$ the number of field labels in $P_{L}$ external to the cluster $v_{i}$, we can bound 


$$
\begin{aligned}
{\left[\prod_{i}^{*} \sum_{\omega_{i} \prec \omega_{i}^{(k)}}\right] \leq c \gamma^{\frac{1}{2}(k-h) \mathbb{1}\left(h_{1}>h\right)} \prod_{i=1}^{5} \gamma^{\frac{1}{2}\left(k-h_{i}\right)} } \\
\sum_{\Omega_{v_{m}} \prec \Omega_{v_{m}}^{(k)}}^{* *} \leq c^{m} \prod_{i=1}^{m} \gamma^{\frac{1}{2}\left(h_{v_{i}}-h_{v_{i-1}}\right)\left(\left|P_{v_{i}}\right|-2-n_{i}^{L}\right)} \\
=c^{m} \gamma^{-(k-h) \mathbb{1}\left(h_{1}>h\right)} \prod_{i=1}^{5} \gamma^{-\left(k-h_{i}\right)} \prod_{i=1}^{m} \gamma^{\frac{1}{2}\left(h_{v_{i}}-h_{v_{i-1}}\right)\left(\left|P_{v_{i}}\right|-2\right)}
\end{aligned}
$$

Combining the two bounds in (3.64), we see that the global contribution from the sector sums corresponding to the vertices $v_{1}, \ldots v_{m}$ is proportional to $\prod_{i=1}^{m} \gamma^{\frac{1}{2}\left(h_{v_{i}}-h_{v_{i-1}}\right)\left(\left|P_{v_{i}}\right|-2\right)} \gamma^{-\frac{1}{2}(k-h) 1\left(h_{1}>h\right)} \prod_{i=1}^{5} \gamma^{-\frac{1}{2}\left(k-h_{i}\right)}$. Comparing this with the corresponding bound in (3.54), we see that the present bound has a dimensional gain at least equal to $\gamma^{-\frac{1}{2}(k-h)\left(\mathbb{1}\left(h_{1}>h\right)-1\right)} \prod_{i=1}^{5} \gamma^{-\frac{1}{2}\left(k-h_{i}\right)}$.

After having bounded as above the sector sums corresponding to the vertices $v_{1}, \ldots v_{m}$, we are still left with the sector sums corresponding to the vertices $v$ $>v_{m}$, that can be rewritten in the form

$$
\begin{aligned}
{\left[\prod_{i}^{*} \sum_{\omega_{i}^{(k)} \in O_{k}}\right] \sum_{\cup_{v>v_{m}} \Omega_{v}^{(k)}}^{* *}\left[\prod_{v>v_{m}} \chi\left(\tilde{\Omega}_{v, \bar{\omega}}^{(k)}\right)\right]\left[\prod_{l \in T^{\prime}} \delta_{\omega_{l}^{+}, \omega_{l}^{-}}\right] } \\
=\sum_{\Omega_{\underline{v}_{m} \backslash \Omega_{v_{0}}}^{(k)}} \prod_{i}^{*} \delta_{\omega_{i}^{+}, \omega_{i}^{-}} \prod_{l \in T_{v_{m}}} \delta_{\omega_{l}^{+}, \omega_{l}^{-}}\left[\prod_{v \in \underline{v}_{m}} F_{v}\left(\tilde{\Omega}_{v, \bar{\omega}}^{(k)}\right)\right] .
\end{aligned}
$$

If we now discard the constraint coming from $\delta_{\omega_{f_{0}}^{+}, \omega_{f_{0}}^{-}}$(whenever present in (3.65)), we are left with an expression completely analogous to (3.27) so that, proceeding as in item (A1.2), we find that (3.65) admits a bound that, with respect to the corresponding bound in (3.54), has a dimensional gain of $\gamma^{k}$.

Combining all the gain and losses discussed in this item we find that the contributions under analysis, with respect to the bounds obtained via the procedure in Section 2.8, have a global gain of

$$
\begin{gathered}
c n \gamma^{-k} \gamma^{\frac{3}{2} h}\left|P_{v_{1}}\right|\left[\prod_{w \in \underline{v}_{m}}\left|P_{w}\right|^{5}\right] \gamma^{-\frac{1}{2} h} \gamma^{-\frac{1}{2}(k-h)\left(11\left(h_{1}>h\right)-1\right)} \\
\cdot\left[\prod_{i=1}^{5} \gamma^{-\frac{1}{2}\left(k-h_{i}\right)}\right] \gamma^{k}=c n\left|P_{v_{1}}\right|\left[\prod_{w \in \underline{v}_{m}}\left|P_{w}\right|^{5}\right] \\
\cdot \gamma^{h} \gamma^{-\frac{1}{2}(k-h)\left(11\left(h_{1}>h\right)-1\right)}\left[\prod_{i=1}^{5} \gamma^{-\frac{1}{2}\left(k-h_{i}\right)}\right] \leq c^{n} \gamma^{h} \prod_{v \in V_{c}}\left|P_{v}\right|^{5}
\end{gathered}
$$

(B.2b) $L=4$ : there are exactly four loop lines connecting $\tilde{P}_{v^{*}}$ with $\tilde{P}_{v_{m}} \backslash \tilde{P}_{v^{*}}$. We call $f_{1}, f_{2}, f_{3}, f_{4}$ the field labels of the extracted loop lines, $h_{1}, h_{2}, h_{3}, h_{4}$ their scale 
labels (assume $h \leq h_{1} \leq \cdots \leq h_{4} \leq k$ ) and $\omega_{1}, \omega_{2}, \omega_{3}, \omega_{4}$ their sector labels. In this case we proceed in a way very similar to item (B.2a) above and we only rapidly repeat the proof of item (B.2a) adapted to the present case. The main difference consists in the fact that one of the fields in $P_{v^{*}}$, call it $f_{5}$, belongs to $P_{v_{0}}$, so that its sector label is fixed to be isotropic and equal to $\bar{\omega}$.

As in item (B.2a), depending whether $h_{1}=h$ or not, we perform the expansions in (3.61) or in (3.51). We again focus on the contributions coming from the interpolated terms in $(3.61),(3.51)$ (the others trivially admitting a gain of $\gamma^{h}$ ).

We bound the product of coupling functions, the integrals over the propagators of the spanning tee (including the factor $\sum_{l \in T^{\prime \prime}}\left|\mathbf{x}_{l}-\mathbf{y}_{l}\right|$, see item (B.1a)) and the product of Gram determinants times the product of loop propagators as explained in item (B.2a) above. With respect to the naive bounds obtained by proceeding as in Sect.2.8, we lose a factor $n \gamma^{-k}$, because of the presence of $\sum_{l \in T^{\prime \prime}}\left|\mathbf{x}_{l}-\mathbf{y}_{l}\right|$, and we gain a factor $\gamma^{\frac{3}{2} h}$ because of the presence of one derived isotropic loop propagators. The sum over the choices of the extracted loop propagators gives a combinatorial factor $\left|P_{v_{1}}\right| \prod_{w \in \underline{v}_{m}}\left|P_{w}\right|^{4}$.

After this we are left with the sum (3.62) over the sector indices (where now the $*$ on the last product means that the product ranges over $i=1,2,3,4$ or over $i=0,1,2,3,4$, depending whether $h_{1}=h$ or not), whose bound must be compared with the naive bound (3.54).

We proceed again through $(3.55)$, we bound $\sum_{\bar{\omega}_{i^{*}} \prec \omega_{i^{*}}}$ by a constant times $\gamma^{-h / 2}$ and we consider the rewriting (3.63) (where now the symbol $\prod_{i}^{*}$ must be interpreted as explained above). We bound $\prod_{i}^{*} \sum_{\omega_{i} \prec \omega_{i}^{(k)}}$ and $\sum_{\Omega_{v_{m}} \prec \Omega_{v_{m}}^{(k)}}^{* *}$ exactly as in (3.64), unless for the fact that the product $\prod_{i=1}^{5}$ appearing in the r.h.s. of (3.64) must be replaced by $\prod_{i=1}^{4}$. So, exactly as in item (B.2a) above, the sector sums corresponding to the vertices $v_{1}, \ldots, v_{m}$ have a gain at least equal to $\gamma^{-\frac{1}{2}(k-h)\left(1\left(h_{1}>h\right)-1\right)} \prod_{i=1}^{4} \gamma^{-\frac{1}{2}\left(k-h_{i}\right)}$ with respect to the corresponding contribution in (3.54). We are still left with the sum in (3.65) and again we discard the constraint coming from $\delta_{\omega_{0}^{+}, \omega_{0}^{-}}$(whenever present in $(3.65)$ ); in this way we are reduced to an expression completely analogous to the one considered in item (A2.2), see (3.45), and we find that such expression can be bounded by the factor in (3.54) corresponding to the vertices $v>v_{m}$ times a gain of $\gamma^{k}$.

Combining all gains and losses we find that the global gain has an expression similar to $(3.66)$ :

$$
\begin{gathered}
c n \gamma^{-k} \gamma^{\frac{3}{2} h}\left|P_{v_{1}}\right|\left[\prod_{w \in \underline{v}_{m}}\left|P_{w}\right|^{4}\right] \gamma^{-\frac{1}{2} h} \gamma^{-\frac{1}{2}(k-h)\left(11\left(h_{1}>h\right)-1\right)} \\
\cdot\left[\prod_{i=1}^{4} \gamma^{-\frac{1}{2}\left(k-h_{i}\right)}\right] \gamma^{k}=c n\left|P_{v_{1}}\right|\left[\prod_{w \in \underline{v}_{m}}\left|P_{w}\right|^{4}\right] \\
\cdot \gamma^{h} \gamma^{-\frac{1}{2}(k-h)\left(11\left(h_{1}>h\right)-1\right)}\left[\prod_{i=1}^{4} \gamma^{-\frac{1}{2}\left(k-h_{i}\right)}\right] \leq c^{n} \gamma^{h} \prod_{v \in V_{c}}\left|P_{v}\right|^{5} .
\end{gathered}
$$


(B.3) $L=1$; as in the example of Fig. 5 , there is exactly one loop line connecting $\tilde{P}_{v^{*}}$ with $\tilde{P}_{v_{m}} \backslash \tilde{P}_{v^{*}}$. We call $l_{1}$ the extracted loop line and $f_{1}, h_{1}, \omega_{1}$ its field, scale and sector labels. Let us rewrite the propagators of $l^{*}$ and $l_{1}$ as sums of isotropic propagators, to be denoted by $\bar{g}_{\bar{\omega}_{f^{*}}}^{(k)}(\mathbf{x})$ and $g_{\bar{\omega}_{1}}^{\left(h_{1}\right)}(\mathbf{x})$. Note that, by momentum conservation and the support properties of the single scale propagators (see the identity $(2.135)), h_{1} \geq k-2$, so that it is sufficient to consider the case $h_{1}=k$; the general case will differ only in irrelevant details. Moreover, the momentum conservation also implies that the summand in the expression under analysis can be freely multiplied by $\mathbb{1}\left(\left|\bar{\omega}_{f^{*}}-\bar{\omega}_{1}\right| \leq 1\right)$.

Note also that the determinant $\operatorname{det} \widetilde{G}_{\underline{v}_{m}}$ of the loop lines contracted on scales $h=h_{v_{0}}, h_{v_{1}}, \ldots, h_{v_{m}}=k$, except those contracted in $l_{1}$, can be factorized in a form analogous to $(3.30)$, to be denoted by $\operatorname{det} \widetilde{G}_{v^{*}} \cdot \operatorname{det} \widetilde{G}_{\underline{v}_{m} \backslash v^{*}}$. Let us call $j_{*} \in[h, k]$ the smallest scale among those of the propagators contracted into $\operatorname{det} \widetilde{G}_{v^{*}}$ and $\bar{j} \in[h, k]$ the smallest scale among those of the propagators contracted into $\operatorname{det} \widetilde{G}_{\underline{v}_{m} \backslash v^{*}}$; the indices of the vertices with scale $j_{*}$ and $\bar{j}$ will be called $i_{*}$ and $\bar{i}$. Note that at least one among $j_{*}$ and $\bar{j}$ is equal to $h$.

For any choice of $l_{1}$ and any vertex $v_{i}, i=0, \ldots, m$, we can split the set $P_{v_{i}}$ as $P_{v_{i}}=P_{v_{i}}^{\prime} \cup P_{v_{i}}^{\prime \prime} \cup P_{v_{0}}$, where $P_{v_{i}}^{\prime}$ and $P_{v_{i}}^{\prime \prime}$ are the sets of external lines of $v_{i}$ contracted in $\operatorname{det} \widetilde{G}_{v^{*}}$ and $\operatorname{det} \widetilde{G}_{\underline{v}_{m} \backslash v^{*}}$, respectively. Then we define two trees $\tau^{*}$ and $\bar{\tau}$, by adding to the two subtrees of $\tau$ with first vertex $v_{m}$, the first one containing $v^{*}$ and the other containing $\left[\underline{v}_{m} \backslash v^{*}\right]$, the vertices $v_{i_{*}}, \ldots, v_{m-1}$ and $v_{\bar{i}}, \ldots, v_{m-1}$, respectively; see Fig. 13 for an example.

If $v \in \tau^{*}$ and $v=v_{i}, i=i^{*}, \ldots, m$, we shall put $P_{v}^{*}=P_{v_{i}}^{\prime} \cup\left\{f_{1}, f^{*}\right\}$; if $v \in \bar{\tau}$ and $v=v_{i}, i=\bar{i}, \ldots, m$, we shall put $\bar{P}_{v}=P_{v_{i}}^{\prime \prime} \cup P_{v_{0}} \cup\left\{\bar{f}_{1}, \bar{f}^{*}\right\}, \bar{f}_{1}$ and $\bar{f}^{*}$ being the lines contracted in $l_{1}$ and $l^{*}$ and not belonging to $P_{v^{*}}$. If $v>v_{m}$, we shall put $P_{v}^{*}=P_{v}$ if $v \in \tau^{*}, \bar{P}_{v}=P_{v}$ if $v \in \bar{\tau}$. Moreover, we shall call $T^{*}$ the subset

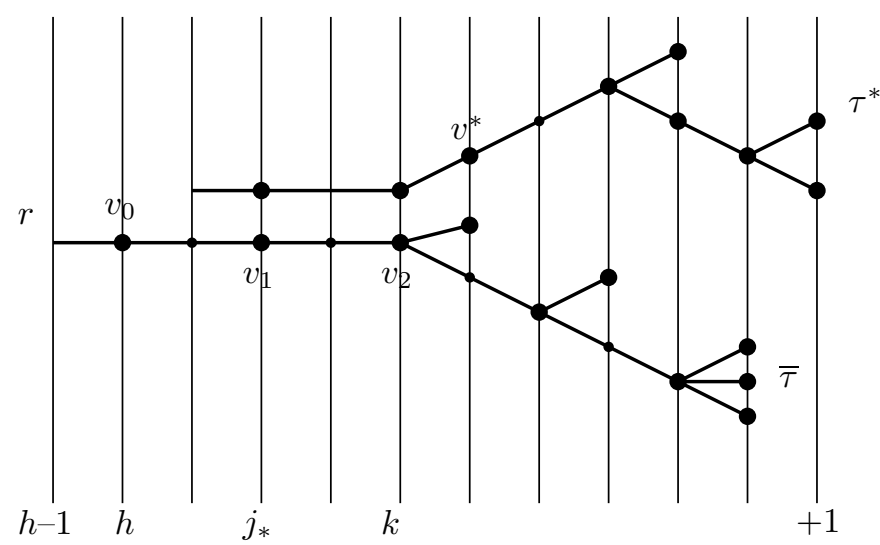

Figure 13. An example of $\tau^{*}$ and $\bar{\tau}$. 
of the spanning tree $T$ associated with all the vertices $v \geq v^{*}$, while $\bar{T}$ will be $T \backslash\left\{T^{*}, l^{*}\right\}$.

Note now that, for any $i=0, \ldots, m$, the constraint $\chi\left(\tilde{\Omega}_{v_{i}, \bar{\omega}}\right)$ is indeed independent of the two external lines in $P_{v_{0}}$, since they have the same momentum and belong both to $P_{v_{i}}$. It follows, by taking also in account that there is a separate conservation of momenta constraint for the sets $P_{v_{i}}^{\prime}$ and $P_{v_{i}}^{\prime \prime}$, that $\chi\left(\tilde{\Omega}_{v_{i}, \bar{\omega}}\right)=\chi\left(\Omega\left(P_{v_{i}}^{\prime}\right)\right) \chi\left(\Omega\left(P_{v_{i}}^{\prime \prime}\right)\right)$. Hence it is easy to see that we can bound the expression under analysis by the following one:

$$
\begin{array}{r}
\sum_{l_{1}} \sum_{\bar{\omega}_{1}, \bar{\omega}_{f}} \mathbb{1}\left(\left|\bar{\omega}_{f^{*}}-\bar{\omega}_{1}^{(k)}\right| \leq 1\right)\left[v^{*}\right]\left[\underline{v}_{m} \backslash v^{*}\right] \sup _{\mathbf{x}}\left|\bar{g}_{\bar{\omega}_{1}}^{(k)}(\mathbf{x})\right| \int d \mathbf{x}\left|\bar{g}_{\bar{\omega}_{f}^{*}}^{(k)}(\mathbf{x})\right| \\
\leq c\left|P_{v^{*}}\right|\left(\sum_{v \in \underline{v}_{m} \backslash v^{*}}\left|P_{v}\right|\right)\left[v^{*}\right]\left[\underline{v}_{m} \backslash v^{*}\right],
\end{array}
$$

where $\left[v^{*}\right]$ and $\left[\underline{v}_{m} \backslash v^{*}\right]$ have a structure very similar to $J_{j_{*}, m_{4}\left(v^{*}\right)}^{(2)}\left(\tau^{*}, \mathbf{P}^{*}, T^{*}\right)$ with $\left|P_{v_{i^{*}}}^{*}\right|=2$ and $J_{\bar{j}, n-m_{4}\left(v^{*}\right)}^{(4)}(\bar{\tau}, \overline{\mathbf{P}}, \bar{T})$ with $\left|\bar{P}_{v_{\bar{i}}}\right|=4$, with the following main differences.

1) The two external legs of $v_{i^{*}}$ and two of the four external legs of $v_{\bar{i}}$ have scale $k$ instead of $j^{*}$ or $\bar{j}$.

2) If $v \in \tau^{*}$ and $v=v_{i}, i=i^{*}+1, \ldots, m$, then the minimum value of $\left|P_{v}^{*}\right|$ can be equal to 4 , instead of 6 .

3) If $v \leq v_{m}$, the external legs involved in the conservation of momentum constraint are those belonging to $P_{v_{i}}^{\prime}$ (whose number is $\left|P_{v}^{*}\right|-2$ ), if $v \in \tau^{*}$, or those belonging to $P_{v_{i}}^{\prime \prime}$ (whose number is $\left|\bar{P}_{v}\right|-4$ ), if $v \in \bar{\tau}$.

Let us consider first $\left[\underline{v}_{m} \backslash v^{*}\right]$. We begin with bounding the sum over the sector indices. By extending in an obvious way the previous definitions, we can write

$$
\prod_{\substack{v>v_{\bar{i}} \\ v \in V_{c}}} \chi\left(\tilde{\Omega}_{v, \bar{\omega}, \bar{\omega}_{1}, \bar{\omega}_{f^{*}}}\right)=\prod_{i=\bar{i}+1}^{m} \chi\left(\Omega\left(P^{\prime \prime}\left(v_{i}\right)\right) \prod_{\substack{v>v_{m} \\ v \in V_{c}}} \chi\left(\tilde{\Omega}_{v, \bar{\omega}, \bar{\omega}_{1}, \bar{\omega}_{f *}}\right),\right.
$$

so that, using the item 3) above (together with momentum conservation, which allows us to eliminate one sector sum in each vertex) and calling $\bar{\Omega}_{\text {ext }}$ the sector indices of the legs in $P_{v_{\bar{r}}}$, we get

$$
\begin{aligned}
\sum_{\bar{\Omega} \backslash \bar{\Omega}_{\mathrm{ext}}} \prod_{\substack{v>v_{\bar{i}} \\
v \in V_{c}}} \chi\left(\tilde{\Omega}_{\left.v, \bar{\omega}, \bar{\omega}_{1}, \bar{\omega}_{f^{*}}\right)}\right. & \\
& \leq \prod_{i=\bar{i}+1}^{m} \gamma^{\frac{1}{2}\left(h_{v_{i}}-h_{v_{i-1}}\right)\left(\left|\bar{P}_{v_{i}}\right|-5\right)} \sum_{\bar{\Omega}^{(k)} \backslash \bar{\Omega}_{\mathrm{ext}}^{(k)}} \prod_{\substack{v>v_{m} \\
v \in V_{c}}} \chi\left(\tilde{\Omega}_{v, \bar{\omega}, \bar{\omega}_{1}, \bar{\omega}_{f^{*}}}^{(k)}\right) .
\end{aligned}
$$


Let us now consider $\operatorname{det} \widetilde{G}_{\underline{v}_{m} \backslash v^{*}}$; we can bound it as

$$
\begin{aligned}
\left|\operatorname{det} \widetilde{G}_{\underline{v}_{m} \backslash v^{*}}\right| & \leq\left[\prod_{i=\bar{i}}^{m-1}\left(c \gamma^{\frac{3}{4} h_{v_{i}}}\right)^{\left|\bar{P}_{v_{i+1}}\right|-\left|\bar{P}_{v_{i}}\right|}\right]\left(c \gamma^{\frac{3}{4} h_{v_{m}}}\right)^{L_{m}} \\
= & {\left[\prod_{i=\bar{i}}^{m-1} \gamma^{\frac{3}{4}\left(h_{v_{i}}-h_{v_{m}}\right)\left(\left|\bar{P}_{v_{i+1}}\right|-\left|\bar{P}_{v_{i}}\right|\right)}\right]\left[c^{\mid \bar{P}_{v_{m} \mid}+L_{m}-4} \gamma^{\frac{3}{4} h_{v_{m}} L_{\leq m}}\right], }
\end{aligned}
$$

where $L_{m}$ and $L_{\leq m}$ denote the number of loop lines contracted on scale $h_{m}$ and less or equal to $h_{m}$, respectively.

Let us now observe that, if we extract from the bound of $\left[\underline{v}_{m} \backslash v^{*}\right]$ the first terms in the r.h.s. of (3.70) and (3.71), we are left with an expression, which has the same structure of a contribution to the effective potential on scale $k$ with four external legs of fixed sector of the same scale. By using Lemma 2.7, this quantity can then be bounded by $(c|U|)^{n-m_{4}\left(v^{*}\right)} \prod_{v>v_{m}}\left(s_{v} !\right)^{-1} \gamma^{\delta\left(\left|P_{v}\right|\right)}$. On the other hand, the product of the first terms in the r.h.s. of (3.70) and (3.71) is equal to $\prod_{v_{\bar{\imath}}<v \leq v_{m}} \gamma^{-(1 / 4)\left(\left|\bar{P}_{v}\right|-2\right)}$. It follows that

$$
\left[\underline{v}_{m} \backslash v^{*}\right] \leq(c|U|)^{n-m_{4}\left(v^{*}\right)} \prod_{v_{\bar{i}}<v \leq v_{m}} \gamma^{-\frac{1}{4}\left(\left|\bar{P}_{v}\right|-2\right)} \prod_{v>v_{m}} \frac{1}{s_{v} !} \gamma^{\delta\left(\left|P_{v}\right|\right)} .
$$

Let us now consider $\left[v^{*}\right]$. If $v^{*}$ is an endpoint, by using (3.8), we see that $\left[v^{*}\right] \leq c|U||k| \gamma^{2 j_{*}}$. Moreover, since $\left|P_{v}\right| \geq 6$ for any $v>v_{0}, \bar{i}=0$ independently of the value of $j_{*}$ and $\left|\bar{P}_{v}\right|-2=\left|P_{v}\right|$ if $v_{0} \leq v \leq v_{i^{*}}$, while $\left|\bar{P}_{v}\right|=\left|P_{v}\right|$ if $v_{i^{*}}<v \leq v_{m}$. It follows that the r.h.s. of (3.68) can be bounded by

$$
(c|U|)^{n}\left(\prod_{v \in \underline{v}_{m}}\left|P_{v}\right|\right)|k| \gamma^{2 j_{*}} \gamma^{-\left(j_{*}-h\right)} \prod_{v>v_{0}} \frac{1}{s_{v} !} \gamma^{\delta\left(\left|P_{v}\right|\right)},
$$

which can be bounded by the r.h.s. of (3.6) (our goal) times a "bad factor" $\gamma^{j_{*}-h}$. However we can still improve the previous estimate, by extracting one propagator of scale $h$ from the determinant of the loop lines belonging to $\bar{P}_{v_{1}}$, as in item (B.1a), using (3.50). In this way, the bound (3.73) is multiplied by a factor $c\left|P_{v_{1}}\right|^{2}\left(n-m_{4}\left(v^{*}\right)\right) \gamma^{-(k-h)}$ and we are done.

We still have to consider the possibility that $v^{*}$ is not an endpoint. If $j_{*}=k$ the problem we have to face is exactly equal to the problem we are studying, so that we can suppose inductively that the bound (3.6) is satisfied. The induction is on the number of endpoints and stops when the vertex which takes the role of $v^{*}$ at each step of the inductive procedure is an endpoint. Hence, we get again the bound (3.73) and we can improve it by a factor $c\left|P_{v_{1}}\right|^{2}\left(n-m_{4}\left(v^{*}\right)\right) \gamma^{-(k-h)}$, since $\bar{i}=0$.

If $j_{*}<k$, the structure of $\left[v^{*}\right]$ is not exactly equal to that of the 1.h.s. in (3.6), because of the remarks 1)-3) after (3.68), but it is enough similar to apply again the procedure described in item (B). To be more precise, we consider the 
highest vertex $v \geq v^{*}$, such that $s_{v}>1$, let us call it $v_{0}^{*}$, and we expand as before the determinant of all the loop lines of scale between $h_{v_{0}^{*}}$ and $j_{*}$. The terms of the expansion with $L>1$ can be treated as before, since the procedure described in items (B.1a), (B.1b), (B.2a), (B.2b) does use the momentum constraints on the scales larger than $h_{v_{0}^{*}}$, see (3.55). It follows that

$$
\left[v^{*}\right] \leq(c|U|)^{m_{4}\left(v^{*}\right)}|k| \gamma^{2 j_{*}} \prod_{v_{i}<v \leq v^{*}} \gamma^{1-\left|P_{v}^{*}\right| / 4} \prod_{v>v^{*}} \frac{1}{s_{v} !}\left|P_{v}\right|^{5} \gamma^{\delta\left(\left|P_{v}\right|\right)}
$$

By using (3.72) and (3.74), together with the remarks that at least one among $\bar{i}$ and $i_{*}$ is equal to $h$ and that $\left|\bar{P}_{v}\right|+\left|P_{v}^{*}\right|-2=\left|P_{v}\right|$, if $\max \left\{v_{\bar{i}}, v_{i^{*}}\right\}<v \leq v_{m}$, we see that the r.h.s. of (3.68) can be bounded by

$$
(c|U|)^{n}|k| \gamma^{j_{*}+h}\left(\prod_{v>v_{m}}\left|P_{v}\right|^{5}\right) \prod_{v>v_{0}} \frac{1}{s_{v} !} \gamma^{\delta\left(\left|P_{v}\right|\right)},
$$

and, if $j_{*}>h$, once again we can improve the bound by a factor $c\left|P_{v_{1}}\right|^{2}(n-$ $\left.m_{4}\left(v^{*}\right)\right) \gamma^{-(k-h)}$, since $\bar{i}=0$. The final expression will be bounded by the r.h.s. of (3.6).

It remain to consider the terms in the determinant expansion with $L=0,1$. It easy to see that, in both cases, we have to extract the dimensional gain from a term with the same structure of $\left[v^{*}\right]$, but a smaller number of endpoints. So we can proceed by induction, which ends as soon as the analogue of $v^{*}$ is an endpoint; we omit the details, which would be obvious at this point. We only stress that in this iteration each vertex acquires, with respect to the bound (2.97), at most a factor $\left|P_{v}\right|^{5}$ and that each propagator of the spanning tree $T$ is multiplied at most one time for factor $\left|\mathbf{x}_{l}-\mathbf{y}_{l}\right|$, as a consequence of the "tadpole gain" operation (3.50), so that there is no risk of factorials in the bound.

This concludes the proof of (3.6) with $j=0$ under the assumptions (2.92), (3.18) and (3.8). The validity of (3.6) with $j \geq 1$ under the same assumptions is a trivial generalization of the proof above. In fact, in order to take into account the factor $|\mathbf{x}-\mathbf{y}|^{j}$ in the r.h.s. of (3.5), we can bound it by $(c n)^{j-1} \sum_{l \in T^{\prime \prime}}\left|\mathbf{x}_{l}-\mathbf{y}_{l}\right|^{j}$, where $T^{\prime \prime} \subset T$ is a path on $T$ such that $(\mathbf{x}-\mathbf{y})=\sum_{l \in T^{\prime \prime}}\left(\mathbf{x}_{l}-\mathbf{y}_{l}\right)$. Each term $\left|\mathbf{x}_{l}-\mathbf{y}_{l}\right|^{j}$ in this sum can be associated to the corresponding propagator of $l \in T$ : then, when performing the integrations over the propagators of the spanning tree, each of these terms contributes with a dimensional factor $\leq \gamma^{-h j}$, see Remarks following Lemma 2.2 and Lemma 2.4. Then, for any integer $j \geq 0,(3.6)$ under the assumptions (2.92), (3.18) and (3.8) follows.

\subsection{Proof of the bounds (2.92) and (3.18)}

In this section we want to prove that if, given $h \leq 0$, for any $j>h(2.38)$ is satisfied, then (2.92) and (3.18) are true for $h_{i}-1 \geq h$ and (3.8) is true for $h \leq h^{\prime} \leq k \leq 0$. Combining this result with the results discussed above in Sect. 3.1 finally completes the inductive proof of the validity of (2.38) and of (2.92). Furthermore, this 
result, together with Theorem 2.1, completes the proof of convergence of the expansion for the free energy and, together with the discussion in Section 2.9, finally completes the proof of Theorem 1.1.

Given a set $P_{4}=\left\{f_{1}, f_{2}, f_{3}, f_{4}\right\}$ of four field labels, a set of sector indices $\Omega_{4}=\left\{\omega_{f} \in O_{j}: f \in P_{4}\right\}$ and a set of space-time points $\underline{\mathbf{x}}=\left\{\mathbf{x}_{f}: f \in P_{4}\right\}$ labelled by $P_{4}$, let us begin with proving that, if $U_{0}\left|h_{\beta}\right|=c_{0}$ is small enough, then

$$
\int d \mathbf{x}_{2} d \mathbf{x}_{3} d \mathbf{x}_{4}\left|\widetilde{\lambda}_{j, \Omega_{4}}(\underline{\mathbf{x}})\right| \leq C|U|, \quad j \geq h
$$

implying (2.92) for any tree $\tau \in \mathcal{T}_{h, n}, n \geq 1$. We proceed by induction. Note that for $h=0$ the bound just follows from the bounds on the kernels of the effective potential $\mathcal{V}^{(0)}$

In order to prove $(3.76)$ for $j<0$, we substitute into the definition of $\widetilde{\lambda}_{j, \Omega_{4}}$ the beta function equation in the second line of (2.36) (for notational convenience, in the following we shall drop the dependence of $\beta_{j}^{4}$ on $\left.\left(E_{j}, \lambda_{j} ; \ldots ; E_{0}, U\right)\right)$. We find

$$
\widetilde{\lambda}_{j, \Omega_{4}}(\underline{\mathbf{x}})=\mathfrak{F}_{4, j, \Omega_{4}} * \lambda_{0}(\underline{\mathbf{x}})+\sum_{j^{\prime}=j+1}^{0} \mathfrak{F}_{4, j, \Omega_{4}} * \beta_{j^{\prime}}^{4}(\underline{\mathbf{x}})
$$

where, repeating the same iterative construction leading to the tree expansion for $\mathcal{V}^{(h)}$, see $(2.87)$, and for $\beta_{h}^{2}$, see (3.4), we can represent each term in the sum in the r.h.s. of (3.77) as:

$$
\begin{aligned}
\beta_{j^{\prime}, \Omega_{4}}^{4}(\underline{\mathbf{x}}) \equiv \mathfrak{F}_{4, j, \Omega_{4}} * \beta_{j^{\prime}}^{4}(\underline{\mathbf{x}})= & \sum_{n=2}^{\infty} \sum_{\tau \in \mathcal{T}_{j^{\prime}-1, n}} \sum_{\substack{\mathbf{P} \in \mathcal{P}_{\tau} \\
\mid P_{v_{0}}=4}} \sum_{T \in \mathbf{T}} \sum_{\Omega \backslash \Omega_{v_{0}}}\left[\prod_{v \in V_{c}} \chi\left(\Omega_{v}\right)\right] \\
& \cdot \mathfrak{F}_{4, j, \Omega_{4}} * \int d\left(\mathbf{x}_{v_{0}} \backslash \underline{\mathbf{x}}\right) W_{\tau, \mathbf{P}, \Omega, T}^{(\mathrm{mod})}\left(\mathbf{x}_{v_{0}}\right) .
\end{aligned}
$$

Note that the sum in the r.h.s. begins, by construction, with the second order $(n=2)$.

In analogy with $(2.96)$, we can define $J_{j^{\prime}, n, \Omega_{4}}^{(4)}(\tau, \mathbf{P}, T)$ as the following quantity, representing a bound for the terms appearing in the sum in the r.h.s. of $(3.78)$ :

$$
\begin{aligned}
& J_{j^{\prime}, n, \Omega_{4}}^{(4)}(\tau, \mathbf{P}, T)=\sum_{\Omega \backslash \Omega_{v_{0}}} \\
& \cdot\left[\prod_{v} \chi\left(\Omega_{v}\right)\right] \int d \mathbf{x}_{2} d \mathbf{x}_{3} d \mathbf{x}_{4} \cdot\left|\mathfrak{F}_{4, j, \Omega_{4}} * \int d\left(\mathbf{x}_{v_{0}} \backslash \underline{\mathbf{x}}\right) W_{\tau, \mathbf{P}, \Omega, T}^{(\bmod )}\left(\mathbf{x}_{v_{0}}\right)\right| .
\end{aligned}
$$

Assuming inductively the bound (3.76) for $j^{\prime}>j$ and repeating the proof of Lemma 2.7, leading to the first bound in (2.118), we find:

$$
J_{j^{\prime}, n, \Omega_{4}}^{(4)}(\tau, \mathbf{P}, T) \leq(C|U|)^{n} \prod_{v \in V_{c}} \frac{1}{s_{v} !} \gamma^{\delta\left(\left|P_{v}\right|\right)\left(h_{v}-h_{v^{\prime}}\right)} .
$$


Using (3.80) into (3.78), we find $\left|\beta_{j^{\prime}, \Omega_{4}}^{4}(\underline{\mathbf{x}})\right| \leq c|U|^{2}$, so that, by (3.77)

$$
\left|\widetilde{\lambda}_{j, \Omega_{4}}(\underline{\mathbf{x}})\right| \leq C_{0}|U|+c|j||U|^{2} \leq C|U|,
$$

where in the last passage we used that $|j||U| \leq c_{0}$. This completes the proof of (2.92). The proof of (3.18) is a step by step repetition of the proof above and we do not repeat it here.

Let us now turn to the proof of (3.8). We begin with considering $j=0$. We again proceed by induction. First, we want to show the validity of (3.8) for $k=0$ and $h \leq h^{\prime} \leq 0$, that is we want to prove

$$
\sum_{\omega \in O_{h^{\prime}}} \int d\left(\mathbf{x}_{1}-\mathbf{x}_{4}\right)\left|\int d \mathbf{x}_{2} d \mathbf{x}_{3} g_{\omega}^{\left(h^{\prime}\right)}\left(\mathbf{x}_{2}-\mathbf{x}_{3}\right) \tilde{\lambda}_{0, \tilde{\Omega}_{4}}\left(\mathbf{x}_{1}, \mathbf{x}_{2}, \mathbf{x}_{3}, \mathbf{x}_{4}\right)\right| \leq C_{0}|U| \gamma^{2 h^{\prime}},
$$

where $\tilde{\Omega}_{4}$ was defined after $(3.8)$ and $\tilde{\lambda}_{0, \tilde{\Omega}_{4}}$ in (3.9). The strategy consists again in rewriting $g_{\omega}^{\left(h^{\prime}\right)}\left(\mathbf{x}_{2}-\mathbf{x}_{3}\right)$ as

$$
g_{\omega}^{\left(h^{\prime}\right)}(\mathbf{0})+\left(\mathbf{x}_{2}-\mathbf{x}_{3}\right) \sum_{\bar{\omega} \prec \omega} \int_{0}^{1} d s \partial_{\mathbf{x}} \bar{g}_{\bar{\omega}}^{\left(h^{\prime}\right)}\left(s\left(\mathbf{x}_{2}-\mathbf{x}_{3}\right)\right) .
$$

We substitute (3.83) into (3.82) and we bound the l.h.s. of (3.82) by the sum of two terms, corresponding to the two terms in (3.83). Now, the term proportional to $g_{\omega}^{\left(h^{\prime}\right)}(\mathbf{0})$ can soon be bounded as in the r.h.s. of (3.82), by Lemma 2.3 and by the estimate $(2.17)$ for $l=2$. The term corresponding to the second addend in (3.83) can be bounded as

$$
\begin{array}{r}
\sum_{\bar{\omega} \in \bar{O}_{h^{\prime}}} \sup _{\mathbf{x}}\left|\partial_{\mathbf{x}} \bar{g}_{\bar{\omega}}^{\left(h^{\prime}\right)}(\mathbf{x})\right| \int d \mathbf{x}_{2} d \mathbf{x}_{3} d \mathbf{x}_{4}\left|\mathbf{x}_{2}-\mathbf{x}_{3}\right| \cdot\left|\tilde{\lambda}_{0, \tilde{\Omega}_{4}}\left(\mathbf{x}_{1}, \mathbf{x}_{2}, \mathbf{x}_{3}, \mathbf{x}_{4}\right)\right| \\
\leq C_{0}^{\prime}|U|^{2} \gamma^{-h^{\prime}} \gamma^{3 h^{\prime}}
\end{array}
$$

and (3.82) follows.

Now, for any fixed $h^{\prime} \in[h, 0]$, we inductively suppose that (3.8) is valid for any $\bar{k}<k \leq 0$, with $\bar{k} \geq h^{\prime}$, and we prove it for $k=\bar{k}$. We insert in the r.h.s. of (3.8) the beta function equation in the second line of (2.36) and we find that (3.8) can be bounded by

$$
\begin{aligned}
& \sum_{\omega \in O_{h^{\prime}}} \int d\left(\mathbf{x}_{1}-\mathbf{x}_{4}\right)\left|\int d \mathbf{x}_{2} d \mathbf{x}_{3} g_{\omega}^{\left(h^{\prime}\right)}\left(\mathbf{x}_{2}-\mathbf{x}_{3}\right) \tilde{\lambda}_{0, \tilde{\Omega}_{4}}\left(\mathbf{x}_{1} \ldots \mathbf{x}_{4}\right)\right| \\
& +\sum_{k=\bar{k}+1}^{0} \sum_{\omega \in O_{h^{\prime}}} \int d\left(\mathbf{x}_{1}-\mathbf{x}_{4}\right)\left|\int d \mathbf{x}_{2} d \mathbf{x}_{3} g_{\omega}^{\left(h^{\prime}\right)}\left(\mathbf{x}_{2}-\mathbf{x}_{3}\right) \beta_{k, \tilde{\Omega}_{4}}^{4}\left(\mathbf{x}_{1} \ldots \mathbf{x}_{4}\right)\right|
\end{aligned}
$$

where $\beta_{k, \tilde{\Omega}_{4}}^{4}(\underline{\mathbf{x}})$ admits the same representation $(3.78)$, with $\Omega_{4}$ replaced by $\tilde{\Omega}_{4}$. The term in the first row of (3.85) can be bounded as in (3.82). Repeating the 
proof discussed in items (B.1a)-(B.3), it can be realized that the $k$-th term in the sum in the second line of (3.85) can be bounded as:

$$
\sum_{\omega \in O_{h^{\prime}}} \int d\left(\mathbf{x}_{1}-\mathbf{x}_{4}\right)\left|\int d \mathbf{x}_{2} d \mathbf{x}_{3} g_{\omega}^{\left(h^{\prime}\right)}\left(\mathbf{x}_{2}-\mathbf{x}_{3}\right) \beta_{k, \tilde{\Omega}_{4}}^{4}\left(\mathbf{x}_{1}, \mathbf{x}_{2}, \mathbf{x}_{3}, \mathbf{x}_{4}\right)\right| \leq c|U|^{2} \gamma^{2 h^{\prime}}|k| .
$$

Inserting the bounds (3.82) and (3.86) into (3.85) we find that (3.8) with $k=\bar{k}$ can be bounded by

$$
C_{0}|U| \gamma^{2 h^{\prime}}+c|U|^{2} \gamma^{2 h^{\prime}} \sum_{k=\bar{k}+1}^{0}|k| \leq C|U| \gamma^{2 h^{\prime}}|\bar{k}|
$$

where in the last passage we used that $|U||\bar{k}| \leq c_{0}$. So, (3.8) is proved in the case $j=0$.

As regards the case $j \geq 1$, we can once more proceed by induction. The term with $k=0$ and $h \leq h^{\prime} \leq 0$ and $j \geq 1$ can be bounded exactly as in (3.82), with $C_{0}$ replaced by some $C_{0, j}$. The term with $k=\bar{k}$, supposing inductively that (3.8) is valid for any $k>\bar{k}$, can be bounded by an expression analogous to (3.85), with a factor $\left|\mathbf{x}_{1}-\mathbf{x}_{4}\right|^{j}$ more appearing under the integration. Via the same strategy used to prove (3.82), we can prove that the analogue of the term in the first line of (3.85) can be bounded by $C_{0, j}|U|^{2} \gamma^{2 h^{\prime}}$; the reason for the factor $|U|^{2}$ replacing the factor $|U|$ in (3.82) is that the function $\lambda_{0}(\underline{\mathbf{x}})$ is local (hence with vanishing derivative) unless for terms of order $|U|^{2}$ coming from the ultraviolet integration.

Similarly, the analogue of the $k$-th term in the sum in the second line of (3.85) can be bounded by the r.h.s. of (3.86) (with $c$ replaced by some new constant $c_{j}$ ) times a dimensional factor $\gamma^{-j k}$ (obtained exactly as described at the end of Section 3.2 above). Substituting such bounds in the analogue of (3.85) we find that (3.8) with $k=\bar{k}$ and $j \geq 1$ can be bounded as

$$
C_{0, j}|U|^{2} \gamma^{2 h^{\prime}}+c_{j}|U|^{2} \gamma^{2 h^{\prime}} \sum_{k=\bar{k}+1}^{0}|k| \gamma^{-j k} \leq C_{j}|U|^{2}|\bar{k}| \gamma^{2 h^{\prime}-j \bar{k}}
$$

So the proof of (3.8) is complete and, together with it, the proof of convergence of the expansion for the free energy and for the two point Schwinger function is complete. In particular this concludes the proof of Theorem 1.1.

\section{Appendix A. The ultraviolet integration}

In this Appendix we prove (2.17). By definition, $W_{2 l}^{(0)}=\lim _{N \rightarrow \infty} W_{2 l}^{[0, N]}$, where $W_{2 l}^{[0, N]}$ are the kernels of the theory with effective potential $\mathcal{V}^{[0, N]}$, defined by an equation similar to $(2.14)$, with $\mathcal{E}_{1}^{T}$ replaced by the truncated expectation with 
propagator

$$
g^{(1, \leq N)}(\mathbf{x})=\sum_{j=0}^{N} g^{(1, j)}(\mathbf{x})
$$

where

$$
g^{(1, j)}(\mathbf{x})=\frac{1}{L^{2} \beta} \sum_{\mathbf{k} \in \mathcal{D}_{\beta, L}} f_{1}(\mathbf{k}) h_{j}\left(k_{0}\right) \frac{e^{-i \mathbf{k x}}}{-i k_{0}+\varepsilon_{0}(\vec{k})-\mu},
$$

with $h_{0}\left(k_{0}\right)=H_{0}\left(\left|k_{0}\right|\right)$ and $h_{j}\left(k_{0}\right)=H_{0}\left(\gamma^{-j}\left|k_{0}\right|\right)-H_{0}\left(\gamma^{-j+1}\left|k_{0}\right|\right)$.

Note that $\lim _{N \rightarrow \infty} g^{(1, \leq N)}(\mathbf{x})=g^{(+1)}(\mathbf{x})$ and that, for any integer $K \geq 0$, $g^{(1, j)}(\mathbf{x})$ satisfies the bound

$$
\left|g^{(1, j)}(\mathbf{x})\right| \leq \frac{C_{K}}{1+\left(\gamma^{j}\left|x_{0}\right|+|\vec{x}|\right)^{K}}
$$

where $\left|x_{0}\right|$ has to be thought as the distance from the origin on the one dimensional torus of size $\beta$, while $|\vec{x}|$ is the distance on the two dimensional torus of size $L^{2}$.

We associate with any propagator $g^{(1, j)}(\mathbf{x})$ a Grassmann field $\psi^{(1, j)}$ and a Gaussian integration $P\left(d \psi^{(1, j)}\right)$ with propagator $g^{(1, j)}(\mathbf{x})$. Using repeatedly the addition principle $(2.13)$, we can rewrite $\mathcal{V}^{(0)}$ as:

$$
\begin{aligned}
\mathcal{V}^{(0)}(\phi) & +L^{2} \beta F_{0} \\
& =-\lim _{N \rightarrow \infty} \log \int P\left(d \psi^{(1,0)}\right) P\left(d \psi^{(1,1)}\right) \cdots P\left(d \psi^{(1, N)}\right) e^{-V\left(\psi^{(1, \leq N)}+\phi\right)} .
\end{aligned}
$$

We can integrate iteratively the fields on scale $N, N-1, \ldots, k+1, k \geq 0$, and after each integration, using iteratively an identity like (2.14), we can rewrite the r.h.s. of (A.4) in terms of a new effective potential $\mathcal{V}^{(k, N)}$ :

$$
\begin{aligned}
(\mathrm{A} .4)= & \lim _{N \rightarrow \infty}\left\{L^{2} \beta \sum_{j=k+1}^{N} E_{j}-\right. \\
& \left.-\log \int P\left(d \psi^{(1,0)}\right) P\left(d \psi^{(1,1)}\right) \cdots P\left(d \psi^{(1, k)}\right) e^{-\mathcal{V}^{(k, N)}\left(\psi^{(1, \leq k)}+\phi\right)}\right\},
\end{aligned}
$$

with $\mathcal{V}^{(k, N)}$ admitting a representation similar to (2.16), with kernels denoted $W_{2 l}^{(k, N)}$; the constants $E_{j}$ are determined by the conditions $\mathcal{V}^{(j-1, N)}(0)=0$. Note that the possibility of rewriting the effective potential on scale 0 in the form (2.16), with $W_{2 l}^{(k, N)}$ independent of the spin labels, follows from the symmetries listed after $(2.10)$ and the remark that $P\left(d \psi^{(+1)}\right)$ itself is invariant under the same symmetries. Note also that $\mathcal{V}^{(0)}=\mathcal{V}^{(-1, N)}$ and $F_{0}=\sum_{j=0}^{\infty} E_{j}$.

By proceeding as in Section 2.6, one can see that the contribution of order $n$ in $U$ to $\mathcal{V}^{(k, N)}(\psi)$ and the corresponding kernels can be written as a sum over a family $\mathcal{T}_{(k, N), n}$ of trees $\tau$ of suitable tree values $\mathcal{V}^{(k, N)}(\tau ; \psi)$ or $W_{2 l}^{(k, N)}\left(\tau ; \mathbf{x}_{1}, \sigma_{1}, \varepsilon_{1} ; \ldots\right.$; $\left.\mathbf{x}_{2 l}, \sigma_{2 l}, \varepsilon_{2 l}\right)$, each of them computable as a product of truncated expectations $\mathcal{E}_{(1, j)}^{T}$ 
(i.e., of truncated expectations associated to the propagators $g^{(1, j)}$ of $(\mathrm{A} .2)$ ). The definition of $\mathcal{T}_{(k, N), n}$ is similar to the definition introduced in Section 2.6 above with the following modifications:

1). a tree $\tau \in \mathcal{T}_{(k, N), n}$ has vertices $v$ associated with scale labels $k+1 \leq h_{v} \leq$ $N+1$, while the root $r$ has scale $k$;

2). all the endpoints are "of type $U$ ", that is are associated to a contribution $V(\psi)$, see $(2.9)$

3). in analogy with definition 1 in $\S(3.1)$ of [BGM], we call $\chi$-vertices the vertices $v$ of $\tau$ such that the set of internal lines $\mathcal{I}_{v}$ is not empty; $V_{\chi}(\tau)$ will denote the set of all $\chi$-vertices of $\tau$.;

4). if $v$ is an endpoint, its scale is $h_{\bar{v}}+1$, if $\bar{v}$ is the $\chi$-vertex immediately preceding $v$.

The values $W_{2 l}^{(k, N)}\left(\tau ; \mathbf{x}_{1}, \sigma_{1}, \varepsilon_{1} ; \ldots ; \mathbf{x}_{2 l}, \sigma_{2 l}, \varepsilon_{2 l}\right)$ can be computed via the iterative rules described in $\S(2.4)$ of [BGM] (with some obvious modifications needed to adapt the rules to the present case) and can be bounded via the same strategy described in detail in $\S(2.7)$ of [BGM]. Using the dimensional bound (A.3), it is easy to realize that the contribution from a tree $\tau \in \mathcal{T}_{(k, N), n}$ associated with a kernel with $2 l$ external legs can be bounded, if we also fix the set $P_{v}$ of external lines in each vertex and call $W_{2 l}^{(k, N)}(\tau ; \mathbf{P})$ the corresponding kernel, as:

$$
\begin{aligned}
& \frac{1}{L^{2} \beta} \int d \mathbf{x}_{1} \ldots d \mathbf{x}_{2 l} \mid W_{2 l}^{(k, N)}\left(\tau ; \mathbf{P} ; \mathbf{x}_{1}, \sigma_{1}, \varepsilon_{1} ; \ldots ; \mathbf{x}_{2 l}, \sigma_{2 l}, \varepsilon_{2 l}\right) \mid \\
& \leq C^{n}|U|^{n} \gamma^{-k\left(n-1+n^{\mathrm{tad}}\right)} \prod_{v \in V_{\chi}} \gamma^{-\left(h_{v}-h_{v^{\prime}}\right)\left(n_{v}-1+n_{v}^{\mathrm{tad}}\right)}
\end{aligned}
$$

where $v^{\prime}$ is the $\chi$-vertex immediately preceding $v$ on $\tau, n_{v}$ is the number of endpoints following $v$ on $\tau, n^{\text {tad }}$ is the total number of tadpoles on $\tau$ (i.e., the number of trivial $\chi$-vertices $v$ with $n_{v}=1$ ) and $n_{v}^{\text {tad }}$ is the number of tadpoles following $v$ on $\tau$.

Remark. In deriving (A.6) an explicit computation of the tadpole contributions is needed. In particular one has to realize that a parity cancellation implies that the tadpole contribution associated with the subtree rooted on a trivial $\chi$-vertex $v$ of scale $h_{v}$ with $n_{v}=1$ can be bounded by $C \gamma^{-h_{v}}$ (instead of the naive dimensional bound $C$ ).

Note that the "internal dimensions" $\left(n_{v}-1+n_{v}^{\text {tad }}\right)$ are all $>1$, so that the sum over $\mathbf{P}$ and $\tau$ of (A.6) gives rise in the usual way to a bound $L^{2} \beta(C|U|)^{n}$. The insertion in the integral of the factor $\prod_{1 \leq i<j \leq 2 l}\left|\vec{x}_{i}-\vec{x}_{j}\right|^{m_{i j}}$ will produce the same type of bound, with $C^{n+m}, m=\sum m_{i j}$, in place of $C^{n}$, so that (2.17) follows, for $U$ small enough. 


\section{Appendix B. Proof of Lemma 3.1}

Given $h \leq 0$ and an isotropic sector index of scale $h, \bar{\omega} \in \bar{O}_{h}$, let us call $\bar{S}_{h, \bar{\omega}}$ the corresponding isotropic s-sector (here we choose a notation as close as possible to that introduced in [BGM] for the anisotropic sectors, see (2.72) of [BGM]).

Let assign $\bar{\omega}_{1} \equiv \bar{\omega}_{f_{1}} \in \bar{O}_{h}$ and let us call $A_{h}\left(\bar{\omega}_{1} ; \bar{\omega}_{2}, \bar{\omega}_{3}, \bar{\omega}_{4}\right)$ the set of sequences $\left(\bar{\omega}_{2}, \bar{\omega}_{3}, \bar{\omega}_{4}\right)$ in $\bar{O}_{h} \times \bar{O}_{h} \times \bar{O}_{h}$ such that

$$
\text { there exists a sequence of vectors }\left(\vec{k}_{1}, \vec{k}_{2}, \vec{k}_{3}, \vec{k}_{4}\right) \text { s.t. }
$$

$$
\vec{k}_{i} \in \bar{S}_{h, \bar{\omega}_{i}} \text { and } \sum_{i=1}^{4} \vec{k}_{i}=\overrightarrow{0} .
$$

We want to prove that

$$
\left|A_{h}\left(\bar{\omega}_{1} ; \bar{\omega}_{2}, \bar{\omega}_{3}, \bar{\omega}_{4}\right)\right| \leq C \gamma^{-h}|h|,
$$

for some constant $C>0$. Let $\theta_{i}$ be the center of the $\theta$-interval which the polar angle of $\vec{p}$ has to belong to, if $\vec{p} \in \bar{S}_{h, \bar{\omega}_{i}}$. For any pair $(i, j), i, j=1,2,3,4$, we define:

$$
\phi_{i, j}=\min \left\{\left\|\theta_{i}-\theta_{j}\right\|, \pi-\left\|\theta_{i}-\theta_{j}\right\|\right\},
$$

where $\|\cdot\|$ is the distance on the torus. By a reordering of the sectors, we can always impose the condition:

$$
\max \left\{\phi_{1,3}, \phi_{1,4}\right\} \leq \phi_{1,2} .
$$

In fact, calling $A_{h}^{*}\left(\bar{\omega}_{1} ; \bar{\omega}_{2}, \bar{\omega}_{3}, \bar{\omega}_{4}\right)$ the subset of $A_{h}\left(\bar{\omega}_{1} ; \bar{\omega}_{2}, \bar{\omega}_{3}, \bar{\omega}_{4}\right)$ with $\bar{\omega}_{2}, \bar{\omega}_{3}, \bar{\omega}_{4}$ satisfying condition (B.3), it holds:

$$
\left|A_{h}\left(\bar{\omega}_{1} ; \bar{\omega}_{2}, \bar{\omega}_{3}, \bar{\omega}_{4}\right)\right| \leq 3\left|A_{h}^{*}\left(\bar{\omega}_{1} ; \bar{\omega}_{2}, \bar{\omega}_{3}, \bar{\omega}_{4}\right)\right|
$$

So, we describe how to bound $\left|A_{h}^{*}\left(\bar{\omega}_{1} ; \bar{\omega}_{2}, \bar{\omega}_{3}, \bar{\omega}_{4}\right)\right|$. With no loss of generality, we shall assume $\phi_{1,2} \leq \pi / 2$ (the case $\phi_{1,2} \geq \pi / 2$ can be reduced to $\phi_{1,2} \leq \pi / 2$, by the reflection symmetry of the Fermi surface).

We first note that, given any positive constant $\kappa_{0}$, if we define

$$
\mathcal{A}_{<}\left(\kappa_{0}\right)=\left\{\left(\bar{\omega}_{2}, \bar{\omega}_{3}, \bar{\omega}_{4}\right) \in A_{h}^{*}\left(\bar{\omega}_{1} ; \bar{\omega}_{2}, \bar{\omega}_{3}, \bar{\omega}_{4}\right):\left|\phi_{1,2}\right| \leq \pi \kappa_{0} \gamma^{\frac{h}{2}}\right\},
$$

we have:

$$
\left|\mathcal{A}_{<}\left(\kappa_{0}\right)\right| \leq 96 \kappa_{0}^{2} \gamma^{-h}
$$

In fact, for any choice of $\phi_{1,2}=\pi k \gamma^{h},|k|=0,1, \ldots,\left[\kappa_{0} \gamma^{-\frac{h}{2}}\right] \equiv N\left(\kappa_{0}\right)$, by condition (B.3) we have that $\bar{\omega}_{3}$ can be chosen at most in $|k|+2$ different ways. Finally, once $\bar{\omega}_{2}$ and $\bar{\omega}_{3}$ are fixed, by momentum conservation $\bar{\omega}_{4}$ is fixed in a finite number of sectors (and it is easy to realize that such number is $\leq 8$ ). Then $\left|\mathcal{A}_{<}\left(\kappa_{0}\right)\right|$ is bounded by $8 \sum_{k=-N\left(\kappa_{0}\right)}^{N\left(\kappa_{0}\right)}(|k|+2)=8\left[N\left(\kappa_{0}\right)+2\right]\left[N\left(\kappa_{0}\right)+3\right] \leq 96 \kappa_{0}^{2} \gamma^{-h}$ and (B.6) follows.

Then, in order to prove (B.1), it is sufficient to prove that a similar bound is valid for the set $\mathcal{A}_{>}\left(\kappa_{0}\right) \equiv A_{h}^{*}\left(\bar{\omega}_{1} ; \bar{\omega}_{2}, \bar{\omega}_{3}, \bar{\omega}_{4}\right) \backslash \mathcal{A}_{<}\left(\kappa_{0}\right)$. We start with computing the number $N$ of pairs of angles $\left(\theta_{3}, \theta_{4}\right)$ compatible with a given choice of $\theta_{1}, \theta_{2}$ s.t. $\pi / 2 \geq \phi_{1,2} \geq \pi \kappa_{0} \gamma^{h / 2}$. Since $\vec{p}_{F}^{(h)}(\theta)=-\vec{p}_{F}^{(h)}(\theta+\pi)$, if we write $\theta_{3}, \theta_{4}$ in the 
form: $\theta_{i}+\pi+\pi n_{i} \gamma^{h}, i=1,2$, it is clear that $N$ can be bounded by twice the number of pairs $\vec{n}=\left(n_{1}, n_{2}\right)$ of integers compatible with the condition

$$
\sum_{i=1}^{2}\left[\vec{p}_{F}^{(h)}\left(\theta_{i}+\pi n_{i} \gamma^{h}\right)-\vec{p}_{F}^{(h)}\left(\theta_{i}\right)\right]=\gamma^{h} \vec{r} \quad \text { with } \quad|\vec{r}| \leq R,
$$

for some $O(1)$ constant $R$. (B.7) can be rewritten in the form:

$$
\vec{r}=\vec{f}(\vec{n}) \equiv \gamma^{-h} \sum_{i=1}^{2}\left[\vec{p}_{F}^{(h)}\left(\theta_{i}+\pi n_{i} \gamma^{h}\right)-\vec{p}_{F}^{(h)}\left(\theta_{i}\right)\right],
$$

with $\vec{f}$ twice differentiable. We now want to apply Dini's implicit function theorem in order to invert (B.8) in a neighborhood of $\vec{n}=\vec{r}=\overrightarrow{0}$ (that is an "unperturbed" solution to (B.8)). Let $A \stackrel{\text { def }}{=}(D f(\underline{0}))^{-1}$ and $\|A\|$ its norm. A simple application of Dini's Theorem implies that, if $\vec{n}$ varies in a ball $B_{\rho}(\overrightarrow{0})$ around $\overrightarrow{0}$ of radius $\rho$ so small that

$$
\|D \vec{f}(\vec{n})-D \vec{f}(\overrightarrow{0})\| \leq \frac{1}{4\|A\|}, \quad \forall \vec{n} \in B_{\rho}(\overrightarrow{0})
$$

and $\sigma<\rho /(2\|A\|)$, then for any $\vec{r} \in B_{\sigma}(\overrightarrow{0})$ we can invert (C.6) as

$$
\vec{n}=\vec{g}(\vec{r}), \quad \vec{r} \in B_{\sigma}(\overrightarrow{0}), \quad \sigma<\frac{\rho}{2\|A\|},
$$

with $\vec{g}$ twice differentiable and such that the image of $B_{\sigma}(\overrightarrow{0})$ through $\vec{g}$ is contained in $B_{\rho}(\overrightarrow{0})$. In order to have the condition $|\vec{r}| \leq R$ in (B.7) verified together with the condition $|\vec{r}| \leq \frac{\rho}{2\|A\|}$ in (B.10), we can choose

$$
\rho \stackrel{\text { def }}{=} 2\|A\| R \text {. }
$$

We now want to compute $\|A\|$ and check (B.9). Using (7.3) and (7.6) of [BGM] and calling $s_{i}^{\prime} \equiv s^{\prime}\left(\theta_{i}+\pi \gamma^{h} n_{i}\right)$, we see that the Jacobian of $\vec{f}(\vec{n})$, in the basis $\vec{n}_{h}\left(\theta_{1}\right), \vec{\tau}_{h}\left(\theta_{1}\right)$, is:

$$
D \vec{f}(\vec{n})=\pi\left(\begin{array}{cc}
s_{1}^{\prime} \sin \left(\alpha\left(\theta_{1}\right)-\alpha\left(\theta_{1}+\pi \gamma^{h} n_{1}\right)\right) & s_{2}^{\prime} \sin \left(\alpha\left(\theta_{1}\right)-\alpha\left(\theta_{2}+\pi \gamma^{h} n_{2}\right)\right) \\
s_{1}^{\prime} \cos \left(\alpha\left(\theta_{1}\right)-\alpha\left(\theta_{1}+\pi \gamma^{h} n_{1}\right)\right) & s_{2}^{\prime} \cos \left(\alpha\left(\theta_{1}\right)-\alpha\left(\theta_{2}+\pi \gamma^{h} n_{2}\right)\right)
\end{array}\right)
$$

so that $\operatorname{det} D \vec{f}(\overrightarrow{0})=\pi^{2} s^{\prime}\left(\theta_{1}\right) s^{\prime}\left(\theta_{2}\right) \sin \left(\alpha\left(\theta_{2}\right)-\alpha\left(\theta_{1}\right)\right)$. Hence, recalling the definition of $A$ (see the lines preceding (B.9)) and the property $c_{1}\left\|\theta_{1}-\theta_{2}\right\| \leq$ $\left\|\alpha\left(\theta_{1}\right)-\alpha\left(\theta_{2}\right)\right\| \leq c_{2}\left\|\theta_{1}-\theta_{2}\right\|$ (see (7.5) of [BGM] and Section 2 of the present paper), we have:

$$
\frac{C_{1}}{\left|\sin \left(\theta_{1}-\theta_{2}\right)\right|} \leq\|A\| \leq \frac{C_{2}}{\left|\sin \left(\theta_{1}-\theta_{2}\right)\right|} .
$$

Using the bound (B.13), we now want to check that the definition of $\rho$ in (B.11) is compatible with (B.9). We first note that, since $s^{\prime}(\theta)$ is differentiable, the l.h.s. 
of (B.9) is bounded by $c \gamma^{h}|\vec{n}| \leq c \gamma^{h} \rho=2 c R\|A\| \gamma^{h}$, for some $O(1)$ constant $c>0$. So, (B.9) holds if $8 c R\|A\|^{2} \gamma^{h} \leq 1$ and, by (B.13), this is surely true if

$$
8 c R C_{2}^{2} \frac{\gamma^{h}}{\sin ^{2}\left(\theta_{1}-\theta_{2}\right)} \leq 1 \text {. }
$$

We now recall that, since we are assuming that $\left\|\theta_{1}-\theta_{2}\right\| \geq \pi \kappa_{0} \gamma^{h / 2}$, it holds: $\left|\sin \left(\theta_{1}-\theta_{2}\right)\right| \geq \frac{2}{\pi}\left\|\theta_{1}-\theta_{2}\right\| \geq 2 \kappa_{0} \gamma^{h / 2}$. This means that (B.14) is satisfied if $\kappa_{0}$ is big enough, that is if

$$
\kappa_{0}^{2} \geq 2 c R C_{2}^{2}
$$

for instance we can choose $\kappa_{0} \equiv C_{2} \sqrt{2 c R}$.

With these choices, we finally have that (B.8) can be inverted into (B.10) and, if $|\vec{r}| \leq R$, then $|\vec{n}| \leq 2\|A\| R \leq \frac{2 C_{2} R}{\left|\sin \left(\theta_{1}-\theta_{2}\right)\right|}$. This means in particular that, for any choice of $\theta_{2}=\theta_{1}+\pi k \gamma^{h},\left[\kappa_{0} \gamma^{-\frac{h}{2}}\right] \leq|k| \leq\left[\frac{\gamma^{-h}}{2}\right], \bar{\omega}_{3}$ can be chosen in at most $\frac{4 C_{2} R}{\left|\sin \left(\pi k \gamma^{h}\right)\right|}$ different ways. Once both $\bar{\omega}_{2}$ and $\bar{\omega}_{3}$ are chosen, by momentum conservation $\bar{\omega}_{4}$ is essentially fixed (it can be chosen in finite number of ways, and one can realize that this number is $\leq 8)$. Then the number of elements of $\mathcal{A}_{>}$can be bounded as:

$$
\left|\mathcal{A}_{>}\left(\kappa_{0}\right)\right| \leq 2 \sum_{k=\left[\kappa_{0} \gamma^{-\frac{h}{2}}\right]}^{\left[\frac{\gamma^{-h}}{2}\right]} \frac{16 C_{2} R}{k \gamma^{h}} \leq\left[16 C_{2} R \log \gamma\right] \gamma^{-h}|h| .
$$

Combining (B.16) with (B.6) we get (B.1).

\section{Appendix C. Improved proofs of geometric lemmas}

In this Appendix we want to prove the following lemma (called Sector Counting Lemma).

Lemma C.1. Let $h^{\prime}, h, L$ be integers such that $h^{\prime} \leq h \leq 0$ and $L \geq 4$. Given $\omega_{1}^{\left(h^{\prime}\right)}, \ldots, \omega_{L}^{\left(h^{\prime}\right)} \in O_{h^{\prime}}$ and $\omega_{2}^{(h)}, \ldots, \omega_{L}^{(h)} \in O_{h}$, let us define $\Omega_{L}^{\left(h^{\prime}\right)}=\left\{\omega_{i}^{\left(h^{\prime}\right)}\right\}_{i=1}^{L}$, $\Omega_{L-1}^{\left(h^{\prime}\right)}=\left\{\omega_{i}^{\left(h^{\prime}\right)}\right\}_{i=2}^{L}$ and $\Omega_{L-1}^{(h)}=\left\{\omega_{i}^{(h)}\right\}_{i=2}^{L}$. Then, for any choice of $\omega_{1}^{\left(h^{\prime}\right)}$, it is

$$
\sum_{\Omega_{L-1}^{\left(h^{\prime}\right)} \prec \Omega_{L-1}^{(h)}} \chi\left(\Omega_{L}^{\left(h^{\prime}\right)}\right) \leq c^{L} \gamma^{\frac{1}{2}\left(h-h^{\prime}\right)(L-3)},
$$

uniformly in $\omega_{1}^{\left(h^{\prime}\right)}$ and in $\Omega_{L-1}^{(h)}$. The symbol $\prec$ must be interpreted as explained after (2.108).

In $[\mathrm{BGM}]$ a slightly different version of Lemma C.1 was proved, see Lemma (3.1) of $[\mathrm{BGM}]$. The main differences between the Lemma C.1 and the one proved in [BGM] are the following: in [BGM] the case of a fixed $C^{\infty}$ Fermi surface was considered, while here we are studying the case of a $C^{2}$ Fermi surface, changing step by step of $O\left(|U||h| \gamma^{2 h}\right)$. 
By a critical rereading of the proof of Lemma 3.1 of [BGM], see Section 7 of $[\mathrm{BGM}]$, it can be realized that the proof in $[\mathrm{BGM}]$ can be easily adapted to the case of a $C^{\infty}$ Fermi surface, changing step by step of $O\left(|U||h| \gamma^{2 h}\right)$. In order to see this it must be taken into account that, as already noted after (2.89), given an s-sector $S_{h, \omega}$ on scale $h$, there are exactly $\gamma^{\frac{1}{2}\left(h-h^{\prime}\right)}$ s-sectors on scale $h^{\prime}$ strictly contained into it, and their centers $\theta_{h^{\prime}, \omega^{\prime}}$ are independent of the specific shape of the Fermi surface. Moreover the fact that $\vec{p}_{F}^{\left(h^{\prime}\right)}(\theta)$ is convex (uniformly in $h^{\prime}$ ) must be used. Keeping these two remarks in mind, the proof of Lemma C.1 above in the case of a $C^{\infty}$ Fermi surface, changing step by step of $O\left(|U||h| \gamma^{2 h}\right)$, is easily obtained by replacing any $\vec{p}_{F}(\theta)$ appearing in the proof in Section 7 of [BGM] by $\vec{p}_{F}^{\left(h^{\prime}\right)}(\theta)$.

While the fact that the Fermi surface was fixed was not really used in the proof in Section 7 of [BGM], the fact that it was chosen as a $C^{\infty}$ curve was used here and there and it is not so straightforward to adapt the proof of [BGM] to the present $C^{2}$ case. However by a careful rereading of Section 7 of [BGM], it can be realized that the only places where the $C^{2}$ regularity of $\vec{p}_{F}(\theta)$ was used were the proofs of Lemma 7.1 and of Lemma 7.5, where some error terms were bounded by the third derivatives of $\vec{p}_{F}(\theta)$. In this section we want to reproduce the proofs of these two lemmas in a more careful way and the result will be that the proofs also work in the case that the Fermi surface is $C^{2}$, and not more regular than this. This in particular implies the validity of Lemma C.1 above.

In the following we refer for notation to Section 7 of $[\mathrm{BGM}]$, but we shall drop everywhere the dependence on $e$. Moreover, all the quantities $\vec{p}_{F}(\theta), u(\theta)$, $s(\theta), \vec{n}(\theta), \vec{\tau}(\theta)$ appearing below do depend on $h^{\prime}: \vec{p}_{F}(\theta)$ must be interpreted as equal to $\vec{p}_{F}^{\left(h^{\prime}\right)}(\theta)$ and all the other quantities are obtained from $\vec{p}_{F}^{\left(h^{\prime}\right)}(\theta)$ via the definitions in Section 7 of [BGM]. We chose to drop the dependence on $h^{\prime}$ in order to unify the notation with that of Section 7 of $[\mathrm{BGM}]$.

\section{C.1. Proof of Lemma 7.1 of [BGM]}

We want to prove that $0<c_{1} \leq \alpha^{\prime}(\theta) \leq c_{2}$, where, if $\cos \alpha=\left(u \cos \theta+u^{\prime} \sin \theta\right) / s^{\prime}$ $>0$,

$$
\alpha(\theta)=f(\theta) \equiv \arcsin \left\{\frac{1}{s^{\prime}(\theta)}\left[u(\theta) \sin \theta-u^{\prime}(\theta) \cos \theta\right]\right\},
$$

otherwise $\alpha(\theta)=\pi-f(\theta)$. By explicitly performing the derivative, we find:

$$
f^{\prime}(\theta)=\frac{-\frac{s^{\prime \prime}(\theta)}{s^{\prime}(\theta)}\left[u(\theta) \sin \theta-u^{\prime}(\theta) \cos \theta\right]+\left[2 u^{\prime}(\theta) \sin \theta+\left(u(\theta)-u^{\prime \prime}(\theta)\right) \cos \theta\right]}{s^{\prime}(\theta) \sqrt{1-\frac{1}{\left(s^{\prime}(\theta)\right)^{2}}\left[u(\theta) \sin \theta-u^{\prime}(\theta) \cos \theta\right]^{2}}} .
$$


Using that $s^{\prime}(\theta)=\sqrt{u^{2}(\theta)+\left(u^{\prime}(\theta)\right)^{2}}$, we see that the denominator in (C.3) is equal to

$$
\begin{array}{r}
\sqrt{u^{2}(\theta)+\left(u^{\prime}(\theta)\right)^{2}-u^{2}(\theta) \sin ^{2} \theta-\left(u^{\prime}(\theta)\right)^{2} \cos ^{2} \theta+2 u(\theta) u^{\prime}(\theta) \sin \theta \cos \theta} \\
=\left|u(\theta) \cos \theta+u^{\prime}(\theta) \sin \theta\right|=s^{\prime}(\theta)|\cos \alpha(\theta)|
\end{array}
$$

Using that $s^{\prime \prime}(\theta)=\frac{u u^{\prime}+u^{\prime} u^{\prime \prime}}{\sqrt{u^{2}+\left(u^{\prime}\right)^{2}}}$, we can rewrite the numerator in (C.3) as:

$$
\begin{aligned}
& {\left[-\frac{\left(u u^{\prime}+u^{\prime} u^{\prime \prime}\right)}{u^{2}+\left(u^{\prime}\right)^{2}}\left(u \sin \theta-u^{\prime} \cos \theta\right)+2 u^{\prime} \sin \theta+\left(u-u^{\prime \prime}\right) \cos \theta\right]} \\
& =\frac{1}{\left[u^{2}+\left(u^{\prime}\right)^{2}\right]}\left[-\left(u u^{\prime}+u^{\prime} u^{\prime \prime}\right)\left(u \sin \theta-u^{\prime} \cos \theta\right)\right. \\
& \left.+\left(2 u^{\prime} \sin \theta+\left(u-u^{\prime \prime}\right) \cos \theta\right)\left(u^{2}+\left(u^{\prime}\right)^{2}\right)\right] \\
& =\frac{1}{\left[u^{2}+\left(u^{\prime}\right)^{2}\right]}\left[\sin \theta\left(2\left(u^{\prime}\right)^{3}+u^{2} u^{\prime}-u u^{\prime} u^{\prime \prime}\right)\right. \\
& \left.+\cos \theta\left(2 u\left(u^{\prime}\right)^{2}+u^{3}-u^{2} u^{\prime \prime}\right)\right] \\
& =\frac{1}{\left[u^{2}+\left(u^{\prime}\right)^{2}\right]}\left(u^{\prime} \sin \theta+u \cos \theta\right)\left(2\left(u^{\prime}\right)^{2}+u^{2}-u u^{\prime \prime}\right) \\
& =\frac{s^{\prime}(\theta)^{3}}{r(\theta)\left[u^{2}+\left(u^{\prime}\right)^{2}\right]}\left(u^{\prime} \sin \theta+u \cos \theta\right)=\frac{s^{\prime}(\theta)^{2} \cos \alpha(\theta)}{r(\theta)} \text {, }
\end{aligned}
$$

where, in the last identity, we used the identity $\left[2\left(u^{\prime}\right)^{2}+u^{2}-u u^{\prime \prime}\right]=\left(s^{\prime}(\theta)\right)^{3} / r(\theta)$, which is an easy consequence of (7.3) and (7.4) of [BGM]. It follows that $\alpha^{\prime}(\theta)=$ $s^{\prime}(\theta) / r(\theta)$, that is bounded away from 0 , by hypothesis.

\section{C.2. Proof of Lemma 7.5 of [BGM]}

With the definitions introduced in the proof of Lemma 7.5, we can rewrite (7.24) of $[\mathrm{BGM}]$ as:

$$
\begin{aligned}
\left(\begin{array}{c}
\widetilde{r}_{1} \\
\widetilde{r}_{2}
\end{array}\right) & =\underline{f}\left(x_{1}, x_{2}\right) \\
& =\frac{1}{\eta}\left(\begin{array}{c}
\phi^{-1}\left[\left[\vec{p}_{F}\left(\bar{\theta}_{1 \eta}\right)-\vec{p}_{F}\left(\bar{\theta}_{1}\right)\right] \cdot \vec{n}\left(\bar{\theta}_{1}\right)+\left[\vec{p}_{F}\left(\bar{\theta}_{2 \eta}\right)-\vec{p}_{F}\left(\bar{\theta}_{2}\right)\right] \cdot \vec{n}\left(\bar{\theta}_{1}\right)\right] \\
{\left[\vec{p}_{F}\left(\bar{\theta}_{1 \eta}\right)-\vec{p}_{F}\left(\bar{\theta}_{1}\right)\right] \cdot \vec{\tau}\left(\bar{\theta}_{1}\right)+\left[\vec{p}_{F}\left(\bar{\theta}_{2 \eta}\right)-\vec{p}_{F}\left(\bar{\theta}_{2}\right)\right] \cdot \vec{\tau}\left(\bar{\theta}_{1}\right)}
\end{array}\right)
\end{aligned}
$$

where $\bar{\theta}_{i \eta} \equiv \bar{\theta}_{i}+\eta x_{i}$.

The equation $\underline{\widetilde{r}}=\underline{f}(\underline{x})$ admits an unperturbed solution $\underline{0}=\underline{f}(\underline{0})$ and we want to apply Dini's theorem to invert the equation as $\underline{x}=\underline{g}(\underline{\widetilde{r}})$ in a neighborhood of $\underline{\widetilde{r}}=\underline{0}$. 
The Jacobian matrix of $\underline{f}$ is:

$$
D \underline{f}(\underline{x})=\left(\begin{array}{cc}
\phi^{-1} \vec{n}\left(\bar{\theta}_{1}\right) \partial_{\theta} \vec{p}_{F}\left(\bar{\theta}_{1 \eta}\right) & \phi^{-1} \vec{n}\left(\bar{\theta}_{1}\right) \partial_{\theta} \vec{p}_{F}\left(\bar{\theta}_{2 \eta}\right) \\
\vec{\tau}\left(\bar{\theta}_{1}\right) \partial_{\theta} \vec{p}_{F}\left(\bar{\theta}_{1 \eta}\right) & \vec{\tau}\left(\bar{\theta}_{1}\right) \partial_{\theta} \vec{p}_{F}\left(\bar{\theta}_{2 \eta}\right)
\end{array}\right)
$$

and, using (7.3) of [BGM], we can rewrite:

$$
D \underline{f}(\underline{x})=\left(\begin{array}{cc}
s^{\prime}\left(\bar{\theta}_{1 \eta}\right) \phi^{-1} \vec{n}\left(\bar{\theta}_{1}\right) \vec{\tau}\left(\bar{\theta}_{1 \eta}\right) & s^{\prime}\left(\bar{\theta}_{2 \eta}\right) \phi^{-1} \vec{n}\left(\bar{\theta}_{1}\right) \vec{\tau}\left(\bar{\theta}_{2 \eta}\right) \\
s^{\prime}\left(\bar{\theta}_{1 \eta}\right) \vec{\tau}\left(\bar{\theta}_{1}\right) \vec{\tau}\left(\bar{\theta}_{1 \eta}\right) & s^{\prime}\left(\bar{\theta}_{2 \eta}\right) \vec{\tau}\left(\bar{\theta}_{1}\right) \vec{\tau}\left(\bar{\theta}_{2 \eta}\right)
\end{array}\right) .
$$

By using (7.6) of [BGM], we can also write:

$$
\begin{aligned}
& D \underline{f}(\underline{x})= \\
& \qquad\left(\begin{array}{lc}
-\frac{s^{\prime}\left(\bar{\theta}_{1 \eta}\right)}{\phi} \sin \left(\alpha\left(\bar{\theta}_{1 \eta}\right)-\alpha\left(\bar{\theta}_{1}\right)\right) & -\frac{s^{\prime}\left(\bar{\theta}_{2 \eta}\right)}{\phi} \sin \left(\alpha\left(\bar{\theta}_{2 \eta}\right)-\alpha\left(\bar{\theta}_{1}\right)\right) \\
s^{\prime}\left(\bar{\theta}_{1 \eta}\right) \cos \left(\alpha\left(\bar{\theta}_{1 \eta}\right)-\alpha\left(\bar{\theta}_{1}\right)\right) & s^{\prime}\left(\bar{\theta}_{2 \eta}\right) \cos \left(\alpha\left(\bar{\theta}_{2 \eta}\right)-\alpha\left(\bar{\theta}_{1}\right)\right)
\end{array}\right) .
\end{aligned}
$$

In particular, the Jacobian determinant at $\underline{x}=\underline{0}$ is

$$
\operatorname{det} D \underline{f}(\underline{0})=s^{\prime}\left(\bar{\theta}_{1}\right) s^{\prime}\left(\bar{\theta}_{2}\right) \phi^{-1} \sin \left(\alpha\left(\bar{\theta}_{2}\right)-\alpha\left(\bar{\theta}_{1}\right)\right),
$$

which is bounded above and below by $O(1)$ constants. As a consequence, there exists $A \stackrel{\text { def }}{=}(D \underline{f}(\underline{0}))^{-1}$ and its norm $\|A\|$ is a $O(1)$ constant.

Now, a simple application of Dini's Theorem implies that, if $\rho$ is so small that

$$
\|D \underline{f}(\underline{x})-D \underline{f}(\underline{0})\| \leq \frac{1}{4\|A\|}, \quad \forall \underline{x} \in B_{\rho}(\underline{0})
$$

and $r<\rho /(2\|A\|)$, then we can invert uniquely (C.6) for any $\underline{\widetilde{r}} \in B_{r}(\underline{0})$ as

$$
\underline{x}=\underline{g}(\underline{\widetilde{r}})
$$

and the image of $B_{r}(\underline{0})$ through $g$ is contained in $B_{\rho}(\underline{0})$. So, let us compute $\|D \underline{f}(\underline{x})-D \underline{f}(\underline{0})\|$ in our case. We have that $D \underline{f}(\underline{x})-D \underline{f}(\underline{0})$ is given by:

$$
\left(\begin{array}{cc}
-\phi^{-1} s^{\prime}\left(\bar{\theta}_{1 \eta}\right) \cdot & -\phi^{-1} s^{\prime}\left(\bar{\theta}_{2 \eta}\right) \sin \left(\alpha\left(\bar{\theta}_{2 \eta}\right)-\alpha\left(\bar{\theta}_{1}\right)\right) \\
\cdot \sin \left(\alpha\left(\bar{\theta}_{1 \eta}\right)-\alpha\left(\bar{\theta}_{1}\right)\right) & +\phi^{-1} s^{\prime}\left(\bar{\theta}_{2}\right) \sin \left(\alpha\left(\bar{\theta}_{2}\right)-\alpha\left(\bar{\theta}_{1}\right)\right) \\
s^{\prime}\left(\bar{\theta}_{1 \eta}\right) \cdot \bar{\theta}^{\prime}\left(\bar{\theta}_{2 \eta}\right) \cos \left(\alpha\left(\bar{\theta}_{2 \eta}\right)-\alpha\left(\bar{\theta}_{1}\right)\right) \\
\cdot \cos \left(\alpha\left(\bar{\theta}_{1 \eta}\right)-\alpha\left(\bar{\theta}_{1}\right)\right)-s^{\prime}\left(\bar{\theta}_{1}\right) & -s^{\prime}\left(\bar{\theta}_{2}\right) \cos \left(\alpha\left(\bar{\theta}_{2}\right)-\alpha\left(\bar{\theta}_{1}\right)\right)
\end{array}\right)
$$

and its norm is bounded by $C\left(c_{2}+\eta\right)|\underline{x}|$, where $c_{2}$ was defined in Lemma 7.5 of [BGM] and $C$ is constant depending only on the second derivative of $s(\theta)$ and the first derivative of $\alpha(\theta)$, that is at most on second derivatives of $u(\theta)$. Now, choosing $c_{2}$ sufficiently small, we can say that for (C.11) is satisfied with $\rho=1$ and (C.12) holds for any $|\underline{\widetilde{r}}| \leq c$, where $c$ is a suitable $O(1)$ constant. 


\section{Appendix D. Proof of Lemma 2.6}

Consider the sector sum (2.117), where the sum runs over the sector indices in $\Omega \backslash \Omega_{\text {ext }}^{(F)}$ and, given $f_{1}, \ldots, f_{F} \in P_{v_{0}}$, we defined $\Omega_{\text {ext }}^{(F)}=\left\{\omega_{f_{i}}\right\}_{i=1}^{F}$. We want to prove that, if $F=3$ or $F=5$, the sum in (2.117) can be bounded by the expression in the r.h.s. of $(2.103)$, i.e., by the bound for the sector sum with $F=1$, times a dimensional gain of $\gamma^{h / 2}$ or $\gamma^{h}$, depending whether $F=3$ or $F=5$.

First of all, let us note that, if $\tau$ is a trivial tree or $\tau \in \mathcal{T}_{h, 1}$ and $\left|P_{v_{0}}\right|=4$, the bound we want to prove is obvious. In fact, in these cases only $F=3$ is allowed and, if $\omega_{1}, \ldots, \omega_{4} \in O_{h}$ and $\Omega_{4}=\left\{\omega_{1}, \ldots, \omega_{4}\right\}$, it is

$$
\sum_{\omega_{1} \in O_{h}} \chi\left(\Omega_{4}\right) \leq c
$$

simply by momentum conservation. Another simple consequence of momentum conservation is the following.

Lemma D.1. Let $h^{\prime}, h, L, F$ be integers such that $h^{\prime} \leq h \leq 0, L \geq 4$ and $1 \leq$ $F \leq L-1$. Given $\omega_{1}^{\left(h^{\prime}\right)}, \ldots, \omega_{L}^{\left(h^{\prime}\right)} \in O_{h^{\prime}}$ and $\omega_{1}^{(h)}, \ldots, \omega_{F}^{(h)} \in O_{h}$, let us define $\Omega_{L}^{\left(h^{\prime}\right)}=\left\{\omega_{i}^{\left(h^{\prime}\right)}\right\}_{i=1}^{L}, \Omega_{L-F}^{\left(h^{\prime}\right)}=\left\{\omega_{i}^{\left(h^{\prime}\right)}\right\}_{i=F+1}^{L}$ and $\Omega_{L-F}^{(h)}=\left\{\omega_{i}^{(h)}\right\}_{i=F+1}^{L}$. Then, for any choice of $\omega_{1}^{\left(h^{\prime}\right)}, \ldots, \omega_{F}^{\left(h^{\prime}\right)} \in O_{h^{\prime}}$ it is

$$
\sum_{\Omega_{L-F}^{\left(h^{\prime}\right)} \prec \Omega_{L-F}^{(h)}} \chi\left(\Omega_{L}^{\left(h^{\prime}\right)}\right) \leq c^{L} \gamma^{\frac{1}{2}\left(h-h^{\prime}\right)(L-F-1)} .
$$

Proof. If $F=L-1$ the statement of the lemma is a simple consequence of momentum conservation. If $F<L-1$, then we can bound (D.2) by

$$
\sum_{\substack{\left(h^{\prime}\right) \\ \omega_{F+2} \prec \omega_{F+2}^{(h)}}} \cdots \sum_{\sup _{L}^{\left(h^{\prime}\right)} \prec \omega_{L}^{(h)}} \cdot \sum_{\substack{\omega_{i}^{\left(h^{\prime}\right)} \in O_{h^{\prime}} \\ i \neq F+1}}\left[\sum_{\substack{\omega_{F+1}^{\left(h^{\prime}\right)} \prec \omega_{F+1}^{(h)} \\ \omega_{L}}} \chi\left(\Omega_{L}^{h^{\prime}}\right)\right] .
$$

Now, the last sum can be bounded as $\sum_{\omega_{1}^{\left(h^{\prime}\right)} \prec \omega_{1}^{(h)}} \chi\left(\Omega_{L}^{h^{\prime}}\right) \leq c^{L}$, simply by momentum conservation. After this bound, we see that the remaining sums contribute with a factor $\gamma^{\frac{1}{2}\left(h-h^{\prime}\right)(L-F-1)}$ and the lemma is proved.

\section{D.1. The case $F=3$}

We start with the analysis of the case $F=3$. We assume that the number of endpoints $n$ of the tree is $n \geq 2$, the case $n=1$ being already treated, see (D.1).

As in Sect. 2.8, we denote by $\tilde{v}_{0}$ the first $c$-vertex following the root and we call $h_{0}$ its scale. Then, we bound the product of $\chi$-functions in (2.117) as in (2.107) and, using the latter estimate, we bound (2.117) by the r.h.s. of (2.108), where the $*$ on the sums must be interpreted as meaning that all the sectors index in $\Omega_{\text {ext }}^{(3)}$ must not be summed over. With this interpretation of the $*$ on the sum and using Lemma D.1 for $4 \leq L \leq 8$ and the trivial bound $\gamma^{\left(h_{0}-h\right)(L-3) / 2}$ for $L \geq 10$, 
we perform the bound analogue to (2.109):

$$
\sum_{\Omega_{\tilde{v}_{0}}^{(h)} \prec \Omega_{\tilde{v}_{0}}^{\left(h_{0}\right)}}^{*} \chi\left(\Omega_{\tilde{v}_{0}}^{(h)}\right) \leq c \gamma^{\frac{1}{2}\left(h_{0}-h\right)\left[\left|P_{\tilde{v}_{0}}\right|-4+\mathbb{1}\left(\left|P_{\tilde{v}_{0}}\right|>10\right)\right],}
$$

and we see that, with respect to (2.109), we gain at least a factor $\gamma^{\frac{1}{2}\left(h-h_{0}\right)}$. Now, proceeding through the analogues of (2.110) and (2.111), we rewrite the left over expression in the same form as the r.h.s. of (2.111):

$$
\sum_{\Omega_{\tilde{\underline{v}}_{0}}^{\left(h_{0}\right)} \backslash \Omega_{\mathrm{ext}}^{(3)}} \prod_{v \in \underline{\tilde{v}}_{0}} F_{v}\left(\Omega_{v}^{\left(h_{0}\right)}\right) \prod_{l \in T_{\tilde{v}_{0}}} \delta_{\omega_{l}^{+}, \omega_{l}^{-}} .
$$

We now distinguish three possible cases.

(1) If the lines with field labels $f_{1}, f_{2}, f_{3}$ are all incident to the same vertex $v_{\mathbf{x}} \in \underline{\tilde{v}}_{0}$, we choose $v_{\mathbf{x}}$ as the root of $T_{\tilde{\underline{v}}_{0}}$ and, "pruning $T_{\tilde{\underline{v}}_{0}}$ of its leaves", one after the other, via the same procedure described after (2.111), we can bound (D.5) by the analogue of (2.115) that takes the form:

$$
\sup _{\Omega_{\mathrm{ext}}^{(3)}} \sum_{\Omega_{v_{\mathbf{x}}}^{\left(h_{0}\right)} \backslash \Omega_{\text {ext }}^{(3)}} F_{v_{\mathbf{x}}}\left(\Omega_{v_{\mathbf{x}}}^{\left(h_{0}\right)}\right) \prod_{v \in \underline{\tilde{v}}_{0} \backslash v_{\mathbf{x}}} \sup _{\omega_{v}^{*}} \sum_{\Omega_{v}^{\left(h_{0}\right)} \backslash \omega_{v}^{*}} F_{v}\left(\Omega_{v}^{\left(h_{0}\right)}\right)
$$

where $\omega_{v}^{*}$ is defined as after (2.115). Now, each factor in the product over $v \in \underline{\tilde{v}}_{0} \backslash v_{\mathbf{x}}$ can be bounded exactly as explained after (2.115), that is as in (2.116) if $v$ is an endpoint or, if it is not, by the r.h.s. of $(2.109)$, with $\left(h_{0}-h\right)$ replaced by $\left(h_{v}-h_{0}\right)$ and $\left|P_{\tilde{v}_{0}}\right|$ replaced by $\left|P_{v}\right|$. As regarding the first factor in (D.6), if $v_{\mathbf{x}}$ is an endpoint, it can be bounded by constant, that is it gives a contribution $\gamma^{h_{0} / 2}$ smaller than the corresponding (2.116); in this case, combining this gain with the gain $\gamma^{\frac{1}{2}\left(h-h_{0}\right)}$ found above, see (D.4) and the following comment, the desired dimensional gain is found. If $v_{\mathbf{x}}$ is not an endpoint, we are still left with $\sup _{\Omega_{\text {ext }}^{(3)}} \sum_{\Omega_{v_{\mathbf{x}}}^{\left(h_{0}\right)} \backslash \Omega_{\text {ext }}^{(3)}} F_{v_{\mathbf{x}}}\left(\Omega_{v_{\mathbf{x}}}^{\left(h_{0}\right)}\right)$ and we still have to establish whether this factor admits a dimensional gain with respect to the corresponding factor in (2.115). We shall discuss this below, after item (3).

(2) If two of the lines with field labels in $\left\{f_{1}, f_{2}, f_{3}\right\}$, say $f_{1}, f_{2}$, are incident to the same vertex $v_{\mathbf{x}}$, while $f_{3}$ is incident to a distinct vertex $v_{\mathbf{y}} \in \underline{\tilde{v}}_{0}$, we identify the path $l_{\mathbf{x}, \mathbf{y}}$ on $T_{\tilde{v}_{0}}$ connecting $v_{\mathbf{x}}$ and $v_{\mathbf{y}}$, we denote by $T_{\mathbf{x}}$ the subtree of $T_{\tilde{v}_{0}}$ rooted on $v_{\mathbf{x}}$ and with no lines in common with $l_{\mathbf{x}, \mathbf{y}}$ and by $\underline{\tilde{v}}_{0}^{\mathbf{x}} \subset \underline{\tilde{v}}_{0}$ the set of vertices in $T_{\mathbf{x}}$ (note that possibly $T_{\mathbf{x}}$ is trivial, that is $\underline{\tilde{v}}_{0}^{\mathbf{x}}=\left\{v_{\mathbf{x}}\right\}$ ). In analogy with (2.112), we define

$$
\begin{aligned}
& \mathcal{F}_{\underline{\underline{v}}_{0} \backslash \underline{\tilde{v}}_{0}^{\times}}\left(\Omega_{\underline{\underline{v}}_{0} \backslash \underline{\underline{v}}_{0}^{\times}}^{\left(h_{0}\right)} \stackrel{\text { def }}{=} \prod_{v \in \underline{\underline{v}}_{0} \backslash \underline{\underline{\tilde{v}}}_{0}^{\times}} F_{v}\left(\Omega_{v}^{\left(h_{0}\right)}\right) \prod_{l \in T_{\tilde{v}_{0}} \backslash\left\{T_{\mathbf{x}} \cup l_{\mathbf{x}}^{*}\right\}} \delta_{\omega_{l}^{+}, \omega_{l}^{-}},\right. \\
& \mathcal{F}_{\underline{\tilde{v}}_{0}^{\times}}\left(\Omega_{\underline{\underline{v}}_{0}^{\times}}^{\left(h_{0}\right)}\right) \stackrel{\text { def }}{=} \prod_{v \in \underline{\tilde{v}}_{0}^{\times}} F_{v}\left(\Omega_{v}^{\left(h_{0}\right)}\right) \prod_{l \in T_{\mathbf{x}}} \delta_{\omega_{l}^{+}, \omega_{l}^{-}},
\end{aligned}
$$


where $l_{\mathbf{x}}^{*}$ denotes the line of $T_{\tilde{v}_{0}} \backslash T_{\mathbf{x}}$ incident to $v_{\mathbf{x}}\left(f_{\mathbf{x}}^{*}\right.$ and $\omega_{\mathbf{x}}^{*}$ will be its field and sector indices); we can rewrite (D.5) as

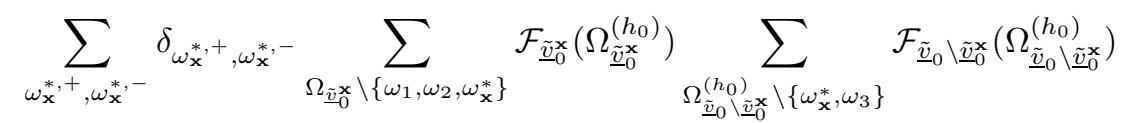

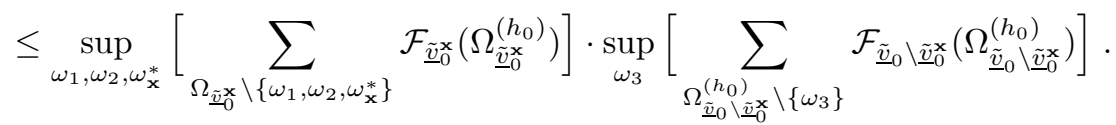

Now, the second factor in (D.8) can be bounded following the same procedure described in Section 2.8 and we do not find neither a gain nor a loss with respect to the estimates in Section 2.8. The first factor can be studied as in item (1) above and, if $v_{\mathbf{x}}$ is an endpoint, we gain $\gamma^{h_{0} / 2}$ with respect to the estimates in Section 2.8; in this case, combining this gain with the gain $\gamma^{\frac{1}{2}\left(h-h_{0}\right)}$ found above, the desired dimensional gain is found. If $v_{\mathbf{x}}$ is not an endpoint, we are still left with $\sup _{\Omega_{\text {ext }}^{(3)}} \sum_{\Omega_{v_{\mathbf{x}}}^{\left(h_{0}\right)} \backslash \Omega_{\text {ext }}^{(3)}} F_{v_{\mathbf{x}}}\left(\Omega_{v_{\mathbf{x}}}^{\left(h_{0}\right)}\right)$ and we still have to establish whether this factor admits a dimensional gain with respect to the corresponding factor in (2.115). We shall discuss this below, after item (3).

(3) If the lines with field labels $f_{1}, f_{2}, f_{3}$ are all incident to different vertices in $\underline{\underline{v}}_{0}$, call them $v_{\mathbf{x}}, v_{\mathbf{y}}, v_{\mathbf{z}}$, we again identify the path $l_{\mathbf{x}, \mathbf{y}}$ on $T_{\tilde{v}_{0}}$ connecting $v_{\mathbf{x}}$ and $v_{\mathbf{y}}$ and we define $T_{\mathbf{x}}$ and $\underline{\tilde{v}}_{0}^{\mathbf{x}}$ as in item (2). With no loss of generality we can assume that $v_{\mathbf{z}} \in \underline{\tilde{v}}_{0}^{\mathbf{X}}$ : it is in fact easy to realize that, if $v_{\mathbf{z}} \notin \underline{\tilde{v}}_{0}^{\mathbf{x}}$, we can always permute the symbols $v_{\mathbf{x}}, v_{\mathbf{y}}, v_{\mathbf{z}}$ in such a way that, after the permutation, $v_{\mathbf{z}} \in \underline{\tilde{v}}_{0}^{\mathbf{x}}$. Repeating the discussion in item (2) we can again bound the expression under analysis by the r.h.s. of (D.8), in which now the first factor has the same structure as the term studied in item (2) (it corresponds to a sector sum in which two of the lines corresponding to external fixed sectors are incident to $v_{\mathbf{x}}$, while the third one is incident to a different vertex, $v_{\mathbf{z}}$ ). So, by the proof in item (2), we find that, if $v_{\mathbf{x}}$ is an endpoint, we soon find the desired dimensional gain, otherwise we are still left with $\sup _{\Omega_{\mathrm{ext}}^{(3)}} \sum_{\Omega_{v_{\mathbf{x}}}^{\left(h_{0}\right)} \backslash \Omega_{\mathrm{ext}}^{(3)}} F_{v_{\mathbf{x}}}\left(\Omega_{v_{\mathbf{x}}}^{\left(h_{0}\right)}\right)$ and we still have to establish whether this factor admits a dimensional gain with respect to the corresponding factor in (2.115).

In all cases discussed above, either $v_{\mathbf{x}}$ is an endpoint, in which case the desired dimensional bound is found, or it is not, and we are left with an expression completely analogous to the initial one, with the external scale $h$ replaced by $h_{0}$. Then it is clear that we can iteratively enter the structure of $v_{\mathbf{x}}$ following the same procedure described in items (1)-(3) above and, proceeding by induction, we find the desired gain.

\section{D.2. The case $F=5$}

Let us now consider $F=5$. Using the same notations as in Section D.1 and following the same procedure, we bound the product of $\chi$-functions in (2.117) as in (2.107) and, using the latter estimate, we bound (2.117) by the r.h.s. of (2.108), 
where the $*$ on the sums must be interpreted as meaning that all the sectors index in $\Omega_{\text {ext }}^{(5)}$ must not be summed over. With this interpretation of the $*$ on the sum, we perform the bound analogue to (2.109) and (D.4):

$$
\sum_{\Omega_{\tilde{v}_{0}}^{(h)} \prec \Omega_{\tilde{v}_{0}}^{\left(h_{0}\right)}}^{*} \chi\left(\Omega_{\tilde{v}_{0}}^{(h)}\right) \leq c \gamma^{\frac{1}{2}\left(h_{0}-h\right)\left[\left|P_{\tilde{v}_{0}}\right|-6+\mathbb{1}\left(\left|P_{\tilde{v}_{0}}\right|>10\right)\right]},
$$

and we see that, with respect to (2.109), we gain at least a factor $\gamma^{\left(h-h_{0}\right)}$. We now rewrite the left over expression in a form similar to (D.5):

$$
\sum_{\Omega_{\underline{\underline{v}}_{0}}^{\left(h_{0}\right)} \backslash \Omega_{\mathrm{ext}}^{(5)}} \prod_{v \in \underline{\tilde{v}}_{0}} F_{v}\left(\Omega_{v}^{\left(h_{0}\right)}\right) \prod_{l \in T_{\tilde{v}_{0}}} \delta_{\omega_{l}^{+}, \omega_{l}^{-}} .
$$

We now distinguish three possible cases.

(1) If the lines with field labels $f_{1}, f_{2}, f_{3}, f_{4}, f_{5}$ are all incident to the same vertex $v_{\mathbf{x}} \in \underline{\tilde{v}}_{0}$, we choose $v_{\mathbf{x}}$ as the root of $T_{\underline{\tilde{v}}_{0}}$ and, proceeding as in item (1) of Section D.1, we are left with the analogue of (D.6):

$$
\sup _{\Omega_{\text {ext }}^{(5)}} \sum_{\Omega_{v_{\mathbf{x}}}^{\left(h_{0}\right)} \backslash \Omega_{\text {ext }}^{(5)}} F_{v_{\mathbf{x}}}\left(\Omega_{v_{\mathbf{x}}}^{\left(h_{0}\right)}\right) \prod_{v \in \tilde{\underline{v}}_{0} \backslash v_{\mathbf{x}}} \sup _{\omega_{v}^{*}} \sum_{\Omega_{v}^{\left(h_{0}\right)} \backslash \omega_{v}^{*}} F_{v}\left(\Omega_{v}^{\left(h_{0}\right)}\right)
$$

As discussed in item (1) of Section D.1, each factor in the product over $v \in \underline{\tilde{v}}_{0} \backslash v_{\mathbf{x}}$ is not associated to any gain or loss (it can be bounded as in Section 2.8). Note that now $v_{\mathbf{x}}$ cannot be an endpoint (it has at least 6 external lines), so we are still left with $\sup _{\Omega_{\text {ext }}^{(5)}} \sum_{\Omega_{v_{\mathbf{x}}}^{\left(h_{0}\right)} \backslash \Omega_{\text {ext }}^{(3)}} F_{v_{\mathbf{x}}}\left(\Omega_{v_{\mathbf{x}}}^{\left(h_{0}\right)}\right)$ and we still have to establish whether this factor admits a dimensional gain with respect to the corresponding factor in (2.115). We shall discuss this below, after item (3).

(2) Calling $v_{\mathbf{x}_{1}}, \ldots, v_{\mathbf{x}_{5}}$ the vertices to which the lines $l_{1}, \ldots, l_{5}$ labelled by $f_{1}, \ldots f_{5}$ are incident to, if $v_{\mathbf{x}_{1}}, \ldots, v_{\mathbf{x}_{5}}$ are not all coinciding, we can assume without loss of generality that $v_{\mathbf{x}_{1}} \neq v_{\mathbf{x}_{5}}$ and we can consider the path $l_{\mathbf{x}_{1}, \mathbf{x}_{5}}$ on $T_{\tilde{v}_{0}}$ connecting $v_{\mathbf{x}_{1}}$ and $v_{\mathbf{x}_{5}}$. Given $l^{*} \in l_{\mathbf{x}_{1}, \mathbf{x}_{5}}$, we call $T_{1}$ and $T_{2}$ the two disjoint subtrees of $T_{\tilde{v}_{0}}$ obtained by disconnecting from $T_{\tilde{v}_{0}}$ the line $l^{*}$ : we assume that $T_{1}$ is anchored to $v_{\mathbf{x}_{1}}$ and $T_{2}$ is anchored to $v_{\mathbf{x}_{5}}$. We call $\underline{\tilde{v}}_{0}^{1}, \underline{\tilde{v}}_{0}^{2}$ the two disjoint subsets of $\underline{\tilde{v}}_{0}$ connected by the lines of $T_{1}, T_{2}$ respectively.

We now further distinguish two more subcases.

(2.a) If there is a way of choosing $l^{*} \in l_{\mathbf{x}_{1}, \mathbf{x}_{5}}$ so that three among the lines in $\left\{l_{1}, \ldots, l_{5}\right\}$ are incident to $T_{1}$ and two of them are incident to $T_{2}$ (or vice versa), we can assume without loss of generality that $f_{1}, f_{2}, f_{3}$ are incident to $T_{1}$ and $f_{4}, f_{5}$ are incident to $T_{2}$. In this case, in analogy with the definition (D.7), we define

$$
\begin{aligned}
& \mathcal{F}_{\underline{\underline{v}}_{0}^{1}}\left(\Omega_{\underline{\underline{v}}_{0}^{1}}^{\left(h_{0}\right)}\right) \stackrel{\text { def }}{=} \prod_{v \in \underline{\tilde{v}}_{0}^{1}} F_{v}\left(\Omega_{v}^{\left(h_{0}\right)}\right) \prod_{l \in T_{1}} \delta_{\omega_{l}^{+}, \omega_{l}^{-}}, \\
& \mathcal{F}_{\underline{\underline{v}}_{0}^{2}}\left(\Omega_{\underline{\underline{v}}_{0}^{2}}^{\left(h_{0}\right)}\right) \stackrel{\text { def }}{=} \prod_{v \in \underline{\tilde{v}}_{0}^{2}} F_{v}\left(\Omega_{v}^{\left(h_{0}\right)}\right) \prod_{l \in T_{2}} \delta_{\omega_{l}^{+}, \omega_{l}^{-}},
\end{aligned}
$$


and, calling $\omega_{*}$ the sector index of $l^{*}$, we rewrite (D.10) as:

$$
\begin{aligned}
& \sum_{\omega_{*}^{+}, \omega_{*}^{-}} \delta_{\omega_{*}^{+}, \omega_{*}^{-}} \sum_{\Omega_{\underline{\underline{v}}_{0}^{1}} \backslash\left\{\omega_{1}, \omega_{2}, \omega_{3}, \omega_{*}\right\}} \mathcal{F}_{\underline{\underline{v}}_{0}^{1}}\left(\Omega_{\underline{\underline{v}}_{0}^{1}}^{\left(h_{0}\right)}\right) \sum_{\Omega_{\Omega_{\underline{v}_{0}^{2}}^{(h)} \backslash\left\{\omega_{*}, \omega_{4}, \omega_{5}\right\}}} \mathcal{F}_{\underline{\underline{v}}_{0}^{2}}\left(\Omega_{\underline{\underline{v}}_{0}^{2}}^{\left(h_{0}\right)}\right) \\
& \leq \sup _{\omega_{1}, \omega_{2}, \omega_{3},}\left[\sum_{\Omega_{\underline{\underline{v}}_{0}^{1}} \backslash\left\{\omega_{1}, \omega_{2}, \omega_{3}\right\}} \mathcal{F}_{\underline{\underline{v}}_{0}^{1}}\left(\Omega_{\underline{\underline{v}}_{0}^{1}}^{\left(h_{0}\right)}\right)\right] \cdot \sup _{\omega_{*}, \omega_{4}, \omega_{5}}\left[\sum_{\Omega_{\underline{\underline{v}}_{0}^{2}}^{\left(h_{0}\right)} \backslash\left\{\omega_{*}, \omega_{4}, \omega_{5}\right\}} \mathcal{F}_{\underline{\underline{v}}_{0}^{2}}\left(\Omega_{\underline{\underline{v}}_{0}^{2}}^{\left(h_{0}\right)}\right)\right] .
\end{aligned}
$$

Now both factors can be bounded as in Section D.1 and we get a gain $\gamma^{h_{0} / 2}$ from both, and in this case the desired dimensional gain is found.

(2.b) If any choice of $l^{*} \in l_{\mathbf{x}_{1}, \mathbf{x}_{5}}$ is such that four among the lines in $\left\{l_{1}, \ldots, l_{5}\right\}$ are incident to $T_{1}$ and only one of them is incident to $T_{2}$ (or vice versa), we can assume without loss of generality that $f_{1}, f_{2}, f_{3}, f_{4}$ are incident to $T_{1}$ and $f_{5}$ is incident to $T_{2}$. In this case, with the same definitions (D.12) and calling $\omega_{*}$ the sector index of $l^{*}$, we can rewrite (D.10) as:

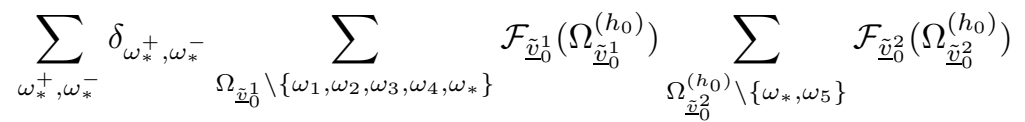

$$
\begin{aligned}
& \leq \sup _{\omega_{1}, \omega_{2}, \omega_{3}, \omega_{4}, \omega_{*}}\left[\sum_{\Omega_{\tilde{v}_{0}^{1}} \backslash\left\{\omega_{1}, \omega_{2}, \omega_{3}, \omega_{4}, \omega_{*}\right\}} \mathcal{F}_{\underline{\underline{v}}_{0}^{1}}\left(\Omega_{\tilde{\underline{v}}_{0}^{1}}^{\left(h_{0}\right)}\right)\right] \cdot \sup _{\omega_{5}}\left[\sum_{\Omega_{\underline{\underline{v}}_{0}^{2}}^{\left(h_{0}\right)} \backslash\left\{\omega_{5}\right\}} \mathcal{F}_{\underline{\tilde{v}}_{0}^{2}}\left(\Omega_{\underline{\tilde{v}}_{0}^{2}}^{\left(h_{0}\right)}\right)\right] .
\end{aligned}
$$

Now the second factor can be bounded exactly as in Section 2.8 and then it is not associated to any gain or loss. The first factor has again the same structure as (D.10) and we can again bound it following the same procedure described in items (1), (2.a) and (2.b) above. Following iteratively the procedure, either we get the desired dimensional gain (if we recover the case of (2.a) above) or we are left with an expression analogue to (D.11).

But the first factor in (D.11) has exactly the same structure as (D.10), with th external scale $h$ replaced by $h_{0}$. Then it is clear that we can iteratively enter the structure of $v_{\mathbf{x}}$ following the same procedure described in items (1)-(2.b) above and, proceeding by induction, we find the desired gain. This concludes the proof of Lemma 2.6.

\section{Acknowledgments}

Part of this work was done in Vienna at the Erwin Schrödinger International Institute for Mathematical Physics during the program "Many body quantum theory". We thank the organizers M. Salmhofer and J. Yngvason for their nice invitation. AG was partially supported by a Junior Research Fellowship from ESI, by M.I.U.R. and by U.S. National Science Foundation grant PHY 01 39984, which are gratefully acknowledged. AG wants also to thank M. Salmhofer for some enlightening discussions. 


\section{References}

[A] P.W. Anderson, The theory of superconductivity on high $T_{c}$ cuprates, Princeton University Press, Princeton (1997).

[AMR] S. Afchain, J. Magnen, V. Rivasseau, Ann. Henri Poincaré 6 (2005), 399-448 and 449-483.

[BGN] G. Benfatto, A. Giuliani, V. Mastropietro, Ann. Henri Poincaré 4 (2003), 137193.

[BM] G. Benfatto, V. Mastropietro, Rev. Math. Phys. 13 (2001), 1323-143.

[DR] G. Disertori, V. Rivasseau, Comm. Math. Phys. 215 (2000), 291-341 and 251390 .

[FKT] J. Feldman, H. Knoerrer, E. Trubowitz, Comm. Math. Phys. 247 (2004), 1-320.

[FMRT] J. Feldman, J. Magnen, V. Rivasseau, E. Trubowitz, Helv. Phys. Acta 65 (1992), 679-721.

[FST] J. Feldman, M. Salmhofer, E. Trubowitz, J. Statist. Phys. 84 (1996), 1209-1336; Comm. Pure Appl. Math. 51 (1998), 1133-1246; Comm. Pure Appl. Math. 52 (1999), 273-324; Comm. Pure Appl. Math. 53 (2000), 1350-1384.

[G] P. Goldbaum, Comm. Math. Phys. 258 (2005), 317-337.

[GLM] G. Gallavotti, J.L. Lebowitz, V. Mastropietro, J. Statist. Phys. 108 (2002), 831-861.

[GM] G. Gentile, V. Mastropietro, Phys. Rep. 352 (2001), 273-43.

[GS] V.M. Galitski, S. Das Sarma, Phys. Rev. B 70 (2004), 035111.

[HM] C.J. Halboth, W. Metzner, Z. Phys. B 102 (1997), 501-504.

[KT] T. Koma, H. Tasaki, Phys. Rev. Lett. 68 (1992), 3248-3252.

[L] E.H. Lieb, in The Hubbard model; its physics and mathematical physics, Edited by D. Baeriswyl, D. Campbell et al, Nato ASI series 343 (1995), 1-19.

[L1] E.H. Lieb, Phys. Rev. Lett. 62 (1989), 1201-1205.

[LW] E.H. Lieb, F.Y. Wu, Phys. Rev. Lett. 20 (1968), 1445-1449.

[Le] A. Lesniewski, Comm. Math. Phys. 108 (1987), 437-467.

[M] V. Mastropietro, J. Stat. Phys. 121 (2005), 373-432.

[Me] W. Metzner, Int. J. Modern Phys. A 16 (2001), 1889-1898.

[NO] J.W. Negele, H. Orland, Quantum Many-Particle Systems, Addison Wesley, New York (1998).

[PS] S. Pedra, M. Salmhofer, Proceeding of ICMP 2003.

[R] V. Rivasseau, J. Stat. Phys. 106 (2002), 693-722.

[S] J. Solyom, Adv. Phys. 28 (1979), 201-303.

[Sa1] M. Salmhofer, Comm. Math. Phys. 194 (1998), 249-295.

[Sa2] M. Salmhofer, Rev. Math. Phys. 10 (1998), 553-578.

[VR] A. Virosztek, J. Ruvalds, Phys. Rev. B 42 (1990), 4064-4072. 
G. Benfatto and V. Mastropietro Mathematics Department University of Rome "Tor Vergata" Via della Ricerca Scientifica I-00133 Roma

Italy

Supported by M.I.U.R.

e-mail: benfatto@mat.uniroma2.it mastropi@mat . uniroma2. it

A. Giuliani

Physics Department

Princeton University

Jadwin Hall

Princeton, NJ, 08544

USA

e-mail: giuliani@princeton.edu

Communicated by Vincent Rivasseau

Submitted: January 4, 2006

Accepted: January 31, 2006

(D) To access this journal online:

(4P) http://www.birkhauser.ch 